\title{
Steam Reforming of Low-Level Mixed Waste
}

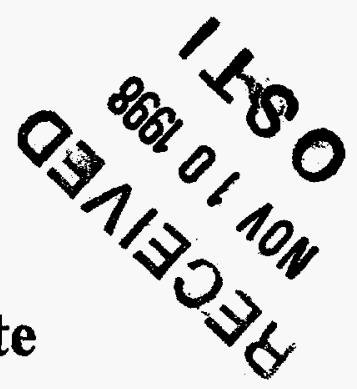

\author{
Topical Report \\ January 1998
}

Work Performed Under Contract No.: DE-AR21-95MC32091

\author{
For \\ U.S. Department of Energy \\ Office of Fossil Energy \\ Federal Energy Technology Center \\ P.O. Box 880 \\ Morgantown, West Virginia 26507-0880
}

ThermoChem, Inc.

10220-H Old Columbia Road

Columbia, Maryland 21046 


\section{Disclaimer}

This report was prepared as an account of work sponsored by an agency of the United States Government. Neither the United States Government nor any agency thereof, nor any of their employees, makes any warranty, express or implied, or assumes any legal liability or responsibility for the accuracy, completeness, or usefulness of any information, apparatus, product, or process disclosed, or represents that its use would not infringe privately owed rights. Reference herein to any specific commercial product, process, or service by trade name, trademark, manufacturer, or otherwise does not necessarily constitute or imply its endorsement, recommendation, or favoring by the United States Government or any agency thereof. The views and opinions of authors expressed herein do not necessarily state or reflect those of the United States Government or any agency thereof. 


\section{DISCLAIMER}

Portions of this document may be illegible in electronic image products. Images are produced from the best available original document. 


\section{TABLE OF CONTENTS}

Page

ABSTRACT

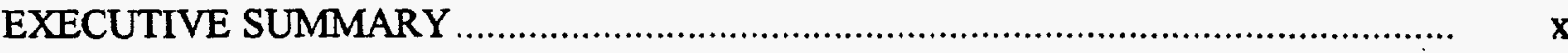

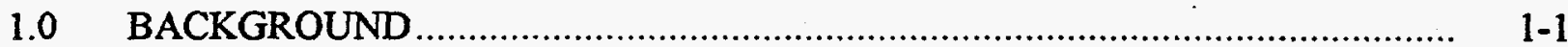

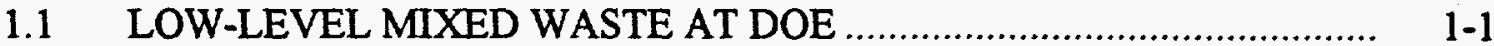

1.2 THERMOCHEM STEAM REFORMING ........................................ $1-2$

1.2.1 Technology Description ................................................... 1-2

1.2.2 Applicability of ThermoChem Steam Reforming to LLMW Treatment............................................................ 1-9

1.2.3 Development Status ................................................... $\quad 1-12$

2.0 PROGRAM OBJECTIVES AND SCOPE ..............................................

$2.1 \quad$ PROGRAM OBJECTIVES .......................................................

2.2 WORK TASKS ...................................................................... $2-2$

3.0 SURROGATE IDENTIFICATION AND PREPARATION ............................

4.0 PROCESS DEVELOPMENT UNIT (PDU) DESIGN AND CONSTRUCTION

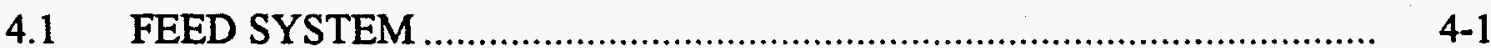

4.1.1 Liquid Feed System................................................................... 4-1

4.1.2 Original Solid Feed System .................................................. 4-1

4.1.3 New Solid Feed System (Piketon Test) .................................... . 4-4

4.1.4 Piketon Feedstock Shredding System ........................................ 4-4

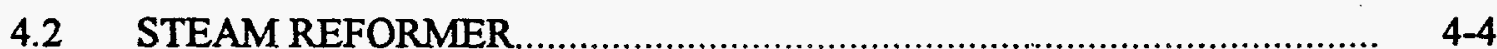

4.3 STEAM REFORMER GAS CLEANUP ..........................................

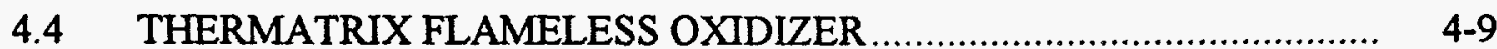

4.5 THERMATRIX GAS CLEANUP ................................................ $4-12$ 


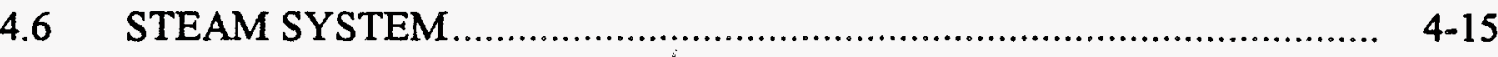

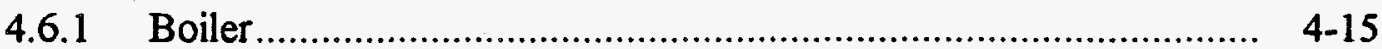

4.6.2 Superheater ...................................................................... 4-15

$5.0 \quad$ TEST PLAN

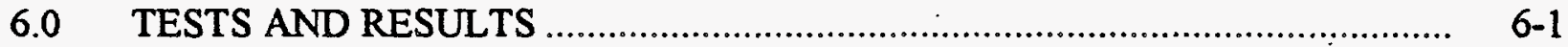

$6.1 \quad$ TEST OBJECTIVES ...................................................................... 6-1

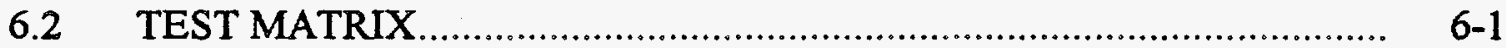

$6.3 \quad$ PRELIMINARY SCREENING TEST …............................................

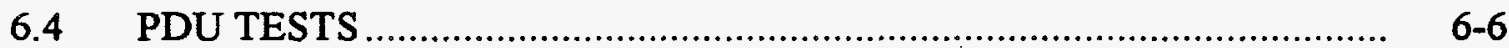

6.4.1 Test Description........................................................................... 6

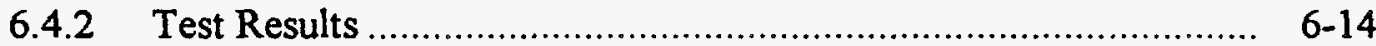

6.4.3 Discussion of Results ................................................... 6.58

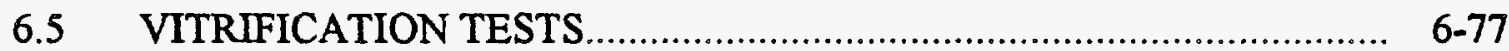

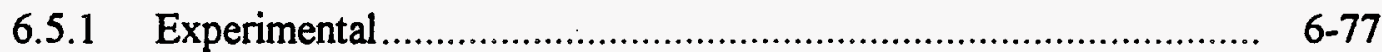

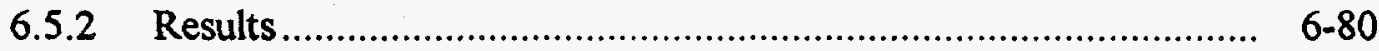

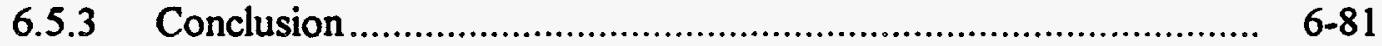

7.0 PRELIMINARY CONCEPTUAL DESIGN …..............................................

$7.1 \quad$ PROCESS FLOW OVERVIEW …………......................................

7.1.1 Conceptual Facility............................................................... 7-1

7.1.2 General Arrangement .......................................................... 7-8

7.2 POTENTIAL SITES

7.2.1 Sites Considered ………….................................................... 7-10

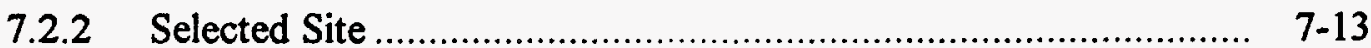




\section{TABLE OF CONTENTS}

(Continued)

Page

8.0 ECONOMIC EVALUATION/STEAM REFORMING ....................................

8.1 STEAM REFORMER CAPITAL AND OPERATING COST ESTIMATE ................................................................... 8-1

8.1.1 Capital Costs......................................................................... 8-1

8.1.2 Steam Reformer Economic Summary ........................................ 8-3

8.2 COMPETING SYSTEMS ECONOMICS .......................................... 8-7

8.3 DEMONSTRATION/COMMERCIAL UNIT COMPARISON ................... 8-9

\section{REFERENCES}

ACRONYMS

APPENDIX A: SURROGATE FEEDSTOCK IDENTIFICATION

AND PREPARATION

APPENDIX B: TASK 1.2.1: REVISED TEST PLAN

APPENDIX C: LETTERS OF SUPPORT

APPENDIX D: OPERATING PARAMETER TRENDS

- Multiple Surrogate Tests, and

- Piketon Waste Simulation Tests 


\section{LIST OF FIGURES}

Page

FIGURE 1-1: PROCESS DEVELOPMENT UNIT (PDU)

FLOW DIAGRAM

FIGURE 4-1: LIQUID FEED SYSTEM …............................................................

FIGURE 4-2: ORIGINAL SOLID FEED SYSTEM ………………..........................

FIGURE 4-3: NEW FEED HOPPER SYSTEM .....................................................

FIGURE 4-4: ～LOCK HOPPER AND INJECTION SCREW ......................................... $\quad 4-6$

FIGURE 4-5: $\quad$ PIKETON FEEDSTOCK SHREDDING SYSTEM …............................ $\quad$ 4-7

FIGURE 4-6: $\quad$ STEAM REFORMER

FIGURE 4-7 ELECTRIC HEATER MODULES .................................................. 4-10

FIGURE 4-8 HEPA FILTERS ................................................................... 4-11

FIGURE 4-9 FLAMELESS OXIDIZER …........................................................ 4-13

FIGURE 4-10 FLAMELESS OXIDIZER BoP..................................................... 4-14

FIGURE 4-11: SATURATED STEAM BOILER AND FEED

WATER SYSTEM ............................................................... $4-16$

FIGURE 4-12: STEAM SUPERHEATERS …...................................................... 4-17

FIGURE 6-1: SCHEMATIC OF APPARATUS USED FOR

SCREENING TEST …..................................................................

FIGURE 6-2: STEAM REFORMER FLUIDIZATION FLOW …............................... $\quad$ 6-28

FIGURE 6-3: STEAM REFORMER FREEBOARD PRESSURE …............................ $\quad 6-29$

FIGURE 6-4: HIGH-TEMPERATURE HEPA FILTER PRESSURE DROP …............ $\quad 6-30$ 


\section{LIST OF FIGURES}

(Continued)

FIGURE 6-5: TEMPERATURE PROFILES.....................................................

FIGURE 6-6: HEAT INPUT TO STEAM REFORMER ......................................... $\quad 6-32$

FIGURE 6-7: STEAM REFORMER FLUIDIZATION FLOW .................................... $\quad 6-47$

FIGURE 6-8: $\quad$ STEAM REFORMER FREEBOARD PRESSURE ............................... $\quad 6-48$

FIGURE 6-9: HIGH-TEMPERATURE HEPA FILTER PRESSURE DROP ................ $\quad 6-49$

FIGURE 6-10: TEMPERATURE PROFILES...................................................... 6-50

FIGURE 6-11: HEAT INPUT TO STEAM REFORMER .......................................... 6-51

FIGURE 6-12: CERIUM LOADING AND PARTITIONING ......................................

FIGURE 7-1: PROCESS FLOW DIAGRAM - CASE 1, RESINS ………….............. $\quad$ 7-2

FIGURE 7-2: PROCESS FLOW DIAGRAM - CASE 2, ABSORBENT PADS ............ 7-3

FIGURE 7-3: $\quad$ STEAM REFORMER ……............................................................. $\quad$ 7-6

FIGURE 7-4: CONCEPTUAL FACILITY - PLAN VIEW …....................................... 


\section{LIST OF TABLES}

Page

TABLE 1-1: THERMATRIX ${ }^{\circledR}$ FLAMELESS THERMAL OXIDATION SYSTEMS - INSTALLATION LIST …............................................. 1-14

TABLE 3-1: SURROGATE FORMULATION - SUMMARY ……….......................

TABLE 3-2: FEEDSTOCK 7 (Represents Piketon Waste) ........................................... $\quad 3-4$

TABLE 3-3: ULTIMATE ANALYSIS OF THE SURROGATE FEEDSTOCKS ........... 3-5

TABLE 6-1: ANALYSIS OF RESIN ....................................................................

TABLE 6-2: $\quad$ MULTIPLE ORGANIC FEEDSTOCK RETESTS …................................

TABLE 6-3: MULTIPLE SURROGATE TEST RESULTS SUMMARY OPERATING CONDITIONS ....................................................... $\quad 6-10$

TABLE 6-4: PIKETON WASTE SIMULATION TEST RESULTS SUMMARY - OPERATING CONDITIONS

TABLE 6-5: MULTIPLE ORGANIC FEEDSTOCK RETEST RESULTS SUMMARY - OPERATING CONDITIONS.

TABLE 6-6: MULTIPLE SURROGATE TEST RESULTS SUMMARY DESTRUCTION AND REMOVAL EFFICIENCY (DRE)

TABLE 6-7: MULTIPLE SURROGATE TEST RESULTS SUMMARY SURROGATE RADIONUCLIDES/METALS RETENTION

TABLE 6-8: MULTIPLE SURROGATE TEST RESULTS SUMMARY CONVERSION, CAPTURE AND CLOSURE

TABLE 6-9: MULTIPLE SURROGATE TEST RESULTS SUMMARY HIGH-TEMPERATURE HEPA EXIT GAS COMPOSITION

TABLE 6-10: MULTIPLE SURROGATE TEST RESULTS SUMMARY STACK FLUE GAS COMPOSITION

TABLE 6-11: SUMMARY OF PCDD EMISSION RATES FEEDSTOCK NO. 6 


\section{LIST OF TABLES}

(Continued)

TABLE 6-12: SUMMARY OF PCDF EMISSION RATES FEEDSTOCK NO. 6.

TABLE 6-13: SUMMARY OF PCDD EMISSION RATES FEEDSTOCK NO. 2

TABLE 6-14: SUMMARY OF PCDF EMISSION RATES FEEDSTOCK NO. 2

TABLE 6-15: PIKETON WASTE SIMULATION TEST RESULTS SUMMARY - DESTRUCTION AND REMOVAL EFFICIENCY

TABLE 6-16: PIKETON WASTE SIMULATION TEST RESULTS SUMMARY - SURROGATE AND RADIONUCLIDE RETENTION 6-34

TABLE 6-17: PIKETON WASTE SIMULATION TEST RESULTS SUMMARY - CONVERSION, CAPTURE AND CLOSURE 6-35

TABLE 6-18: PIKETON WASTE SIMULATION TEST RESULTS SUMMARY - HIGH-TEMPERATURE HEPA EXIT GAS COMPOSITION

TABLE 6-19: PIKETON WASTE SIMULATION TEST RESULTS SUMMARY - STACK FLUE GAS COMPOSITION

TABLE 6-20: SUMMARY OF PCDD EMISSION RATES FOR PIKETON WASTE TESTS 1 AND 2 6-38

TABLE 6-21: SUMMARY OF PCDF EMISSION RATES FOR PIKETON WASTE TESTS 1 AND 2 6-40

TABLE 6-22: SUMMARY OF PCDD EMISSION RATES FOR PIKETON WASTE TESTS 4 AND 5 6-42

TABLE 6-23: SUMMARY OF PCDF EMISSION RATES FOR PIKETON WASTE TESTS 4 AND 5 


\section{LIST OF TABLES}

(Continued)

Page

TABLE 6-24: MULTIPLE ORGANIC FEEDSTOCK RETEST RESULTS

SUMMARY - DESTRUCTION AND REMOVAL EFFICIENCY

$6-52$

TABLE 6-25: SUMMARY OF PCDD EMISSION RATES FOR MULTIPLE ORGANIC FEEDSTOCK - TEST R3.

TABLE 6-26: SUMMARY OF PCDF EMISSION RATES FOR MULTIPLE ORGANIC FEEDSTOCK - TEST R3 6-55

TABLE 6-27: 2,3,7,8-TCDD TEQ STACK EMISSIONS $6-57$

TABLE 6-28: CHEMICAL ANALYSIS OF BED RESIDUE 6-78

TABLE 6-29: RCRA METALS ANALYSIS OF BED RESIDUE. 6-78

TABLE 6-30: RESULTS FROM EDS 6-79

TABLE 6-31: GLASS COMPOSITIONS. $6-80$

TABLE 6-32: TCLP RESULTS 6-81

TABLE 7-1: LOW-LEVEL MIXED WASTE INVENTORY - 18 SITES $7-10$

TABLE 8-1: THERMOCHEM STEAM REFORMER CAPITAL COST

$8-2$

TABLE 8-2: THERMOCHEM STEAM-REFORMING - ECONOMIC SUMMARY ..... $\quad 8-4$ 


\section{ABSTRACT}

Under DOE Contract No. DE-AR21-95MC32091, Steam Reforming of Low-Level Mixed Waste, ThermoChem has successfully designed, fabricated and operated a nominal 90 pound per hour Process Development Unit (PDU) on various low-level mixed waste surrogates. The design, construction, and testing of the PDU as well as performance and economic projections for a 500$\mathrm{lb} / \mathrm{hr}$ demonstration and commercial system are described. The overall system offers an environmentally safe, non-incinerating, cost-effective, and publicly acceptable method of processing LLMW. The steam-reforming technology was ranked the No. 1 non-incineration technology for destruction of hazardous organic wastes in a study commissioned by the Mixed Waste Focus Area published April 1997.' The ThermoChem steam-reforming system has been developed over the last 13 years culminating in this successful test campaign on LLMW surrogates. Six surrogates were successfully tested including a 750-hour test on material simulating a PCB- and Uraniumcontaminated solid waste found at the Portsmouth Gaseous Diffusion Plant. The test results indicated essentially total (>99.9999\%) destruction of RCRA and TSCA hazardous halogenated organics, significant levels of volume reduction ( $>400$ to 1 ), and retention of radionuclides in the volume-reduced solids. Cost studies have shown the steam-reforming system to be very cost competitive with more conventional and other emerging technologies.

\section{Acknowledgments}

ThermoChem wishes to acknowledge and express thanks for the exceptional support of its subcontractors: MTCI, PDU fabrication and testing; Stone \& Webster Engineering, design and technical support; Thermatrix, flameless oxidizer and gas cleanup; and the Clemson Vitrification Research Laboratory, vitrification tests on bed inorganics. A special thank you to the FETC technical and procurement staff: William Huber, Cliff Carpenter, Richard Dennis, and Denise Riggi.

1 "Evaluation of Alternative Nonflame Technologies for Destruction of Hazardous Organic Waste," Schwinkendorf, W.E., Musgrave, B.C., Drake, R.N., prepared for the U.S. Department of Energy/Assistant Secretary for Environmental Management, DOE Idaho Operations Office, by Idaho National Engineering Laboratory, Mixed Waste Focus Area, Lockheed Martin Idaho Technologies Company, Idaho Falls, Idaho, April 1997. 


\section{EXECUTIVE SUMMARY}

The basic objective of low-level mixed waste treatment systems is to completely destroy the hazardous constituents and to simultaneously isolate and capture the radionuclides in a superior final waste form. The Department of Energy (DOE) is sponsoring the development of advanced technologies that meet this objective while achieving maximum volume reduction, lowlife cycle costs and maximum operational safety. Under DOE Contract No. DE-AR2195MC32091, Steam Reforming of Low-Level Mixed Waste, ThermoChem has successfully designed, fabricated and operated a nominal 90 pound per hour Process Development Unit (PDU) on six various low-level mixed waste surrogates. The design, construction, and testing of the PDU as well as performance and economic projections for a $500-\mathrm{lb} / \mathrm{hr}$ demonstration and commercial system are described.

The PDU consists of the following major subsystems:

- Solid/liquid feeding system

- First-stage fluidized bed steam reformer

- Particulate removal

- Flameless thermal oxidizer

- Gas cleanup

The first-stage steam reformer is an indirectly heated fluidized bed with steam as the fluidizing medium. The bed temperature is very closely controlled to ensure complete volatilization and partial steam reforming of all organic compounds and to ensure retention of radionuclides, including Cesium compounds in solid form, along with other inorganics such as RCRA heavy metals in the bed. Waste material is continuously fed to the steam reformer reactor. The inorganics left in the reactor, including radioactive material and heavy metals, are continuously removed for final disposal or recovery of materials. After particulate removal, the product gases from the steam reformer flow into a Thermatrix flameless thermal oxidizer. This non-incineration system converts the carbon monoxide, hydrogen and hydrocarbon vapors into water and carbon dioxide and acid gases.

Testing in the PDU on RCRA organic compounds has confirmed that the ThermoChem overall system will achieve up to $99.9999 \%$ DRE for the Principal Organic Hazardous Constituents (POHCs) found in DOE wastes. A gas cleanup system removes acid gases as dry salts followed by an activated carbon filter as a final polishing step. Negative pressure is maintained throughout the entire system. 
PDU tests have verified complete retention of Cesium and heavy metal compounds as a solid in the first-stage reactor bed materials and volume reductions typically of 10 to 1 and as high as 1,000 to 1 for certain high organic content wastes. Extensive PDU tests on five types of lowlevel mixed waste surrogates have been completed and a long-term test (750 hours) on a sixth Uranium- and PCB-contaminated solid waste surrogate was completed. The latter waste is a surrogate for an actual 3 million pound solid waste found at the Portsmouth Gaseous Diffusion Plant. Essentially 100 percent carbon conversion with the attendant large volume reductions (400 to 1), retention of all Uranium surrogate, and DRE of $99.9999 \%$ on the PCB simulant has been demonstrated. Potential recycling of the Uranium is being investigated. Follow-on efforts include detailed design and costing of a steam-reforming system for testing on radioactive waste at a DOE site. 


\section{SECTION 1.0}

\section{BACKGROUND}

\subsection{LOW-LEVEL MIXED WASTE AT DOE}

One of the major problems to be dealt with by the Department of Energy (DOE) in its efforts to achieve and maintain full compliance with all applicable laws and regulations is the present inventory and continuous production of low-level mixed waste (LLMW) at DOE sites. The current total inventory at DOE sites is in excess of $100,000 \mathrm{~m}^{3}$ (approximately 160,000 tons) with annual production of up to $20,000 \mathrm{~m}^{3}$. Most of this LLMW is stored in drums, barrels, and steel boxes at 20 different sites throughout the DOE complex.

The basic objective of LLMW treatment systems is to completely destroy the organic hazardous constituents or characteristics and to simultaneously isolate and capture the radionuclides and toxic metals in a stable, non-leachable final waste form. Historically, very few acceptable options have been available for the treatment of LLMW. Concerns over the safety of incineration systems have limited their applicability. An advanced treatment system is needed that totally destroys the hazardous components in LLMW without incineration and produces a nonleachable final waste form that can be readily and safely disposed of at a licensed low-level burial site. The possibility of radioisotope recovery and recycle is also being investigated. ThermoChem, having completed very successful tests on a variety of hazardous and radioactive surrogate wastes, is in the final stage of development of a steam-reforming system capable of treating a wide variety of DOE low-level mixed waste that meets these performance objectives.

Approximately 25 percent of the LLMW in the DOE inventory is comprised of material containing greater than 75 percent combustible organics thereby making it very compatible with the ThermoChem steam-reforming system. ${ }^{(1)}$ The ThermoChem steam-reforming technology was selected for further development by the DOE under a competitive procurement administered by the Morgantown Energy Technology Center, now the Federal Energy Technology Center. The 
was awarded in June 1995. This Topical Report presents the results of the tasks performed under that contract.

\subsection{THERMOCHEM STEAM REFORMING}

\subsubsection{Technology Description}

Under DOE Contract No. DE-AR21-95MC32091, Steam Reforming of Low-Level Mixed Waste, ThermoChem has successfully designed, fabricated and operated a nominal 90 pound per hour Process Development Unit (PDU) on various low-level mixed waste surrogates. The PDU flow diagram is depicted in Figure 1-1.

A ThermoChem steam-reforming system configured for treatment of LLMW in either liquid or solid form with halogenated organics (including PCBs) and heavy metals and radioactive elements would consist of the following major subsystems:

- Solid/liquid receiving, sorting, feeding subsystem,

- Indirectly heated fluidized bed steam reformer with internal cyclone,

- HEPA filter for fines capture,

- Flameless thermal oxidizer,

- Gas cleanup containing:

- spray cooler/dry scrubber for acid gas removal

- caustic feed system for scrubber

- baghouse and HEPA filters for solid salts removal

- polishing wet scrubber for acid gas

- activated carbon filter for mercury removal

- induced draft blower,

- Boiler and superheater,

- Solid residue treatment/packaging system,

- Exhaust stack and continuous emissions monitoring system, and

- Instrumentation and controls.

A general description of each of these subsystems and their functions is provided in the following sections. 


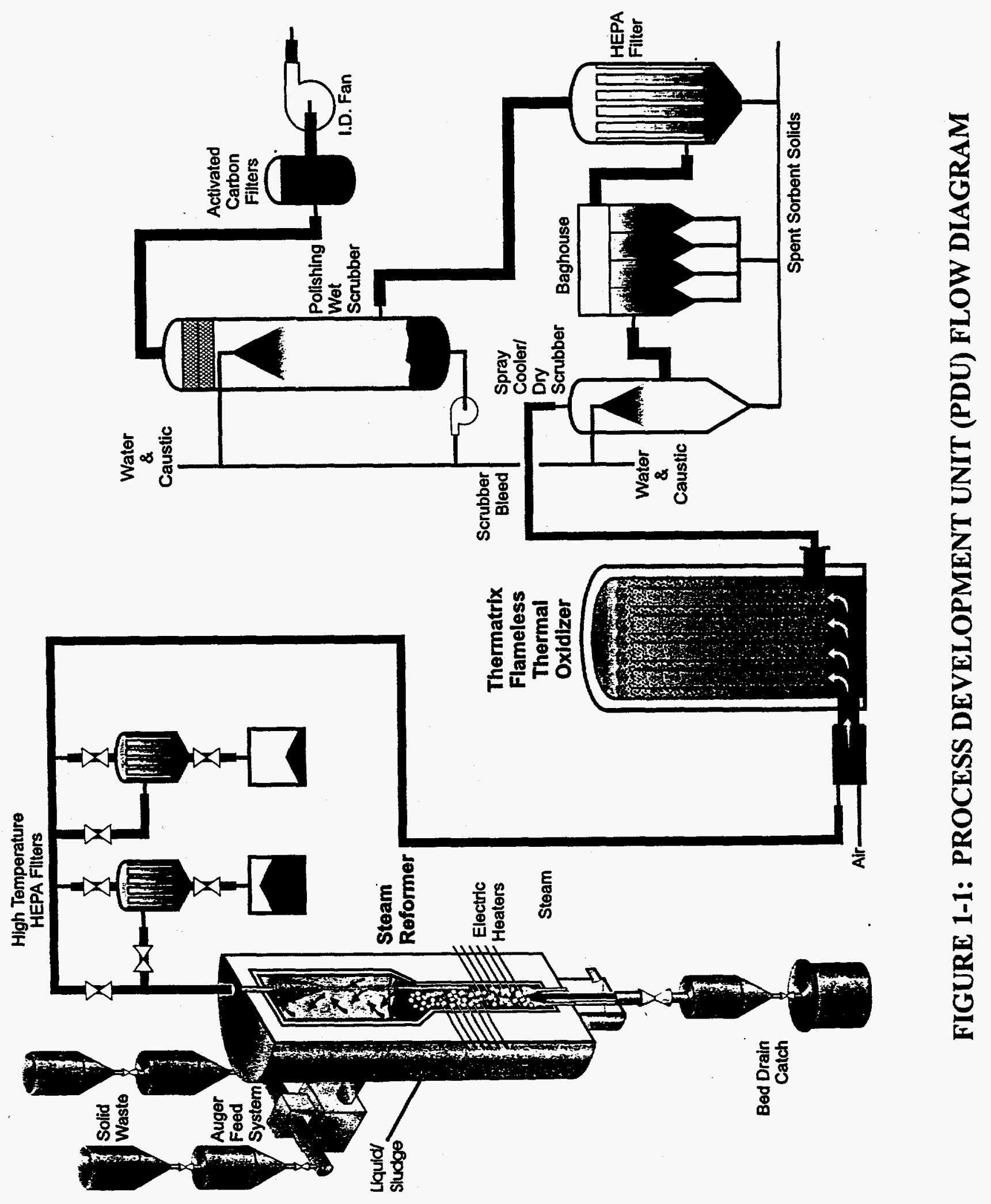




\section{Solid/Liquid Receiving, Sorting, Feeding Subsystem}

The initial screening and characterization step would be based on review of inventory documentation for each container to identify general waste characteristics. Receipt inspection also verifies the suitability of the shipping container for further handling and staging within the process through verification of its integrity and the absence of deterioration or evidence of leaks. Radiation detection and manifest verification will be provided by hand-held detectors appropriate for the radionuclides expected, or that could be found, in the waste.

It is anticipated that initial sorting operations, if required, would be performed in a glovebox environment with containers loaded through air-lock doors with a drum handler. The glovebox would be provided with a sorting table designed to capture and collect aqueous wastes and provides lighting, air and power connections for tools. Hoisting means would provided up to and within the glove box.

Sorted, combustible solid waste would be reduced to a uniform size in a commercialgrade, two-staged chopper/shredder for more efficient handling and steam reforming. Besides providing a uniform size of waste (nominally less than 2 inches), the shredding process also serves to homogenize the waste materials such that the feedstock has a more uniform density, heating value, inorganic fraction, etc. Waste is fed to the shredder mechanism through an air-lock to contain dust and fines during the shredding process. Subsequent to shredding, the feedstock is transferred to surge storage leading to the reactor. The surge storage affords a further capability to blend individual batches of prepared waste. The prepared waste stream is fed directly into the steam reformer by a water-cooled screw conveyor assembly.

All material-handling systems are maintained under negative pressure to maximize containment, dust and contaminant control. Fire prevention is accomplished by administrative controls (such as sorting procedures) and design features, including the exclusion of spark sources and installation of a fire suppression system. 
An area radiological monitoring subsystem would be provided for safeguarding worker, public, and environmental health and safety. Equipment will be provided to monitor the treatment area and any other areas where radioactive materials could be encountered to ensure that contamination levels in these areas are kept well within established limits. Area monitors would be located at work stations and in areas where radioactive material is expected to temporarily be accumulated. Monitors would be installed at all system discharge points to continuously monitor the radioactivity, among other constituents, as required by regulatory commitment and a higher commitment to worker and public health and safety.

\section{Indirectly Heated Fluidized Bed Steam Reformer}

The steam reformer is an indirectly heated fluidized bed in a refractory-lined reactor vessel. The apparatus and processes are covered by MTCI Patent Nos. 5,059,404; 5,211,704; and $5,133,297$. The technology has been successfully demonstrated on a very wide range of chlororganic wastes and is being applied commercially in the processing of black liquor in the paper industry. A 50 ton per day demonstration project was recently completed at a Weyerhaeuser paper mill in New Bern, North Carolina. ThermoChem has exclusive licensing rights to design, manufacture, market and apply the MTCI steam-reforming technology.

The nominal $90 \mathrm{lb} / \mathrm{hr}$ PDU tested under DOE contract is a reactor 42 inches in diameter with an active bed volume of 1 square foot by 6 feet. The remaining volume is refractory. The fluidizing medium is steam for superior mixing and heat transfer to the material to be treated. Electrical heaters immersed in the bed provide supplemental heat as required. The PDU heaters provide up to $40 \mathrm{KW}$ of electrical heating. The bed temperature is very closely controlled to ensure complete volatilization and partial steam-reforming of all organic compounds and to ensure retention of radionuclides including Cesium compounds in solid form along with other inorganics in the bed. If Cesium is not in the feed material, the bed will be controlled at approximately $1350^{\circ} \mathrm{F}$ and essentially all of the carbon will be steam reformed, i.e., removed as synthesis gas. If Cesium is present, the bed temperature will be controlled at $1050^{\circ} \mathrm{F}$ to ensure retention of Cesium in the solid bed material. This temperature will result in approximately 65 to 98 percent carbon conversion with the remaining carbon exiting in solid form in the bed drain and high-temperature 
HEPA (HT HEPA) catch. The carbon conversion depends on the temperature as well as the composition of the organic material. Pure polypropylene and polyethylene, for example, result in essentially 100 percent carbon conversion. The carbon can either be disposed with the solid radioactive waste (volume reductions are still greater than 10 in most cases) or small amounts of air can be added to the bed with the fluidizing steam in the absence of waste feed and the carbon will be converted to carbon monoxide. Even though air is being added, the bed stays reducing and the temperature is closely controlled to $1050^{\circ} \mathrm{F}$ by reducing power to the electrical heaters.

The first-stage steam reformer is continuously fed waste material using a positive displacement pump subsystem for liquids and slurries and/or a metering screw feeder with lock hoppers for solids. The screw feeder is also used to add glass frit or sand to the bed as needed if the final waste form is to be glass. Sand or glass frit is also used as the start-up bed material. The inorganics left in the reactor, including radioactive material, are continuously removed and collected in sealed containers. The bed material will be a nominal 250 -micron mean size material. The final waste form can be glass, metal or a high-integrity container, depending upon radiation levels and leachability requirements. Polyethylene encapsulation or a simpler non-thermal, immobilization process is anticipated to meet all disposal requirements for most DOE LLMW.

An internal cyclone and high-temperature pulse jet HEPA filters at the exit of the steam reformer serve to essentially eliminate the carryover of particulates in the synthesis gas stream. The HEPA filters are silicon carbide ceramic matrices that operate effectively at approximately $1000^{\circ} \mathrm{F}$. Two HEPA filters in series provide redundancy and can actually be operated in series or parallel. The PDU tests showed that the filters achieved greater than 99.97 percent particulate removal at 0.3 microns.

\section{Thermatrix Flameless Oxidizer}

The particulate-free synthesis gas from the steam reformer/HEPA filters flows into the Thermatrix flameless thermal oxidizer (FTO). The FTO is a non-incineration system that converts the carbon monoxide, hydrogen and hydrocarbon vapors into water vapor, carbon dioxide, and acid gases. The FTO technology grew out of research conducted by Lawrence Livermore 
National Laboratory for oil shale processing. The FTO technology has been successfully applied, with over 60 commercial units currently in operation throughout the world, with installations at companies including: DOE, DOW, FMC, Mobil, Monsanto, Shell, General Electric and Georgia Pacific. The FTO technology has also been successfully demonstrated on $\mathrm{TCE}^{(2)}$ at DOE's Savannah River site, the Idaho National Engineering Laboratory, and McClellan Air Force Base. ${ }^{(3)}$ Thermatrix has exclusive licensing rights to design, manufacture, market, and apply the FTO technology.

The FTO technology when coupled with the steam reformer process is an ideal matching of technologies. The FTO technology as a stand-alone technology on gas vapors has consistently achieved DREs of $99.99+\%$ and as part of a PCB soil remediation system at a superfund site in the Northeastern United States, achieved $99.99999 \%$ DRE. ${ }^{(4)}$

The oxidizer is an insulated vessel packed with ceramic material according to a proprietary matrix configuration. At start-up, the ceramic matrix is preheated to between 1600 and $1800^{\circ} \mathrm{F}$. Once the preheating has been completed, the synthesis gas from the steam reformer containing carbon monoxide, unconverted hydrocarbons, hydrogen and steam is premixed with excess air and the oxygen-rich $800^{\circ} \mathrm{F}$ mixture is introduced into the oxidizer. The fume stream first passes through a mixing zone and then through the reaction zone of pre-heated ceramic material where complete flameless oxidation occurs in a fraction of a second. The oxidation process releases heat that is absorbed and conserved by the ceramic matrix which helps maintain consistent oxidation temperatures between $1650^{\circ} \mathrm{F}$ and $2000^{\circ} \mathrm{F}$. The expansion of the gases as they heat up is accommodated by an increase in the ceramic matrix void volume. Due to its flameless design and consistent temperature profile, the oxidizer achieves very high DRE on gas streams with heating values as low as $10 \mathrm{Btu} / \mathrm{scf}$ with de minimis $\mathrm{NO}_{\mathrm{x}}$ formation ( $\left.2 \mathrm{ppm}\right)$. The Thermatrix system also has no dioxin emissions because of excess oxygen (at least $3 \%$, more commonly $11 \%$ ), greater than $1600^{\circ} \mathrm{F}$, no possible bypassing of the reaction zone, and elimination of cold surfaces in the exhaust flow path. The Thermatrix oxidizer is exceptionally safe and is even certified for use in highly flammable environments where conventional systems are prohibited. ${ }^{(5)}$ 


\section{Gas Stream Cleanup}

The exhaust of the flameless thermal oxidizer (FTO) may contain sulfur dioxide and hydrochloric acid from sulfur and chlorine compounds originally in the LLMW. A gas cleanup system removes acid gases as dry salts. The exhaust gas from the FTO will be connected to a gas-cleaning system. The gas-cleaning system includes an evaporative cooler for gas cooling, partial acid gas removal, and salt drying, a medium temperature $\left(350^{\circ} \mathrm{F}\right)$ pulse jet baghouse for salt recovery, a HEPA filter for final particulate control, a packed bed scrubber with integral mist eliminator for complete acid gas control, tandem-activated carbon beds for mercury control, a high-pressure vacuum blower for system draft, and an exhaust stack with continuous emission monitoring system (CEM). The liquid blowdown from the wet scrubber is fed forward and used as the quench water in the evaporative cooler. This results in zero liquid effluent from the entire system and produces a dry residue salt for ultimate disposal. This gas-cleaning system in concert with the high performance of the FTO process will result in a clean flue with actual emission rates of particulate matter, acid gases, regulated metals, radionuclides, and organics well within all applicable emissions regulations.

\section{Auxiliary Equipment}

Negative pressure is maintained throughout the entire system by an induced draft blower or steam eductor. The zero pressure point is at the bottom of the fluid bed at the solids removal point. The vacuum blower is pulling approximately 6 psia at the suction side. A natural gas-fired boiler and superheater would supply steam to the steam reformer with heat supplemented from the flameless oxidizer. Before being released to the atmosphere, the gas would be monitored continuously for radioactivity and TSCA/RCRA license requirements. The system would also incorporate comprehensive instrumentation and controls to facilitate seamless. start-up, safe operation, process monitoring and control, and shutdown.

\section{Final Solid Waste Storage/Disposal}

Detoxified solid residue from the steam reformer is removed from the reactor into transfer containers for removal to primary stabilization or storage. The solids will contain inorganic matter from the LLMW including radioisotopes as confirmed by PDU testing, sand if added for 
vitrification, and small amounts of carbon. Waste from the treatment operation will be stored pending final testing results, then further treated and packaged consistent with applicable waste acceptance criteria. The final packages will be visually examined, weighed, tagged, logged, and recorded as part of a complete assay and certification operation. In the assay-and-certification operation, the containers are examined by radioassay and other detection devices to ensure their compliance with the applicable transportation, storage, and disposal criteria.

Secondary waste streams and segregated wastes (e.g., from initial sorting) are handled similarly to ensure full compliance with regulatory waste acceptance criteria as well as the project objective of reduced waste volume and lower disposal costs.

\subsubsection{Applicability of ThermoChem Steam Reforming to LLMW Treatment}

There exist many technologies for the treatment of mixed wastes. ${ }^{(6,7)}$ The technology choice however will depend upon such factors as waste type, waste composition, treatment effectiveness, regulatory compliance, environmental health and safety impact, cost-effectiveness, technology maturity and public acceptance.

The treatment technologies can be broadly grouped into two categories, viz. thermal and non-thermal. Schwinkendorf ${ }^{(7)}$ has presented a critical review of the current and innovative treatment technologies. He has also reported the results of system studies conducted to evaluate costs, performance and technology maturity. The study evaluated 20 thermal systems, 5 nonthermal systems (operating temperature $<660^{\circ} \mathrm{F}\left[350^{\circ} \mathrm{C}\right]$ ) and 5 enhanced non-thermal systems. The basic parameters for the study were: $2,927 \mathrm{lb} / \mathrm{hr}$ waste feed, $60 \%$ unit availability, 20-year operation, 2.36 million pounds of waste treatment, and final disposal at $\$ 243 / \mathrm{ft}^{3}$. The major results from this study were:

- Life-cycle costs for non-thermal systems were 40 to $60 \%$ higher than those for thermal systems.

- Volume reduction from thermal systems was about 3 times superior to those from non-thermal systems. 
- Thermal systems however efflux off-gas at significantly higher rates than non-thermal systems.

- Thermal systems require about $30 \%$ less disposal site area than nonthermal systems.

The author concludes, "Non-thermal systems are immature and ability to meet regulations is uncertain."

This study also presented detailed results for four thermal systems given below:

- Rotary kiln with air and vitrification of ash,

- $\quad$ Slagging kiln,

- Plasma furnace, and

- Steam reforming of combustible waste and vitrification of non-combustible waste.

The life-cycle cost and volume reduction were comparable and only a little different for the four systems. The steam reformer, however, resulted in the lowest life-cycle cost overall. Additionally, the off-gas efflux rate from the steam reformer and plasma furnace were an orderof-magnitude smaller than those for the two kiln systems. All these findings are very encouraging indeed and attest to the superiority of the steam-reforming technology for the treatment of mixed wastes.

The ThermoChem/Thermatrix steam-reforming and flameless oxidation system offers the following benefits:

- Facilitates the separation of the radioactive and hazardous components and in turn enables the application of the most appropriate treatment step for each of the two groups.

- Thermal treatment of the hazardous species in a reducing environment decomposes and reforms the organic matter, generates a smaller off-gas stream, and avoids dioxin/furan formation. 
- Lower temperature (900-1350 $\mathrm{F})$ and lower off-gas rate permit the use of ceramic barrier filters for hot gas cleanup and result in minimal particulate carryover and thermal penalty.

- The steam reformer is feedstock flexible and can accept a variety of feedstocks including liquids, sludges, slurries, soils and debris of differing chemistry.

- The thermal oxidizer ensures complete oxidation of the syngas and in turn greater than $99.99 \%$ destruction and removal efficiency (DRE). This DRE is basically limited only by detection limits in the exhaust of the material being destroyed. For example, PCB DRE exceeds $99.9999 \%$.

- Competent treatment of oxidizer off-gas results in negligible air pollution and in turn minimizes public and occupational health risks. The stack gases are all essentially $\mathrm{N}_{2} \mathrm{O}_{2}, \mathrm{H}_{2} \mathrm{O}$ and $\mathrm{CO}_{2}$.

- The steam reformer facilitates significant volume reduction through the removal of water, organics, chlorine, sulfur and nitrogen. The non-hazardous residue is drained hot and forms an ideal feed for either vitrification or molten metal processing. The residue is also amenable for stabilization/immobilization through processes such as phosphate-bonded ceramic forming and polymer microencapsulation. In most cases, burial in a high-integrity container is likely to meet all applicable regulations.

- The off-gas treatment train generates only dry spent solids and operates in the zero-liquid discharge mode. Due to efficient gas-solid contact, the secondary waste generation rate is minimized.

- The whole system operates at below atmospheric pressure to minimize accidental exposure and maximize personnel safety.

- The non-incinerator mode of operation can extinguish stakeholder concerns and facilitate ease of permitting. 
- The potential for radionuclide emissions is minimized due to low temperature $\left(900-1350^{\circ} \mathrm{F}\right)$ operation of the steam reformer and the consequent low volatility of the radionuclides. The species are essentially retained in the reformer bed solids and are drained for subsequent disposal.

- The process configuration permits continuous operation, high throughput and a high degree of automation. This reduces the time required for waste treatment and further helps to lower life-cycle costs.

- The system geometry affords modular design and relocatability. This has the potential to reduce construction time and also aid in treating wastes at multiple sites with the same unit.

- Both ThermoChem and Thermatrix have been offering steam reformers and flameless thermal oxidizers commercially and have diverse field demonstration experience. In fact, the units envisaged for this application are small in comparison to the large units offered commercially and therefore scale-up is a non-issue.

\subsubsection{Development Status}

ThermoChem, Inc. engages in the commercialization of a number of patented and proprietary technologies for applications worldwide. The company's systems include a versatile steam-reforming technology that can meet a broad spectrum of industrial needs. A significant market application of the steam-reforming system is chemical and energy recovery from spent processing liquors in the pulp and paper industry. The steam reformer also offers advantages for production of energy in the form of process steam, electricity, product gas, or solid fuels from paper mill sludge, bark or other wood waste or other biomass feedstock, and for other processes such as treatment of chlorinated effluents from bleaching.

ThermoChem is currently marketing its PulseEnhanced ${ }^{\mathrm{TM}}$ steam-reforming system to pulp and paper manufacturers on a build, own and operate basis as well as direct sales. 
According to the American Forest Products Association, worldwide chemical pulp and paper production is expected to yield approximately 230 million short tons of black liquor solids in 1997. About one-third of the chemical pulp mills currently in the company's overseas market area have no chemical recovery systems in place.

The ThermoChem steam-reforming system is the product of over 13 years of research, development and demonstration. The first tests of spent liquors were conducted in 1989 and pilot-scale processing began in 1993. In 1995, ThermoChem completed construction and test operations of a 50 ton/ day demonstration system at a Weyerhaeuser mill in New Bern, North Carolina. The demonstration program verified the operability, safety, reliability and efficiency of the steam-reforming system over a wide range of operating conditions. The steam-reforming system demonstrated superior chemical and energy recovery and experienced no operational problems during a 500-hour continuous test run.

The successful tests at the PDU scale as reported herein represent a significant step toward full commercial application of the ThermoChem steam-reforming system toward treatment of LLMW in the DOE complex.

In 1994, ThermoChem demonstrated its sludge processing system at a one ton per hour pilot plant in California. The plant met permitting requirements for the state of California and ran successfully for over 3,000 hours on paper mill sludge including chlorinated hydrocarbon plastics separated from the recycled paper process, and black liquor including a 500-hour continuous run.

Thermatrix has fabricated, installed and is operating over 60 commercial systems throughout the world. Table 1-1 lists some of these systems. As can be seen, the Thermatrix systems are operating on a very wide range of hazardous vapors. 
According to the American Forest Products Association, worldwide chemical pulp and paper production is expected to yield approximately 230 million short tons of black liquor solids in 1997. About one-third of the chemical pulp mills currently in the company's overseas market area have no chemical recovery systems in place.

The ThermoChem steam-reforming system is the product of over 13 years of research, development and demonstration. The first tests of spent liquors were conducted in 1989 and pilot-scale processing began in 1993. In 1995, ThermoChem completed construction and test operations of a 50 ton/ day demonstration system at a Weyerhaeuser mill in New Bern, North Carolina. The demonstration program verified the operability, safety, reliability and efficiency of the steam-reforming system over a wide range of operating conditions. The steam-reforming system demonstrated superior chemical and energy recovery and experienced no operational problems during a 500-hour continuous test run.

The successful tests at the PDU scale as reported herein represent a significant step toward full commercial application of the ThermoChem steam-reforming system toward treatment of LLMW in the DOE complex.

In 1994, ThermoChem demonstrated its sludge processing system at a one ton per hour pilot plant in California. The plant met permitting requirements for California and ran successfully for over 3,000 hours on paper mill sludge including chlorinated hydrocarbon plastics separated from the recycled paper process, and black liquor including a 500-hour continuous run.

Thermatrix has fabricated, installed and is operating over 60 commercial systems throughout the world. Table 1-1 lists some of these systems. As can be seen, the Thermatrix systems are operating on a very wide range of hazardous vapors. 
TABLE 1-1:

THERMATRXX ${ }^{\circledR}$ FLAMELESS THERMAL OXIDATION SYSTEMS INSTALLATION LIST

\begin{tabular}{|c|c|c|c|c|c|c|c|}
\hline $110 \%$ & NDUSTRK & 100ATrox & APPLATHOH & 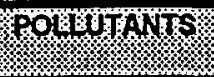 & XODE & $\begin{array}{l}\text { xoow } \\
\text { sonM }\end{array}$ & WSTE \\
\hline 1 & $\begin{array}{l}\text { Automotive Paint } \\
\text { Finishing } \\
\text { (prototype) }\end{array}$ & Michigan & $\begin{array}{l}\text { Paint Solvent } \\
\text { Emission }\end{array}$ & $\begin{array}{l}1,000 \text { ppmv of } \\
\text { paint solvent }\end{array}$ & GR-6500 & 6500 & 1994 \\
\hline 2 & $\begin{array}{l}\text { Specialty } \\
\text { Chemicals }\end{array}$ & Pennsytrania & $\begin{array}{l}\text { Soil Vapor } \\
\text { Extraction } \\
\text { (remediation) }\end{array}$ & $\begin{array}{l}\text { Chlorinated } \\
\text { vOCs from Soll } \\
\text { Vapor Extraction }\end{array}$ & GS-150 & 150 & $\begin{array}{l}\text { Mid } \\
1995\end{array}$ \\
\hline 3 & Chemical & Texas & $\begin{array}{l}\text { Chlorinated Air } \\
\text { Stripper } \\
\text { Off Gas }\end{array}$ & $\begin{array}{l}\text { Chloroform } \\
\text { Acetone }\end{array}$ & GSC-600 & 600 & $\begin{array}{l}\text { Late } \\
1996\end{array}$ \\
\hline 4 & Environmental & California & Soll remediation & $\begin{array}{l}\text { VOCs from } \\
\text { Thermal } \\
\text { Desorber }\end{array}$ & GR-30000 & 30,000 & $\begin{array}{l}\text { Earty } \\
1996\end{array}$ \\
\hline 5 & Environmental & $\begin{array}{l}\text { British } \\
\text { Columbia }\end{array}$ & Soil remediation & $\begin{array}{l}\text { VOCs from } \\
\text { Thermal } \\
\text { Desorber }\end{array}$ & GR-12000 & 12,000 & $\begin{array}{l}\text { Earty } \\
1996\end{array}$ \\
\hline 6 & Aerosol & North Carolina & $\begin{array}{l}\text { Aerosol Process } \\
\text { Vents }\end{array}$ & VOCs & GS-2500 & 2500 & $\begin{array}{l}\text { Mid } \\
1996\end{array}$ \\
\hline 7 & Chemical & United Kingdom & Process Vent & $\begin{array}{l}\text { Methyl Chloride, } \\
\text { Ethyl Chloride, } \\
\text { Ethanol, Carbon } \\
\text { Tetrachloride, } \\
\text { and Chlorine }\end{array}$ & GSC-750 & 750 & $\begin{array}{l}\text { Mid } \\
1996\end{array}$ \\
\hline 8 & Hydrocarbons & Netherlands & Tank Vents & VOCs, Benzene & GS-500 & 500 & $\begin{array}{l}\text { Late } \\
1996\end{array}$ \\
\hline 9 & $\begin{array}{l}\text { Chemical } \\
\text { (Herblcides) }\end{array}$ & Maryland & $\begin{array}{l}\text { Process Vent } \\
\text { (balch process) }\end{array}$ & $\begin{array}{l}\text { Various } \\
\text { chlorinated and } \\
\text { fluorinated } \\
\text { VOCs }\end{array}$ & GSC-4500 & 4500 & $\begin{array}{l}\text { Earty } \\
1996\end{array}$ \\
\hline 10 & Pulp and Paper & Mississippl & Process Vent & $\begin{array}{l}20,000 \text { ppmv } \\
\text { total of } \\
\text { sulfonated vOC }\end{array}$ & GS-3000 & 3000 & $\begin{array}{l}\text { mid } \\
1995\end{array}$ \\
\hline 11 & Environmental & Idaho & $\begin{array}{l}\text { Waste Oil } \\
\text { Recycling } \\
\text { Process Vent \& } \\
\text { Process Heat }\end{array}$ & VOCs & GS-4000 & 4000 & $\begin{array}{l}\text { late } \\
1994\end{array}$ \\
\hline 12 & Environmental & Europe & $\begin{array}{l}\text { Waste Oil } \\
\text { Recycling } \\
\text { Process Vent } 8 \\
\text { Process Heat }\end{array}$ & VOCs & GS-4000 & 4000 & $\begin{array}{l}\text { Late } \\
1995\end{array}$ \\
\hline 13 & $\begin{array}{l}\text { Dept of Energy } \\
\text { (DOE) }\end{array}$ & Idaho & $\begin{array}{l}\text { Soll Vapor } \\
\text { Extraction off- } \\
\text { gas treatment }\end{array}$ & $\begin{array}{l}\text { Chlorinated } \\
\text { vocs }\end{array}$ & $\begin{array}{l}1-G R-200 \\
2-G R-400\end{array}$ & $\begin{array}{l}1-200 \\
2-400\end{array}$ & $\begin{array}{l}\text { Late } \\
1995\end{array}$ \\
\hline 14 & Dept. of Defense & California & $\begin{array}{l}\text { Groundwater } \\
\text { remediation }\end{array}$ & CVOCs & GR250 & 250 & $\begin{array}{l}\text { Aug. } \\
1997\end{array}$ \\
\hline 15 & $\begin{array}{l}\text { Technology } \\
\text { Development }\end{array}$ & New York & $\begin{array}{l}\text { PCB Site } \\
\text { Remediation in } \\
\text { conjunction with } \\
\text { Shell thermal } \\
\text { blanket } \\
\text { technology }\end{array}$ & PCBs & GR-2000 & 2000 & $\begin{array}{l}\text { Late } \\
1995\end{array}$ \\
\hline 16 & $\begin{array}{l}\text { Remediation / Five } \\
\text { site, four state } \\
\text { demo }\end{array}$ & TX, NY, FL, OH & $\begin{array}{l}\text { Soil Vapor } \\
\text { Extraction } \\
\text { off-gas } \\
\text { treatment }\end{array}$ & $\begin{array}{l}\text { Chlorinated } \\
\text { VOCs in SVE } \\
\text { off-gas }\end{array}$ & GS-150 & 150 & $\begin{array}{l}\text { Early } \\
1996\end{array}$ \\
\hline
\end{tabular}




\section{SECTION 2.0}

\section{PROGRAM OBJECTIVES AND SCOPE}

\section{$2.1 \quad$ PROGRAM OBJECTIVES}

The objective of the work under DOE Contract No. DE-AR21-95MC32091 is to evaluate the performance of the ThermoChem, Inc./Manufacturing and Technology Conversion International, Inc. (MTCI) steam-reforming technology for the processing and volume reduction of low-level mixed waste (LLMW). This performance evaluation is to provide information regarding the capabilities, cost-effectiveness, and safety of this remediation process compared to other technology options.

ThermoChem was to evaluate the performance of the ThermoChem/MTCI steamreforming technology at a Process Development Unit (PDU) scale. The work was limited to testing of approved mixed waste surrogates. ThermoChem was to analyze the PDU performance results. Based on these results, ThermoChem was to provide an evaluation of the capabilities, safety, permitting, and cost-effectiveness of the steam-reforming process at both the PDU and pilot/ demonstration-unit scales of operation. An economic evaluation of a pilot/demonstrationscale unit was also performed. A limited effort, preliminary conceptual design of a pilot/ demonstration-scale unit, which shall operate on actual mixed waste, was also performed to support the economic evaluation.

In the event a positive decision is made by DOE, ThermoChem will proceed with the optional tasks to perform an in-depth conceptual design of a steam-reforming unit at the pilot/ demonstration plant scale to operate on actual mixed waste. ThermoChem shall recommend site(s) that are suitable for field demonstration of the unit and perform an economic evaluation of the unit's projected performance. 


\subsection{WORK TASKS}

\section{Task 1.1: Surrogate Feedstock Identification and Preparation}

ThermoChem was to identify at least seven surrogate feedstocks for use in testing and describe these feedstocks along with the criteria and process through which the feedstocks were selected. An independent chemical analysis was to be made of the feedstocks and certified by the laboratory providing the services.

\section{Task 1.2: $\quad$ Test Plan}

ThermoChem was to prepare and submit a definitive Test Plan. The Test Plan shall include, but not necessarily be limited to, a detailed description of all tests to be performed including testing conditions, tests duration, parameters to be varied, including ranges and specific unit performance goals and success criteria.

The Test Plan shall be based on a PDU capacity of at least 30 pounds per hour. The Test Plan shall include a minimum of 400 hours of PDU operating including unit pre-heat, feeding, and cool-down times. Initial unit shakedown is not to be considered as part of the 400 operating hours. The Test Plan shall include at least 160 hours of unit feeding as part of the 400 operating hours.

Along with the defined matrix of experiments, the Test Plan shall also define the analysis procedures including a detailed description of all measurements and characterizations to be performed and any necessary instrumentation calibration procedures. The sample points, number of samples, and amounts of sample to be withdrawn shall be described along with sampling procedures and apportionments. The organizations performing the analytic analysis of the samples shall also be identified.

The Test Plan shall also include an assessment of the accuracy of the tests measurements in correctly indicating the actual operation and performance of the unit including identification, assessment, and quantification of significant sources of error. The Test Plan shall also provide a 
summary of operating and safety procedures including the plan for disposal of any remaining surrogate feedstock.

\section{Task 1.3: Process Development Unit Preparation and Shakedown}

ThermoChem was to prepare the PDU system based on the Test Plan. All necessary construction including instrumentation, sample ports, and mechanisms for safely withdrawing samples during operation was to be performed in accordance with the Test Plan.

ThermoChem was to perform all necessary instrumentation calibrations as well as shakedowns of all major subsystems including safety interlocks and start-up and shutdown procedures prior to full system operation. ThermoChem was to maintain records of all calibration and subsystem checkouts and procedures.

\section{Task 1.4: $\quad$ Process Development Unit Testing}

ThermoChem was to conduct PDU tests in accordance with the Test Plan. Logs shall be kept of all data collected including any test anomalies or incidents and a complete record of operation from start-up to shutdown. Test samples shall be sealed in containers bearing test, time, withdrawal position, and other pertinent information as described in the Test Plan.

\section{Task 1.5: Data Analysis}

ThermoChem was to perform a systematic reduction of all data obtained during each test and integrate that data with the certified chemical analysis from the independent laboratories to characterize each waste stream and the disposition of each component thereto. Heat and mass balances with reasonable closure were to be prepared, evaluated and analyzed with respect to any data anomalies. Utilizing the data, projections of the disposition of components that will be present in an actual mixed waste stream shall be made to define the interfaces required by the degree of organic destruction, degree of waste reduction, distribution of components in each waste stream, and their disposition within and to the DOE waste management system. 


\section{Task 1.6: Preliminary Conceptual Design}

ThermoChem was to perform a limited-effort preliminary conceptual design of a larger, demonstration-scale steam reformer system. The preliminary conceptual design shall only be performed to a level of detail necessary to support the economic evaluation. The preliminary design shall be based on a six tons per day capacity unit but may be slightly altered based on prior task results, design considerations, and economic evaluation. ThermoChem is to notify the COR of any alteration in the design capacity. Similarly, the design shall be based on a transportable unit but may also be revised to a stationary unit based on the same considerations and COR notification.

ThermoChem was to define the functional requirements of the unit and use these requirements in the development of a Basis of Design. Sites with potential interest shall be contacted to determine interest and preliminary user requirements of the unit. The basis shall include a summary of potential site locations and conditions, description of wastes to be treated, equipment design factors, limits for site release of wastes, utility conditions, civil and structural design basis, chemical requirements, environmental requirements, and a process description.

ThermoChem was to prepare the preliminary conceptual design which shall include, but not necessarily be limited to, the process flow diagrams, heat and material balances, approximately sized major equipment, estimates of utility requirements, and the estimated footprint for the plant. The conceptual design shall include preliminary consideration of all major subsystems including the steam reformer, feed-handling and prep subsystem, effluent treatment and conditioning subsystem, waste packaging and stabilization subsystem, and the chemical and radiation monitoring subsystem. Transportation, set-up, shakedown and take-down costs shall also be considered. The conceptual design shall include the identification of the most attractive site(s) for demonstration of the technology along with any obtained documentation indicating site support of the technology. This documentation may include, but not necessarily be limited to, memorandums of understanding, letters of intent, contracts and agreements. 
As part of the conceptual design, ThermoChem was to prepare a factored cost estimate for the facility including estimated range of error of the cost estimate. The operating costs along with any other major life-cycle cost of the facility shall also be estimated along with an associated estimate of range of error.

\section{Task 1.7: $\quad$ Economic Evaluation}

ThermoChem was to prepare an economic evaluation of a commercial plant steam reformer system based on the prior tasks results including the preliminary conceptual design of the demonstration scale unit. Economic comparisons shall be made with other disposal methods including competing and/or similar technologies. The economic evaluation shall include benchmarking of the costs of disposal by conventional technologies and the reduction in costs associated with utilization of the ThermoChem/MTCI steam-reforming process. All benefits of the technology shall be fiscally quantified to the extent possible. ThermoChem shall collect the capital, operating and maintenance cost estimates and develop a unit cost for the destruction/ reduction of the waste feed stream. Projected modifications and cost differences between the pilot/demonstration unit and a commercial unit shall be described in detail. 


\section{SECTION 3.0 \\ SURROGATE IDENTIFICATION AND PREPARATION}

Seven different surrogate feedstocks representing a diverse spectrum of waste streams in the DOE complex were identified and selected. The seven feedstocks were:

- Heterogeneous debris;

- Aqueous halogenated organic liquids;

- Absorbed aqueous organic liquids;

- High organic content sludge;

- $\quad$ Cement, sludge, ash, and solids;

- Natural aqueous waste;

- Piketon waste, PCB and Uranium-contaminated solid.

The first six surrogate feedstocks were suggested by Sandia National Laboratory staff and in part by staff from the Idaho National Engineering Laboratory. The seventh feedstock was selected to generate detailed and longer term test data on a surrogate representative of a waste stream at a DOE site that could be a prime candidate for technology demonstration.

A summary of the first six formulations is given in Table 3-1. The seventh feedstock composition is furnished in Table 3-2. As can be seen, the surrogates contained RCRA organics, radionuclides, RCRA metals and bulk ingredients. The ultimate analyses for the six feedstocks as well as for the nominal composition of the seventh feedstock are provided in Table 3-3. The detailed descriptions of surrogate formulation and feedstock preparation are included as Appendix A. 
TABLE: $3-1$ :

SURROGATE FORMULATION - SUMMARY

FORMULATION NO.:

1

2

3

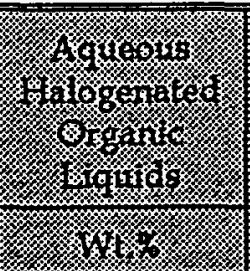

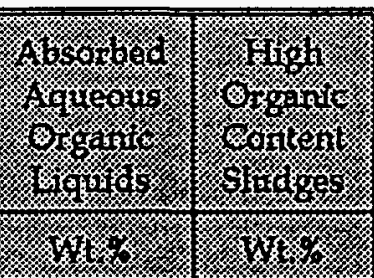

Bulk Ingredient

1. Activated Carbon

2. Cation Exchange Resin

3. Water

4. Wood

5. Polyvinylchloride (PVC)

6. Neoprene

7. Mild

Steel/Hematite $/ \mathrm{Fe}_{2} \mathrm{O}_{3}$

8. Glass Beads

9. Cement/Concrete

10. Alumina $/ \mathrm{Al}_{2} \mathrm{O}_{3}$

11. Diatomaceous Earth

12. Toluene

13. Tetrachloroethylene

14. Mineral Oil

15. Ethylene Glycol

16. Vermiculite

17. Perlite $\left(\mathrm{SiO}_{4}\right)$

18. $\mathrm{CaSO}_{4} \cdot 2 \mathrm{H}_{2} \mathrm{O} /$ Plaster of Paris

19. Phenol

20. Fly Ash (ASTM Class F)

21. Concrete (cured, crushed, screened)

\begin{tabular}{|c|c|c|c|c|c|}
\hline 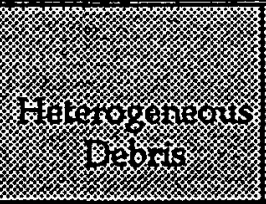 & 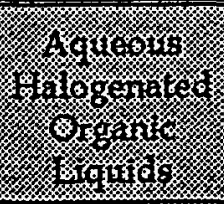 & 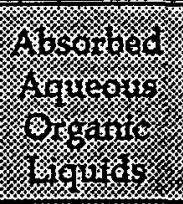 & 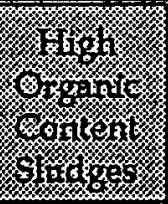 & 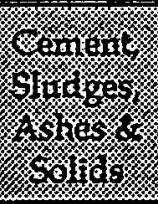 & 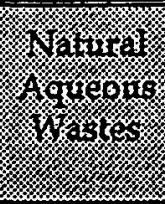 \\
\hline 5. & 5. & 5. & 10. & 10. & 5. \\
\hline 5. & 5. & 5. & 5. & 5. & 5. \\
\hline 10. & 10. & 19. & 15. & 30. & 75. \\
\hline \multicolumn{6}{|l|}{10.} \\
\hline \multicolumn{6}{|l|}{10.} \\
\hline \multicolumn{6}{|l|}{10.} \\
\hline 10. & & & 10. & 3. & \\
\hline \multicolumn{6}{|l|}{10.} \\
\hline \multicolumn{6}{|l|}{8.} \\
\hline 10. & & & 5. & 2. & \\
\hline \multicolumn{6}{|l|}{10.} \\
\hline & 10. & & & & \\
\hline . & 10. & & & & \\
\hline & 10. & & 7. & & \\
\hline & 10. & 10. & 10. & & \\
\hline & 19. & 25. & & & \\
\hline & & 25. & 10. & 10. & \\
\hline & & & 10. & 3. & \\
\hline & & & 10. & & \\
\hline & & & & 15. & 1. \\
\hline & & & & 20. & \\
\hline
\end{tabular}


TABLE 3-1:

SURROGATE FORMULATION - SUMMARY

(Cont'd)

FORMULATION NO.:

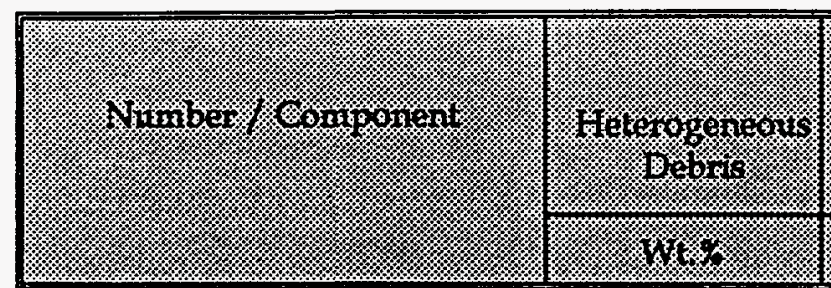

2

\begin{tabular}{|c|}
\hline 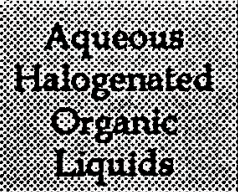 \\
\hline \\
\hline
\end{tabular}

3

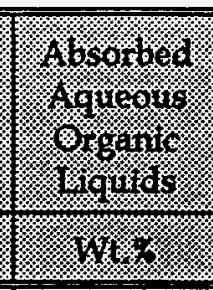

4

5

6

22. $\mathrm{CaCl}_{2}$

23. $\mathrm{NaHCO} / \mathrm{NaNO}_{3}$ *

24. $\mathrm{MgSO}_{4} \bullet 7 \mathrm{H}_{2} \mathrm{O} / \mathrm{Al}\left(\mathrm{NO}_{3}\right)_{3}$ *

25. $\mathrm{Na}_{2} \mathrm{HPO}_{4} \bullet 7 \mathrm{H}_{2} \mathrm{O}$

RCRA Metals

26. $\mathrm{Cr}\left(\mathrm{NO}_{3}\right)_{2} \cdot 6 \mathrm{H}_{2} \mathrm{O}$ (or oxide)

27. $\mathrm{Ni}\left(\mathrm{NO}_{3}\right)_{2} \bullet 6 \mathrm{H}_{2} \mathrm{O}$

28. $\mathrm{Pb}\left(\mathrm{NO}_{3}\right)_{2}$

29. $\mathrm{Cd}\left(\mathrm{NO}_{3}\right)_{2} \bullet 4 \mathrm{H}_{2} \mathrm{O}$ RCRA Onganics

30. Naphthalene $\left(\mathrm{C}_{10} \mathrm{H}_{8}\right)$

31. 1,2-Dichlorobenzene $\left(\mathrm{C}_{6} \mathrm{H}_{4} \mathrm{Cl}_{2}\right)$

Radionuclide Surrogate

32. $\mathrm{CeCl}_{3}$

33. $\mathrm{CsCl} / \mathrm{CsNO}_{3}$

|

(2)

\begin{tabular}{|l|l|}
\hline & \\
\hline &
\end{tabular}

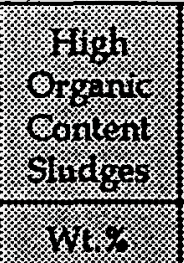

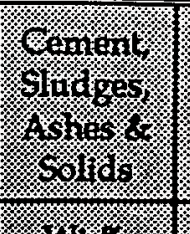

Naturat Agueonis

Wisties: 1.2.

3.

3. 3. 3. e)

\begin{tabular}{|l|}
0.1 \\
\hline 0.1
\end{tabular}

+

-

\begin{tabular}{l|l}
2 \\
\hline
\end{tabular}

\begin{tabular}{l|l}
\hline & 3. \\
\hline
\end{tabular}

\begin{tabular}{|l|l|}
\hline & 3. \\
\hline & 3. \\
\hline
\end{tabular}

* Suggested substitute for surrogate Feedstock No. 6 only. 


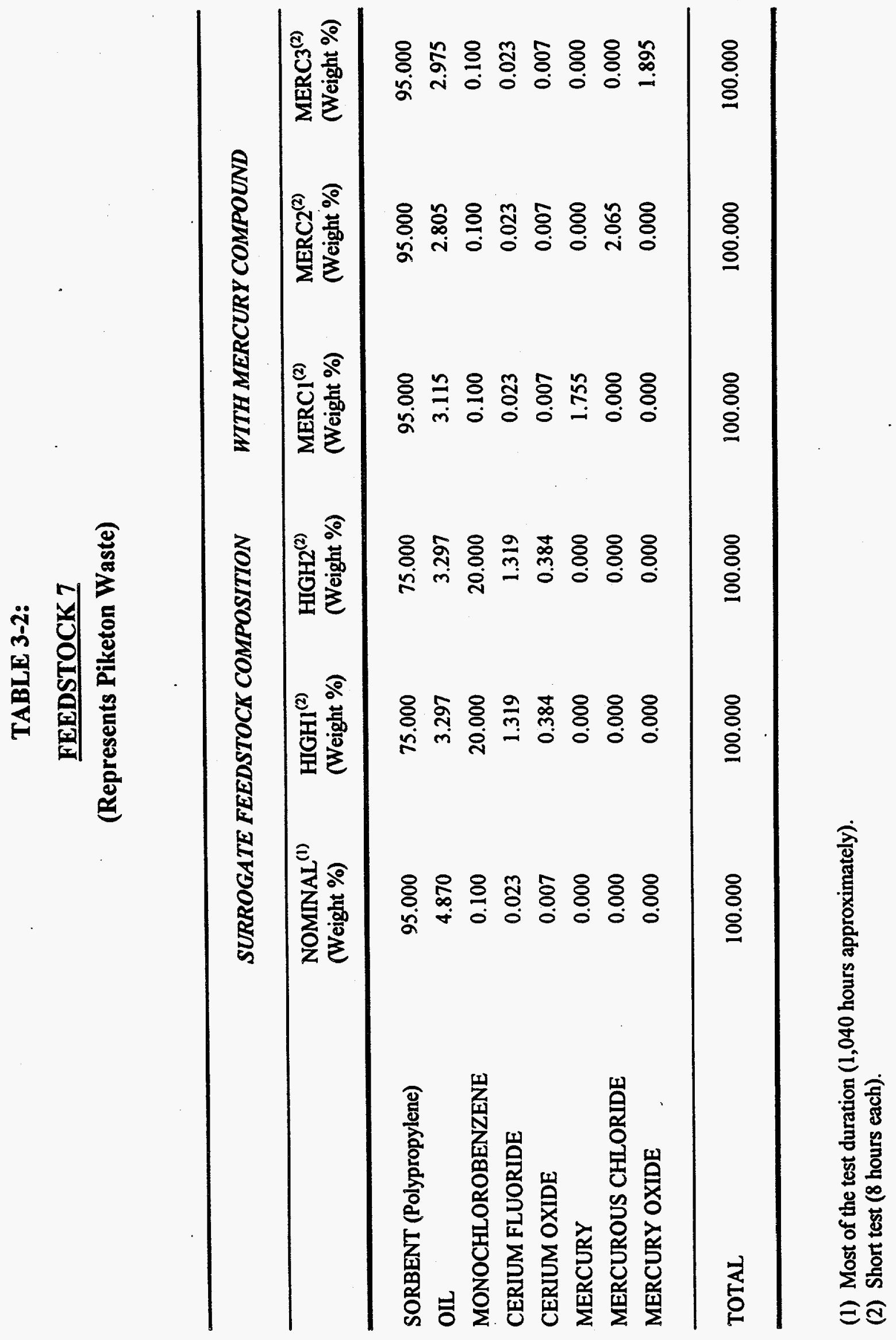


TABLE 3-3:

ULTIMATE ANALYSIS OF THE SURROGATE FEEDSTOCKS

\begin{tabular}{|c|c|c|c|c|c|c|c|}
\hline \multirow[b]{3}{*}{ COMPOSITION } & \multirow{3}{*}{$\begin{array}{r}1 \\
\mathrm{HD}\end{array}$} & \multicolumn{4}{|c|}{ FEEDSTOCK (Wt. \%) } & \multirow[b]{2}{*}{6} & \multirow[b]{2}{*}{7} \\
\hline & & 2 & 3 & 4 & 5 & & \\
\hline & & AHOL & AAOL & HOCS & CSAS & NAW & $P W$ \\
\hline Ash & 50.27 & 19.57 & 50.57 & 28.83 & 51.55 & 6.55 & 0.03 \\
\hline Moisture & 13.36 & 12.56 & 21.56 & 19.65 & 33.19 & 79.30 & 0.00 \\
\hline Carbon & 20.72 & 44.06 & 17.75 & 33.61 & 12.45 & 7.67 & 85.64 \\
\hline Hydrogen & 1.77 & 4.32 & 1.60 & 3.03 & 0.23 & 0.23 & 14.30 \\
\hline Oxygen & 3.08 & 5.24 & 5.24 & 9.73 & 0.91 & 2.49 & 0.00 \\
\hline Chlorine & 10.19 & 13.65 & 2.68 & 2.68 & 0.50 & 2.42 & 0.03 \\
\hline Sulfur & 0.58 & 0.58 & 0.58 & 2.44 & 1.14 & 0.77 & 0.00 \\
\hline Nitrogen & $\underline{0.03}$ & $\underline{0.03}$ & $\underline{0.03}$ & $\underline{0.03}$ & $\underline{0.03}$ & $\underline{0.57}$ & 0.00 \\
\hline TOTAL & 100.00 & 100.00 & $\underline{100.00}$ & 100.00 & 100.00 & 100.00 & 100.00 \\
\hline
\end{tabular}




\section{SECTION 4.0 \\ PROCESS DEVELOPMENT UNIT (PDU) \\ DESIGN AND CONSTRUCTION}

\subsection{FEED SYSTEM}

The steam reformer is continuously fed waste material using a positive displacement pump subsystem for liquids and slurries and/or a metering screw feeder with lock hoppers for solid feed material. The purpose of the feed system is to deliver the feedstock to the reactor reliably as well as accurately. Since testing involved a number of surrogate mixtures, specific equipment was required to cover the range of feedstock application.

\subsubsection{Liquid Feed System}

The liquid feed system consists of a polycarbonate carboy supply tank, calibration burette and a variable-stroke, variable-speed piston metering pump (Figure 4-1). Liquid injection is directly into the injection screw cavity leading into the reformer.

\subsubsection{Original Solid Feed System}

The project has utilized two solid feed system designs. The first solid feed system (Figure 4-2) was operated during the initial LLMW feedstock testing campaigns. The original system consisted of two separate feed hoppers, each with twin variable-speed metering screws. One hopper was designed for the surrogate waste feed, while the second hopper provided for glass frit addition. Both feed hoppers discharged into a common transition hopper. The transition hopper fed a rotary lock valve that, in turn, metered the feed into the injection screw. The injection screw conveyed the feed directly into the reactor. The screw and barrel were both cooled with once-through boiler feed water to prevent overheating and distortion of the injection equipment. 


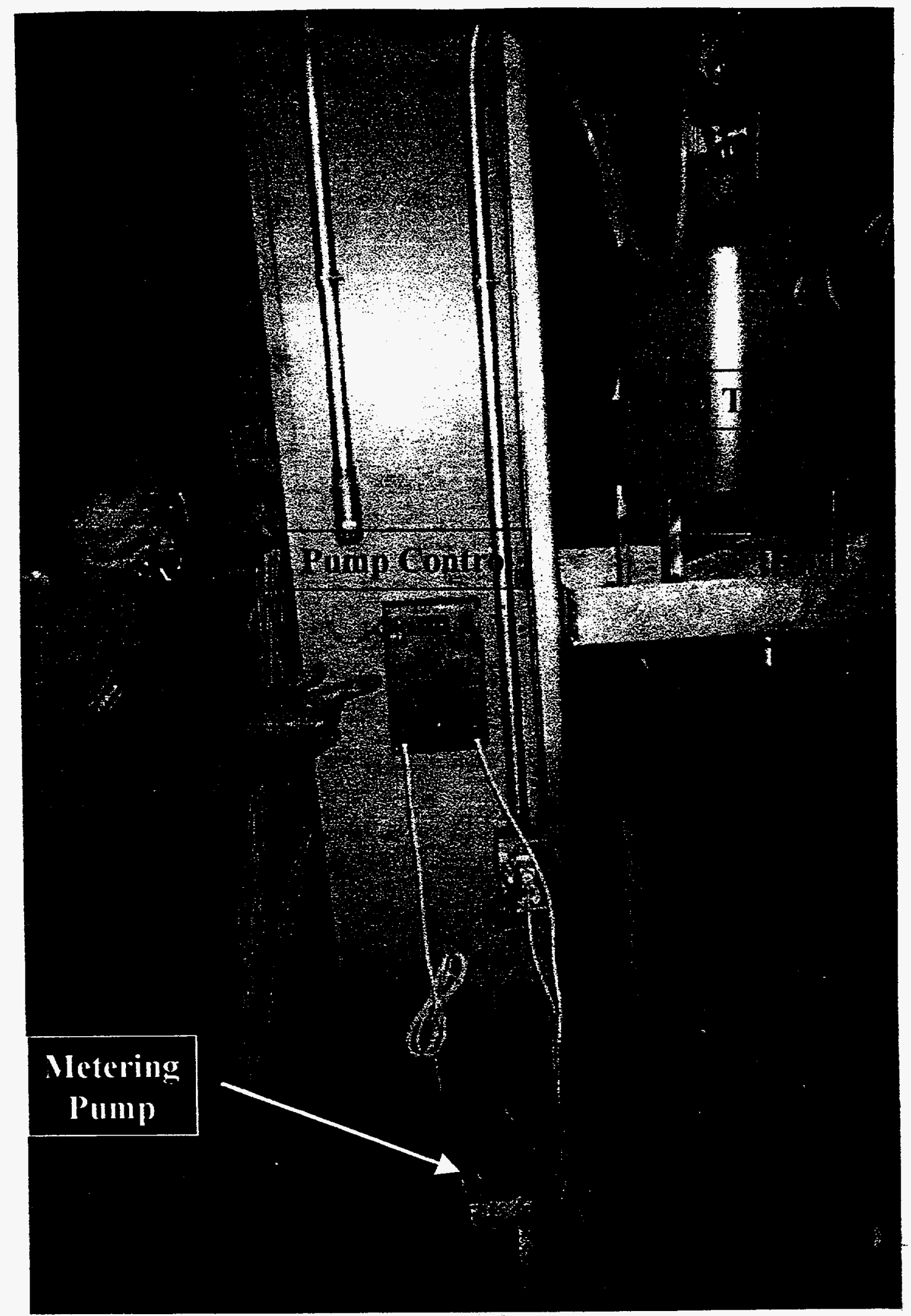

FIGURE 4-1: LIQUID FEED SYSTEM 


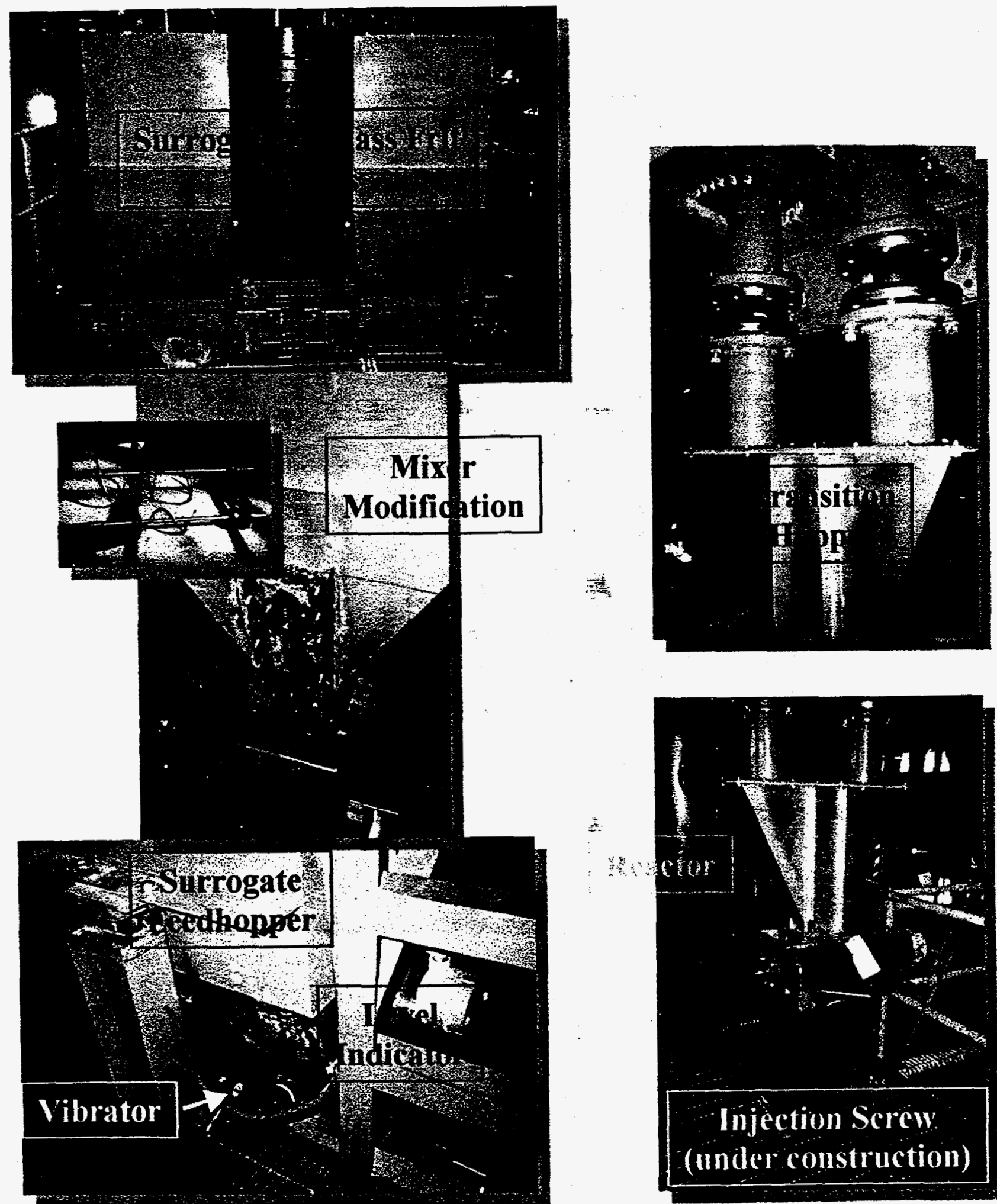

FIGURE 4-2: ORIGINAL SOLID FEED SYSTEM 
Due to bridging difficulties, the surrogate feed hopper was outfitted with a motorized mixer to dislodge the bridged material and keep the flights of the metering screws fully loaded with feedstock (Figure 4-2).

\subsubsection{New Solid Feed System (Piketon Test)}

The second solid feed system was designed and installed to handle the low-density Piketon surrogate feedstock. The two original feed hoppers were replaced with one hopper with larger variable-speed, twin-metering screws (Figure 4-3). The transition hopper and rotary lock valve were replaced with an automated lock hopper (Figure 4-4). Optical sensors detected the progress of the feed material through the system and triggered actuation of the lock hopper valves. The optical sensors also provided operator alarm in the eventuality of a plugged line.

After passing through the lock hopper, the feed charge entered the inlet of the existing injection screw. Since the lightweight Piketon feed was prone to bridging at this location, a motorized mixer was added to disperse the feed charge (Figure 4-4) as it entered the injection screw.

\subsubsection{Piketon Feedstock Shredding System}

A shredding system was utilized to prepare the oil absorbent polypropylene pads for feeding during the Piketon test (Figure 4-5). The pads were shredded and stored in supersacks prior to use.

\subsection{STEAM REFORMER}

The steam reformer is an indirectly heated fluidized bed vessel 42 inches in diameter with an active, dense bed volume of 1 square foot by 8 feet high (Figure 4-6). The vessel is constructed of mild steel pipe and is refractory-lined. The vessel is outfitted with an internal cyclone to return elutriated particles back to the bed. The fluidizing medium is superheated steam for superior mixing and heat transfer for the feed material to be treated. Electrical heaters immersed in the bed provide heat required for the reaction, as required. The PDU consist of two 


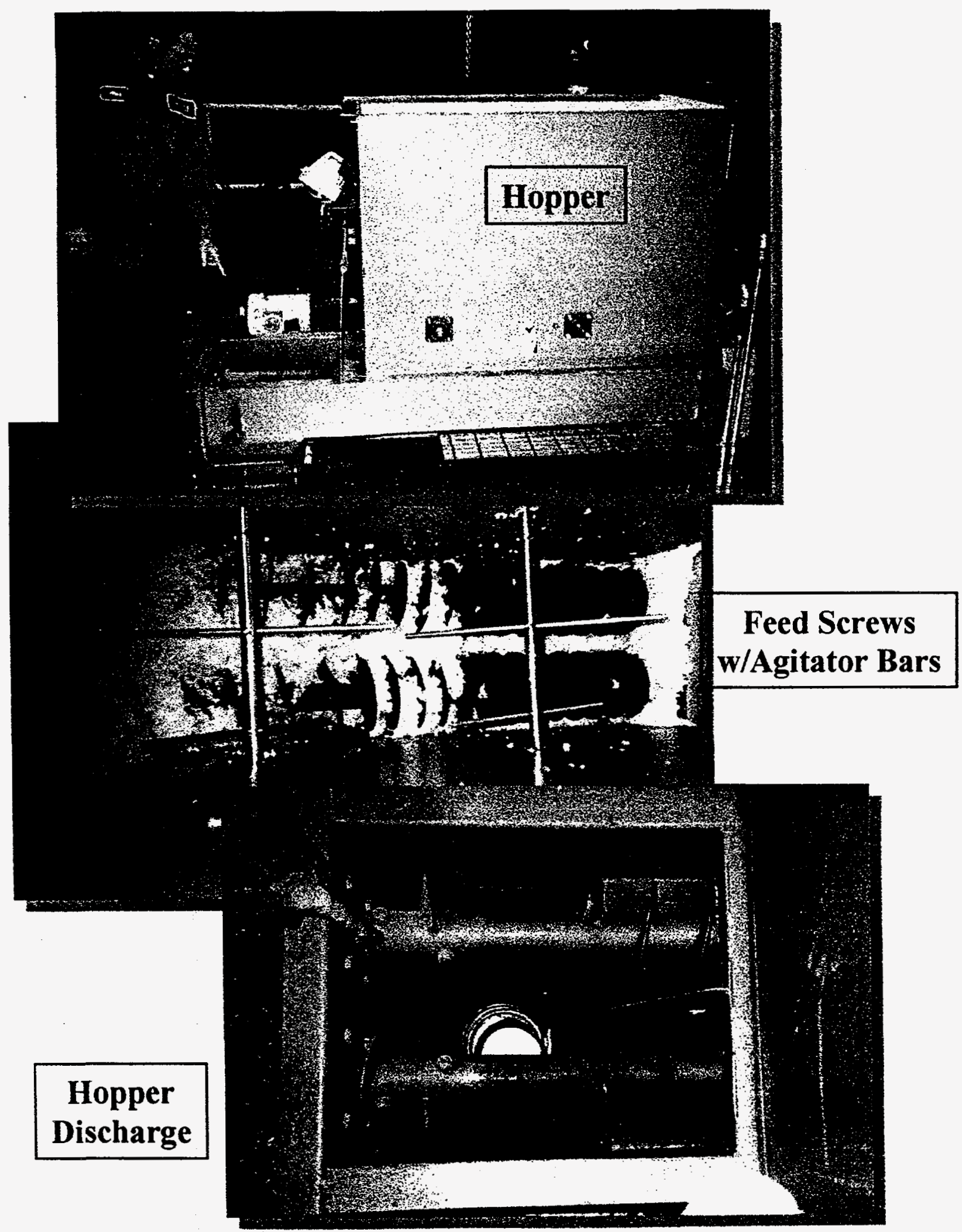

FIGURE 4-3: NEW FEED HOPPER SYSTEM 


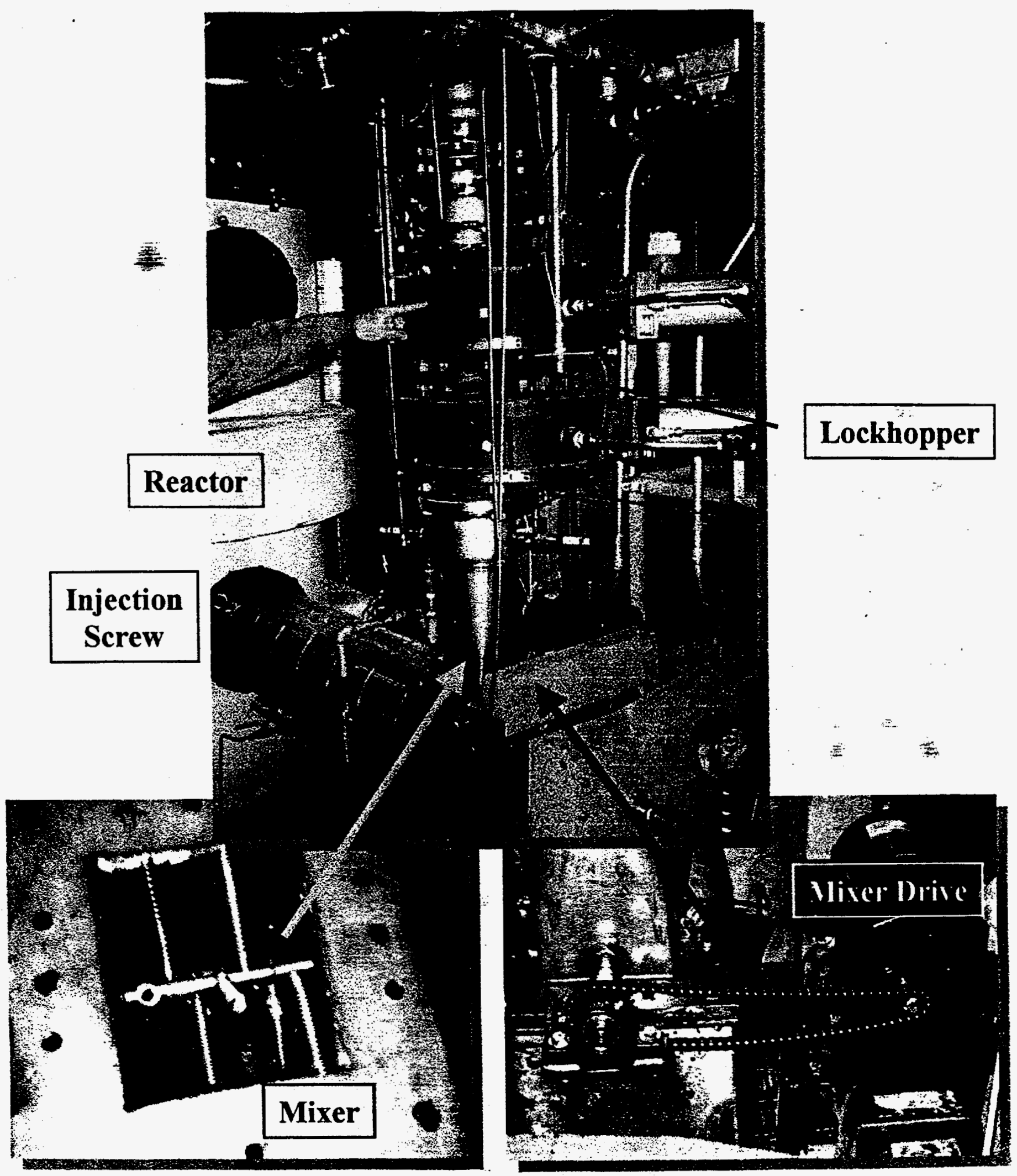

FIGURE 4-4: LOCK HOPPER AND INJECTION SCREW 


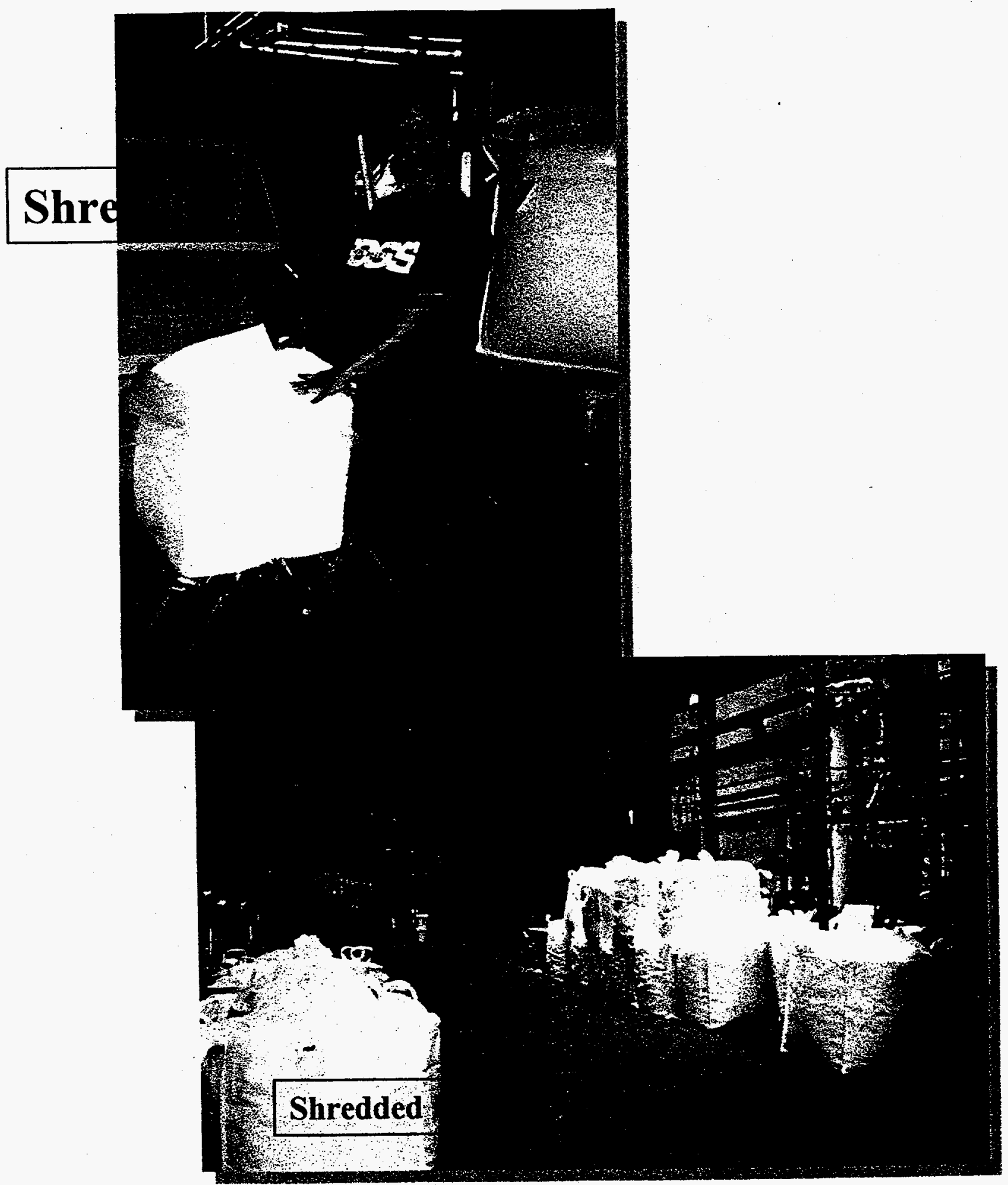

FIGURE 4-5: PIKETON FEEDSTOCK SHREDDING SYSTEM 


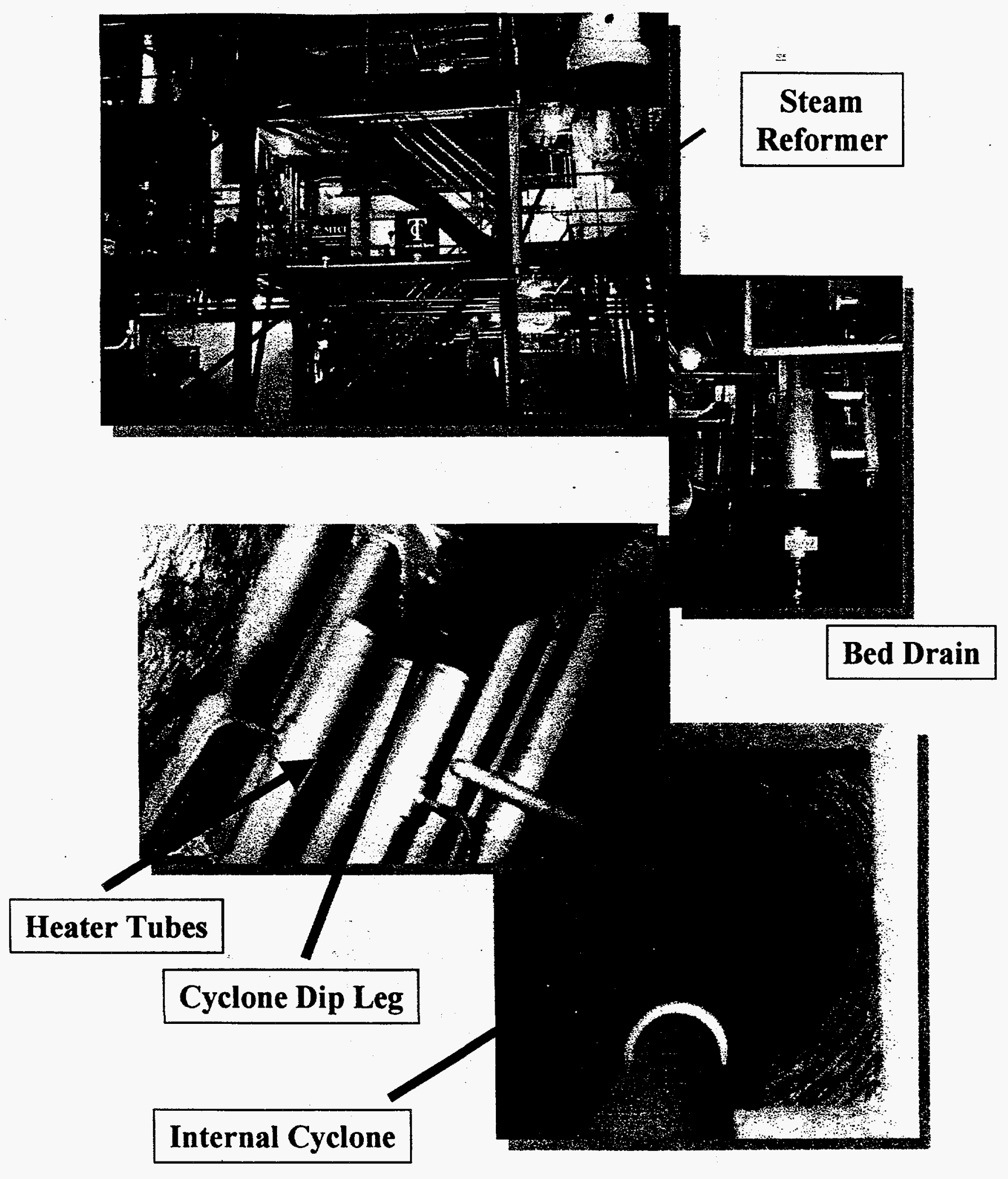

FIGURE 4-6: STEAM REFORMER 
heater modules (Figure 4-7) and provide up to 40 total $\mathrm{kW}$ of electrical heating. The bed temperature is closely controlled to ensure complete volatilization and partial steam reforming of all organic compounds and to ensure retention of radionuclides including cesium compounds in solid form along with other inorganics in the bed. If cesium is not in the feed material, the bed will be operated at $1350^{\circ} \mathrm{F}$ and essentially all of the carbon will be steam reformed, i.e., removed as synthesis gas. If cesium is present, the bed temperature will be controlled at $1050^{\circ} \mathrm{F}$ to ensure retention of cesium in the solid bed material. This temperature will result in approximately 65 percent carbon conversion with the remaining carbon exiting in solid form in the bed drain and high-temperature HEPA (HT HEPA) catch. The carbon can either be disposed of with the solid radioactive waste (volume reductions are still greater than 10 in most cases) or small oxygen can be added to the bed with the fluidizing steam in the absence of waste feed and the carbon will be converted to carbon monoxide. Even though oxygen is being added, the bed remains as a reducing environment and the temperature is closely controlled to $1050^{\circ} \mathrm{F}$ by reducing power to the electrical heaters.

\subsection{STEAM REFORMER GAS CLEANUP}

In addition to the internal cyclone, two high-temperature pulsed jet HEPA filters (Figure 4-8) serve to essentially eliminate the carryover of particulate in the synthesis gas stream. The HT HEPA filters are silicon carbide ceramic candles that operate effectively at approximately $1000^{\circ} \mathrm{F}$. The two HT HEPA filters provide redundancy and can actually be operated in series or

parallel. Several tests have shown that the filters achieved greater than 99.97 percent particulate removal for 0.3-micron particles.

\subsection{THERMATRIX FLAMELESS OXIDIZER}

The particulate-free synthesis gas from the HT HEPA filters flows into the Thermatrix flameless thermal oxidizer (FTO). The FTO is a non-incineration system that converts the hydrogen carbon monoxide and hydrocarbon vapors into water vapor, carbon dioxide, and acid gases. Screening tests in the coupled steam reformer-FTO system have consistently demonstrated 


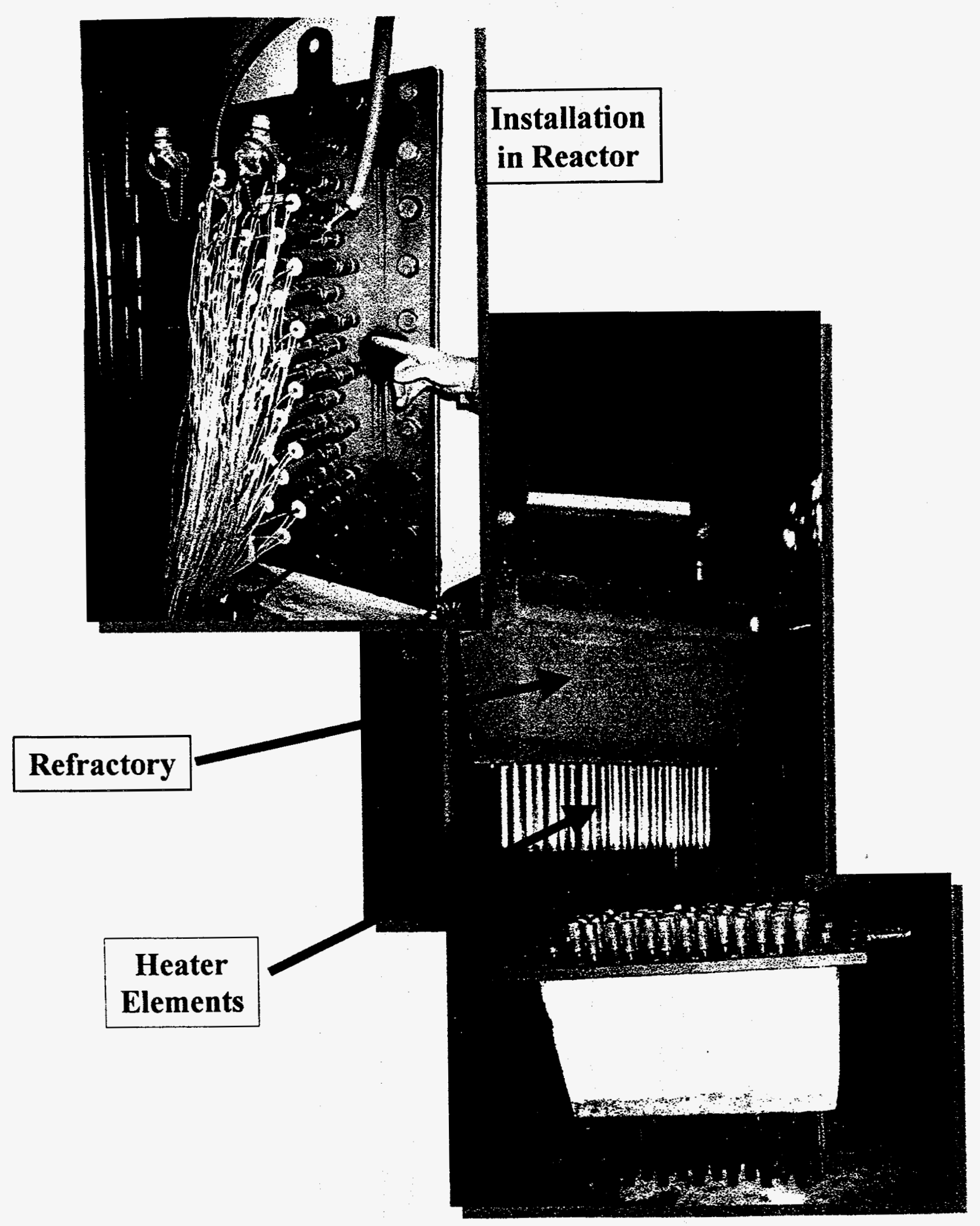

FIGURE 4-7: ELECTRIC HEATER MODULES 


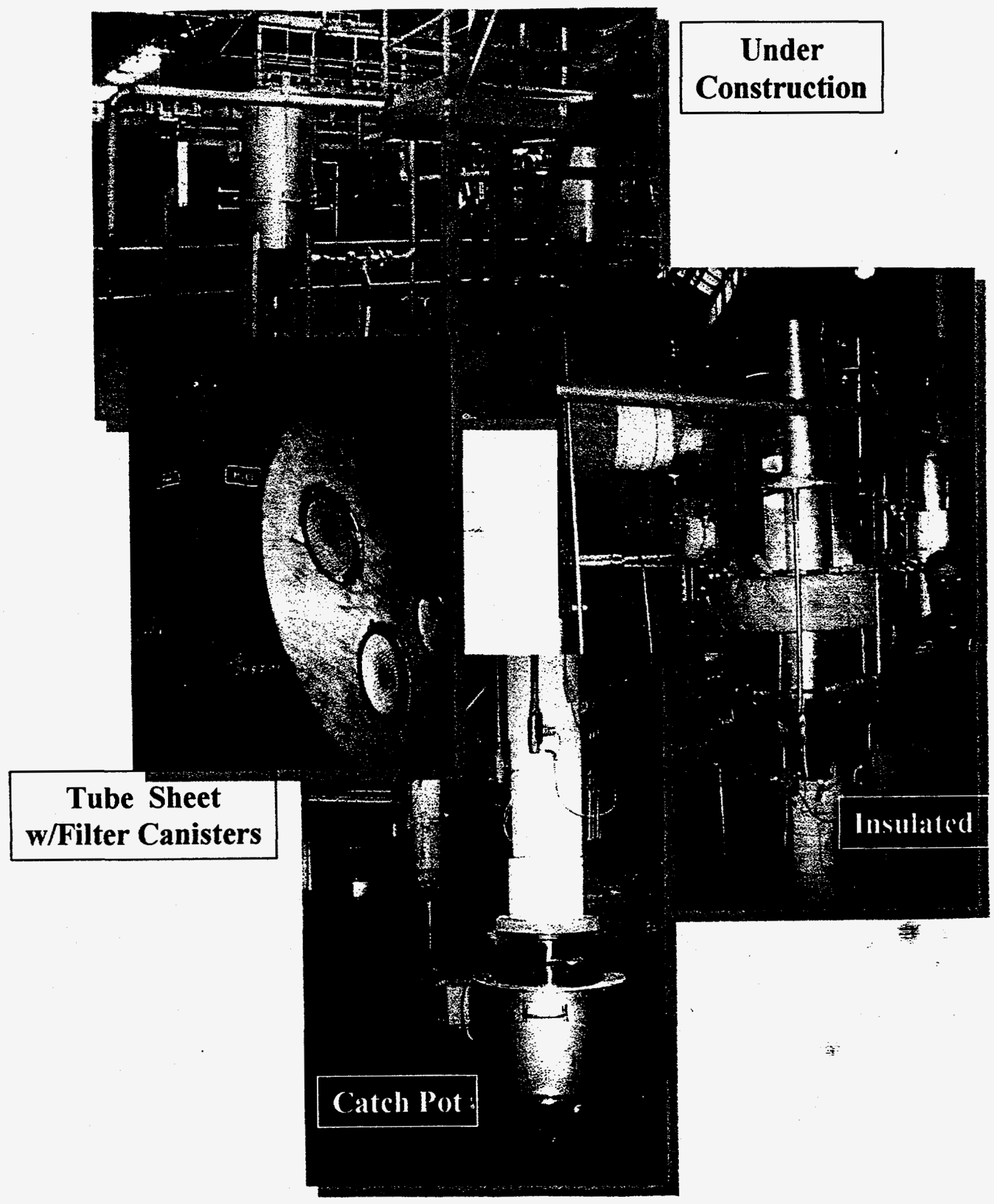

FIGURE 4-8: HEPA FILTERS 
Destruction and Removal Efficiency (DRE) of $99.99+\%$ for Principal Organic Hazardous Constituents (POHC) and even $99.99999 \%$ DRE for PCB surrogate.

The oxidizer (Figure 4-9) is insulated vessel packed with ceramic material according to a proprietary matrix configuration. At start-up, the ceramic matrix is preheated to between $1600^{\circ} \mathrm{F}$ and $1800^{\circ} \mathrm{F}$. Once the preheating has been completed, the synthesis gas from the steam reformer containing carbon monoxide, unconverted hydrocarbons, hydrogen and steam is premixed with excess air and the oxygen-rich $800^{\circ} \mathrm{F}$ mixture is introduced into the oxidizer. The fume stream first passes through a mixing zone and then through the reaction zone of preheated ceramic material where complete flameless oxidation occurs in a fraction of a second. The oxidation process releases heat that is absorbed and conserved by the ceramic matrix which helps maintain consistent oxidation temperatures between $1600^{\circ}$ and $1800^{\circ} \mathrm{F}$. The expansion of the gases as they heat up is accommodated by an inerease in the ceramic matrix void volume. Due to its flameless design and consistent temperature profile, the oxidizer achieves very high DRE on gas streams with heating values as low as $10 \mathrm{Btu} / \mathrm{scf}$ with de minimis $\mathrm{NO}_{\mathrm{x}}$ formation $(2 \mathrm{ppm})$. The Thermatrix system also has no diogin emissions because of excess oxygen (at least $8 \%$, more commonly $11 \%$ ), greater than $1600^{\circ} \mathrm{F}$, no possible bypassing of the reaction zone, and elimination of cold surfaces in the exhaust flow path. The matrix oxidizer is exceptionally safe and is even certified for use in highly flammable environments where conventional systems are prohibited.

\subsection{THERMATRIX GAS CLEANUP}

The exhaust of the flameless thermal oxidizer (FTO) may contain sulfur dioxide and hydrochloric acid from sulfur and chlorine compounds originally in the waste stream. The exhaust gas from the FTO is connected to a gas-cleaning system (Figure 4-10) which removes acid gases as dry salts. The gas-cleaning system includes a spray dryer for gas cooling, partial acid gas removal and salt drying followed by a medium-temperature $\left(350^{\circ} \mathrm{F}\right)$ pulsed jet baghouse provides salt recovery. A low-temperature HEPA (LT HEPA) filter provides for final particulate and radionuclide control and a packed bed scrubber with integral mist eliminator provides tail-end acid gas removal. Finally, tandem-activated carbon beds provide for mercury control prior to entering a high-pressure vacuum blower for system draft control which exhausts to the stack. 


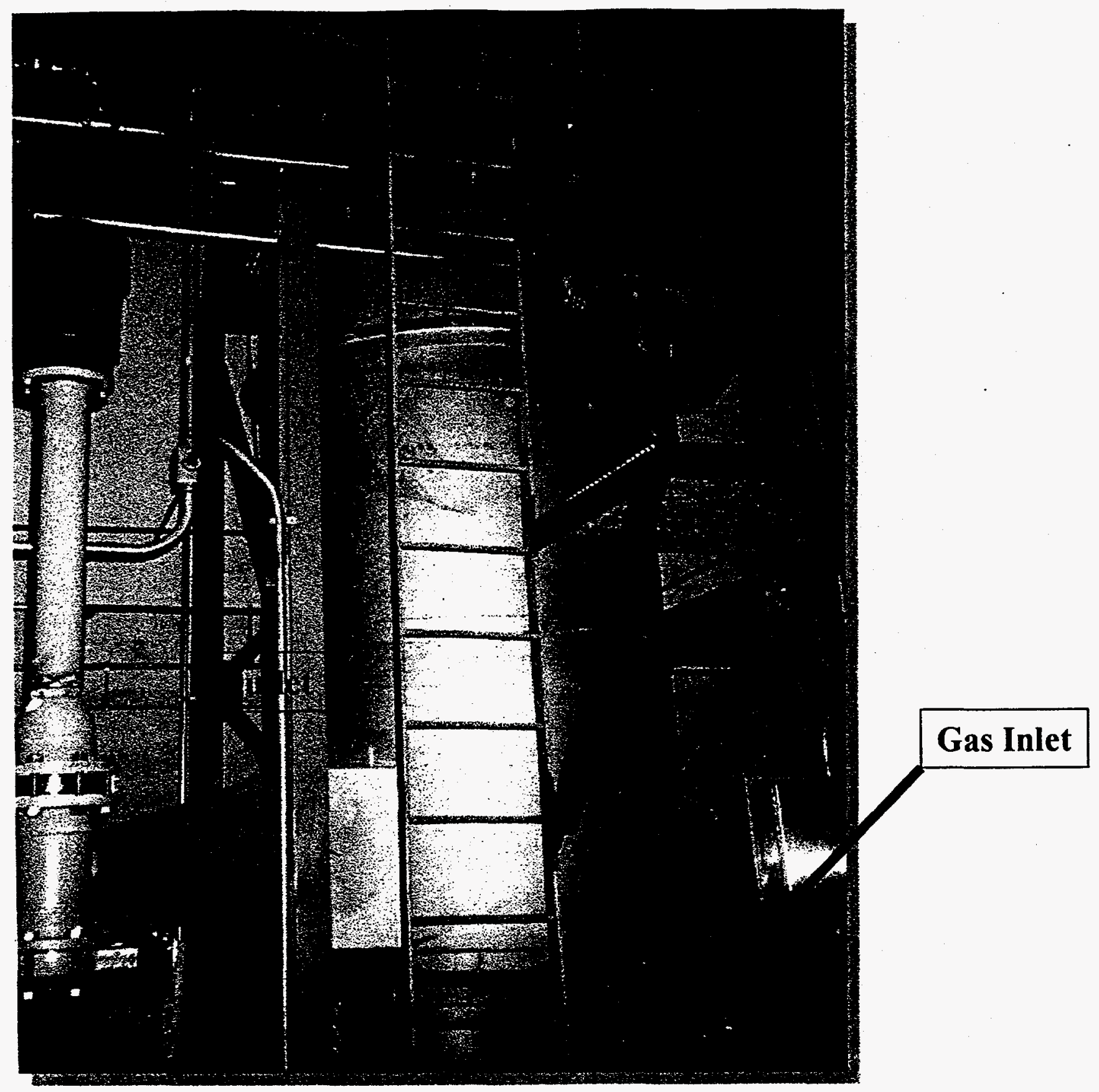

FIGURE 4-9: FLAMELESS OXIDIZER 

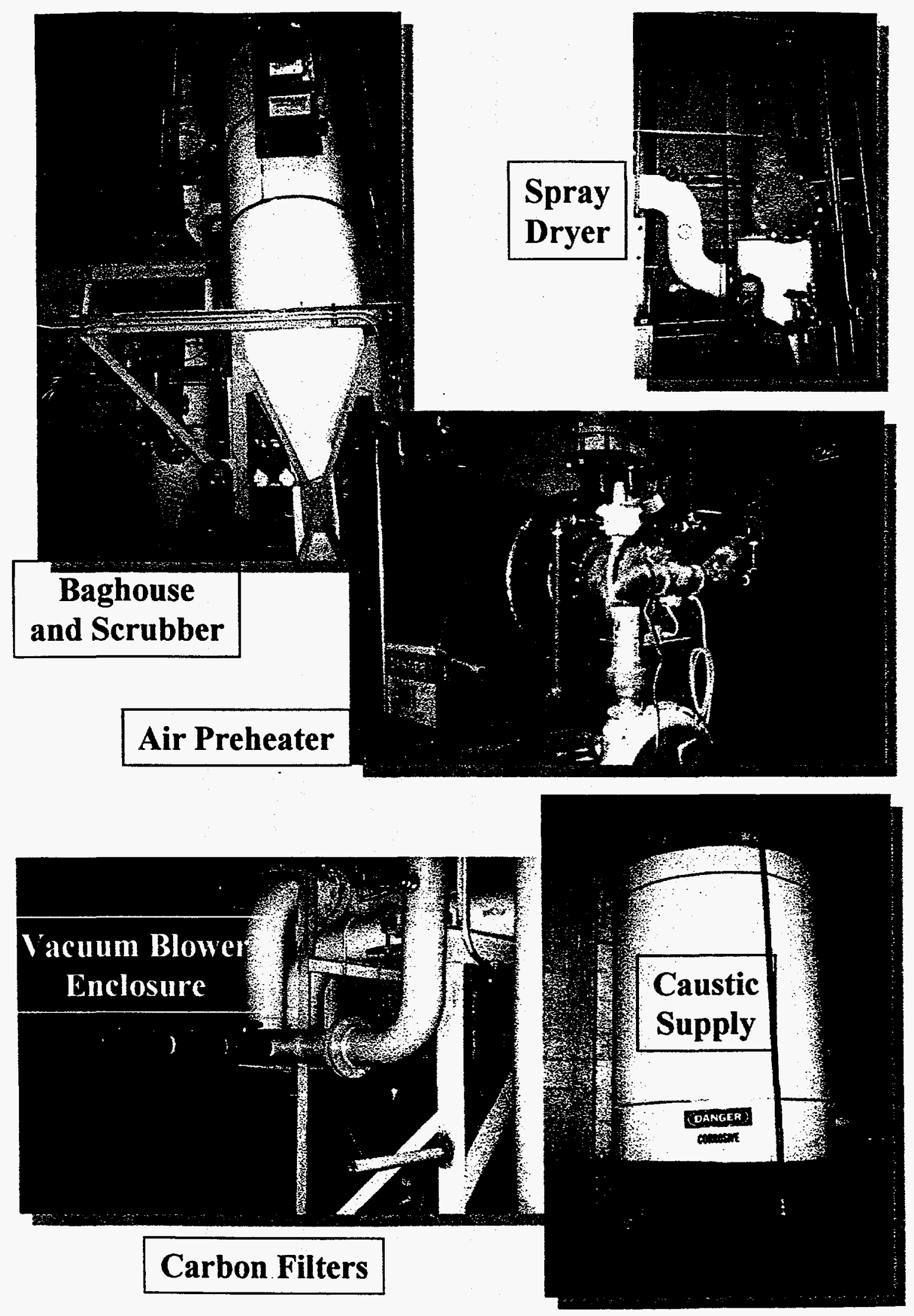

FIGURE 4-10: FLAMELESS OXIDIZER BoP 


\subsection{STEAM SYSTEM}

\subsubsection{Boiler}

A natural gas-fired boiler provides the supply of saturated steam necessary for operating the plant (Figure 4-11). Boiler feed water is supplied by a reverse osmosis (RO) water treatment system with supply tank and redundant boiler feed water pumps.

\subsubsection{Superheater}

Superheat supply steam to the steam reformer is supplied by two stages of superheating, first electric followed by gas-fired (Figure 4-12). 


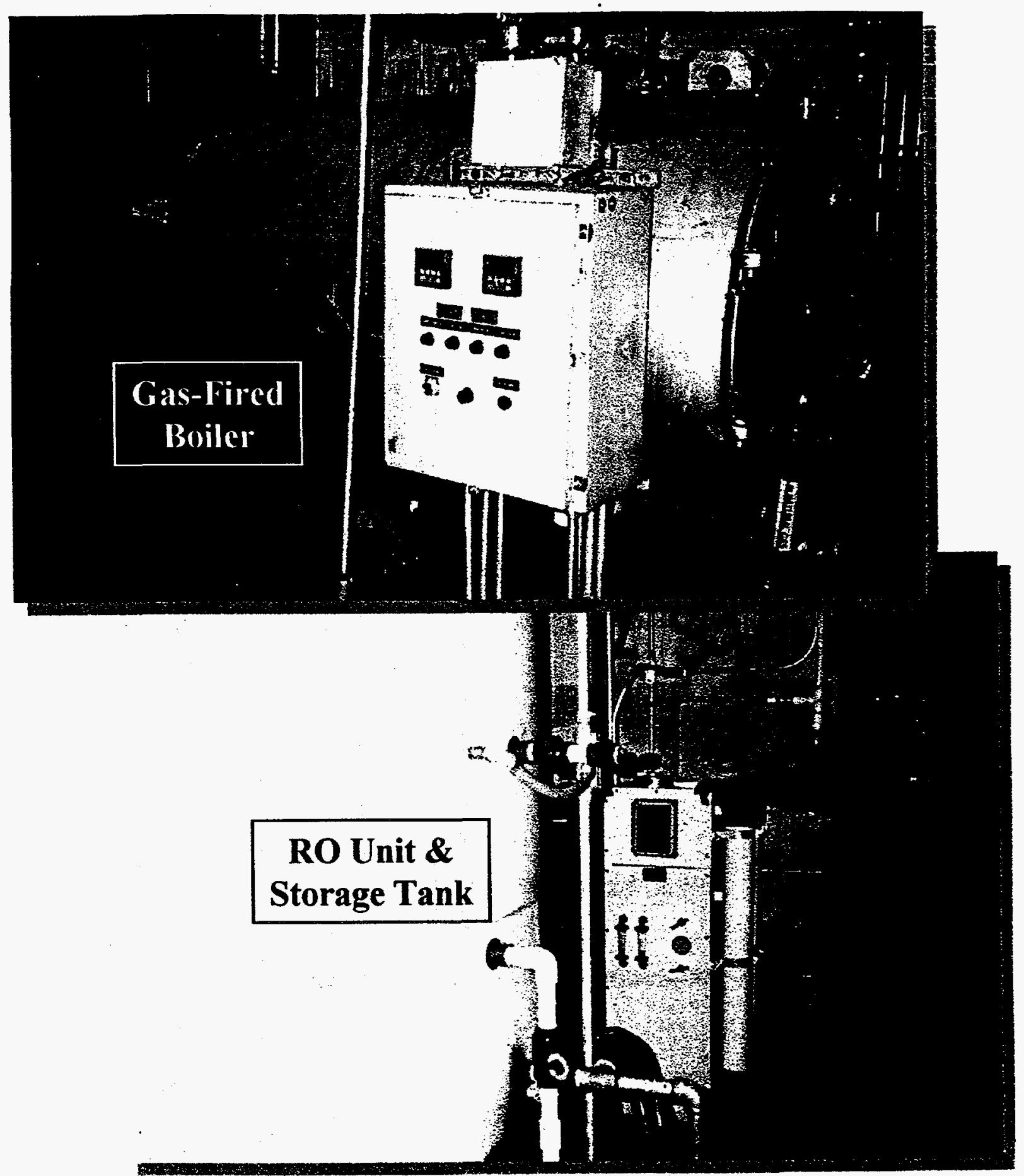

FIGURE 4-11: SATURATED STEAM BOILER AND FEED WATER SYSTEM 


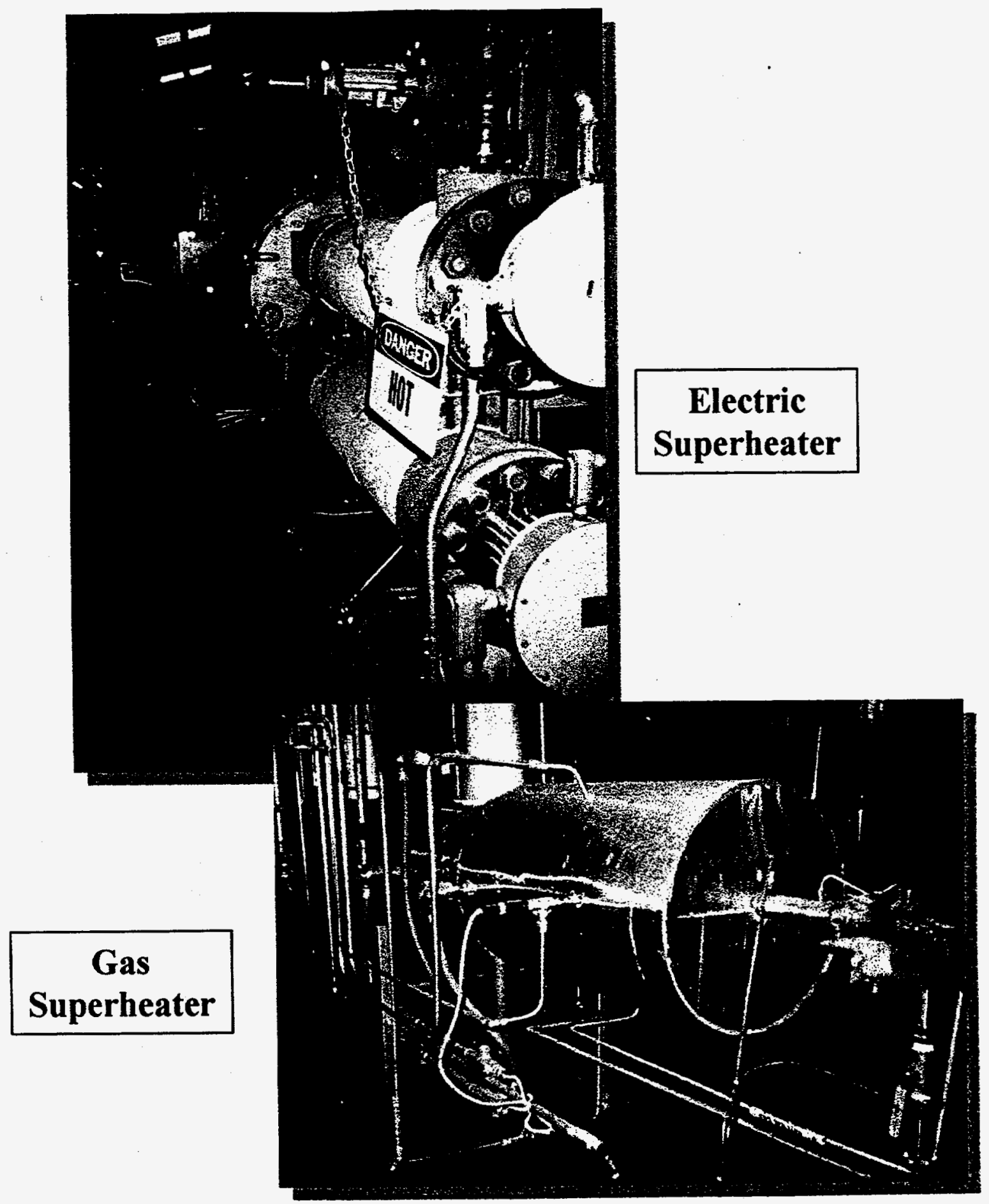

FIGURE 4-12: STEAM SUPERHEATERS 


\section{SECTION 5.0}

\section{TEST PLAN}

A detailed Test Plan was prepared and submitted to the DOE. This is included as Appendix $B$. The Test Plan includes test objectives, test matrices and test metrics. Apart from a discussion of preliminary process flow simulation, the Test Plan includes descriptions of start-up, operation and shutdown procedures, calibration procedures, sampling and analysis protocols, error assessment, emergency shutdown procedures and residual waste disposal. 


\section{SECTION 6.0 \\ TESTS AND RESULTS}

\subsection{TEST OBJECTIVES}

To evaluate performance and operability of the system. Specifically, the targets were:

- Destruction and Removal Efficiency (DRE) for RCRA Organics and POHC

- Radionuclide retention

- Volume/mass reduction

- Material balance

- Energy balance

- Carbon conversion

- $\mathrm{HCl}$ and $\mathrm{S}$ capture

- Metals retention

- Stack emissions

- Steam reformer product gas composition

- Safe disposability of solids

- Continuous and extended duration operability

\subsection{TEST MATRIX}

Four different test campaigns were conducted as follows:

- Preliminary screening test in a small-scale steam reformer test unit,

- Multiple surrogate feedstock tests in the Process Development Unit (PDU),

- Piketon surrogate waste tests in the PDU, and

- Multiple organic feedstock retests in the PDU.

The preliminary screening test was performed on August 6, 1996. The feedstock was ion exchange resins. The multiple surrogate feedstock testing occurred in three stages: Stage 1 commenced on February 8, 1997 and concluded on February 14, 1997. The feedstock was natural aqueous waste (No. 6). Stage 2 testing occurred from April 17, 1997 to April 21, 1997. 
Three feedstocks were tested viz. Nos. 2, 4 and 3 (see Table 3-1). Stage 3 started on May 20, 1997 and ended on May 21, 1997. The feedstock was heterogeneous debris (No. 1). For the multiple surrogate feedstock tests, the PDU on-line time totaled 248 hours with a feed/test time of 106 hours.

The Piketon surrogate waste testing occurred in two stages: Stage 1 started on August 15, 1997 and ended on August 29, 1997. Stage 2 commenced on September 21, 1997 and concluded on October 10,1997. The operating time for the Piketon surrogate waste test totaled 750 hours.

The multiple organic feedstock retests were performed during the period between December 2 and December 12, 1997. Three tests were performed with three different organic formulations.

\subsection{PRELIMINARY SCREENING TEST}

In preparation for extensive tests of surrogate materials in the PDU, a screening test was performed in a smaller steam reformer test unit with ion exchange resins to acquire operating experience and collect preliminary data on performance. It is to be noted that ion exchange resins are a part of each of the six surrogate feedstocks (see Table 3-1). Specifically, the objective of this test was to determine how well the surrogate cesium is retained in the bed solids and to determine the concentration of surrogate cesium in the synthesis gas stream, if any.

Figure 6-I depicts the test unit used for the screening test. This is essentially a modified version of the unit used previously in California for the initial tests under this contract. The system consists of a pulse combustor with two $U$-shaped resonance tubes (fluidized bed heat source), superheater (fluidizing steam source), fluidized bed reactor, air-tight screw feeder, two cyclones, flare, venturi scrubber, ID fan and stack. The pulse combustor resonance tubes supply heat to the reactor and the flue gas is used for heat exchange in the superheater. The resin dries, devolatilizes, and undergoes steam-reforming reactions in the fluidized bed. The pulse combustor 


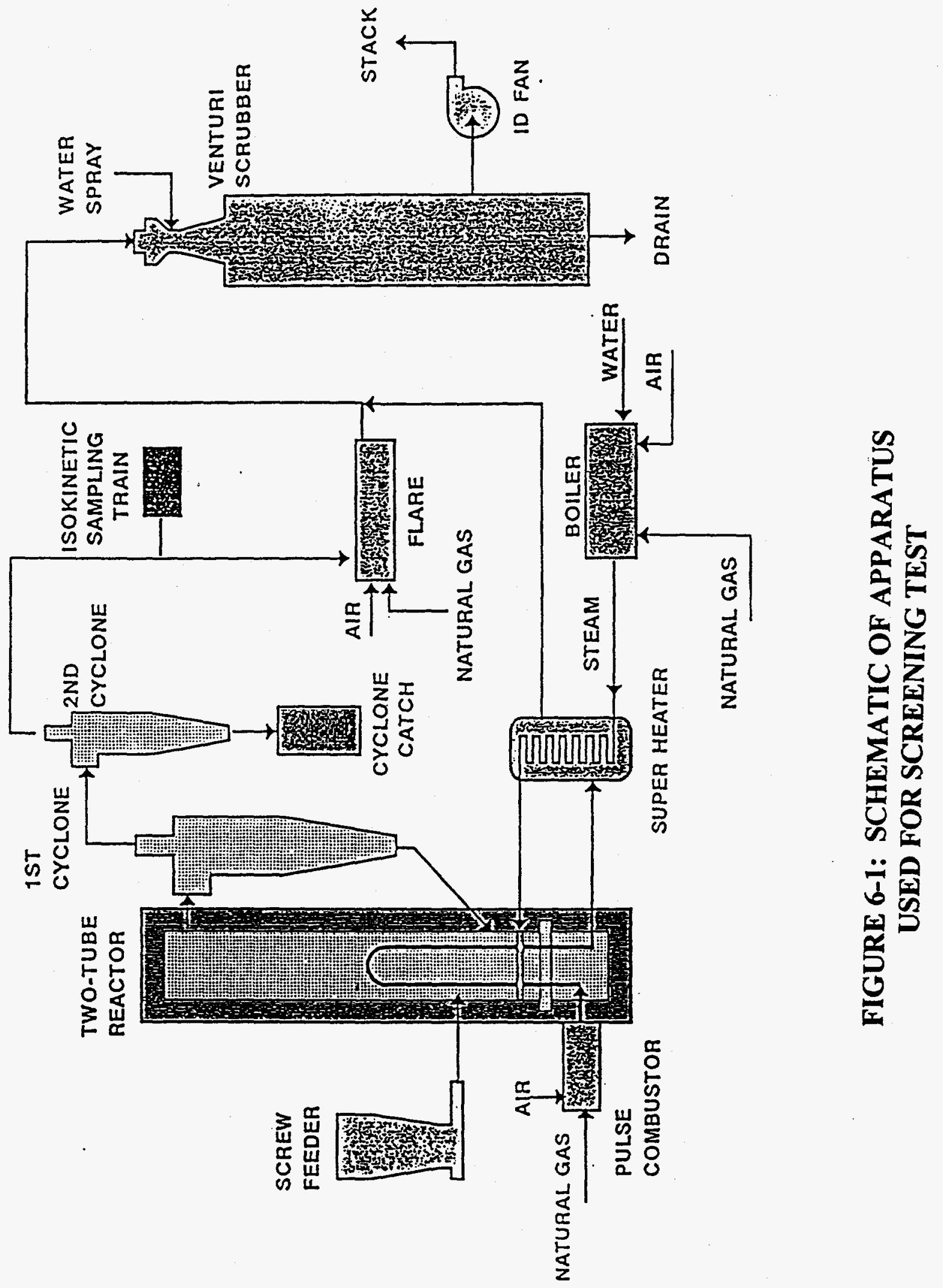


supplies the endothermic heat of reaction and its firing rate can be adjusted to operate the fluidized bed in the temperature range between 1000 and $1600^{\circ} \mathrm{F}$. The reformate gases (rich in hydrogen) pass from the reactor through two cyclones to the flare where they are burned in the presence of a natural-gas burner flame. Gases exiting the flare and superheater pass through the venturi scrubber and are conveyed to the stack by the $\mathrm{D}$ fan.

Several short shakedown tests were performed on the system with and without resin feed to verify operability. Two 55-gallon drums of spent utility ion exchange resin and $1.5 \mathrm{~kg}$ of cesium chloride were acquired for the screening test. To prepare surrogate waste, cesium chloride $(\mathrm{CsCl})$ was thoroughly dissolved in distilled water and the solution was mixed with resin. The mixture stayed in the MTCI laboratory for 24 hours for $\mathrm{CsCl}$ adsorption by resin. The water was drained afterwards and the $\mathrm{CsCl}$-doped resin was spread on a large flat surface for air drying. Analysis of the resin is presented in Table 6-1.

TABLE 6-1:

\section{ANALYSIS OF RESIN}

\begin{tabular}{lr} 
Composition: & Wt.\% \\
\hline Moisture & 13.99 \\
Ash & 1.72 \\
Carbon & 50.60 \\
Sulfur & 5.93 \\
Hydrogen & 5.69 \\
Nitrogen & 4.64 \\
Oxygen & 17.28 \\
Cesium & 0.17
\end{tabular}

At the beginning of the test, 131 pounds of silica sand ( 200 to 300 microns size) were loaded into the reactor. The reactor temperatures and pressures were monitored to verify stable operation. Sixty pounds of doped resin were fed to the steam reformer over a period of approximately five hours. The mean bed temperature during the test was controlled to a set point of $1050^{\circ} \mathrm{F}$. In order to determine surrogate cesium carryover, an isokinetic sampling probe was 
located in the straight pipe downstream of the second cyclone. The gas sample passed through a 5 micron pore size sintered metal filter and a sample train that included a 12-inch high column of distilled water, a rotameter and an eductor. The metal filter was heat traced with a $500^{\circ} \mathrm{F}$ electrical heating tape to prevent condensation and the water column was cooled externally by ice-water mixture to aid condensation. Two gas samples were taken during the test at 20-minute intervals. A total isokinetic sampling time of 35 minutes was used to collect solids in the sintered metal filter for analysis. Gas samples were collected in tedlar bags as well by drawing samples downstream of the waste column using a vacuum pump.

Following is the full list of samples collected and sent for analyses:

- $\quad$ Pure resin, ultimate analysis;

- $\quad \mathrm{CsCl}$ doped resin, analysis for $\mathrm{Cs}$ content and moisture content;

- Bed drain sample, analysis for Cs content and ultimate analysis;

- Cyclone catch sample, analysis for Cs content and LOI analysis;

- Solids collected in the sintered metal filter, analysis for Cs and other metals content;

- Condensed water sample, analysis for Cs and other metals content; and

- Two gas samples taken downstream of water bath, analysis for Cs content.

A total of 0.1248 pound of cesium was fed and a total of 0.1152 pound was collected, yielding 92.3 percent cesium mass balance. This is short of the 100 percent desired and is due to solids left undrained in the first cyclone dipleg. The cesium concentration in the gas samples collected from the sampling train was below the detection limit of 1 part per billion. By assigning an average value of $0.5 \mathrm{ppb}$ to this sample, the cesium escaping with the product gas stream was estimated. The cesium entrainment in gas was calculated to be 0.000025 percent and the cesium retention in the steam reformer was evaluated to be 99.999975 percent.

A total of 38.012 pounds of carbon were fed and a total of 12.904 pounds of carbon were collected in the solids, yielding a carbon conversion of 66.1 percent. This is consistent with data for steam reforming of various feedstocks at low temperatures. For example, the carbon conversion for steam reforming black liquor at $1065^{\circ} \mathrm{F}$ in a 50 tons/day system turns out to be on the 
order of 60 percent. Finally, for resin treatment, these results translate into a mass reduction exceeding 10 to 1 .

In summary, the screening test was successful and pointed out that (i) cesium can essentially be retained in the solids (as a carbonate), (ii) organics, sulfur and chlorine can be separated from the solids stream, and (iii) significant volume and mass reduction can be achieved.

\subsection{PDU TESTS}

Multiple surrogate feedstocks (Nos. 6, 2, 4, 3 and 1 - see Table 3-1) were tested in the PDU unit to evaluate performance and operability. The Piketon surrogate waste (see Table 3-2) was also tested in this unit to verify performance and extended term operability. Three different organic formulations were also tested in this unit to verify $99.99+\%$ DRE (see Table 6-2). These tests were all conducted during the period from February to December 1997.

\subsubsection{Test Description}

Feedstock Preparation. Feedstock raw materials were purchased from known suppliers and kept in unopened containers until the feedstock was to be made up. Before the start of each test, the required quantity of test specimen was prepared according to the mixing instructions included in Appendix A. The prepared feedstock was kept in a closed 55-gallon drum in a cool area and out of direct sunlight.

PDU Preparation. The solid and liquid feed systems, the temperature and pressure transmitters and the flow meters were all calibrated. The controls and data acquisition system (consisting of ICOM user interface for Allen Bradley PLC, Wintelligent software, and two Pentium computers) was checked out. Test data sheets were prepared. Start-up and operational procedures were also delineated. These included the following: 

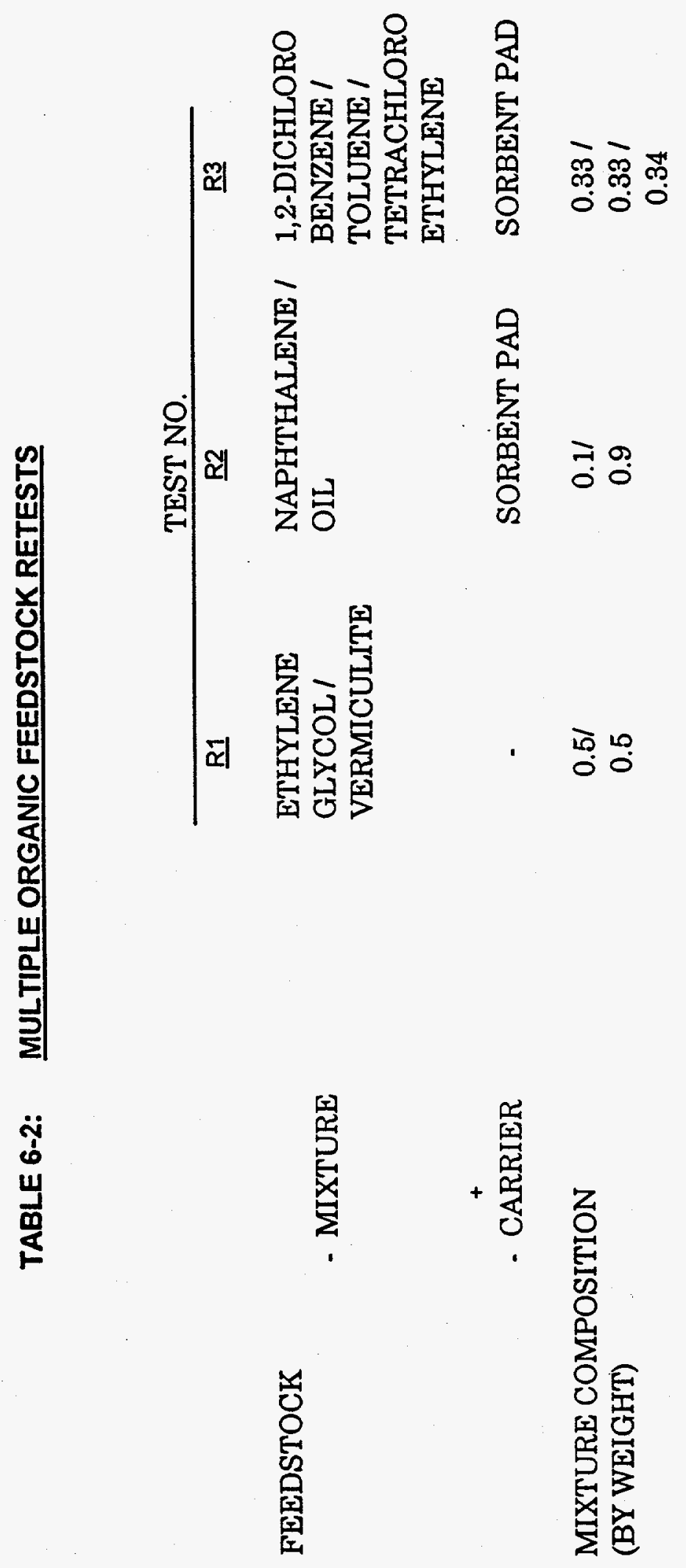
A mechanical checkout was to be first performed to assure that all components were in working order and that a sufficient supply of compressed air and nitrogen purge gas was available to perform the test. The unit was to be empty of sand or bed material.

The start-up of the PDU consisted of the following steps:

1) Start air compressor.

2) Start boiler. When water starts to boil, start superheater. Steam should be vented at this step.

3) Start the vacuum blower.

4) Supply fluidizing air to pass through the system, including the first-stage steam reformer, HT HEPA filters, thermal oxidizer, spray dryer, baghouse, LT HEPA filter, activated carbon filter, vacuum blower and stack.

5) Start feeding the bed material (sand).

6) Turn on electric heater of the first-stage steam reformer and raise the temperature such that the thermocouple embedded in the refractory increases at the rate of $100^{\circ} \mathrm{F}$ per hour. When the temperature reaches the operating window of 1000 $1200^{\circ} \mathrm{F}$, the thermostat will maintain the desired bed temperature in the range of 1000 to $1200^{\circ} \mathrm{F}$.

7) Turn on air to the thermal oxidizer. This will raise temperature of the thermal oxidizer. Maintain the temperature increases at a rate of $100^{\circ} \mathrm{F}$ per hour. When the temperature exceeds $1950^{\circ} \mathrm{F}$, manually reduce the gas supply to maintain the temperature at about $1950^{\circ} \mathrm{F}$. Supply a small amount of superheated steam, if necessary, to the first-stage steam reformer to regulate the temperatures in all the zones.

8) Monitor the temperature at the exit of the spray cooler. When the temperature reaches $250^{\circ} \mathrm{F}$, turn on the water pump of the soda ash/water feed system. The agitator tank should be filled by water (no soda ash) at this time.

9) When the temperature at the inlet to the LT HEPA filter reaches $250^{\circ} \mathrm{F}$, switch fluidizing medium from compressed air to superheated steam.

10) Maintain the overall system temperature profile during steam fluidization at design values for one hour. 
This marks the end of preheating and signals the readiness for surrogate feeding.

Testing. Typically, the PDU was started up and allowed to warm-up gradually. At the conclusion of the preheating period, the feed was started. Based on a first order analysis of theoretical bed concentration profile, between 24 and 48 hours of preconditioning with feed was determined to be desirable to load the bed with carbon and ash. Due to the high cost of RCRA organics and surrogate radionuclides, this preconditioning was performed with a feedstock similar to the test surrogate but without RCRA organics and radionuclides. After preconditioning, samples of bed, HT HEPA 1 and HT HEPA 2 catch and baghouse catch were collected. Then the actual surrogate feed was started and maintained for 6 to 8 hours. The typical test conditions during the test campaigns were:

Waste feed rate, $\mathrm{lb} / \mathrm{hr}$ Steam feed rate, $\mathrm{lb} / \mathrm{hr}$ Bed temperature, ${ }^{\circ} \mathrm{F}$

\section{Multiple Surrogates}

20-70

$75-110$

$1,035-1,055$

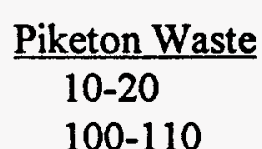

$1,200-1,215$
Multiple Organics

$15-60$

$90-95$

$1,050-1,055$

Table 6-3 provides a summary of the operating conditions for the multiple surrogate tests and Tables 6-4 and 6-5 provide similar data for the Piketon waste tests and the multiple organic feedstock retests, respectively. While the multiple surrogate tests correspond to discrete, limited duration tests with different feedstocks, the Piketon waste test was a continuous run with discrete samples taken at different time intervals. The organic retests were discrete, limited duration tests with different organic formulations.

System Process Parameters. During the actual surrogate feedstock processing, all process parameters (pressure, temperature, flow rate, and bed level) were monitored, acquired and stored by the ICOM-Wintelligent-PC data logging and management system.

Sampling. At the start of the test, initial representative samples of surrogate waste, bed material, caustic and scrubber solution were taken. At the end of the test, final representative samples of these streams were taken. These along with the different catch material were used to 


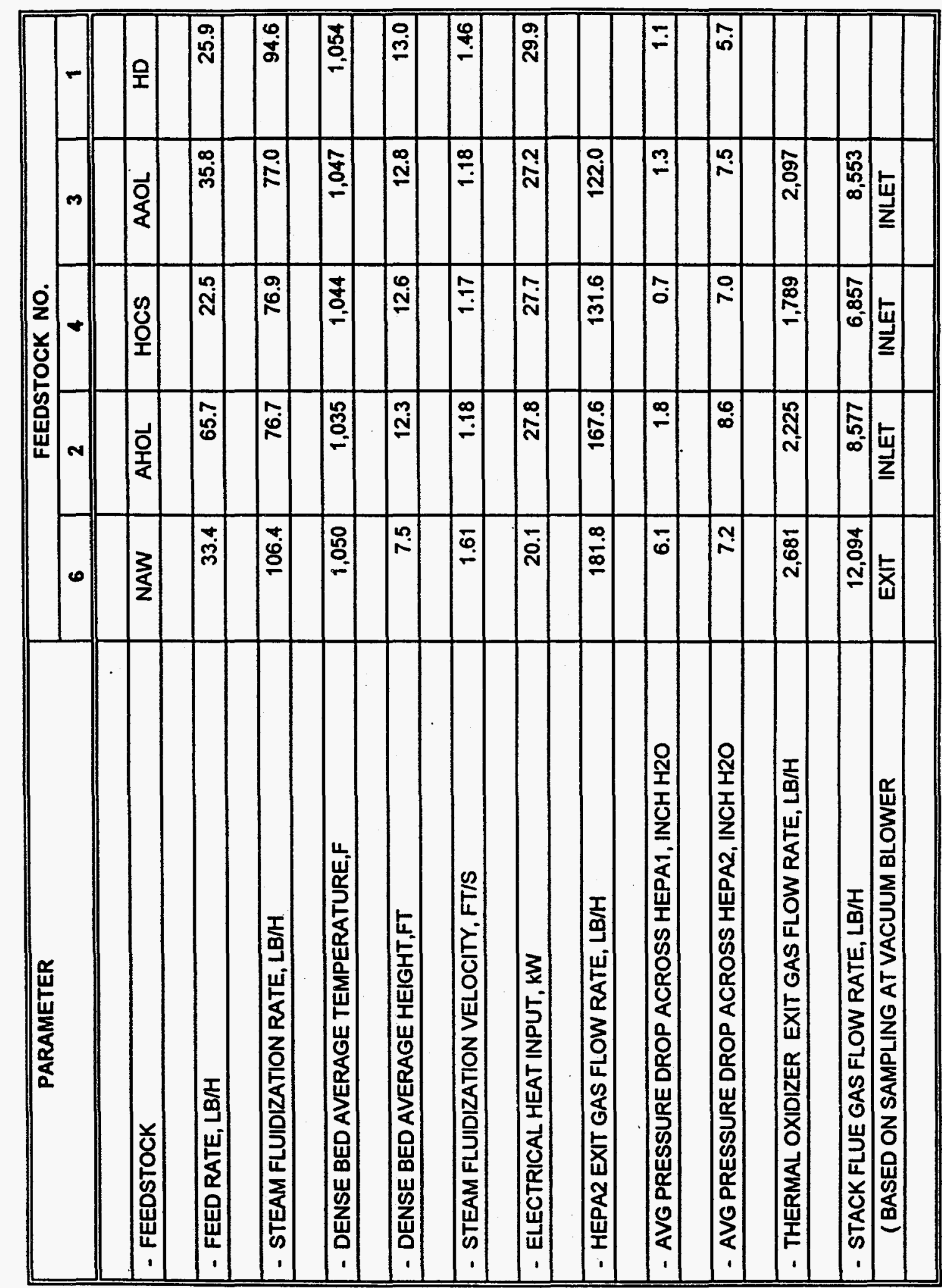




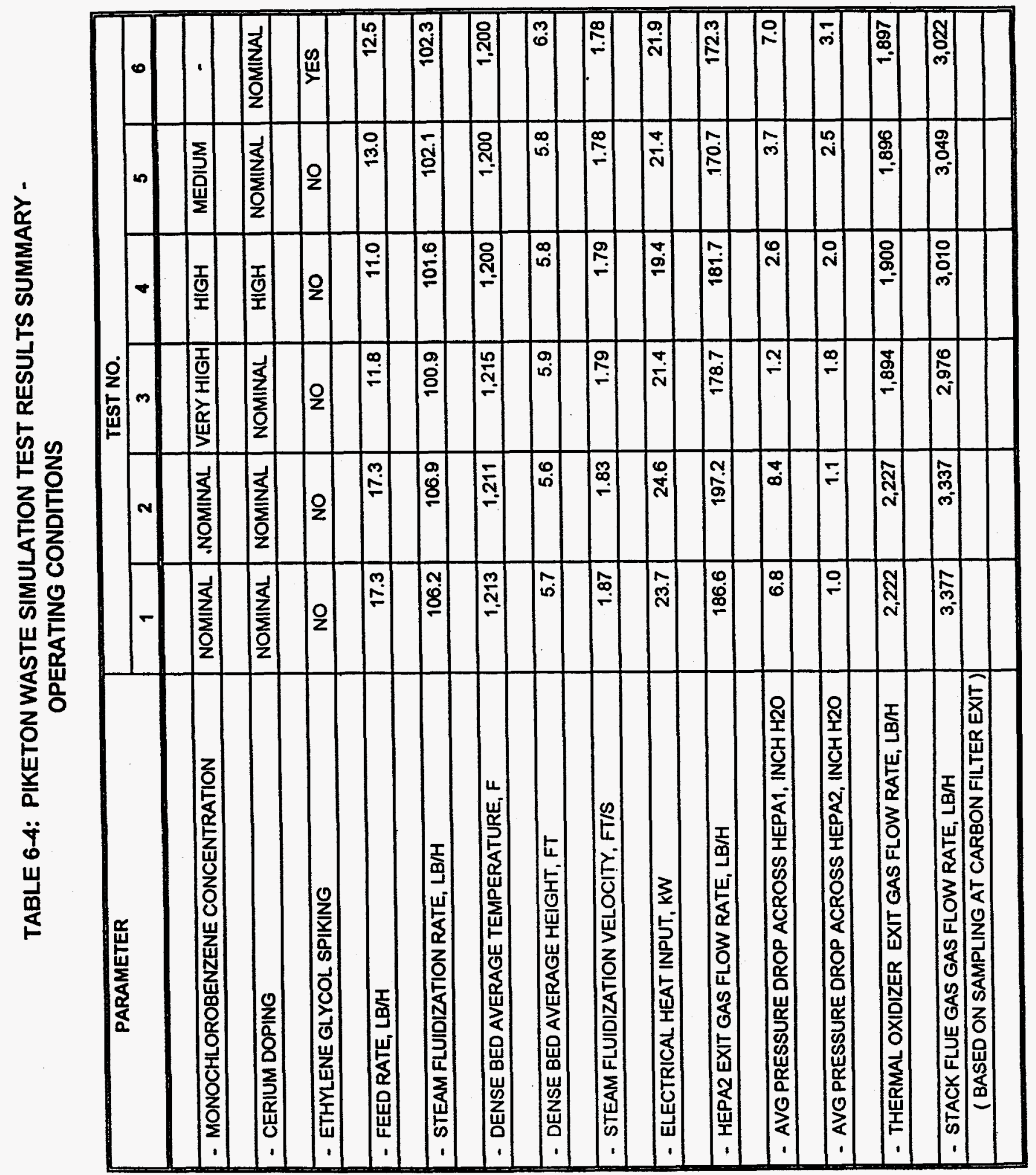




\section{TABLE 6-5: MULTIPLE ORGANIC FEEDSTOCK RETEST RESULTS SUMMARY OPERATING CONDITIONS}

\begin{tabular}{|c|c|c|c|}
\hline \multirow[t]{2}{*}{ PARAMETER } & \multicolumn{3}{|c|}{ TEST NO. } \\
\hline & R1 & $\mathbf{R 2}$ & $\mathbf{R 3}$ \\
\hline - MIXTURE FEED RATE, LB/H & $54.0-57.5$ & $20.1-20.6$ & $5.9-10.1$ \\
\hline - CARRIER ( SORBENT PAD ) FEED RATE, LB/H & - & 10.2 & 13.0 \\
\hline - TOTAL FEED RATE, LB/H & $54.0-57.5$ & $30.3-30.8$ & $18.9-23.1$ \\
\hline - STEAM FLUIDIZATION RATE, LB/H & 90.9 & 90.9 & 91.5 \\
\hline - DENSE BED AVERAGE TEMPERATURE,F & 1,050 & 1,050 & 1,053 \\
\hline - DENSE BED AVERAGE HEIGHT,FT & 13.1 & 13.7 & 13.4 \\
\hline - STEAM FLUIDIZATION VELOCITY, FTIS & 1.41 & 1.42 & 1.42 \\
\hline - ELECTRICAL HEAT INPUT, $\mathrm{kW}$ & 35.2 & 33.0 & 39.9 \\
\hline - AVG PRESSURE DROP ACROSS HEPA1, INCH H2O & 5.5 & 14.4 & 2.8 \\
\hline - AVG PRESSURE DROP ACROSS HEPA2, INCH H2O & 2.9 & 6.1 & 2.9 \\
\hline - THERMAL OXIDIZER EXIT GAS FLOW RATE, LB/H & 1,791 & 1,780 & 1,794 \\
\hline - STACK FLUE GAS GAS FLOW RATE, LB/H & 2,354 & 2,445 & 2,741 \\
\hline (BASED ON SAMPLING AT CARBON FILTER EXIT) & & & \\
\hline & & & \\
\hline
\end{tabular}


make composite samples of the following: feedstock, sand, bed drain catch, HT HEPA 1 catch, HT HEPA 2 catch, caustic, spray dryer catch, baghouse catch and LT HEPA catch.

Gas samples were taken downstream of the HT HEPA 2/upstream of the oxidizer and at the stack (either downstream or upstream of the I.D. roots blower). Gas sampling and analysis were performed by TRC Environmental Corporation.

Emissions testing included the following:

- VOCs - The presence of volatile organic compounds were determined by EPA Method 0030 (VOST). Samples were collected on Tenax absorbent cartridges and analyzed by GC/MS for toluene, tetrachloroethane, and vinyl chloride.

- Semi-Vols - Naphthalene, phenol, and 1-2-dichlorobenzene were sampled isokinetically in accordance with EPA Method 0010 using an XAD resin cartridge. The resin and impinger catch were solvent extracted and analyzed by GC/MS for the compounds of interest.

- $\quad$ Metals - $\mathrm{Pb}, \mathrm{Ni}, \mathrm{Cr}, \mathrm{Cd}, \mathrm{Ce}$, and $\mathrm{Cs}$ were sampled for isokinetically in accordance with EPA Method 29 using a $5 \% \mathrm{HNO}_{3} / 10 \% \mathrm{H}_{2} \mathrm{O}_{2}$ absorbing solution. The sample train was digested and analyzed by ICP for the elements of interest.

- $\quad \mathrm{HCl}$ - was sampled isokinetically in accordance with EPA Method 26A, analyzed by Ion Chromatograph (IC) for the compounds of interest.

Simultaneously with sample collection, TRC determined the volumetric flow rate of the system for use in mass emission rate calculations for compounds detected.

Solid Characterization. The analyses include ultimate analysis, RCRA metals, RCRA organics, radionuclides, and TCLP.

Gas Characterization. The steam reformer off-gas composition $\left(\mathrm{H}_{2}, \mathrm{CO}, \mathrm{C}_{\mathrm{x}} \mathrm{H}_{y}, \mathrm{H}_{2} \mathrm{O}\right.$, $\mathrm{CO}_{2}, \mathrm{HCl}, \mathrm{H}_{2} \mathrm{~S}$, VOC, SVOC, RCRA metals and radionuclides) and stack gas composition $\left(\mathrm{O}_{2}\right.$, $\mathrm{H}_{2} \mathrm{O}, \mathrm{CO}, \mathrm{SO}_{2}, \mathrm{HCl}, \mathrm{NO}_{x}, \mathrm{C}_{x} \mathrm{H}_{y}, \mathrm{VOC}$, SVOC, RCRA metals and radionuclides) were determined. The measurements included analysis for dioxins and furans in some cases. 


\subsubsection{Test Results}

Gas characterization data, solid characterization data and operational data were used along with Microsoft Excel spreadsheets to perform the data reduction.

Multiple Surrogate Tests. The main performance parameters are listed in Tables 6-6, 6-7 and 6-8. These results are very encouraging indeed. Table 6-9 furnishes data on the HT HEPA 2 exit gas composition and Table 6-10 indicates the stack flue gas composition.

Emissions of PCDD/PCDF were measured simultaneously at the HT HEPA2 exit and the stack during tests with Feedstocks 6 and 2. These data are summarized in Tables 6-11 through 6 14. The values given were all corrected to the $2,3,7,8-\mathrm{TCDD}$ toxicity equivalent basis.

The operating parameter trends for one of the test periods with the five feedstocks are shown in Figures 6-2 through 6-6 (the remaining charts are included as Appendix D). The following five parameters are included for each test:

- Steam reformer fluidization flow;

- $\quad$ Steam reformer freeboard pressure;

- $\quad$ HT HEPA1 and HT HEPA2 pressure drops;

- Temperature profiles (bed average, cyclone exit, HT HEPA2 exit and thermal oxidizer average temperatures); and

- Electrical heat input to the steam reformer.

Piketon Waste Tests. As mentioned previously, this was a continuous run with discrete samples taken at different time intervals. Most of the test campaign was conducted with the nominal composition feedstock (see Table 3-2). This composition corresponded very closely to that typically found in storage at the Piketon Gaseous Diffusion Plant site. Multiple samples were taken during two nine-hour runs with this feedstock and these are termed Tests 1 and 2. The monochlorobenzene (PCB surrogate) level was quite low in the nominal composition feedstock that the breakthrough concentration in the stack was found to be well below the instrument 


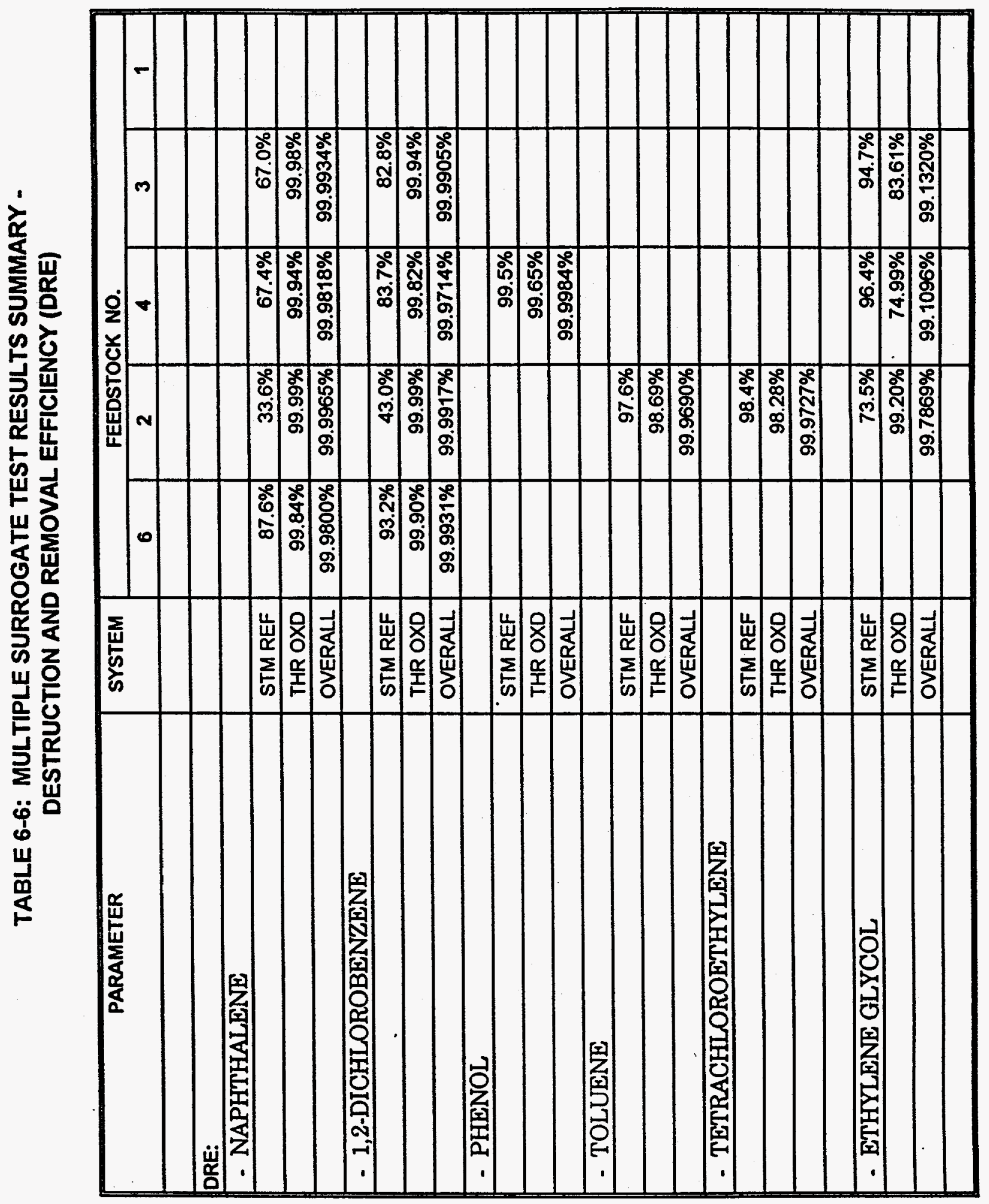




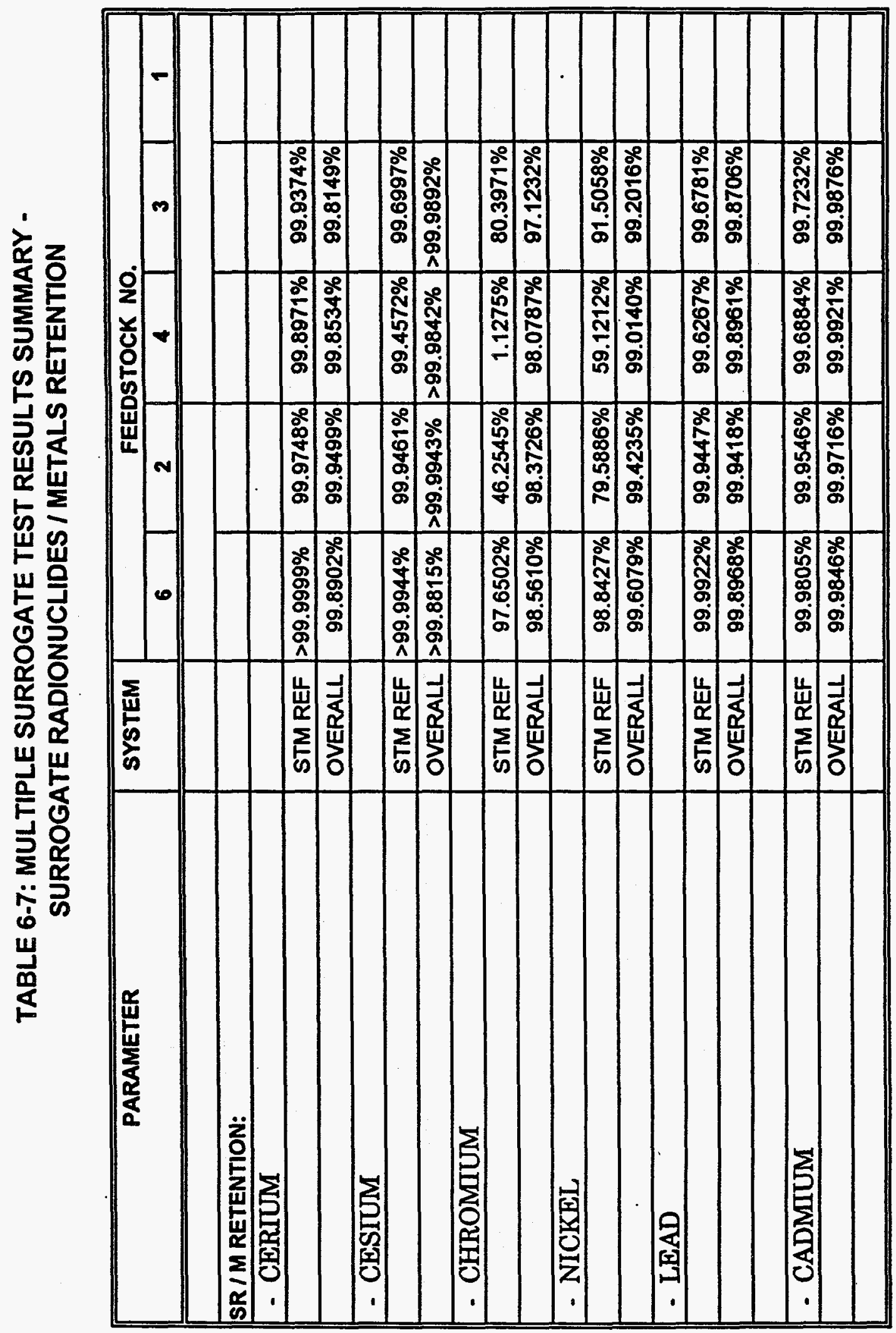




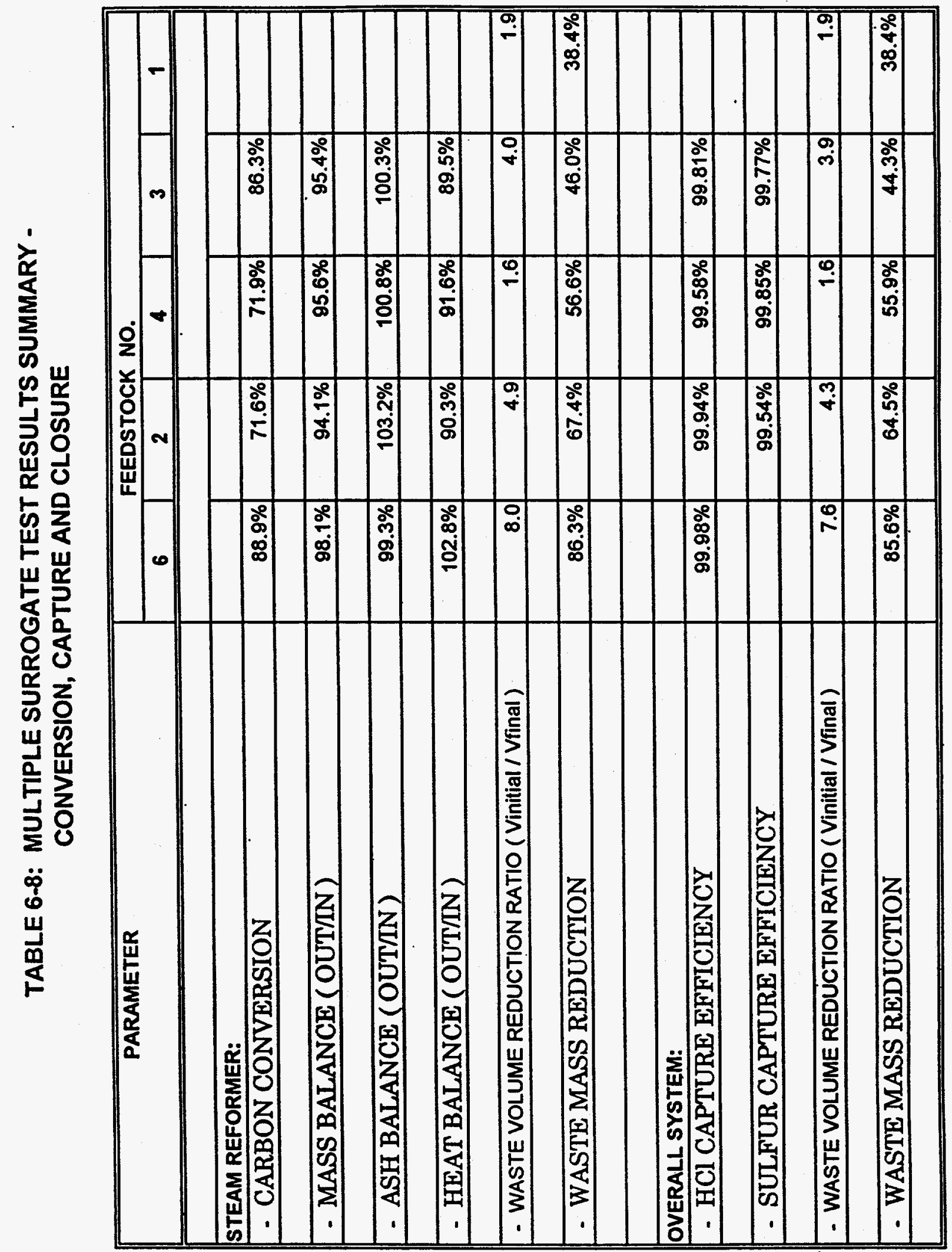




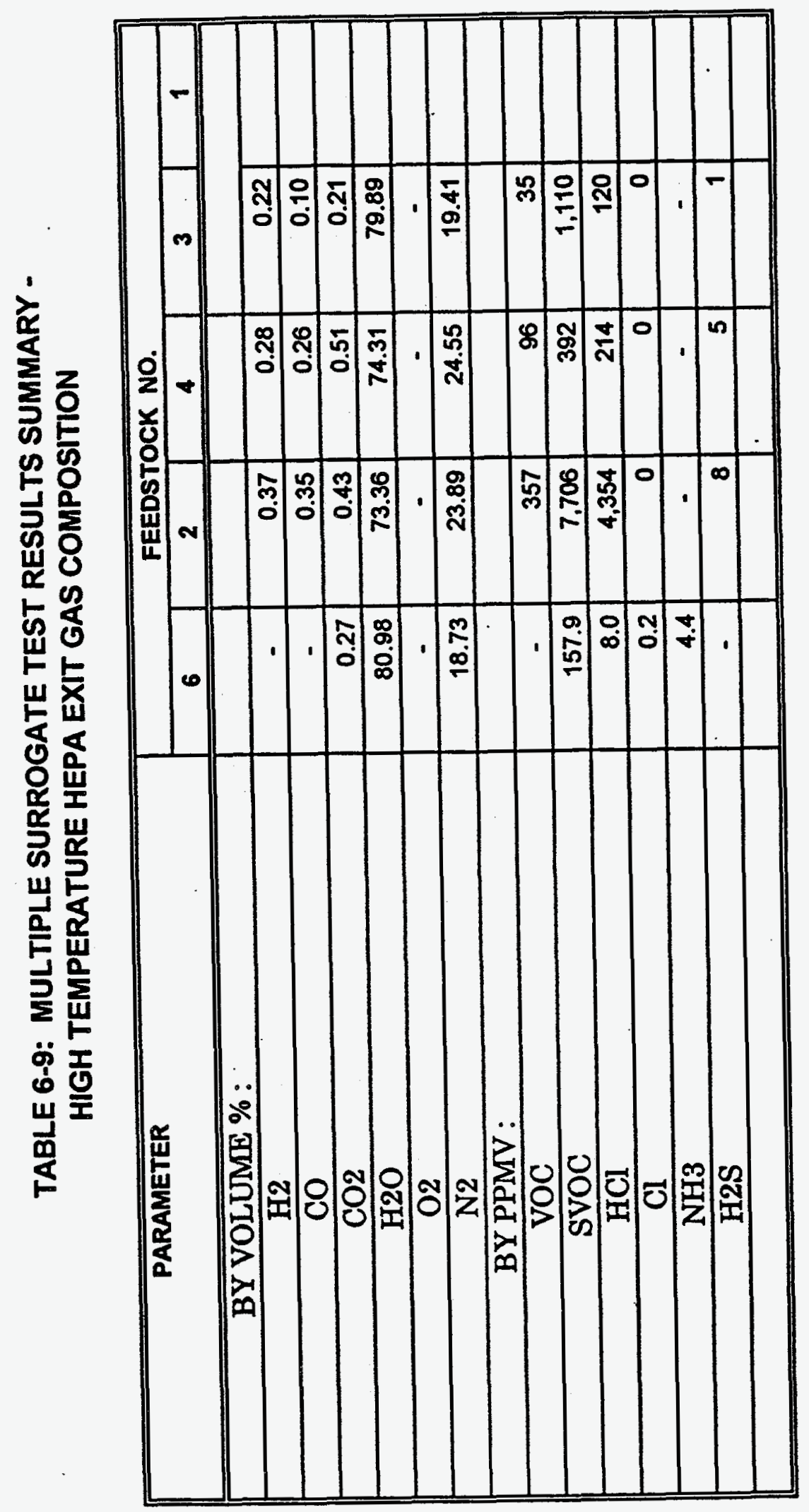




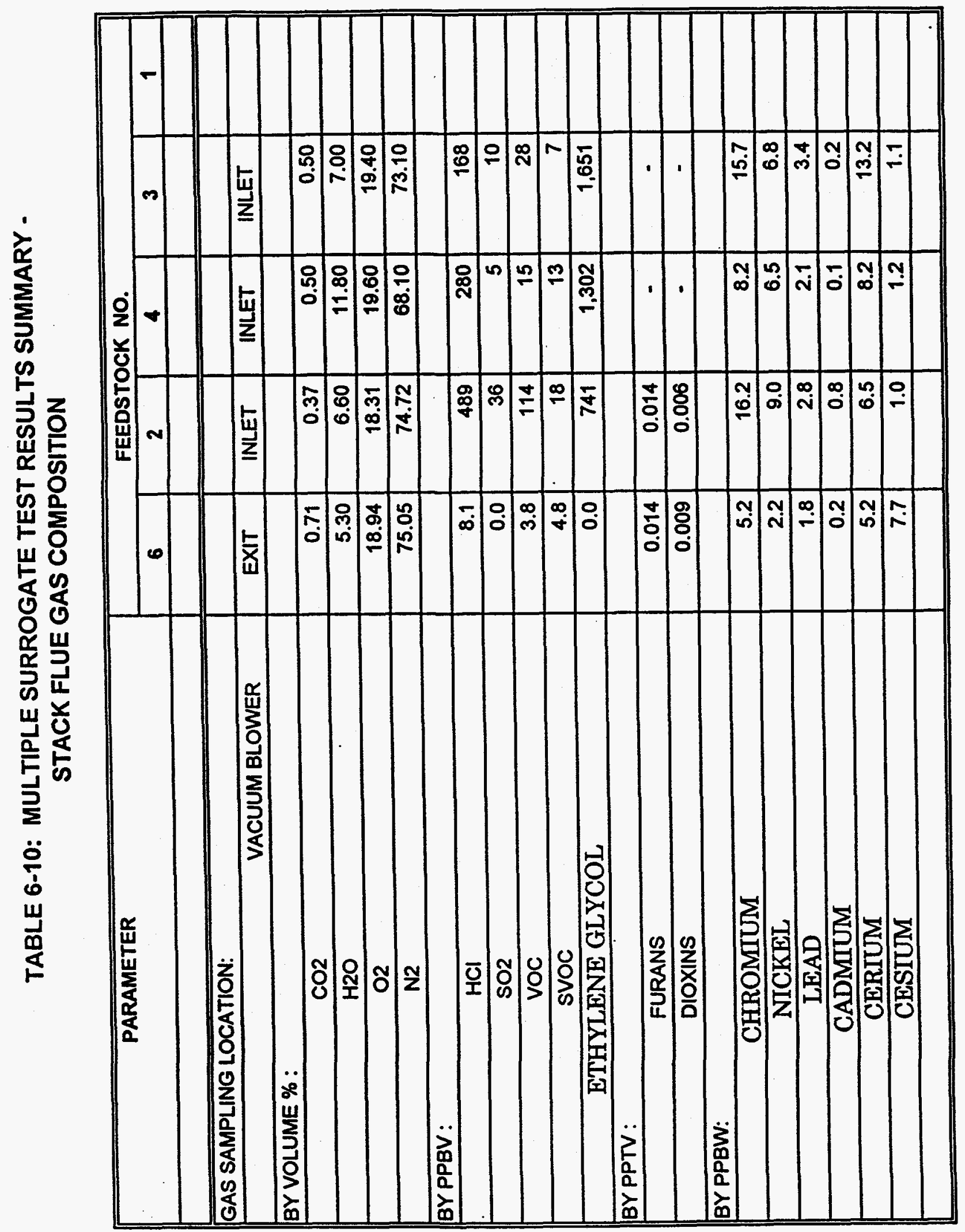




\section{TABLE 6-11: SUMMARY OF PCDD EMISSION RATES FEEDSTOCK NO. 6}

\begin{tabular}{|c|c|c|c|}
\hline \multirow{2}{*}{$\begin{array}{l}\text { TEST DESCRIPTION } \\
\text { DATE } \\
\text { TIME } \\
\text { TEST LOCATION } \\
\end{array}$} & & \multicolumn{2}{|c|}{ FEEDSTOCK NO. 6} \\
\hline & & $\begin{array}{r}2-13-97 \\
1504-2104 \\
\text { STACK } \\
\end{array}$ & $\begin{array}{c}\text { 2-13-97 } \\
\text { 1504-1704 } \\
\text { HEPA Exit }\end{array}$ \\
\hline Flow rate & $\begin{array}{l}\text { dscfml } \\
\text { dscf/hr } \\
\text { dscm/hr }\end{array}$ & $\begin{array}{r}2581 \\
154889 \\
4387\end{array}$ & $\begin{array}{r}11.1 \\
666 \\
19\end{array}$ \\
\hline Volume Sampled & dscm & 5.30 & 0.36 \\
\hline Oryeen Concentration & percent & 20.10 & 3.63 \\
\hline Compounds & & & \\
\hline $2,3,7,8 \mathrm{TCDD}$ & $\begin{array}{l}\mathrm{pg} \\
\mathrm{ug} / \mathrm{dscm} \\
\mathrm{ug} / \mathrm{dscm} @ 7 \% \mathrm{O} 2 \\
\mathrm{lbs} / \mathrm{hr}\end{array}$ & $\begin{array}{r}23.00 \\
4.34 \mathrm{E}-06 \\
7.55 \mathrm{E}-05 \\
4.20 \mathrm{E}-11\end{array}$ & $\begin{array}{r}3400.00 \\
9.34 \mathrm{E}-03 \\
7.52 \mathrm{E}-03 \\
3.88 \mathrm{E}-10\end{array}$ \\
\hline $1,2,3,7,8 \mathrm{PeCDD}$ & $\begin{array}{l}\text { pg } \\
\text { ug/dscm } \\
\text { ug/dscm@7\%02 } \\
\text { lbs/hr }\end{array}$ & $\begin{array}{r}3.45 \\
6.51 \mathrm{E}-07 \\
1.13 \mathrm{E}-05 \\
6.30 \mathrm{E}-12\end{array}$ & $\begin{array}{r}700.00 \\
1.92 \mathrm{E}-03 \\
1.55 \mathrm{E}-03 \\
8.00 \mathrm{E}-11\end{array}$ \\
\hline $1,2,3,4,7,8 \mathrm{HxCDD}$ & $\begin{array}{l}\mathrm{pg} \\
\text { ug/dscm } \\
\text { ug/dscm@7\%02 } \\
\text { lbs/hr }\end{array}$ & $\begin{array}{r}1.50 \\
2.83 \mathrm{E}-07 \\
4.92 \mathrm{E}-06 \\
2.74 \mathrm{E}-12\end{array}$ & $\begin{array}{r}21.00 \\
5.77 \mathrm{E}-05 \\
4.64 \mathrm{E}-05 \\
2.40 \mathrm{E}-12\end{array}$ \\
\hline $\mid 1,2,3,6,7,8 \mathrm{HxCDD}$ & $\begin{array}{l}\text { pg } \\
\text { ug/dscm } \\
\text { ug/dscm@7\%O2 } \\
\text { lbs/hr }\end{array}$ & $\begin{array}{r}0.31 \\
5.85 \mathrm{E}-08 \\
1.02 \mathrm{E}-06 \\
5.66 \mathrm{E}-13\end{array}$ & $\begin{array}{r}20.00 \\
5.49 \mathrm{E}-05 \\
4.42 \mathrm{E}-05 \\
2.28 \mathrm{E}-12\end{array}$ \\
\hline $1,2,3,7,8,9 \mathrm{HxCDD}$ & $\begin{array}{l}\mathrm{pg} \\
\text { ug/dscm } \\
\text { ug/dscm@7\%O2 } \\
\mathrm{lbs} / \mathrm{hr}\end{array}$ & $\begin{array}{r}0.41 \\
7.74 \mathrm{E}-08 \\
1.35 \mathrm{E}-06 \\
7.49 \mathrm{E}-13\end{array}$ & $\begin{array}{r}30.00 \\
8.24 \mathrm{E}-05 \\
6.63 \mathrm{E}-05 \\
3.43 \mathrm{E}-12\end{array}$ \\
\hline $1,2,3,4,6,7,8 \mathrm{HpCDD}$ & $\begin{array}{l}\text { pg } \\
\text { ug/dscm } \\
\text { ug/dscm@7\%O2 } \\
\text { lbs/hr }\end{array}$ & $\begin{array}{r}0.11 \\
2.08 \mathrm{E}-08 \\
3.61 \mathrm{E}-07 \\
2.01 \mathrm{E}-13\end{array}$ & $\begin{array}{r}5.00 \\
1.37 \mathrm{E}-05 \\
1.11 \mathrm{E}-05 \\
5.71 \mathrm{E}-13 \\
\end{array}$ \\
\hline
\end{tabular}




\section{TABLE 6-11: SUMMARY OF PCDD EMISSION RATES \\ FEEDSTOCK NO. 6 \\ (Continued)}

\begin{tabular}{|c|c|c|c|}
\hline \multirow{2}{*}{$\begin{array}{l}\text { TEST DESCRIPTION } \\
\text { DATE } \\
\text { TIME } \\
\text { TEST LOCATION }\end{array}$} & & \multicolumn{2}{|c|}{ FEEDSTOCK NO. 6} \\
\hline & & $\begin{array}{r}2-13-97 \\
1504-2104 \\
\text { STACK } \\
\end{array}$ & 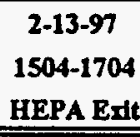 \\
\hline Total OCDD & $\begin{array}{l}\text { pg } \\
\text { ug/dscm } \\
\text { ug/dscm@7\%O2 } \\
\text { lbs/hr }\end{array}$ & $\begin{array}{r}0.05 \\
9.06 \mathrm{E}-09 \\
1.57 \mathrm{E}-07 \\
8.77 \mathrm{E}-14\end{array}$ & $\begin{array}{r}1.70 \\
4.67 \mathrm{E}-06 \\
3.76 \mathrm{E}-06 \\
1.94 \mathrm{E}-13\end{array}$ \\
\hline Total PCDD & $\begin{array}{l}\mathrm{pg} \\
\text { ug/dscm } \\
\text { ug/dscm@7\%O2 } \\
\text { lbs/hr }\end{array}$ & $\begin{array}{r}28.83 \\
5.44 \mathrm{E}-06 \\
9.46 \mathrm{E}-05 \\
5.26 \mathrm{E}-11\end{array}$ & $\begin{array}{r}4177.70 \\
1.15 \mathrm{E}-02 \\
9.24 \mathrm{E}-03 \\
4.77 \mathrm{E}-10\end{array}$ \\
\hline
\end{tabular}

1- Actual cubic feet per minute (acfm) was measured at the stack exhaust by method 1 and 2, but was not measured at HEPA Exit.

2 - dscfm $=\mathrm{dry}$ standard cubic fect per minute at $68^{\circ} \mathrm{F}$ and $29.92 \mathrm{in.} \mathrm{Hg}$

3- Dry gas flow rate at HEPA Exit was calculated from the gas moisture fraction measured with Method 0050 and the total steam input. 
TABLE 6-12: SUMMARY OF PCDF EMISSION RATES

FEEDSTOCK NO. 6

\begin{tabular}{|c|c|c|c|}
\hline \multirow{2}{*}{$\begin{array}{l}\text { TEST DESCRIPTION } \\
\text { DATE } \\
\text { TIME } \\
\text { LOCATION }\end{array}$} & & \multicolumn{2}{|c|}{ FEEDSTOCK'NO.6 } \\
\hline & & $\begin{array}{c}2-13-97 \\
1504-2104 \\
\text { STACK } \\
\end{array}$ & 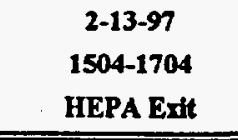 \\
\hline Flow rate & $\begin{array}{l}\text { dscfm' } \\
\text { dscf/hr } \\
\text { dscm/hr }\end{array}$ & $\begin{array}{r}2581 \\
154889 \\
4387\end{array}$ & $\begin{array}{r}11.1 \\
666 \\
19\end{array}$ \\
\hline Volume Sampled & dscm & 5.30 & 0.36 \\
\hline Oxven Concentration & percent & 20.10 & 3.63 \\
\hline Compound & & & \\
\hline $2,3,7,8$ TCDF & $\begin{array}{l}\text { pg } \\
\text { ug/dscm } \\
\text { ug/dscm@7\%O2 } \\
\mathrm{lbs} / \mathrm{hr}\end{array}$ & $\begin{array}{r}9.40 \\
1.77 \mathrm{E}-06 \\
3.08 \mathrm{E}-05 \\
1.72 \mathrm{E}-11\end{array}$ & $\begin{array}{r}2500.00 \\
6.87 E-03 \\
5.53 E-03 \\
2.86 E-10\end{array}$ \\
\hline $1,2,3,7,8 \mathrm{PeCDF}$ & $\begin{array}{l}\mathrm{pg} \\
\text { ug/dscm } \\
\text { ug/dscm@7\%02 } \\
\mathrm{lbs} / \mathrm{hr}\end{array}$ & $\begin{array}{r}0.39 \\
7.36 \mathrm{E}-08 \\
1.28 \mathrm{E}-06 \\
7.12 \mathrm{E}-13\end{array}$ & $\begin{array}{r}65.00 \\
1.79 \mathrm{E}-04 \\
1.44 \mathrm{E}-04 \\
7.42 \mathrm{E}-12\end{array}$ \\
\hline $2,3,4,7,8 \mathrm{PeCDF}$ & $\begin{array}{l}\text { pg } \\
\text { ug/dscm } \\
\text { ug/dscm@7\%02 } \\
\text { lbs/hr }\end{array}$ & $\begin{array}{r}2.90 \\
5.48 \mathrm{E}-07 \\
9.51 \mathrm{E}-06 \\
5.30 \mathrm{E}-12\end{array}$ & $\begin{array}{r}345.00 \\
9.48 \mathrm{E}-04 \\
7.63 \mathrm{E}-04 \\
3.94 \mathrm{E}-11\end{array}$ \\
\hline $1,2,3,4,7,8 \mathrm{HxCDF}$ & $\begin{array}{l}\mathrm{pg} \\
\mathrm{ug} / \mathrm{dscm} \\
\mathrm{ug} / \mathrm{dscm} @ 7 \% 02 \\
\mathrm{lbs} / \mathrm{hr}\end{array}$ & $\begin{array}{r}0.32 \\
6.04 \mathrm{E}-08 \\
1.05 \mathrm{E}-06 \\
5.84 \mathrm{E}-13\end{array}$ & $\begin{array}{r}17.00 \\
4.67 \mathrm{E}-05 \\
3.76 \mathrm{E}-05 \\
1.94 \mathrm{E}-12\end{array}$ \\
\hline $1,2,3,6,7,8 \mathrm{HxCDF}$ & $\begin{array}{l}\mathrm{pg} \\
\mathrm{ug} / \mathrm{dscm} \\
\mathrm{ug} / \mathrm{dscm} @ 7 \% \mathrm{O} 2 \\
\mathrm{lbs} / \mathrm{hr}\end{array}$ & $\begin{array}{r}0.21 \\
3.97 \mathrm{E}-08 \\
6.89 \mathrm{E}-07 \\
3.83 \mathrm{E}-13\end{array}$ & $\begin{array}{r}12.00 \\
3.30 \mathrm{E}-05 \\
2.65 \mathrm{E}-05 \\
1.37 \mathrm{E}-12\end{array}$ \\
\hline $1,2,3,7,8,9 \mathrm{HxCDF}$ & $\begin{array}{l}\text { pg } \\
\text { ug/dscm } \\
\text { ug/dscm@7\%02 } \\
\text { lbs/hr }\end{array}$ & $\begin{array}{r}1.70 \\
3.21 \mathrm{E}-07 \\
5.58 \mathrm{E}-06 \\
3.10 \mathrm{E}-12 \\
\end{array}$ & $\begin{array}{r}1.60 \\
4.39 \mathrm{E}-06 \\
3.54 \mathrm{E}-06 \\
1.83 \mathrm{E}-13 \\
\end{array}$ \\
\hline
\end{tabular}




\section{TABLE 6-12: SUMMARY OF PCDF EMISSION RATES \\ FEEDSTOCK NO. 6 \\ (Continued)}

\begin{tabular}{|c|c|c|c|}
\hline \multirow{2}{*}{$\begin{array}{l}\text { TEST DESCRIPTION } \\
\text { DATE } \\
\text { TIME } \\
\text { LOCATION }\end{array}$} & & \multicolumn{2}{|c|}{ FEEDSTOCK NO.6 } \\
\hline & & $\begin{array}{c}\text { 2-13-97 } \\
1504-2104 \\
\text { STACK }\end{array}$ & $\begin{array}{c}\text { 2-13-97 } \\
\text { 1504-1704 } \\
\text { HEPA Extt }\end{array}$ \\
\hline $2,3,4,6,7,8 \mathrm{HxCDF}$ & $\begin{array}{l}\mathrm{pg} \\
\text { ug/dscm } \\
\text { ug/dscm@7\%O2 } \\
\mathrm{lbs} / \mathrm{hr}\end{array}$ & $\begin{array}{rr}< & 0.32 \\
< & 6.04 \mathrm{E}-08 \\
< & 1.05 \mathrm{E}-06 \\
< & 5.84 \mathrm{E}-13\end{array}$ & $\begin{array}{r}6.50 \\
1.79 \mathrm{E}-05 \\
1.44 \mathrm{E}-05 \\
7.42 \mathrm{E}-13\end{array}$ \\
\hline $1,2,3,4,6,7,8 \mathrm{HpCDF}$ & $\begin{array}{l}\text { pg } \\
\text { ug/dscm } \\
\text { ug/dscm@7\%O2 } \\
\text { lbs } / \mathrm{hr}\end{array}$ & $\begin{array}{r}0.06 \\
1.04 \mathrm{E}-08 \\
1.80 \mathrm{E}-07 \\
1.00 \mathrm{E}-13\end{array}$ & $\begin{array}{r}1.10 \\
3.02 \mathrm{E}-06 \\
2.43 \mathrm{E}-06 \\
1.26 \mathrm{E}-13\end{array}$ \\
\hline $1,2,3,4,7,8,9 \mathrm{HpCDF}$ & $\begin{array}{l}\mathrm{pg} \\
\text { ug/dscm } \\
\text { ug/dscm@7\%O2 } \\
\mathrm{lbs} / \mathrm{hr}\end{array}$ & $\begin{array}{r}0.04 \\
8.31 E-09 \\
1.44 E-07 \\
8.03 E-14\end{array}$ & $\begin{array}{r}0.57 \\
1.57 \mathrm{E}-06 \\
1.26 \mathrm{E}-06 \\
6.51 \mathrm{E}-14\end{array}$ \\
\hline Total OCDF & $\begin{array}{l}\text { pg } \\
\text { ug/dscm } \\
\text { ug/dscm@7\%02 } \\
\text { lbs/hr }\end{array}$ & $\begin{array}{r}0.01 \\
1.40 \mathrm{E}-09 \\
2.43 \mathrm{E}-08 \\
1.35 \mathrm{E}-14\end{array}$ & $\begin{array}{r}0.04 \\
1.15 \mathrm{E}-07 \\
9.29 \mathrm{E}-08 \\
4.80 \mathrm{E}-15\end{array}$ \\
\hline Total PCDF & $\begin{array}{l}\mathrm{pg} \\
\text { ug/dscm } \\
\text { ug/dscm@7\%O2 } \\
\mathrm{lbs} / \mathrm{hr}\end{array}$ & $\begin{array}{r}15.35 \\
2.90 \mathrm{E}-06 \\
5.03 \mathrm{E}-05 \\
2.80 \mathrm{E}-11\end{array}$ & $\begin{array}{r}2948.81 \\
8.10 \mathrm{E}-03 \\
6.52 \mathrm{E}-03 \\
3.37 \mathrm{E}-10\end{array}$ \\
\hline
\end{tabular}

1 - Actual cubic feet per minute (acfin) was measured at the stack exhaust by method 1 and 2, but was not measured at HEPA Exit.

2 - dsefm $=$ dry standard cubic feet per minute at $68^{\circ} \mathrm{F}$ and $29.92 \mathrm{in.} \mathrm{Hg}$.

3 - Dry gas flow rate at HEPA Exit was calculated from the gas moisture fraction measured with Method 0050 and the total ateam input. 
TABLE 6-13: SUMMARY OF PCDD EMISSION RATES

FEEDSTOCK NO. 2

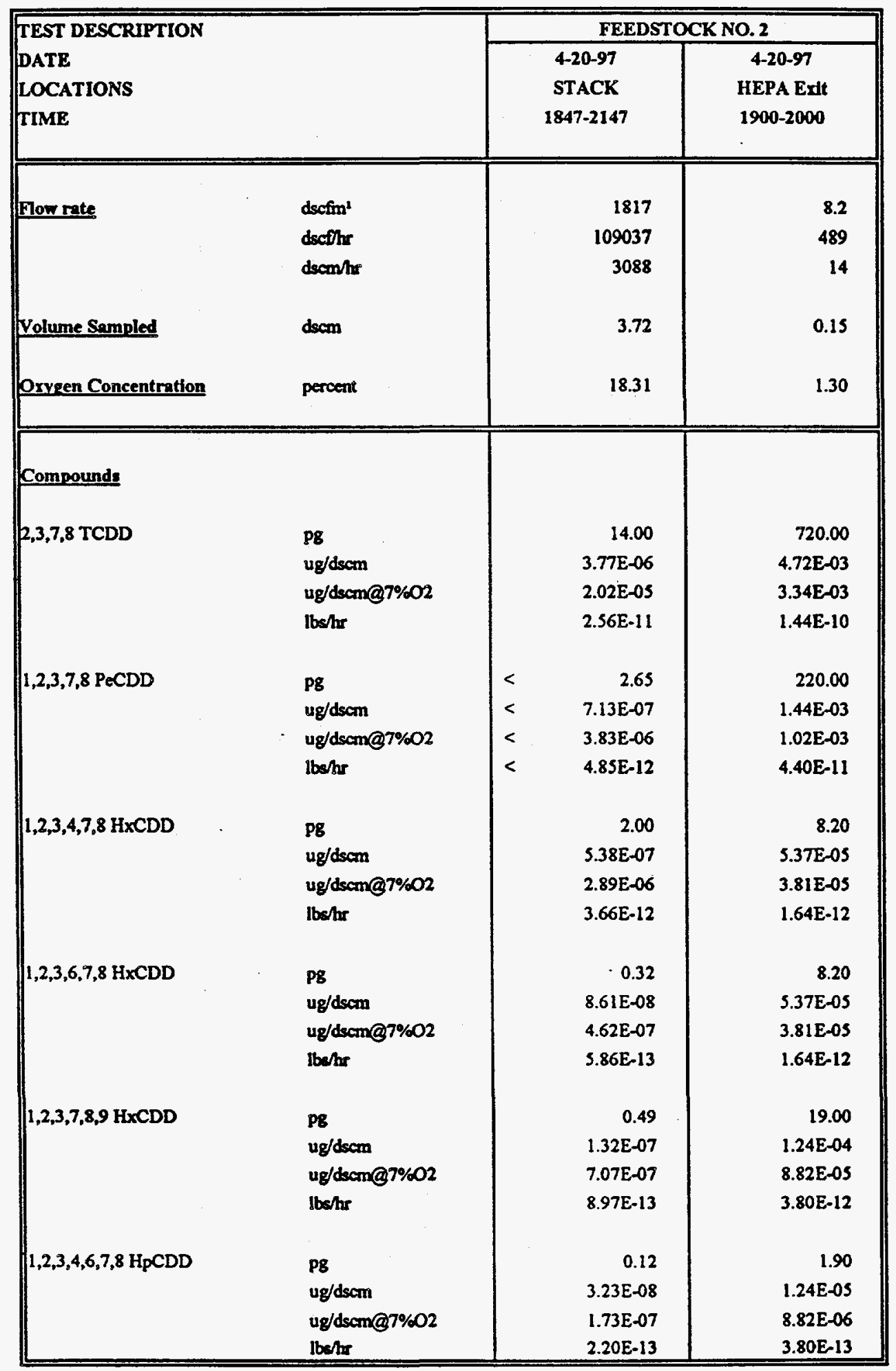




\section{TABLE 6-13: SUMMARY OF PCDD EMISSION RATES}

FEEDSTOCK NO. 2

(Continued)

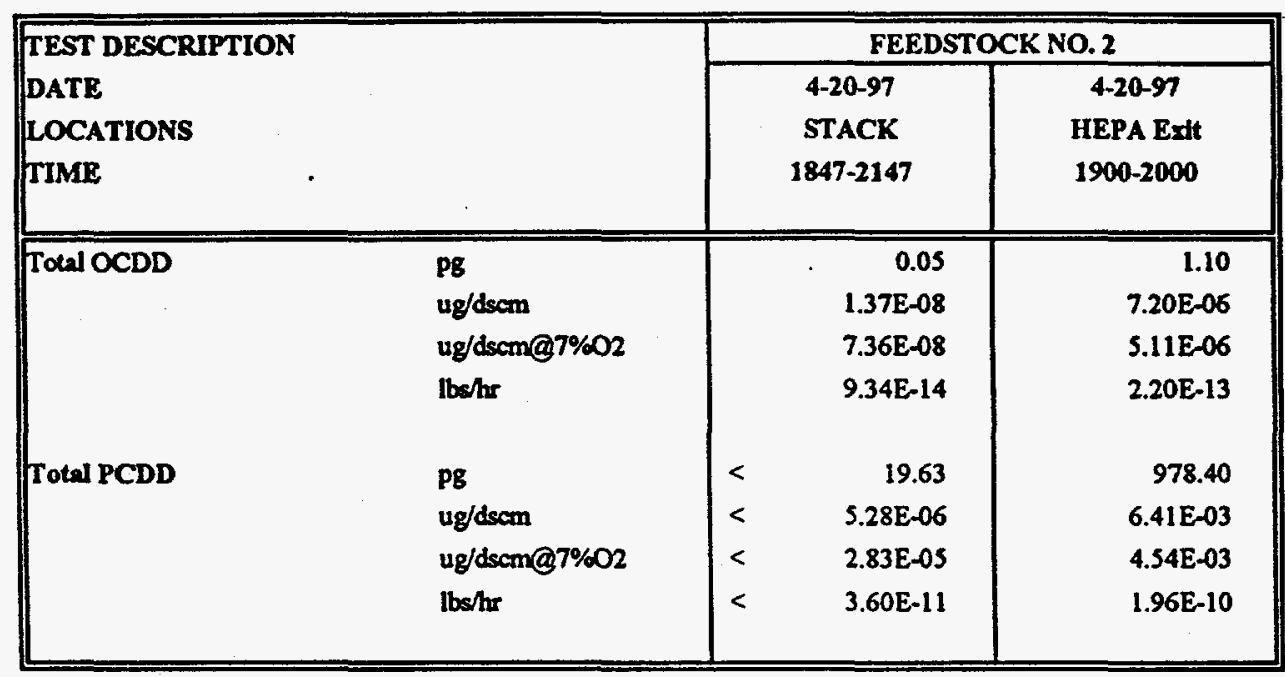

1 - Actual cubic fet per minute (acfin) was measured at the stack exhaust by method 1 and 2, but was not measured at HEPA Exit.

2 - dscfin = dry standard cubic feet per minute at $68^{\circ} \mathrm{F}$ and $29.92 \mathrm{in} . \mathrm{Hg}$.

3-Dry gas flow rate at HEPA Exit was calculated from the gas moisture fraction measured with Method 0050 and the total steam input. 
TABLE 6-14: SUMMARY OF PCDF EMISSION RATES

FEEDSTOCK NO. 2 .

\begin{tabular}{|c|c|c|c|}
\hline \multirow{2}{*}{$\begin{array}{l}\text { TEST DESCRIPTION } \\
\text { DATE } \\
\text { Location } \\
\text { TIME }\end{array}$} & & \multicolumn{2}{|c|}{ FEEDSTOCK NO. 2} \\
\hline & & $\begin{array}{c}4-20-97 \\
\text { STACK } \\
1847-2147\end{array}$ & $\begin{array}{c}4-20-97 \\
\text { HEPA Exit } \\
1900-2000\end{array}$ \\
\hline Flow rate & $\begin{array}{l}\text { dsefm' } \\
\text { dscethr } \\
\text { dscm/hr }\end{array}$ & $\begin{array}{r}1817 \\
109037 \\
3088\end{array}$ & $\begin{array}{r}8 \\
489 \\
13.85\end{array}$ \\
\hline Volume Sampled & $\mathrm{dscm}$ & 3.72 & 0.15 \\
\hline Oxyen Concentration & percent & 18.31 & 1.30 \\
\hline Compound & & & \\
\hline $2,3,7,8 \mathrm{TCDF}$ & $\begin{array}{l}\mathrm{pg} \\
\text { ug/dscm } \\
\text { ug/dscm } 97 \% 02 \\
\text { lbshr }\end{array}$ & $\begin{array}{r}3.10 \\
8.34 \mathrm{E}-07 \\
4.48 \mathrm{E}-06 \\
5.68 \mathrm{E}-12\end{array}$ & $\begin{array}{r}3900.00 \\
2.55 \mathrm{E}-02 \\
1.81 \mathrm{E}-02 \\
7.80 \mathrm{E}-10\end{array}$ \\
\hline $1,2,3,7,8 \mathrm{PeCDF}$ & $\begin{array}{l}\mathrm{pg} \\
\text { ug/dscm } \\
\text { ug/dscma } 7 \% 02 \\
\mathrm{lbs} / \mathrm{hr}\end{array}$ & $\begin{array}{r}0.41 \\
1.10 \mathrm{E}-07 \\
5.92 \mathrm{E}-07 \\
7.51 \mathrm{E}-13\end{array}$ & $\begin{array}{r}205.00 \\
1.34 \mathrm{E}-03 \\
9.52 \mathrm{E}-04 \\
4.10 \mathrm{E}-11\end{array}$ \\
\hline $2,3,4,7,8 \mathrm{PeCDF}$ & $\begin{array}{l}\text { pg } \\
\text { ug/dscm } \\
\text { ug/dscm@7\%o2 } \\
\text { lbshr }\end{array}$ & $\begin{array}{r}3.65 \\
9.82 \mathrm{E}-07 \\
5.27 \mathrm{E}-06 \\
6.69 \mathrm{E}-12\end{array}$ & $\begin{array}{r}2350.00 \\
1.54 \mathrm{E}-02 \\
1.09 \mathrm{E}-02 \\
4.70 \mathrm{E}-10\end{array}$ \\
\hline $1,2,3,4,7,8 \mathrm{HXCDF}$ & $\begin{array}{l}\mathrm{pg} \\
\text { ug/dsem } \\
\text { ug/dscm@7\%202 } \\
\mathrm{lbs} / \mathrm{hr}\end{array}$ & $\begin{array}{r}0.91 \\
2.45 \mathrm{E}-07 \\
1.31 \mathrm{E}-06 \\
1.67 \mathrm{E}-12\end{array}$ & $\begin{array}{r}160.00 \\
1.05 \mathrm{E}-03 \\
7.43 \mathrm{E}-04 \\
3.20 \mathrm{E}-11\end{array}$ \\
\hline $1,2,3,6,7,8 \mathrm{HxCDF}$ & $\begin{array}{l}\mathrm{PB} \\
\mathrm{ug} / \mathrm{dscm} \\
\text { ug/dscm@g } 7 \% 02 \\
\text { lbe/hr }\end{array}$ & $\begin{array}{r}0.58 \\
1.56 \mathrm{E}-07 \\
8.37 \mathrm{E}-07 \\
1.06 \mathrm{E}-12\end{array}$ & $\begin{array}{r}82.00 \\
5.37 \mathrm{E}-04 \\
3.81 \mathrm{E}-04 \\
1.64 \mathrm{E}-11\end{array}$ \\
\hline $1,2,3,7,8,9 \mathrm{H} \times \mathrm{CDF}$ & $\begin{array}{l}\mathrm{PB} \\
\text { ug/decm } \\
\text { ug/dsem } 97 \% 02 \\
\text { lbs/hr }\end{array}$ & $\begin{array}{r}0.54 \\
1.45 E-07 \\
7.80 E-07 \\
9.89 E-13\end{array}$ & $\begin{array}{r}9.40 \\
6.16 \mathrm{E}-05 \\
4.37 \mathrm{E}-05 \\
1.88 \mathrm{E}-12 \\
\end{array}$ \\
\hline
\end{tabular}




\section{TABLE 6-14: SUMMARY OF PCDF EMISSION RATES}

FEEDSTOCK NO. 2

\section{( Continued)}

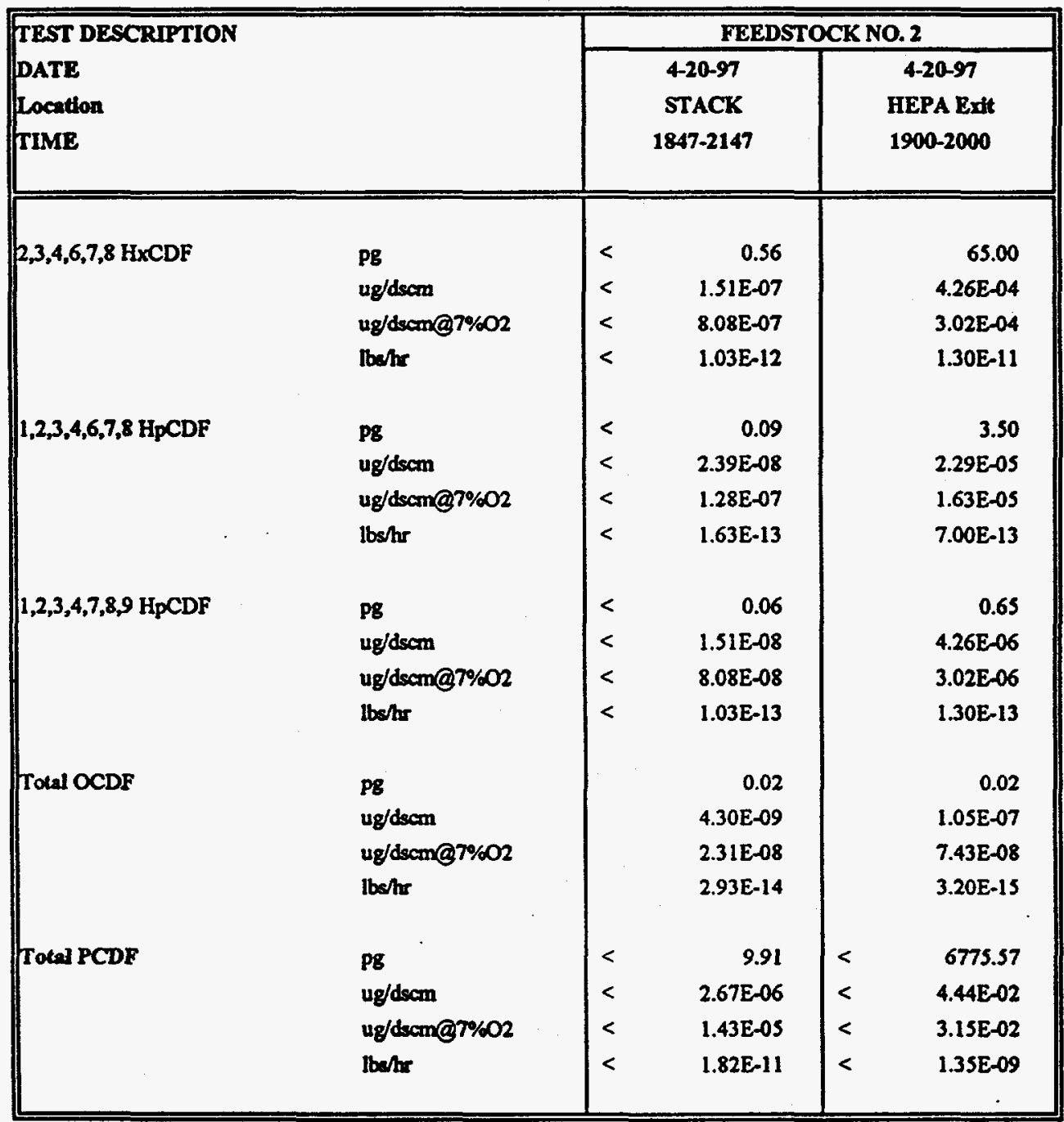

1 - Actual cubic fet per minute ( and 2, but was not measured at the HEPA Exit.

2 - dscin = dry standard cubic feet per Minute at $68^{\circ} \mathrm{F}$ and $29.92 \mathrm{in.} \mathrm{Hg}$.

3 - Dry gas flow rate at HEPA Exit was calculated from the gas moisture fraction measured with Method 0050 and the total ateam input See appendix A for calculation 


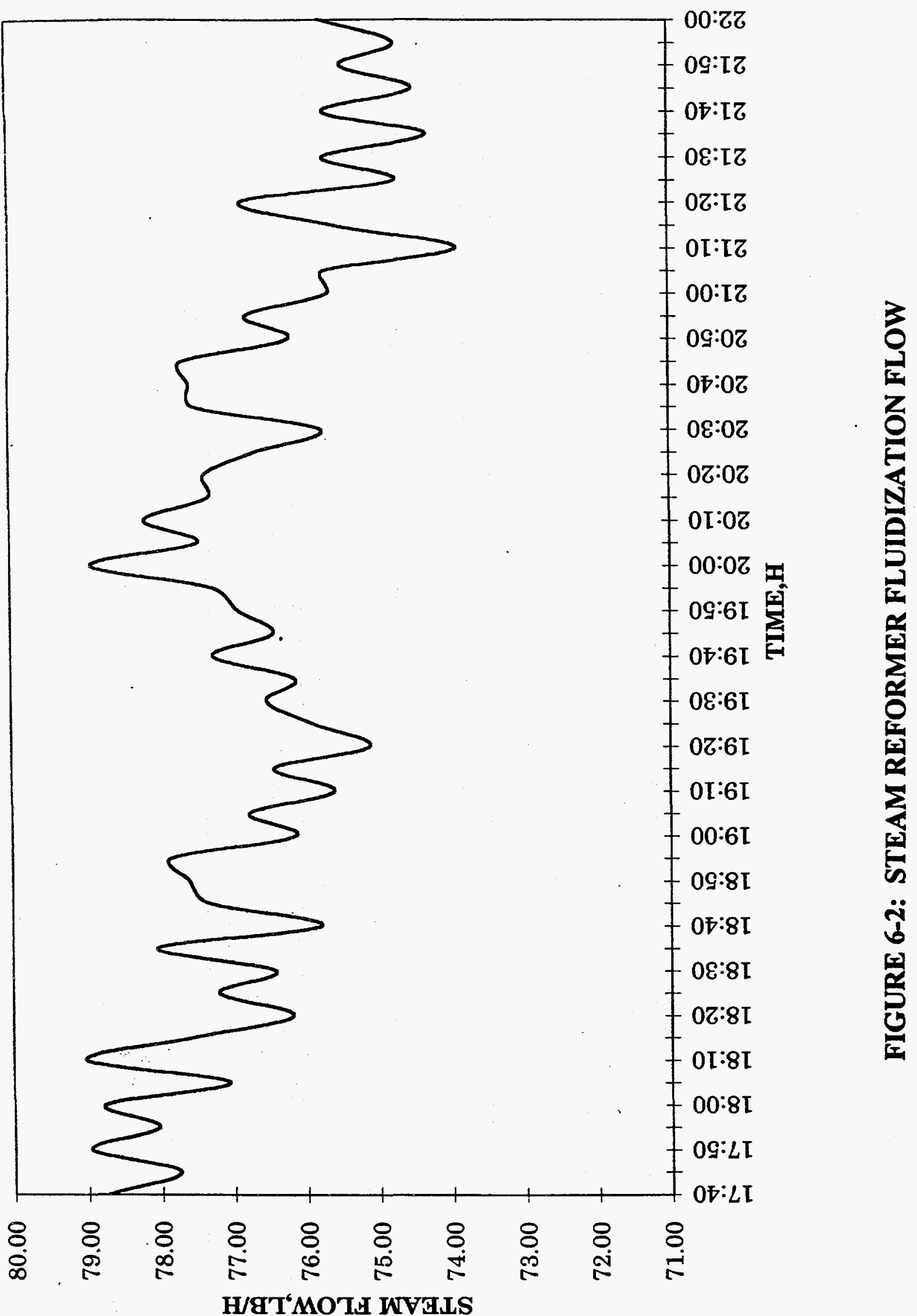




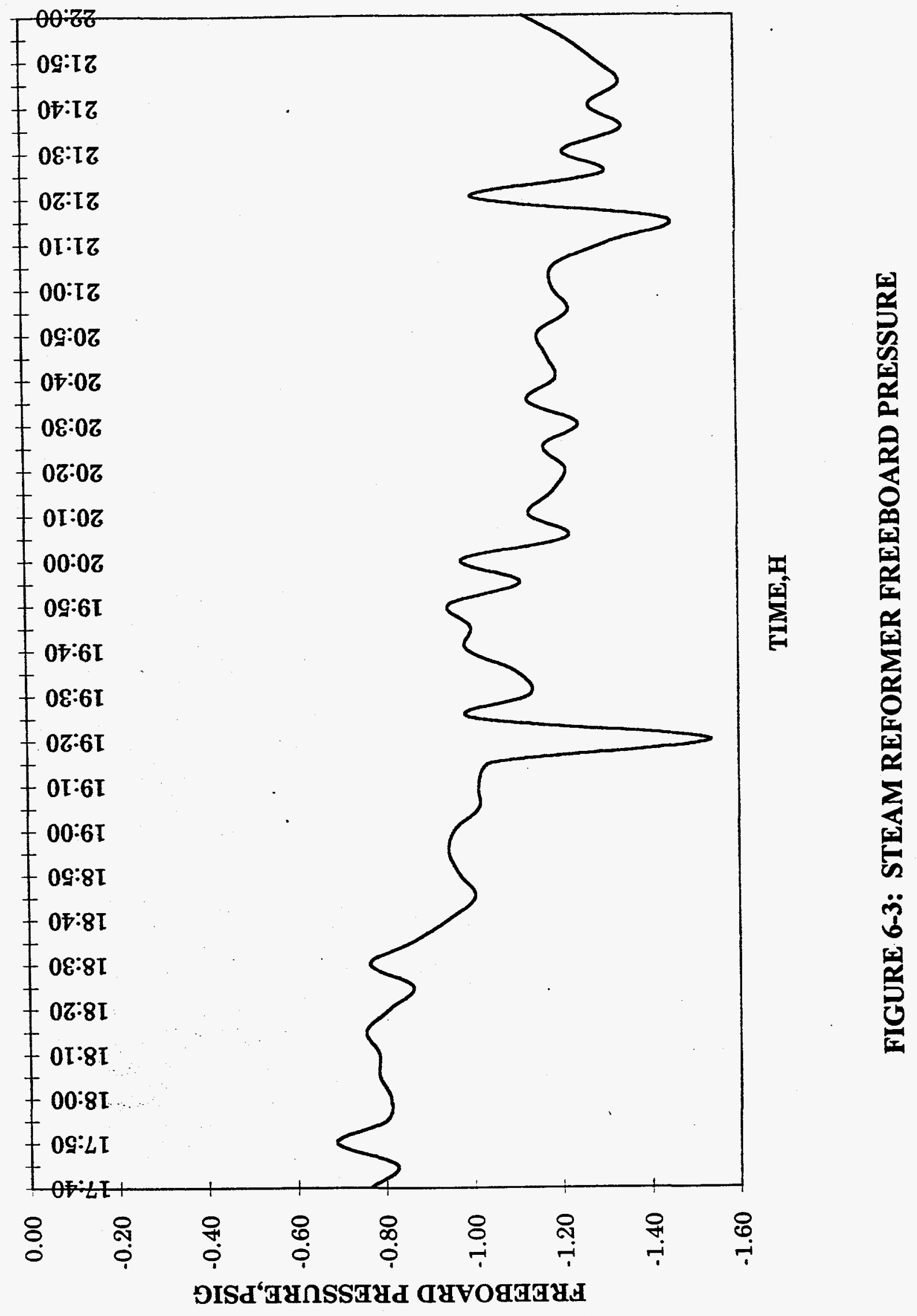




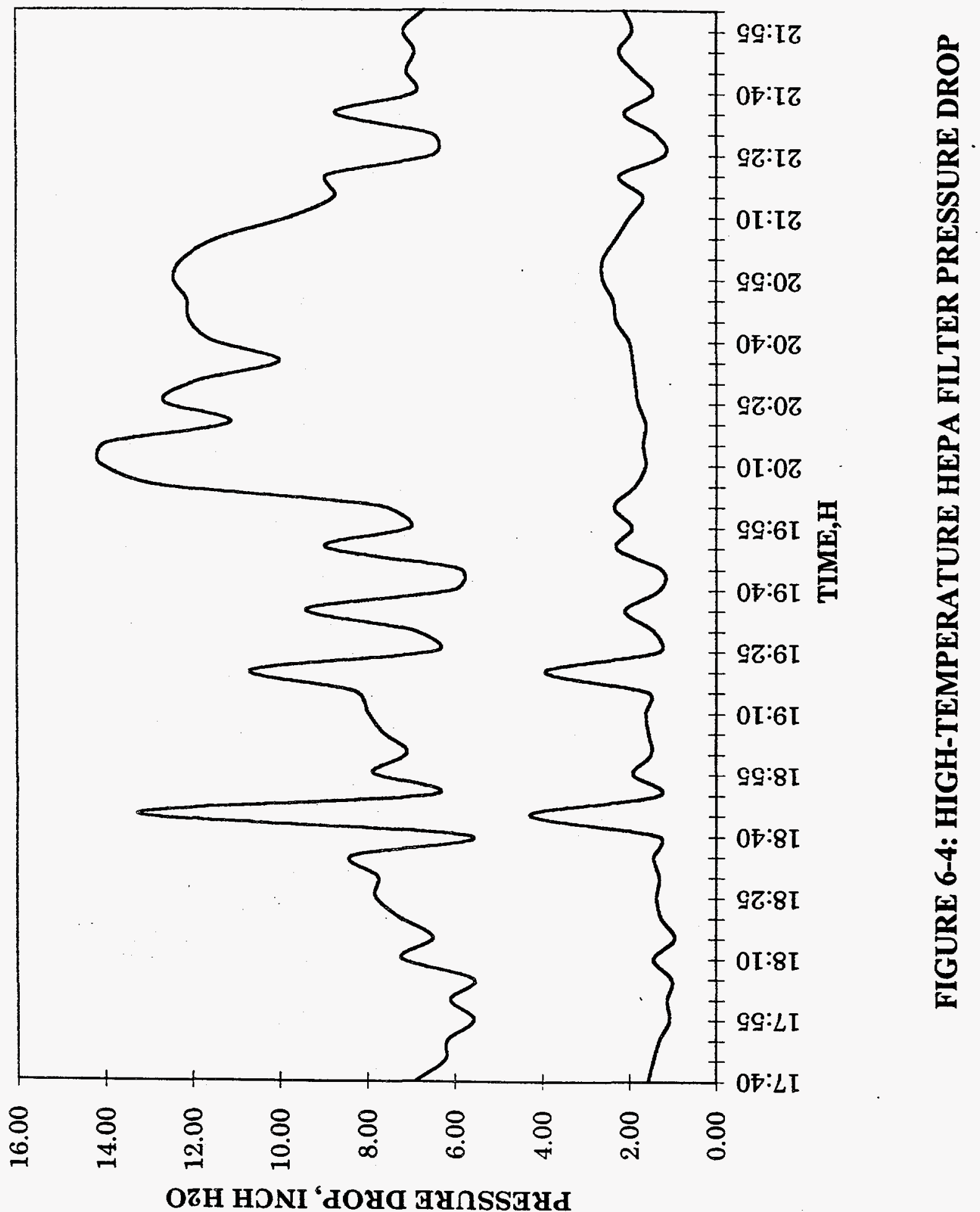



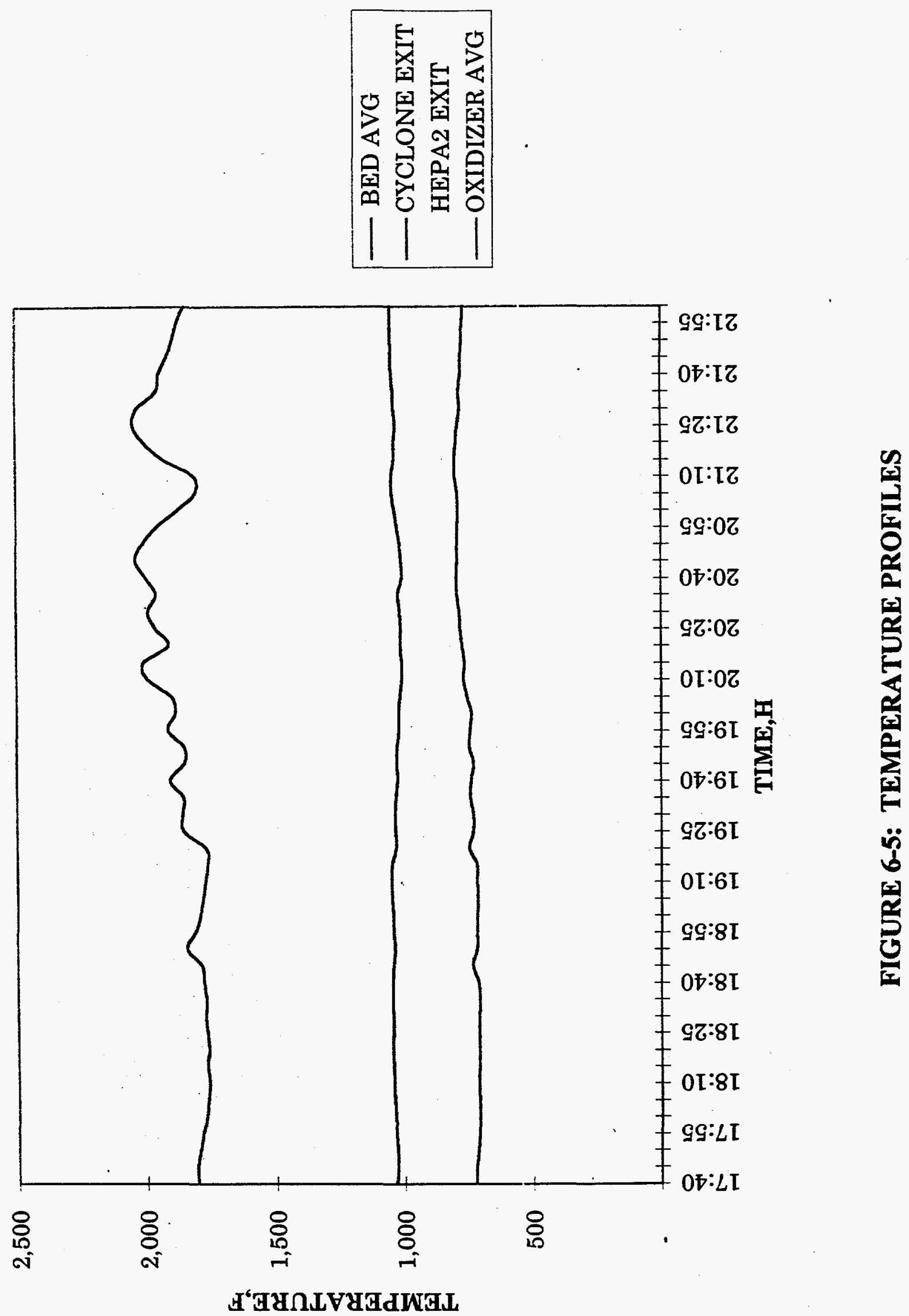


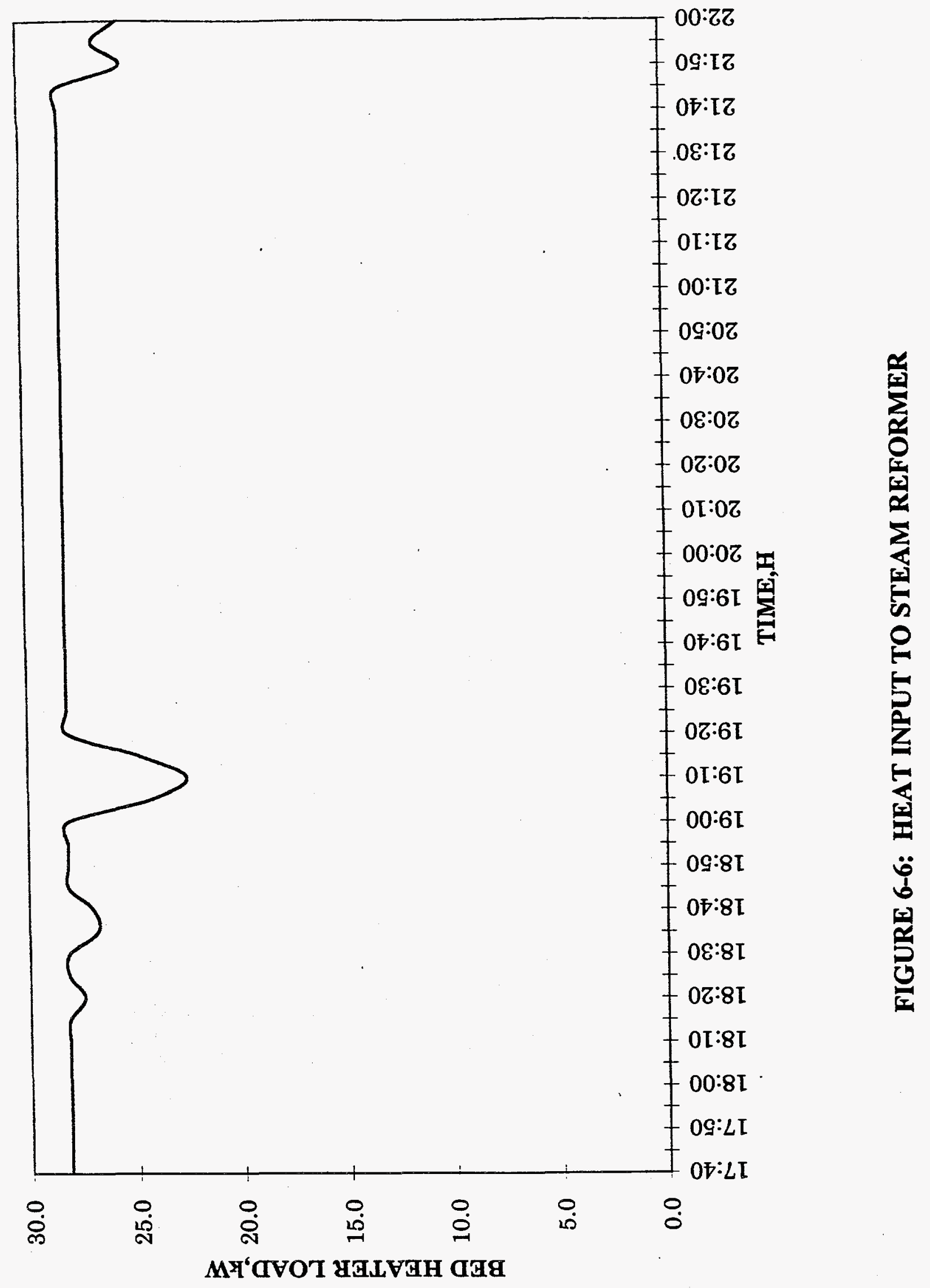


detection limit for demonstrating $99.9999 \%$ DRE. Therefore, additional tests were performed with higher concentrations of monochlorobenzene and/or cerium. These correspond to Tests 3, 4 and 5. A test (No. 6) was also performed by substituting ethylene glycol for monochlorobenzene.

The main performance parameters are listed in Tables 6-15 through $\underline{6-17}$. It is seen that the target DRE (99.9999\%) for monochlorobenzene has been achieved and that the cerium has almost completely (>99.9\%) been retained within the system. Table 6-18 furnishes data on the HT HEPA2 exit gas composition and Table 6-19 indicates stack flue gas composition.

- Emissions of PCDD/PCDF were measured simultaneously at the HT HEPA2 exit and the stack during Tests 1, 2, 4 and 5. The PCDD/PCDF emissions at the HT HEPA2 exit for Tests 1 and 2 are summarized in Tables 6-20 and 6-21. The PCDD/PCDF emissions at the stack and at HT HEPA2 exit for Tests 4 and 5 are given in Tables 6-22 and 6-23. Once again, the emissions results correspond to 2,3,7,8-TCDD toxicity equivalent basis. Stack samples were taken during Tests 1 and 2 but the samples were not analyzed for PCDD/PCDF due to a later (post Test 2) discovery of a problem with the thermal oxidizer. 

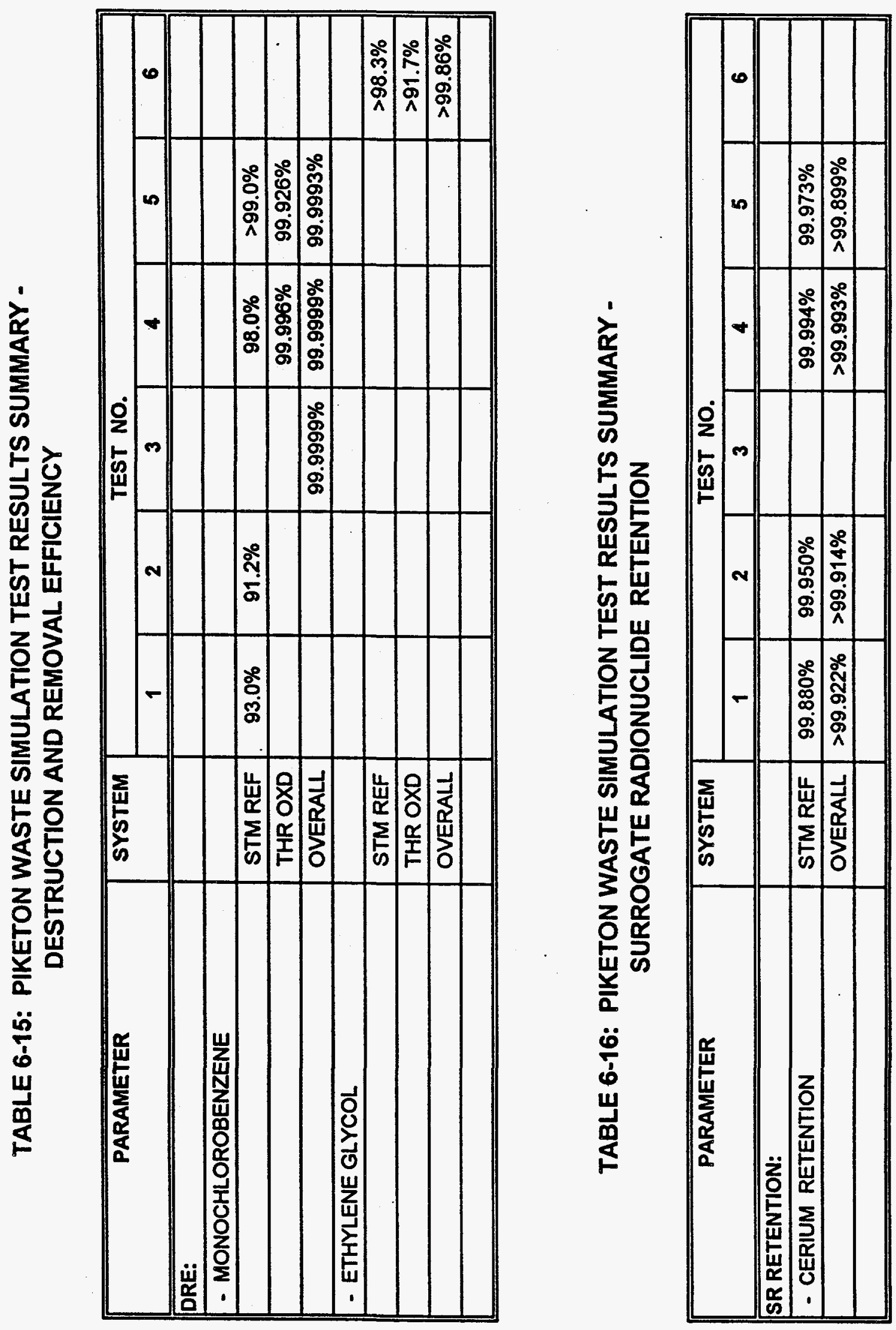


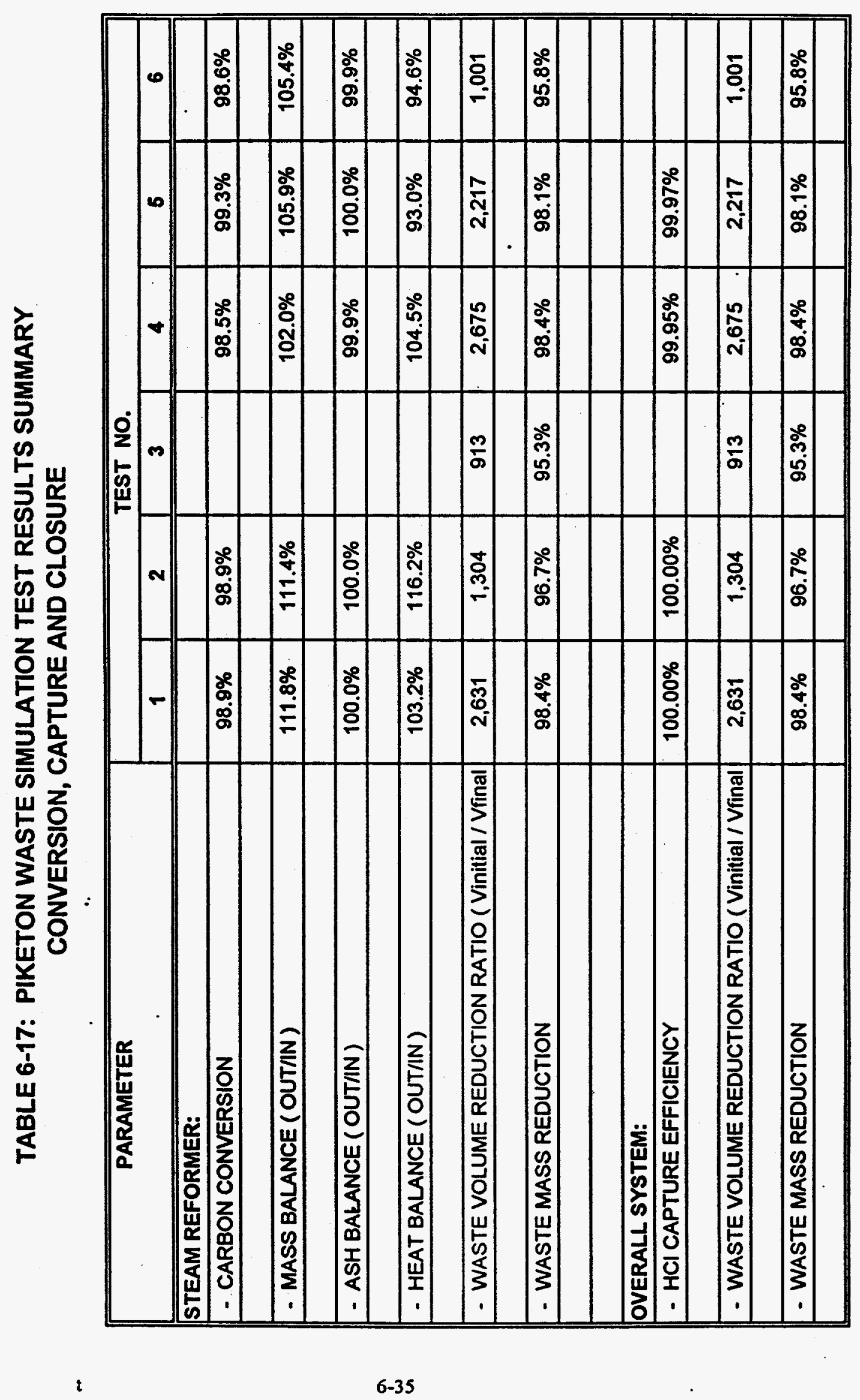




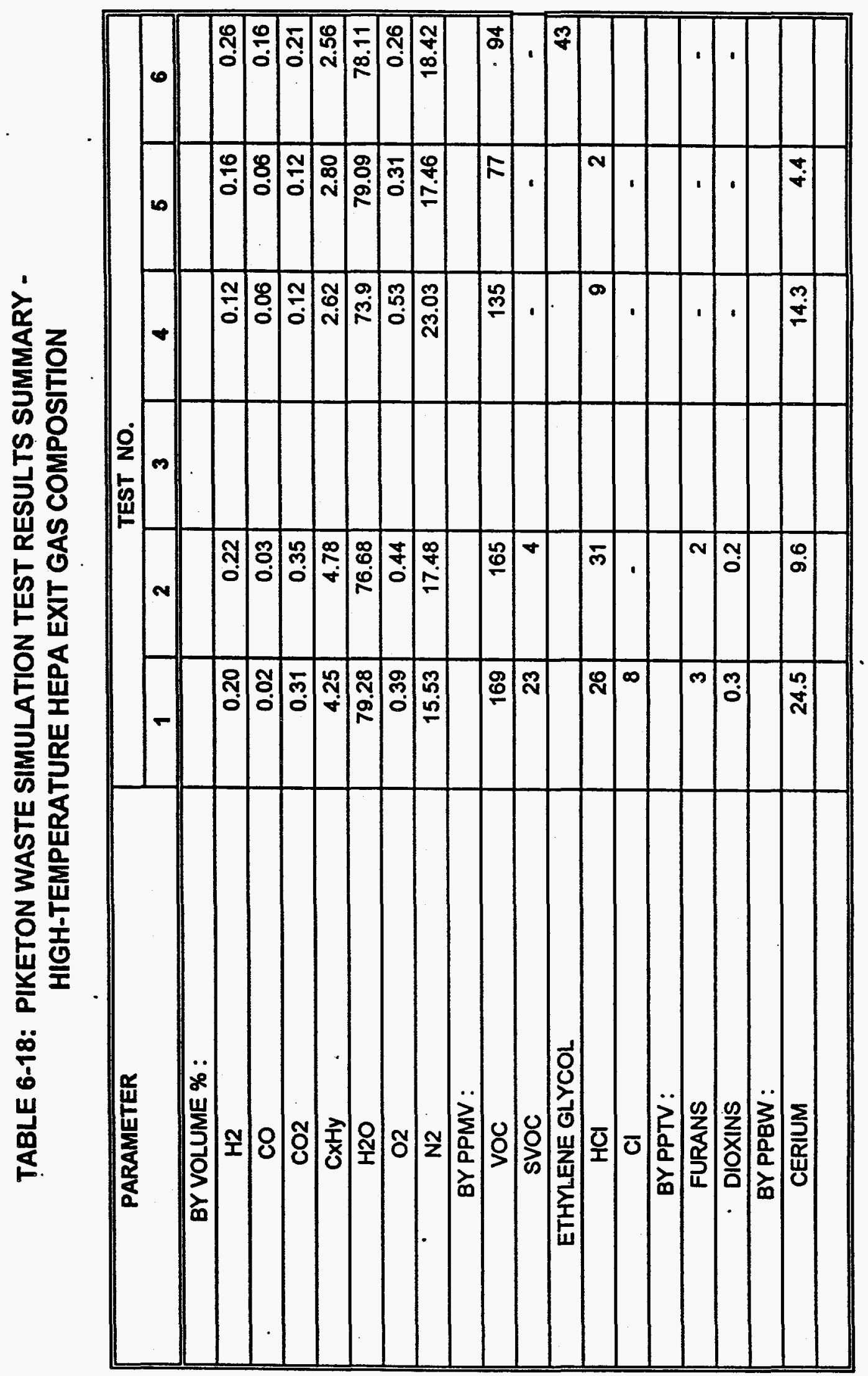




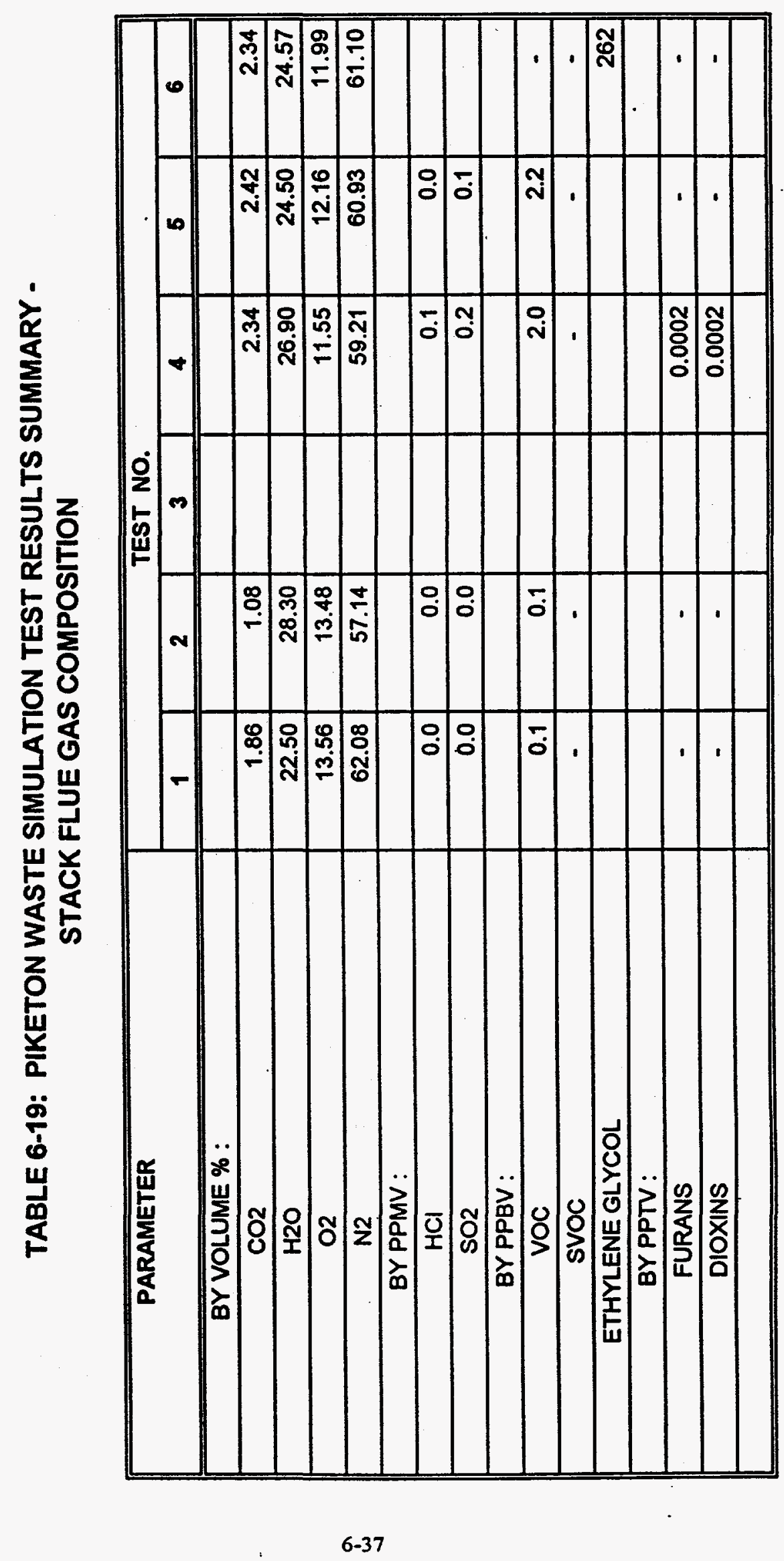




\section{TABLE 6-20: SUMMARY OF PCDD EMISSION RATES FOR PIKETON WASTE \\ TESTS 1 AND 2}

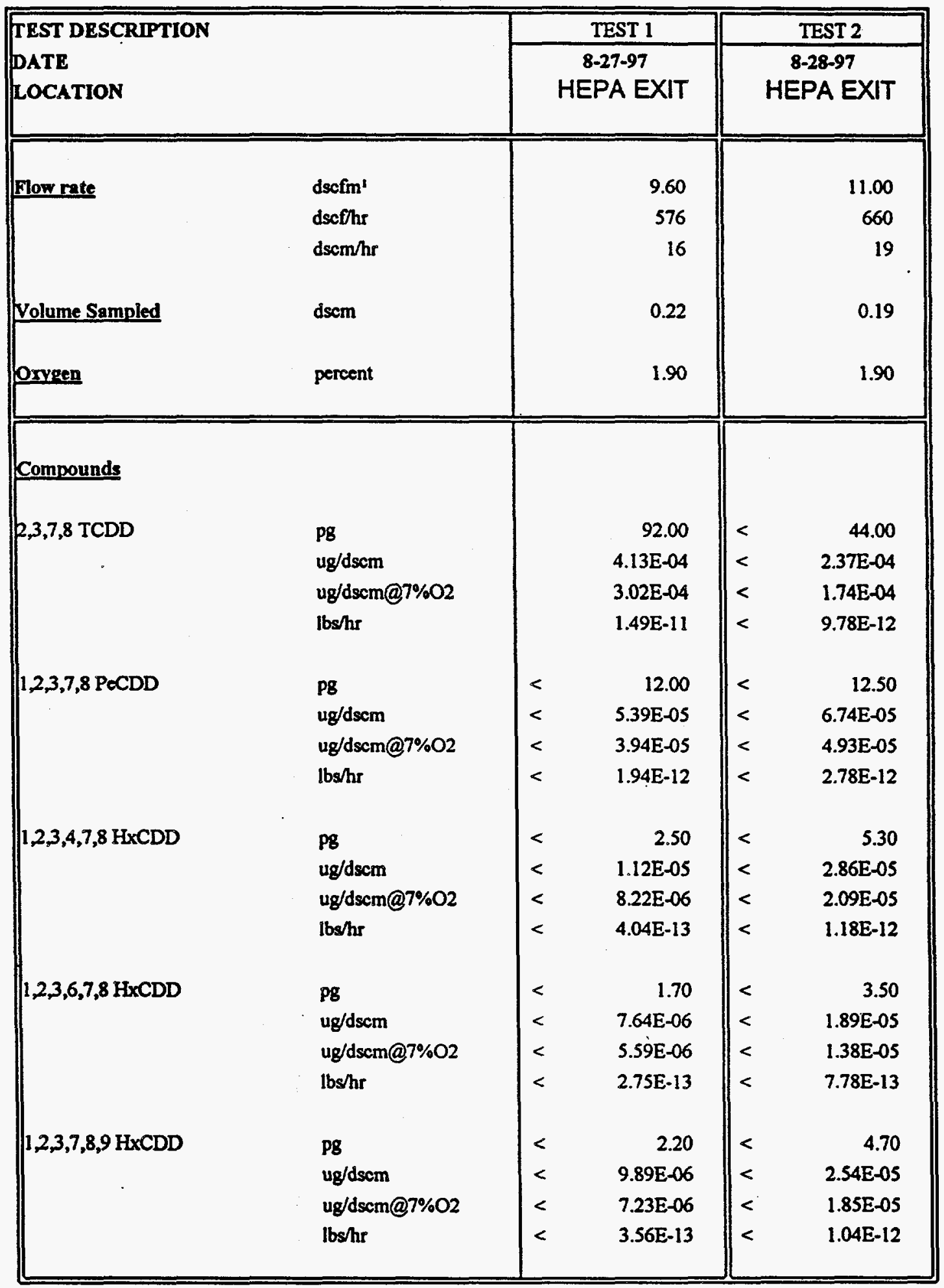




\section{TABLE 6-20: SUMMARY OF PCDD EMISSION RATES FOR PIKETON WASTE \\ TESTS 1 AND 2 \\ ( Continued )}

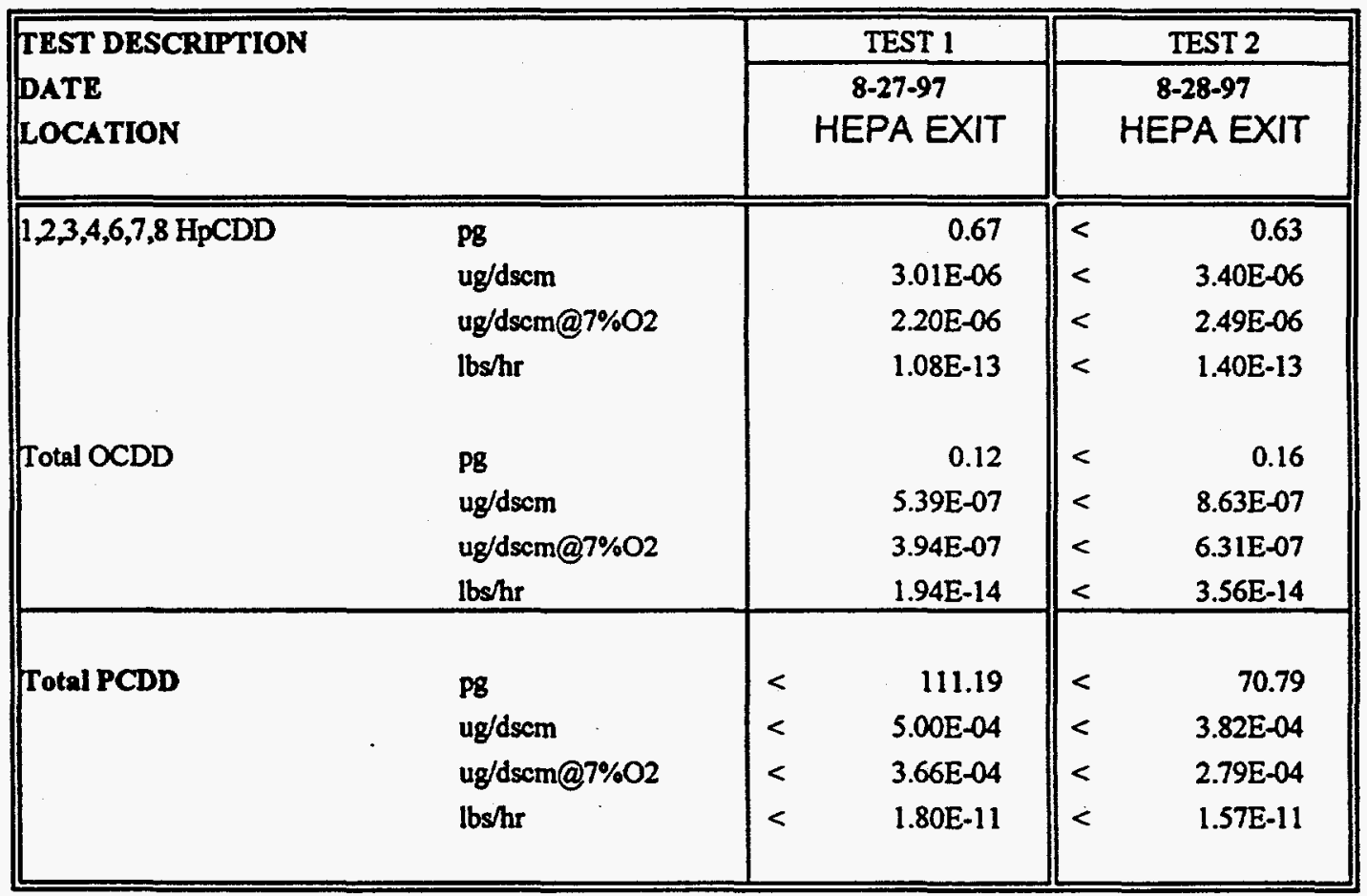

1 - Actual cubic feet per minute (acfm) was measured at the stack exhaust by method 1 and 2, but was not measured at HEPA Exit.

$2-\mathrm{dscfm}=\mathrm{dry}$ standard cubic feet per minute at $68^{\circ} \mathrm{F}$ and $29.92 \mathrm{in}$. $\mathrm{Hg}$.

3 - Dry gas flow rate at HEPA Exit was calculated from the gas moisture fraction measured with Method 0050 and the total steam input.

4- Oxygen concentration for August 27, 1997 was taken from August 28, 1997 data. 
TABLE 6-21: SUMMARY OF PCDF EMISSION RATES

FOR PIKETON WASTE

TESTS 1 AND 2

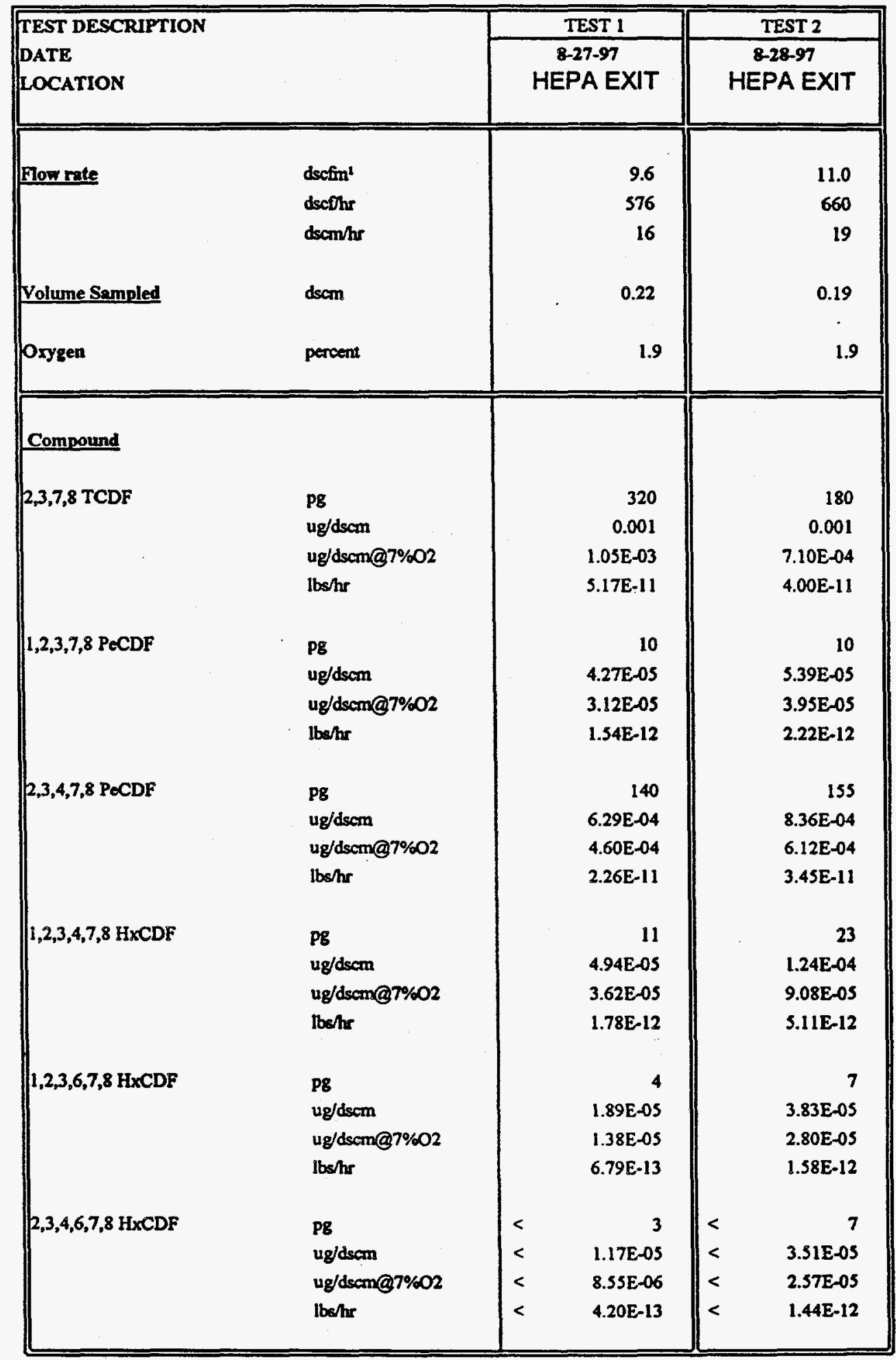


TABLE 6-21: SUMMARY OF PCDF EMISSION RATES

FOR PIKETON WASTE

TESTS 1 AND 2

(Continued)

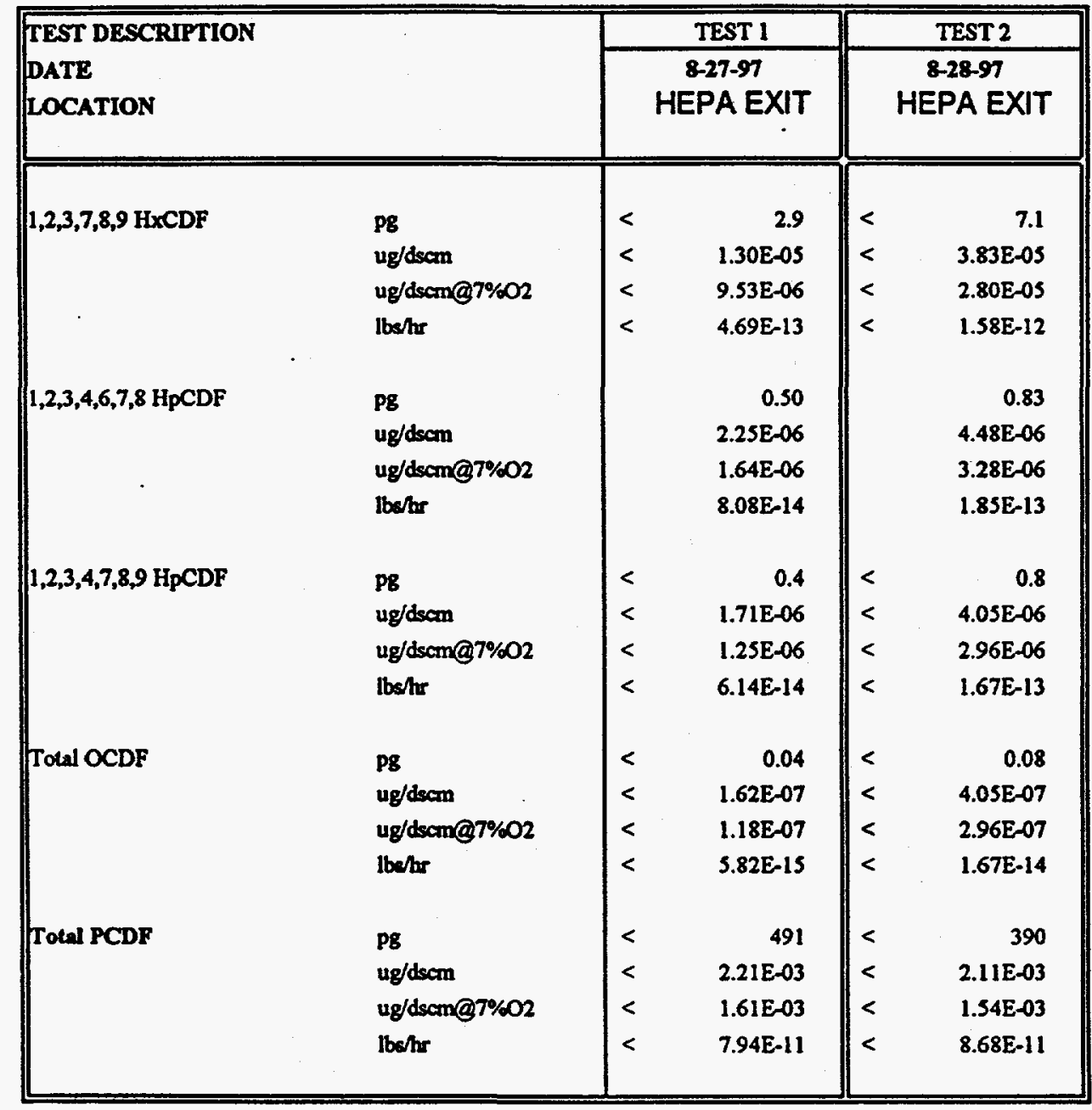

1 - Actual cubic feet per minute (acfin) was measured at the stack exhaust by method 1 and 2, but was not measured at HEPA Exit.

2 - dacin = dy andard cubic fect per minute at $68^{\circ} \mathrm{F}$ and $29.92 \mathrm{in}$. Hg

3 - Dry gas Elow rate at HEPA Exit was calculatod from the gas moisture fraction measured with Method OOSO and the total seam input.

4- Oxygen concentration for August 27, 1997 was taken from August 28, 1997 dati 
TABLE 6-22: SUMMARY OF PCDD EMISSION RATES

FOR PIKETON WASTE

TESTS 4 AND 5

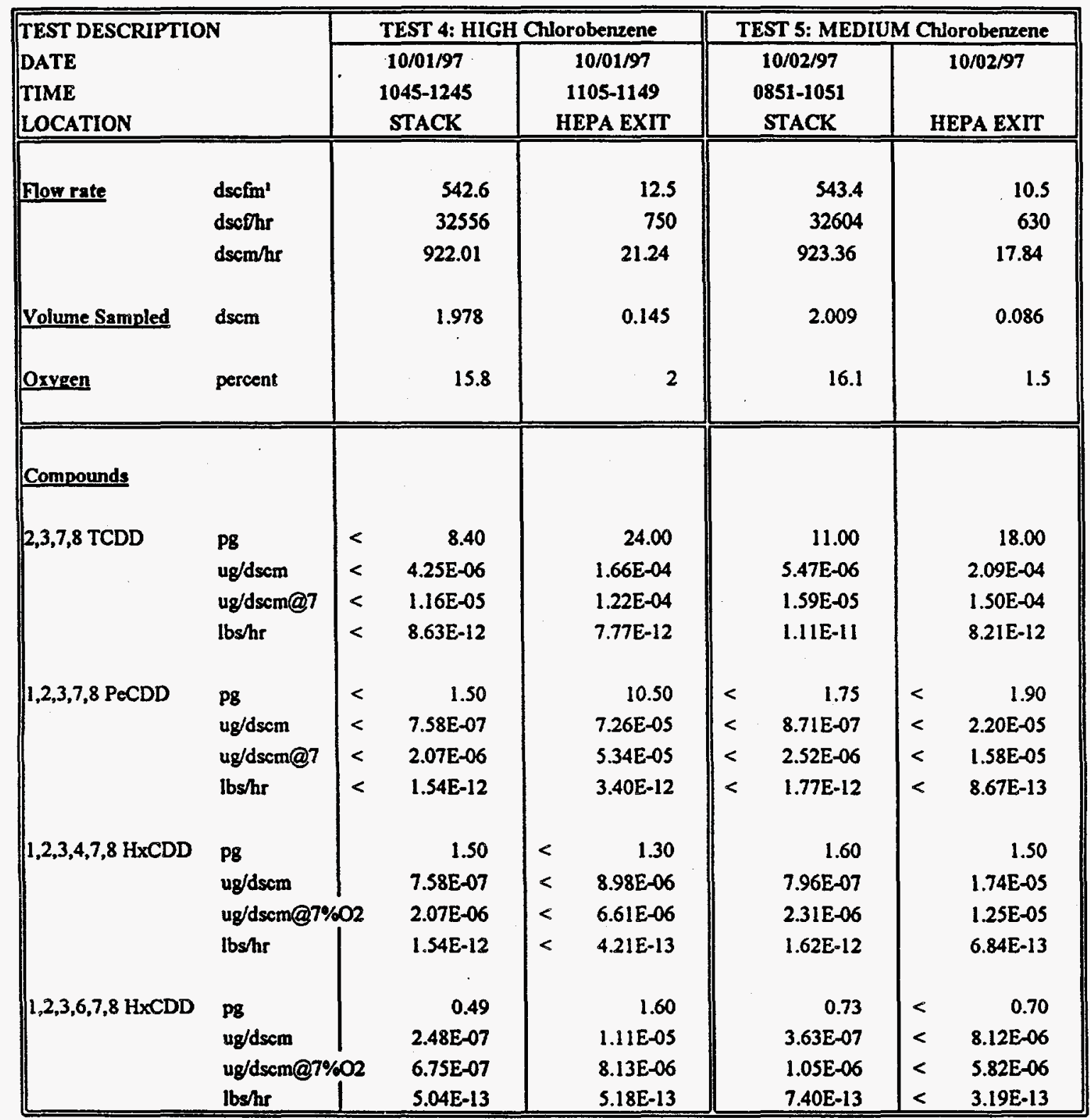


TABLE 6-22: SUMMARY OF PCDD EMISSION RATES

FOR PIKETON WASTE

TESTS 4 AND 5

(Continued)

\begin{tabular}{|c|c|c|c|c|c|}
\hline \multirow{2}{*}{\multicolumn{2}{|c|}{$\begin{array}{l}\text { TEST DESCRIPTION } \\
\text { DATE } \\
\text { TIME } \\
\text { LOCATION } \\
\end{array}$}} & \multicolumn{2}{|c|}{ TEST 4: HIGH Chlorobenzene } & \multicolumn{2}{|c|}{ TEST 5: MEDIUM Chlorobenzene } \\
\hline & & $\begin{array}{c}10 / 01 / 97 \\
1045-1245 \\
\text { STACK } \\
\end{array}$ & $\begin{array}{c}10 / 01 / 97 \\
1105-1149 \\
\text { HEPA EXIT } \\
\end{array}$ & $\begin{array}{c}10 / 02 / 97 \\
0851-1051 \\
\text { STACK } \\
\end{array}$ & $\begin{array}{r}\text { 10/02/97 } \\
\text { HEPA EXIT } \\
\end{array}$ \\
\hline $\begin{array}{l}1,2,3,7,8,9 \mathrm{HxCDD} \\
.\end{array}$ & $\begin{array}{l}\text { Pg } \\
\text { ug/dscm } \\
\text { ug/dscm@7 } \\
\text { lbs/hr }\end{array}$ & $\begin{array}{rr}< & 0.31 \\
< & 1.57 \mathrm{E}-07 \\
< & 4.27 \mathrm{E}-07 \\
< & 3.19 \mathrm{E}-13\end{array}$ & $\begin{array}{r}1.90 \\
1.31 \mathrm{E}-05 \\
9.66 \mathrm{E}-06 \\
6.15 \mathrm{E}-13\end{array}$ & $\begin{array}{r}0.40 \\
1.99 \mathrm{E}-07 \\
5.77 \mathrm{E}-07 \\
4.05 \mathrm{E}-13\end{array}$ & $\begin{array}{r}0.93 \\
1.08 \mathrm{E}-05 \\
7.73 \mathrm{E}-06 \\
4.24 \mathrm{E}-13\end{array}$ \\
\hline $1,2,3,4,6,7,8 \mathrm{HPCDD}$ & $\begin{array}{l}\text { Pg } \\
\text { ug/dscm } \\
\text { ug/dscm@7\%O } \\
\text { lbs/hr }\end{array}$ & $\begin{array}{r}0.72 \\
3.64 \mathrm{E}-07 \\
9.92 \mathrm{E}-07 \\
7.40 \mathrm{E}-13\end{array}$ & $\begin{array}{r}1.50 \\
1.04 \mathrm{E}-05 \\
7.62 \mathrm{E}-06 \\
4.85 \mathrm{E}-13\end{array}$ & $\begin{array}{r}0.78 \\
3.88 \mathrm{E}-07 \\
1.12 \mathrm{E}-06 \\
7.90 \mathrm{E}-13\end{array}$ & $\begin{array}{r}0.94 \\
1.09 \mathrm{E}-05 \\
7.81 \mathrm{E}-06 \\
4.29 \mathrm{E}-13\end{array}$ \\
\hline Total OCDD & $\begin{array}{l}\text { Pg } \\
\text { ug/dscm } \\
\text { ug/dscm@7\%O } \\
\text { lbs/hr }\end{array}$ & $\begin{array}{r}0.36 \\
1.82 \mathrm{E}-07 \\
4.96 \mathrm{E}-07 \\
3.70 \mathrm{E}-13\end{array}$ & $\begin{array}{r}1.20 \\
8.29 \mathrm{E}-06 \\
6.10 \mathrm{E}-06 \\
3.88 \mathrm{E}-13 \\
\end{array}$ & $\begin{array}{r}0.46 \\
2.29 E-07 \\
6.63 E-07 \\
4.66 E-13\end{array}$ & $\begin{array}{r}0.54 \\
6.26 \mathrm{E}-06 \\
4.49 \mathrm{E}-06 \\
2.46 \mathrm{E}-13\end{array}$ \\
\hline Total PCDD & $\begin{array}{l}\text { pg } \\
\text { ug/dscm } \\
\text { ug/dscm@7 } \\
\text { lbs/hr }\end{array}$ & $\begin{array}{r}13.28 \\
6.71 \mathrm{E}-06 \\
1.83 \mathrm{E}-05 \\
1.36 \mathrm{E}-11\end{array}$ & $\begin{array}{r}42.00 \\
2.90 \mathrm{E}-04 \\
2.13 \mathrm{E}-04 \\
1.36 \mathrm{E}-11\end{array}$ & $\begin{array}{r}16.72 \\
8.32 E-06 \\
2.41 E-05 \\
1.69 E-11\end{array}$ & $\begin{array}{rr}< & 24.51 \\
< & 2.84 \mathrm{E}-04 \\
< & 2.04 \mathrm{E}-04 \\
< & 1.12 \mathrm{E}-11\end{array}$ \\
\hline
\end{tabular}

1 - Actual cubic feet per minute (acfm) was measured at the stack exhaust by method 1 and 2 , but was not measured at HEPA exit.

$2-\mathrm{dscfm}=\mathrm{dry}$ standard cubic feet per minute at $68^{\circ} \mathrm{F}$ and $29.92 \mathrm{in} . \mathrm{Hg}$.

3 - Dry gas flow rate at HEPA Exit was calculated from the gas moisture fraction measured with Method 0050 and the total steam input. 
TABLE 6-23: SUMMARY OF PCDF EMISSION RATES

FOR PIKETON WASTE

TESTS 4 AND 5

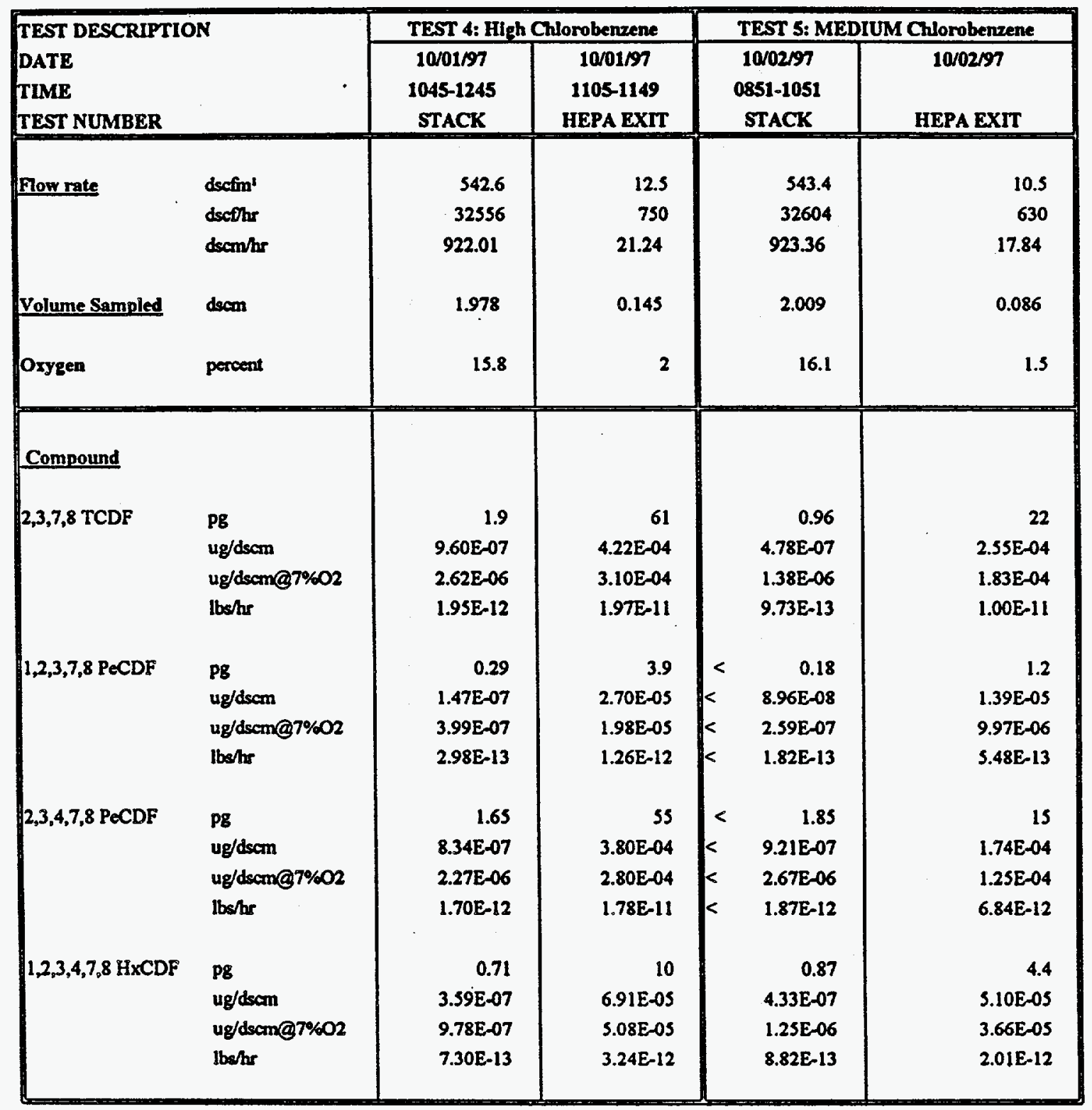


TABLE 6-23: SUMMARY OF PCDF EMISSION RATES

FOR PIKETON WASTE

TESTS 4 AND 5

(Continued)

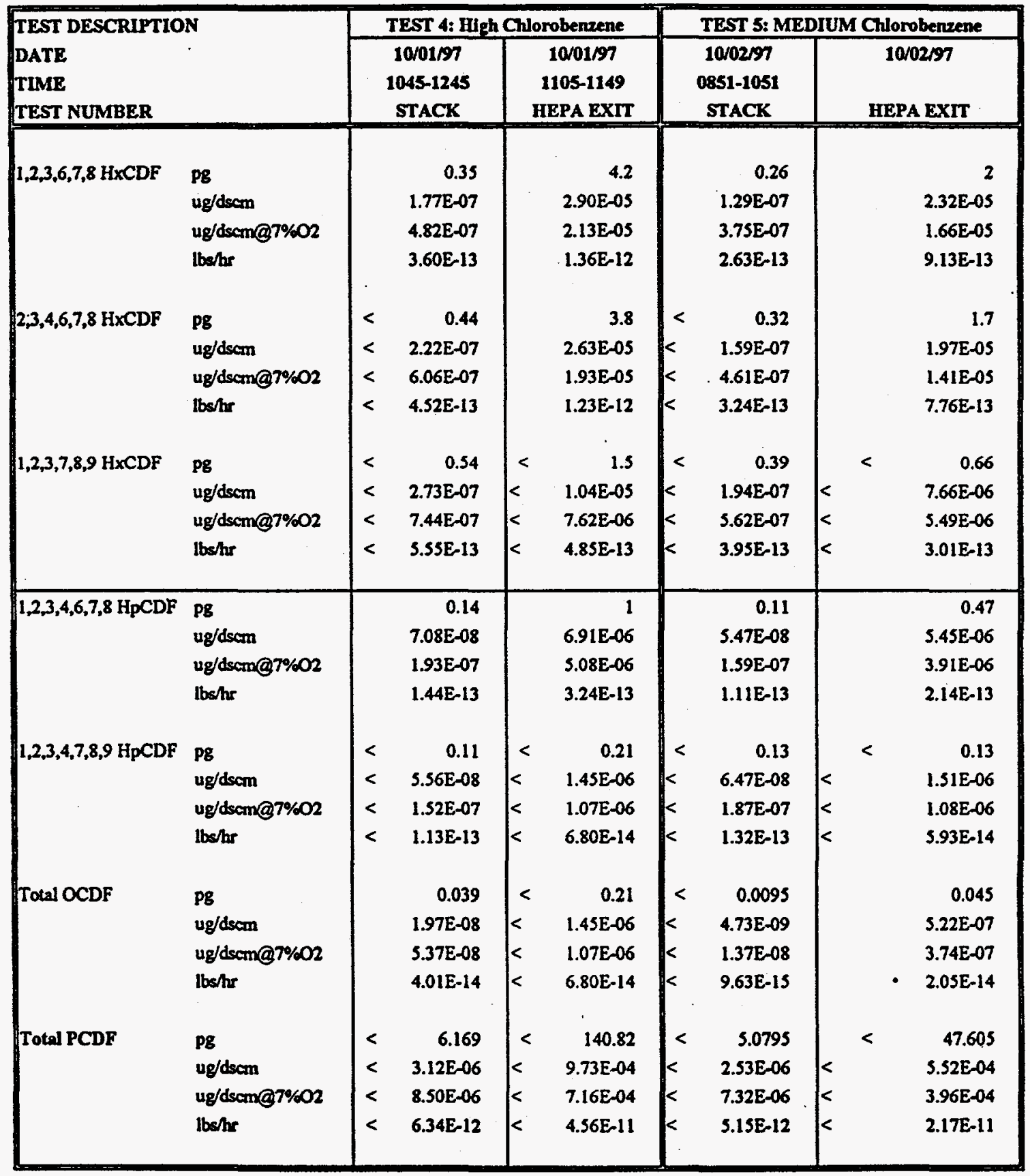

1 - Actual cubic foet per minute (acfin) was measured at the stack exhaust by EPA Method 1 and 2, but was not measured at the HEPA Exit.

2 - dectin = dry standard cubic feet per Minute at $68^{\circ} \mathrm{F}$ and $29.92 \mathrm{in.} \mathrm{Hg}$

3- Dry gas flow rate at HEPA Exit was calculated from the gas moisture fraction measured with Method 0050 and the total steam input. 
The operating parameter trends for one of the six test periods are shown in Figures 6-7 through $\underline{6-11}$ (the remaining charts are included as Appendix $D$ ). The following five parameters are included for each test:

\footnotetext{
- Steam reformer fluidization flow;

- $\quad$ Steam reformer freeboard pressure;

- HT HEPA1 and HT HEPA2 pressure drops;

- $\quad$ Temperature profiles (bed average, cyclone exit, HT HEPA2 exit and thermal oxidizer average temperatures); and

- Electrical heat input to the steam reformer.
}

Multiple Organic Feedstock Retests. The overall DRE values for some of the Principal Organic Hazardous Constituents (POHC) turned out to be slightly below the target goal of $99.99 \%$ in the case of the multiple surrogate tests (see Table 6-6). This shortcoming was subsequently traced to the inferior performance of the thermal oxidizer. The problem was then corrected during the Piketon waste simulation tests and $99.9999 \%$ DRE was obtained for monochlorobenzene - a PCB surrogate. These retests were performed to confirm $>99.99 \%$ DRE for the POHCs in the multiple surrogates. Three tests were performed with the three different organic formulations listed in Table 6-2.

Three sets of stack samples were taken during each retest and the DRE values are given in Table 6-24. The PCDD/PCDF emissions at the stack were measured during Test R3 and the results are summarized in Tables 6-25 and 6-26. The feedstock for Tests R1 and R2 did not contain any chlorine and therefore, PCDD/PCDF samples were not taken during these two tests. The emissions reported in Tables 6-25 and 6-26 correspond to 2,3,7,8-TCDD toxicity equivalent basis.

The stack PCDD and PCDF emissions were converted using toxic equivalent factors (TEF) to $2,3,6,8$ - TCDD TEQ. These values for selected multiple surrogate, Piketon waste and multiple organic feedstock tests are given in Table 6-27. 


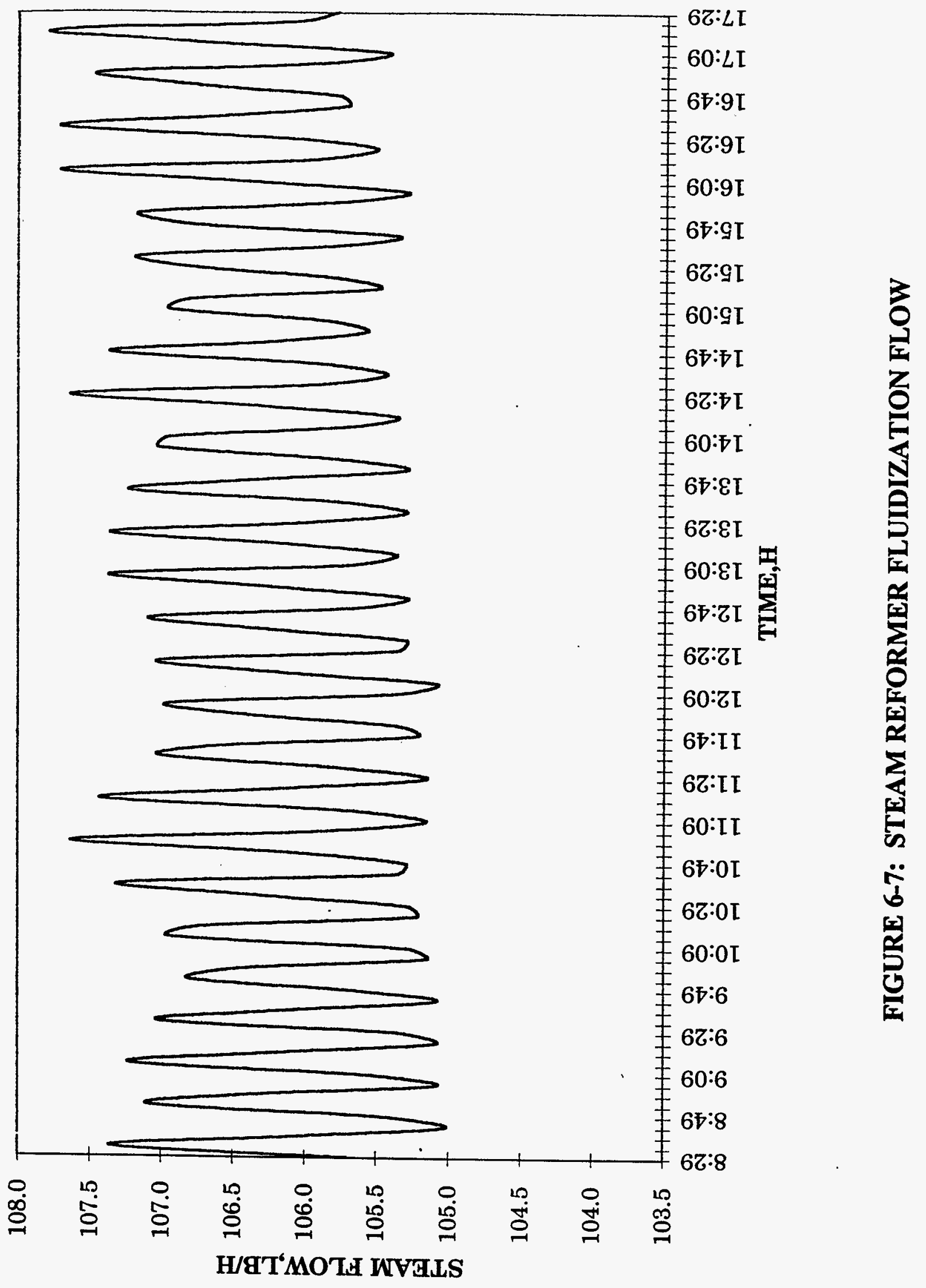




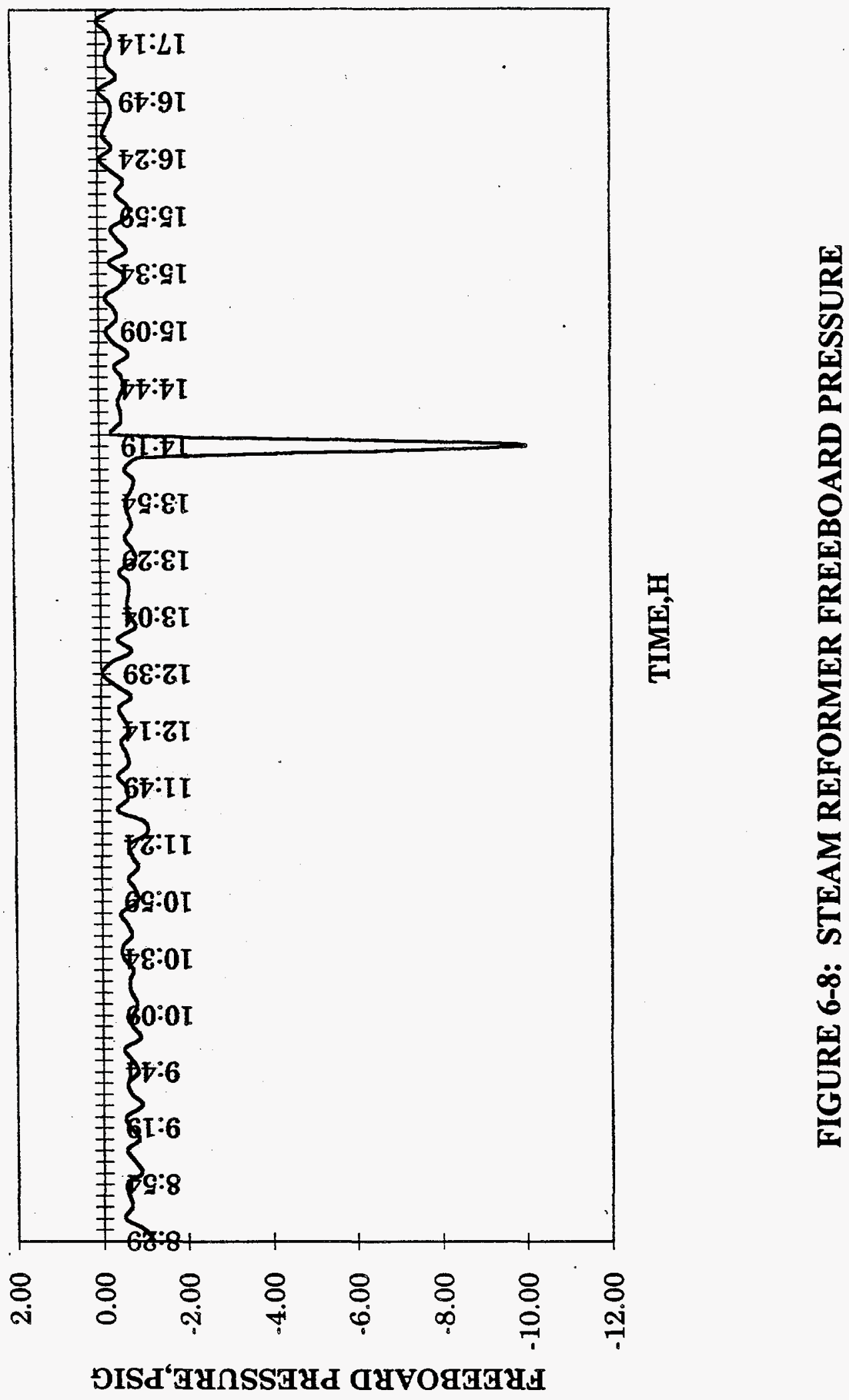




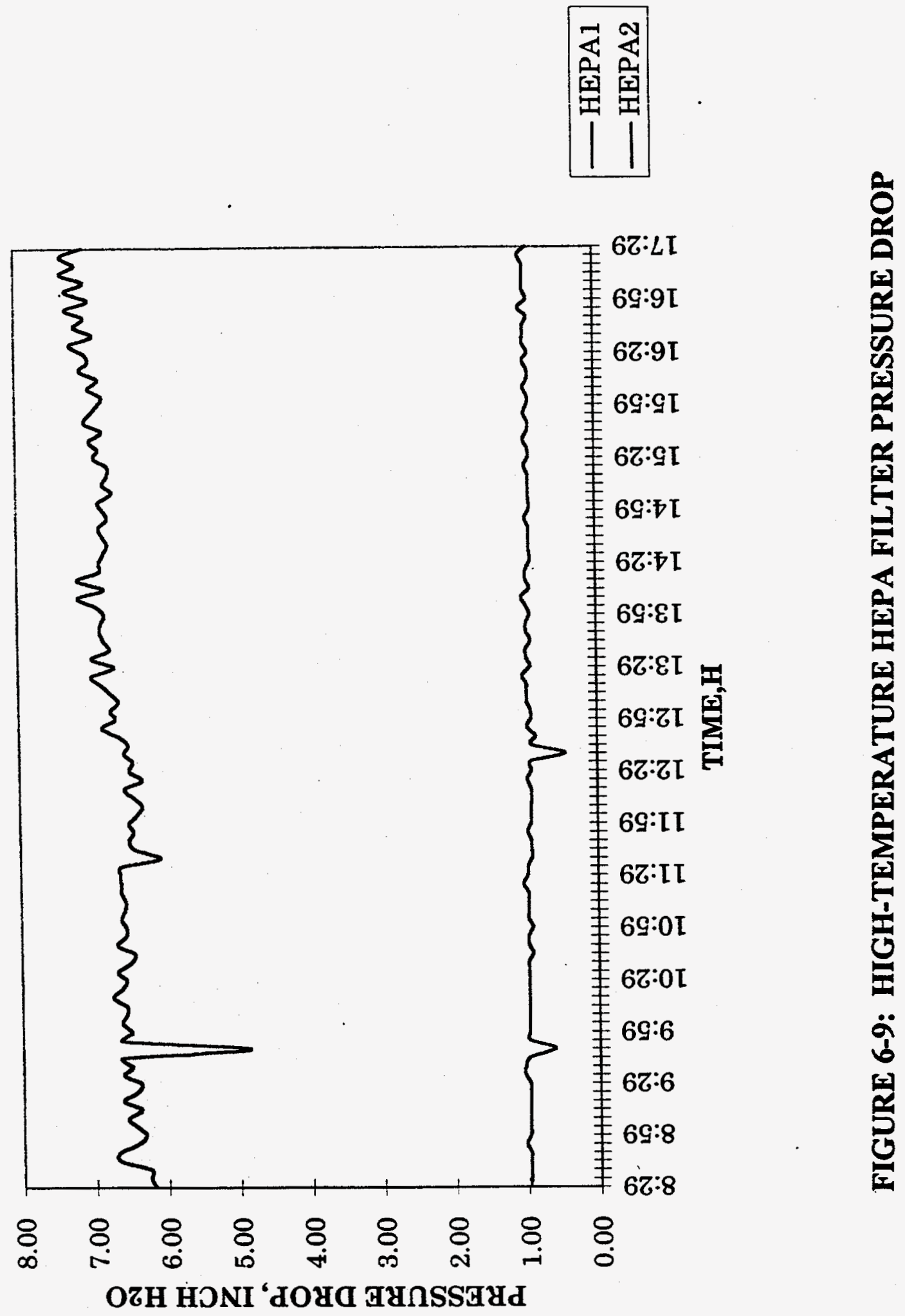



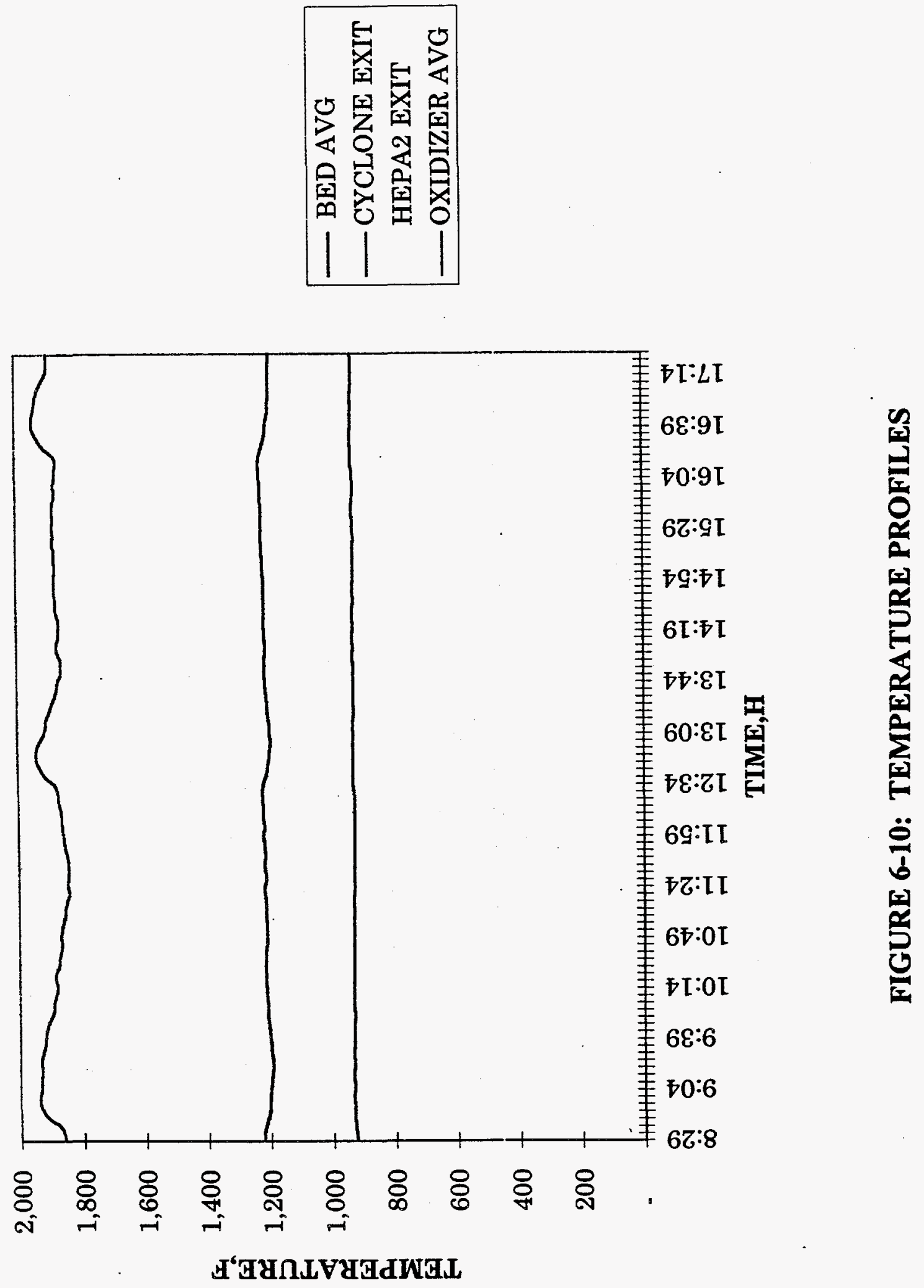


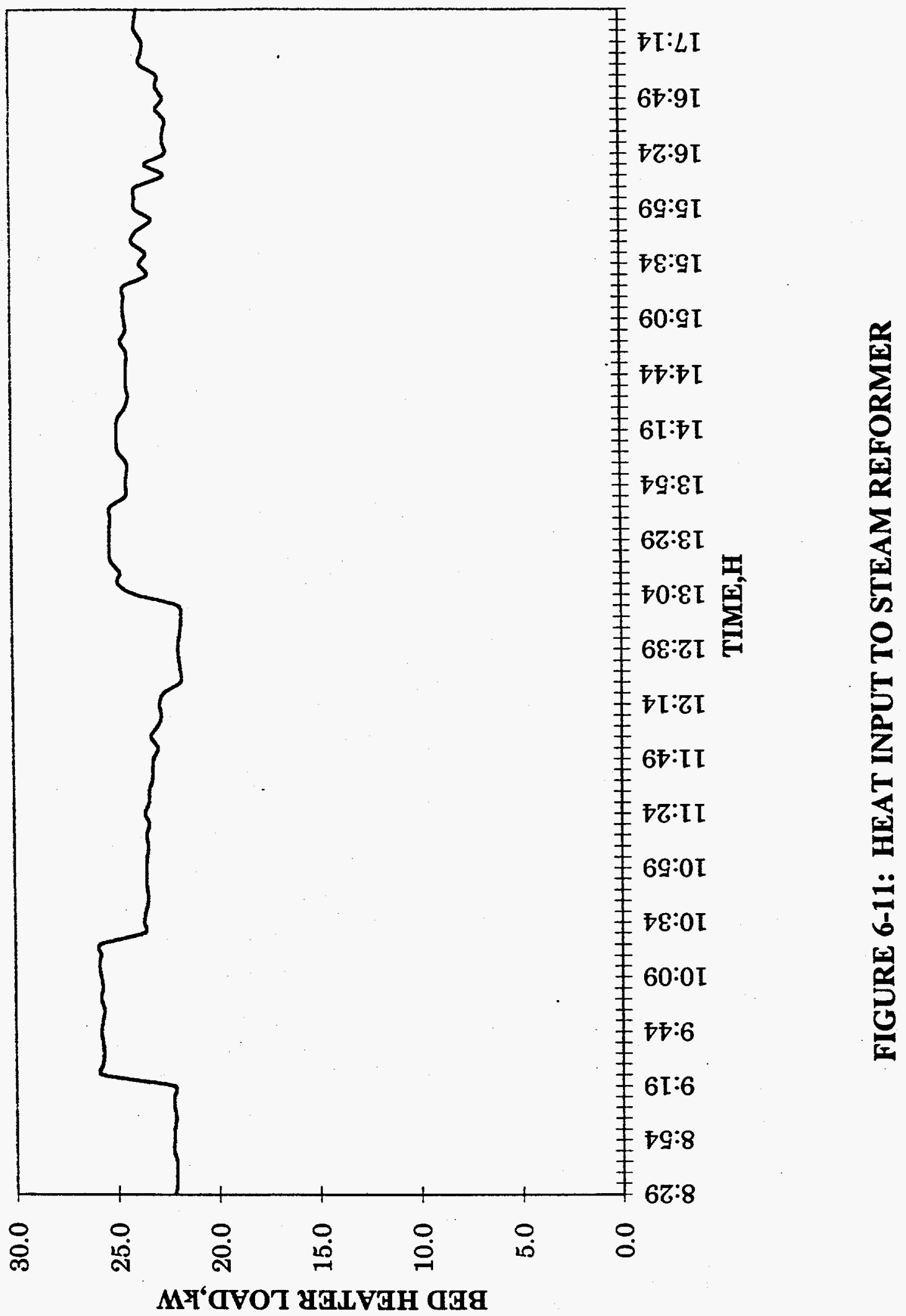


TABLE 6-24: MULTIPLE ORGANIC FEEDSTOCK RETEST RESULTS SUMMARY - DESTRUCTION AND REMOVAL EFFICIENCY

\begin{tabular}{||l|c|c|c|c||}
\hline \multirow{2}{*}{ PARAMETER } & \multirow{2}{*}{ SAMPLE } & \multicolumn{3}{|c|}{ TEST NO. } \\
\cline { 3 - 5 } & & R1 & R2 & R3 \\
\hline OVERALL DRE: & & & & \\
\hline ETHYLENE GLYCOL & 1 & $>99.996 \%$ & & \\
\hline & 2 & $>99.996 \%$ & & \\
\hline & 3 & $>99.996 \%$ & & \\
\hline- NAPHTHALENE & & & & \\
\hline & 1 & & $99.9999 \%$ & \\
\hline & 2 & & $99.9999 \%$ & \\
\hline & 3 & & $99.9999 \%$ & \\
\hline- TOLUENE & & & & \\
\hline & 1 & & & $>99.99 \%$ \\
\hline & 2 & & & $>99.998 \%$ \\
\hline & 3 & & & $>99.999 \%$ \\
\hline$-1,2$-DICHLOROBENZENE & 1 & & & $99.9998 \%$ \\
\hline & 2 & & & $99.9999 \%$ \\
\hline & 3 & & & $99.9999 \%$ \\
\hline & & & & \\
\hline- TETRACHLOROETHYLENE & 1 & & & $99.9994 \%$ \\
\hline & 2 & & & $99.9996 \%$ \\
\hline & 3 & & & $99.9998 \%$ \\
\hline & & & & \\
\hline
\end{tabular}


TABLE 6-25: SUMMARY OF PCDD EMISSION RATES

FOR MULTIPLE ORGANIC FEEDSTOCK

TEST R3

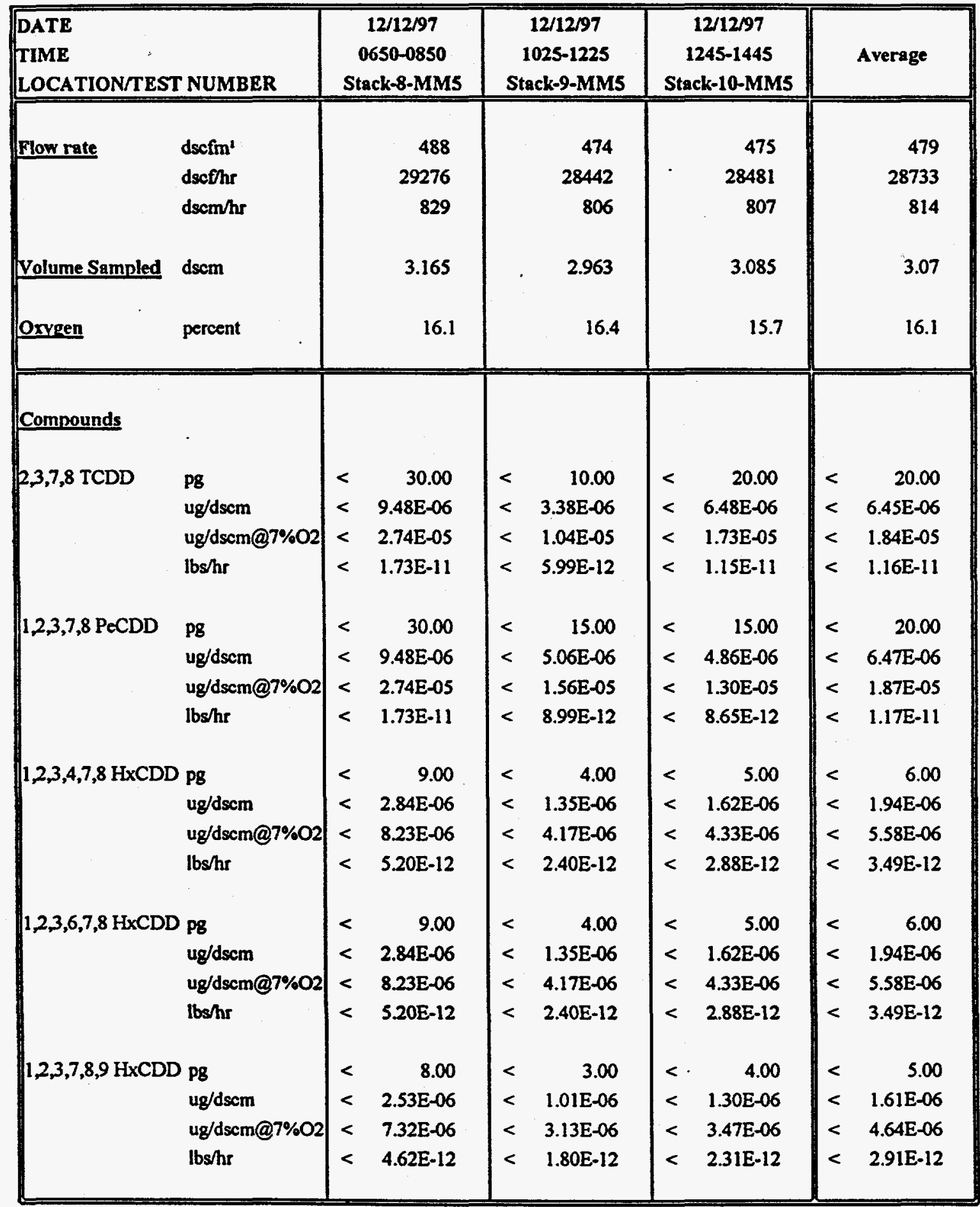


TABLE 6-25: SUMMARY OF PCDD EMISSION RATES FOR MULTIPLE ORGANIC FEEDSTOCK

TEST R3

(Continued)

\begin{tabular}{|c|c|c|c|c|c|}
\hline \multicolumn{2}{|c|}{$\begin{array}{l}\text { DATE } \\
\text { TIME } \\
\text { LOCATION/TEST NUMBER } \\
\end{array}$} & $\begin{array}{c}12 / 12 / 97 \\
0650-0850 \\
\text { Stack-8-MM5 } \\
\end{array}$ & $\begin{array}{c}12 / 12 / 97 \\
1025-1225 \\
\text { Stack-9-MMS } \\
\end{array}$ & $\begin{array}{c}12 / 12 / 97 \\
1245-1445 \\
\text { Stack-10-MM5 } \\
\end{array}$ & Average \\
\hline \multicolumn{2}{|c|}{$1,2,3,4,6,7,8 \mathrm{HpCD}$ pg } & $<\quad 1.20$ & 0.40 & 0.60 & 0.73 \\
\hline & ug/dscm & $<\quad 3.79 \mathrm{E}-07$ & $<\quad 1.35 \mathrm{E}-07$ & $<\quad 1.94 \mathrm{E}-07$ & $<\quad 2.36 \mathrm{E}-07$ \\
\hline & ug/dscm@7\%02 & $<\quad 1.10 \mathrm{E}-06$ & $<\quad 4.17 \mathrm{E}-07$ & $<\quad 5.20 \mathrm{E}-07$ & $6.78 \mathrm{E}-07$ \\
\hline & $\mathrm{lbs} / \mathrm{hr}$ & $<\quad 6.93 \mathrm{E}-13$ & $<\quad 2.40 \mathrm{E}-13$ & $<\quad 3.46 \mathrm{E}-13$ & $4.26 \mathrm{E}-13$ \\
\hline \multirow[t]{4}{*}{ Total OCDD } & Pg & 0.35 & 0.18 & 0.23 & 0.25 \\
\hline & $\mathrm{ug} / \mathrm{dscm}$ & $1.11 E-07$ & $6.08 \mathrm{E}-08$ & 7.45E-08 & 8.20E-08 \\
\hline & ug/dscm@7\%02 & $3.20 \mathrm{E}-07$ & $1.88 \mathrm{E}-07$ & $1.99 \mathrm{E}-07$ & $2.36 \mathrm{E}-07$ \\
\hline & $\mathrm{lbs} / \mathrm{hr}$ & $2.02 \mathrm{E}-13$ & $1.08 \mathrm{E}-13$ & $1.33 \mathrm{E}-13$ & $1.48 \mathrm{E}-13$ \\
\hline \multirow[t]{4}{*}{ Total PCDD } & Pg & 87.60 & 36.60 & 49.80 & 58.00 \\
\hline & $\mathrm{ug} / \mathrm{dscm}$ & $2.77 E-05$ & $1.24 \mathrm{E}-05$ & $1.61 \mathrm{E}-05$ & $1.87 \mathrm{E}-05$ \\
\hline & ug/dscm@7\%O2 & $<\quad 8.01 \mathrm{E}-05$ & $<3.82 \mathrm{E}-05$ & 4.31E-05 & $5.38 \mathrm{E}-05$ \\
\hline & $\mathrm{lbs} / \mathrm{hr}$ & $<\quad 5.06 \mathrm{E}-11$ & $<\quad 2.19 \mathrm{E}-11$ & $<\quad 2.87 \mathrm{E}-11$ & $<\quad 3.37 \mathrm{E}-11$ \\
\hline
\end{tabular}

1 - $\mathrm{dscfm}=$ dry standard cubic feet per minute at $68^{\circ} \mathrm{F}$ and $29.92 \mathrm{in} . \mathrm{Hg}$. 
TABLE 6-26: SUMMARY OF PCDF EMISSION RATES

FOR MULTIPLE ORGANIC FEEDSTOCK

TEST R3

\begin{tabular}{|c|c|c|c|c|c|}
\hline \begin{tabular}{|l} 
DATE \\
TIME \\
LOCATION/TES \\
\end{tabular} & T NUMBER & $\begin{array}{c}12 / 12 / 97 \\
0650-0850 \\
\text { Stack-8-MM5 } \\
\end{array}$ & $\begin{array}{c}12 / 12 / 97 \\
1025-1225 \\
\text { Stack-9-MM5 }\end{array}$ & $\begin{array}{c}12 / 12 / 97 \\
1245-1445 \\
\text { Stack-10-MM5 }\end{array}$ & Average \\
\hline Flow rate & $\begin{array}{l}\text { dscfm } \\
\text { dscf/hr } \\
\text { dscm/hr }\end{array}$ & $\begin{array}{r}806 \\
48357 \\
1369\end{array}$ & $\begin{array}{r}811 \\
48644 \\
1378\end{array}$ & $\begin{array}{r}810 \\
48571 \\
1376\end{array}$ & $\begin{array}{r}809 \\
48524 \\
1374\end{array}$ \\
\hline $\begin{array}{l}\text { Volume Sampled } \\
\text { Oxygen }\end{array}$ & $\begin{array}{l}\text { dsem } \\
\text { percent }\end{array}$ & $\begin{array}{r}2.305 \\
2.5\end{array}$ & $\begin{array}{r}2.300 \\
2.4\end{array}$ & $\begin{array}{r}2.302 \\
3\end{array}$ & $\begin{array}{l}2 \\
3\end{array}$ \\
\hline Compounds & & & & & \\
\hline $2,3,7,8 \mathrm{TCDF}$ & $\begin{array}{l}\mathrm{pg} \\
\text { ug/dscm } \\
\text { ug/dscm@7\%02 } \\
\mathrm{lbs} / \mathrm{hr}\end{array}$ & $\begin{array}{r}5.00 \\
2.17 \mathrm{E}-06 \\
1.64 \mathrm{E}-06 \\
6.55 \mathrm{E}-12\end{array}$ & $\begin{array}{r}3.00 \\
1.30 \mathrm{E}-06 \\
9.80 \mathrm{E}-07 \\
3.96 \mathrm{E}-12\end{array}$ & $\begin{array}{r}5.00 \\
2.17 \mathrm{E}-06 \\
1.69 \mathrm{E}-06 \\
6.59 \mathrm{E}-12\end{array}$ & $\begin{array}{r}4.33 \\
1.88 \mathrm{E}-06 \\
1.43 \mathrm{E}-06 \\
5.70 \mathrm{E}-12\end{array}$ \\
\hline $1,2,3,7,8$ PeCDF & $\begin{array}{l}\mathrm{Pg} \\
\mathrm{ug} / \mathrm{dscm} \\
\mathrm{ug} / \mathrm{dscm} @ 7 \% 02 \\
\mathrm{lbs} / \mathrm{hr}\end{array}$ & $\begin{array}{r}<\quad 2.00 \\
<\quad 8.68 \mathrm{E}-07 \\
<\quad 6.55 \mathrm{E}-07 \\
<\quad 2.62 \mathrm{E}-12\end{array}$ & $\begin{array}{rr}< & 1.00 \\
<\quad 4.35 \mathrm{E}-07 \\
<\quad 3.27 \mathrm{E}-07 \\
<\quad 1.32 \mathrm{E}-12\end{array}$ & $\begin{array}{rr}< & 1.00 \\
< & 4.34 \mathrm{E}-07 \\
< & 3.37 \mathrm{E}-07 \\
< & 1.32 \mathrm{E}-12\end{array}$ & $\begin{array}{r}<\quad 1.33 \\
<\quad 5.79 \mathrm{E}-07 \\
<\quad 4.40 \mathrm{E}-07 \\
<\quad 1.75 \mathrm{E}-12\end{array}$ \\
\hline $2,3,4,7,8 \mathrm{PeCDF}$ & $\begin{array}{l}\mathrm{PB} \\
\text { ug/dscm } \\
\text { ug/dscm@7\%02 } \\
\text { lbs/hr }\end{array}$ & $\begin{array}{r}<\quad 20.00 \\
<\quad 8.68 \mathrm{E}-06 \\
<\quad 6.55 \mathrm{E}-06 \\
<\quad 2.62 \mathrm{E}-11\end{array}$ & $\begin{array}{l}<\quad 10.00 \\
<\quad 4.35 \mathrm{E}-06 \\
<\quad 3.27 \mathrm{E}-06 \\
<\quad 1.32 \mathrm{E}-11\end{array}$ & $\begin{array}{r}15.00 \\
6.51 \mathrm{E}-06 \\
5.06 \mathrm{E}-06 \\
1.98 \mathrm{E}-11\end{array}$ & $\begin{aligned} &< 15.00 \\
&< 6.51 \mathrm{E}-06 \\
&< 4.96 \mathrm{E}-06 \\
&<\quad 1.97 \mathrm{E}-11\end{aligned}$ \\
\hline $1,2,3,4,7,8 \mathrm{HxCDF}$ & $\begin{array}{l}\mathrm{Pg} \\
\text { ug/dscm } \\
\text { ug/dscm@7\%O2 } \\
\mathrm{lbs} / \mathrm{hr}\end{array}$ & $\begin{array}{r}4.00 \\
1.74 \mathrm{E}-06 \\
1.31 \mathrm{E}-06 \\
5.24 \mathrm{E}-12\end{array}$ & $\begin{array}{rr}< & 2.00 \\
< & 8.70 \mathrm{E}-07 \\
< & 6.53 \mathrm{E}-07 \\
< & 2.64 \mathrm{E}-12\end{array}$ & $\begin{array}{r}4.00 \\
1.74 \mathrm{E}-06 \\
1.35 \mathrm{E}-06 \\
5.27 \mathrm{E}-12\end{array}$ & $\begin{array}{rr}< & 3.33 \\
< & 1.45 \mathrm{E}-06 \\
< & 1.10 \mathrm{E}-06 \\
<\quad 4.38 \mathrm{E}-12\end{array}$ \\
\hline
\end{tabular}


TABLE 6-26: SUMMARY OF PCDF EMISSION RATES FOR MULTIPLE ORGANIC FEEDSTOCK

(Continued)

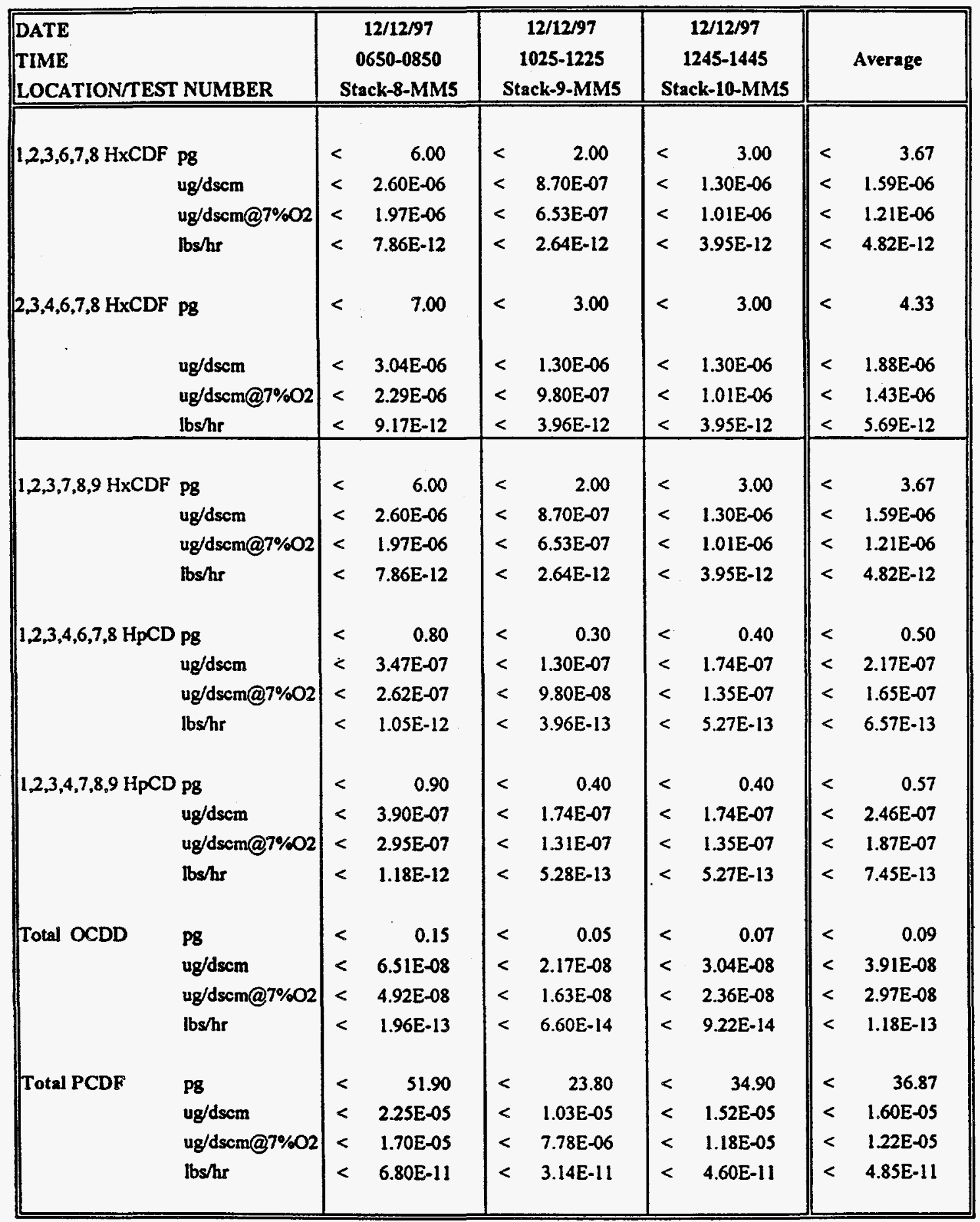

1- dsefm $=$ dry standard cubic feet per minute at $68^{\circ} \mathrm{F}$ and $29.92 \mathrm{in} . \mathrm{Hg}$ 
TABLE 6-27:

2,3,7,8-TCDD TEQ STACK EMISSIONS

\begin{tabular}{|c|c|}
\hline DESCRIPIION & ng/dscm@7\% \\
\hline Multiple Surrogate Tests: & \\
\hline - Feedstock No.6 & $<0.145$ \\
\hline - Feedstock No.2 & $<0.043$ \\
\hline Piketon Waste Tests: & \\
\hline - Test 4 & $<0.027$ \\
\hline - Test 5 & $<0.031$ \\
\hline Multiple Organic Retests: & \\
\hline - Test R3 & $<0.066$ \\
\hline
\end{tabular}




\subsubsection{Discussion of Results}

Multiple Surrogate Tests. The operating conditions summarized in Table 6-3 indicate the following:

- The surrogate waste feed rate ranged from 22.5 to $65.7 \mathrm{lb} / \mathrm{hr}$. The feed rate was the lowest with feedstock No. 4 and the highest with feedstock No. 2. The feed rate was limited by the heat input required from the electrical heaters for volatilization and the endothermic reactions. It is to be noted that the feedstock throughput is a function of waste composition, bed temperature, steam fluidization velocity, steam inlet temperature and the electrical heat input.

- The average steam flow rate into the fluidized bed ranged between 76 and $107 \mathrm{lb} / \mathrm{hr}$. The steam fluidization velocity correspondingly spanned the range of 1.17 and $1.61 \mathrm{ft} / \mathrm{s}$. The lower flow rate and velocity were satisfactory for feedstocks 2,4 and 3 but relatively higher flow rate and velocity were required for feedstocks 1 and 6 due to their sticky nature and their tendency to induce the formation of clumps in the fluidized bed.

- The dense bed average temperature remained close to $1050^{\circ} \mathrm{F}$ and the dense bed height (expanded height) averaged about $12.5 \mathrm{ft}$ in these tests. The bed temperature, however, was somewhat lower $\left(1035^{\circ} \mathrm{F}\right)$ in the case of feedstock 2 due presumably to the higher waste feed rate. Likewise, the bed height was lower $(7.5 \mathrm{ft}$ ) in Test 1 with feedstock 6 but was increased to about $12.5 \mathrm{ft}$ in the subsequent tests to improve in-bed vapor/gas residence time and to ensure bed solids coverage of the top heater tube array at all times. The electrical heat input was typically on the order of $28 \mathrm{~kW}$ except in the case of Test 1 with feedstock 6 , wherein it was approximately $20 \mathrm{~kW}$. Herein the feed rate could have been higher as additional heater capacity was available. The feed rate of feedstock 6 , however, was not increased further to avoid caking and non-uniform fluidization in the bed. 
- $\quad$ From the gas composition data, the HT HEPA2 exit gas flow rates were computed for tests with feedstocks 6, 2, 4 and 3. Gas sampling was not performed in the test with feedstock 1 and therefore none of the gas flow rates were calculated. The HT HEPA2 exit gas flow rates range between 122 and $182 \mathrm{lb} / \mathrm{hr}$ and include the fluidization steam, the volatiles and the steam reformate products generated from the organics in the waste, the nitrogen used for purging pressure taps and puffing the two HT HEPA filters, and air leakage in through the feed system and the HT HEPA filter housing. The air ingress is particularly detrimental if chlorine is present in the feedstock for it can promote the right environment (chlorine compounds in the steam reformer product gas stream, oxygen from air, particle pores as catalyst sites, and the temperature of between 400 and $800^{\circ} \mathrm{F}$ in the HT HEPA filter train) for dioxin and furan formation. Double lock hopper arrangement, superior seals and nitrogen blanketing should minimize air ingress at the feed system. Robust flanges and superior gaskets in the case of the HT HEPA filters should minimize air ingress through the filter housing. In fact, some of these modifications were carried out after the test with feedstock 6 but prior to the test with feedstock 2 and additional modifications were performed prior to the Piketon waste simulation tests.

The average pressure drop across the HT HEPAl ranged between 0.7 and 6.1 inch water. The pressure drop is generally low ( $<2$ inch water) except in the case of feedstock 6 wherein the higher pressure drop (6.1 inch water) is attributed to the higher gas flow rate passing through a fixed filter area and the increased solids carryover as a consequence of higher steam fluidization velocity. The average pressure drop across HT HEPA2 varied slightly from a mean of about 7.2 inch water. It looks like the gas flow rate and the fine particulates entering the HT HEPA2 exhibited opposing trends with a change in feedstock to essentially yield similar pressure drops in all the cases. For example, with feedstock 6, the gas flow 
rate was high but the fines concentration was relatively low due to the predominantly liquid content of this feedstock (see Table 3-1).

- Based on the gas analysis data, the thermal oxidizer exit gas flow rates were computed and these range from 1,750 to $2,700 \mathrm{lb} / \mathrm{hr}$. The flue gas comprised oxygen, nitrogen, water, $\mathrm{CO}_{2}, \mathrm{HCl}$ and $\mathrm{SO}_{2}$. The flow rates are rather high due to high air dilution rates used in ensuring high DRE for RCRA organics and Principal Organic Hazardous Constituents (POHC).

- The stack flue gas flow rate was measured with pitot tubes downstream of the vacuum blower in the case of Test 1 with feedstock 6 and registered about 12,000 $\mathrm{lb} / \mathrm{hr}$. With other feedstocks, the measurements were made upstream of the vacuum blower and tempering air inlet but downstream of the activated carbon filter. Here, the flue gas flow rates ranged between 6,800 and $8,600 \mathrm{lb} / \mathrm{hr}$. Note the significant augmentation of the flow on account of the tempering employed to regulate the fluidized bed freeboard pressure and the vacuum blower exit temperature. This augmentation or dilution impaired the accuracy of the trace emissions measurement and therefore the gas sampling location was moved upstream after Test 1 . Also, the flue gas flow rate increases approximately by a factor of 3 between the oxidizer and the carbon filter exit. A part of the increase is attributed to water vapor addition due to gas quenching in the spray cooler and subsequent wet scrubbing. But again the predominant contributor is air leakage in through flanges and the housing of the gas cleanup train components. These components were leak checked and remedial measures were taken before commencing the Piketon waste simulation tests.

The destruction and removal efficiency (DRE) values for tests with feedstocks 6, 2, 4 and 3 are furnished in Table 6-6. Gas sampling and analysis were not performed in the case of the test with feedstock 1 and therefore no DRE values are included. DRE for RCRA organics (naphthalene and 1,2-dichlorobenzene) and several POHC (phenol, toluene, tetrachloroethylene and 
ethylene glycol) are listed in the Table. The overall DRE for the system as well as the DRE for the steam reformer and that for the thermal oxidizer are given. The steam reformer DRE varies from about $33 \%$ to $99.5 \%$ depending on the organic component, waste type/matrix, the component concentration, and the dense bed average temperature. In terms of relative ease of destruction, the organic components can be ranked from high (easier) to low (more difficult) as follows: phenol, tetrachloroethylene, toluene, ethylene glycol, 1,2-dichlorobenzene, and naphthalene. Likewise, the ranking from high to low for different waste types/matrices turns out to be feedstock 6 , feedstock 4 , feedstock 3 and feedstock 2 . Incidentally, the RCRA organics concentration increases from low to high in the same order viz. feedstocks $6,4,3$ and 2. Feedstock 6 is predominantly a liquid (water, more specifically), feedstock 4 is a sludge with many types of solids, feedstock 3 is a liquid-solid mixture with vermiculite and perlite, and feedstock 2 is more of a liquid and included vermiculite. The dense bed average temperature was the highest $\left(1050^{\circ} \mathrm{F}\right)$ in the case of feedstock 6 and the lowest $\left(1035^{\circ} \mathrm{F}\right)$ in the case of feedstock 2 . It is plausible that the waste type/matrix through its heterogeneity and the organic component concentration affected the mixing, feed uniformity, and the residence time and these along with the bed temperature influenced the DRE in the steam reformer.

The overall DRE for the system exceeds $99.99 \%$ in many cases and is greater than $99 \%$ in all the cases. The DRE for the thermal oxidizer was computed from those for the steam reformer and the overall system and are included in Table 6-6. The DRE values range from about $75 \%$ to 99.99\%. Surprisingly, the oxidizer DRE values are higher for naphthalene and 1,2-dichlorobenzene than those for tetrachloroethylene and toluene. This apparent anomaly may be attributed to several factors: (i) the tetrachloroethylene and toluene stack emissions were not blank corrected using data from ambient measurements, (ii) the HT HEPA exit and stack gas measurements were not taken simultaneously but at different times, and (iii) the organic component feed could have been non-uniform/unsteady due to the heterogeneous nature of the surrogate waste. Nevertheless, the thermal oxidizer DRE values were either below or significantly lower than those expected ( $>99.99 \%$ ) based on the previous history of the Thermatrix flameless thermal oxidizer performance. During the Piketon waste simulation test program, a detailed investigation was conducted to determine the cause for this inferior performance. The problem was traced to a 
surreptitious air leak through an inadvertently open pipe coupling at the bottom of the oxidizer inlet plenum and another air leak through a disconnected pressure sensing line. These openings were then closed and the oxidizer operating temperature was increased. The subsequent tests demonstrated overall DRE on the order of $99.9999 \%$ for monochlorobenzene, a PCB surrogate. Therefore, overall DRE exceeding $99.99 \%$ is considered a certainty; in fact, additional tests with the organics listed in Table 6-6 were performed to unequivocally demonstrate this level of performance. The retest data are furnished in Table 6-24.

The surrogate radionuclides and metals retention results are presented in Table 6-7. Values are reported for the overall system as well as for just the steam reformer. The radionuclides, cerium and cesium, are almost all retained within the system due presumably to the lowtemperature operation $\left(\sim 1050^{\circ} \mathrm{F}\right)$ of the steam reformer and HT HEPA filtration. The consistently lower overall retention of cerium as compared to the cerium retention in the steam reformer seems anomalous and cannot be explained. Perhaps the saddles used in the thermal oxidizer as packing were contaminated with cerium and gave rise to trace emissions! The cesium concentration in the stack gas was consistently below the analyzer detection limit and therefore, cesium can be considered non-detect (n.d.) with regard to stack emissions. This is good news indeed considering the fact that cesium and its compounds are much more volatile (or have lower melting and boiling points) than cerium and its compounds. Therefore, cerium ought to have been also non-detect at the stack. This indeed was corroborated by the subsequent Piketon waste simulation tests wherein the cerium concentration in the stack flue gas was below the instrument detection limit. The retention of other metals (chromium, nickel, lead and cadmium) in the reformer is generally high due presumably to the low temperature $\left(\sim 1050^{\circ} \mathrm{F}\right)$ of the steam reformer and HT HEPA filtration. Chromium and nickel retention in tests with feedstocks 4 and 2 were low. This may be due to either non-uniform/unsteady metals (chromium and nickel) feed resulting from the heterogeneous nature of the waste or to metals (chromium and nickel) loss from the stainless steel components (bed vessel, cyclone, piping, HT HEPA filter housing, etc.) of the steam reformer. There seems to be some additional capture of chromium, nickel, lead and cadmium in the gas cleanup train downstream of the oxidizer and therefore, the overall metals retention is generally higher than the retention in the reformer. Two of the overall retention 
values for lead are slightly lower than the reformer retention values and are contrary to expectations. This may again be attributed to non-uniform/unsteady lead feed and nonsimultaneous gas sampling at HT HEPA exit and stack.

Table 6-8 provides the conversion, capture and closure results. The carbon conversion in the steam reformer ranged from 71 to 89 percent and this result is better than expected (60-70\%) based on past steam-reforming experience with different feedstocks at this low temperature $\left(\sim 1050^{\circ} \mathrm{F}\right)$. This favorable outcome is attributed to the high organic liquid content of the feedstocks. Based on the gas analysis data, the mass balances for the steam reformer (fluidized bed, HT HEPA1 and HT HEPA2) were computed for the first four tests. The closure is generally within 6 percent and that is very good indeed! The ash balances were computed for the four tests and the closure is nominally in the \pm 3 percent range. This again is excellent. The heat balances were performed around the steam reformer vessel including the internal cyclone. Considering the small size of the unit and the relatively large surface-area-to-volume ratio of the fluid-bed vessel, the closure within about 10 percent is quite satisfactory.

The waste volume reduction ratio for the steam reformer ranges from a low of 1.6 to a high of 8 . The mass reduction for the steam reformer spans the range between 38 and 87 percent. The largest values are obtained for feedstock 6 (natural aqueous waste) with a significant liquid content. The lowest volume reduction occurs in the case of feedstock 4 (high organic content sludge) while the lowest mass reduction occurs in the case of feedstock 1 (heterogeneous debris). Both of these feedstocks contain significant quantities of inorganic solids with feedstock 1 comprising a larger proportion on a mass basis (see Tables 3-1 and 3-3).

The $\mathrm{HCl}$ and the sulfur capture efficiency both exceed 99.5 percent for the overall system due to the efficient gas cleanup train with a spray dryer and a polishing wet scrubber. This combination with zero liquid discharge seems to be an effective means of minimizing acid gas emissions. Due to the use of caustic as a sorbent and the collection of spent salts in the baghouse, the overall volume and mass reductions are slightly lower than those for the steam reformer. 
The high-temperature HEPA2 exit gas composition data are given in Table 6-9. For the test with feedstock $6, \mathrm{H}_{2}$ and $\mathrm{CO}$ measurements were not made and therefore, the relative proportions of the fixed gases $\left(\mathrm{CO}_{2}, \mathrm{O}_{2}, \mathrm{~N}_{2}\right)$ could be somewhat erroneous: The VOC and SVOC in the gas stream are at ppmv levels; so also are $\mathrm{HCl}, \mathrm{H}_{2} \mathrm{~S}$, chlorine and ammonia. The water vapor concentration is high as. expected due to steam fluidization. The nitrogen concentration is also significant due to nitrogen purges and air leakage through the feed system and the HT HEPA filter housing. Feedstock 2 constitutes the highest organic content feed and therefore, the VOC and SVOC concentrations are the highest for that case. Likewise, $\mathrm{HCl}$ concentration for that stream is the highest due to the chlorinated organic content. The surrogate radionuclides repeat at ppbw level in the product gas. Likewise, the metals (chromium, nickel, lead and cadmium) concentration is generally in the ppbw level.

Table 6-10 shows the stack flue gas composition. The oxygen concentration is rather high due to the high excess air operation of the oxidizer and the significant air leakage in through the gas cleanup train flanges and housing. The acid gases $\left(\mathrm{HCl}\right.$ and $\left.\mathrm{SO}_{2}\right)$ report at ppbv levels attesting to the effectiveness of the gas cleanup train. Trace concentrations of VOC, SVOC (at ppbv levels) and ethylene glycol are also observed in the off-gas. As pointed out earlier, this inferior performance of the oxidizer has been traced to a surreptitious air leak into the inlet plenum of the thermal oxidizer. This problem since has been fixed and the follow-up results for destruction and removal efficiency have been excellent ( $\sim 99.9999 \%$ for PCB surrogate). Therefore, VOC and SVOC in the off-gas should drop to pptv level. Likewise, furan and dioxin emissions should be negligible both due to the minimization of air leak into the steam reformer subsystem and better performance of the oxidizer.

The PCDD and PCDF emissions data (see Tables 6-11 through 6-14) indicate trace concentrations of these species in both the steam reformer exit stream and the stack gas. Prior steamreforming tests with other feedstocks have indicated the absence of dioxin/furan formation and in fact very good destruction of dioxins and furans. Therefore, the present result was initially somewhat of a surprise. But upon examination of the test conditions, it became clear that the probable cause was air leakage into the steam reformer through the solids feed system and the 
HT HEPA filter housings. Even though Test 1 with feedstock 6 involved liquid feed, the solids feed system was on-line to feed sand and maintain bed level. The freeboard of the steam reformer was operated at negative pressure in an effort to prevent outflow. This, however, encouraged air leakage into the system through the rotary valve and injection screw seals of the solids feed system. The air ingress provided the right environment (chlorine compounds in the feedstock, oxygen from air, particle surfaces as catalyst sites, and temperature of between 400 and $800^{\circ} \mathrm{F}$ in the HT HEPA filter train) for dioxin and furan formation. This pointed out the need for leakproof seals in the feed system, nitrogen back purging, superior flanges, gaskets and tighter seals for the HT HEPA filters. These measures were implemented prior to the Piketon waste simulation tests. As shown in Tables 6-16 through 6-19, the dioxins and furans were essentially non-detectable at the parts per trillion level during the Piketon tests, confirming that the previous levels were due to air leakage at the HEPA. Because of the reducing environment of the steam reformer, dioxins and furans are actually destroyed and they would not be expected to appear anywhere in the intermediate streams. Also, as shown, the dioxin and furan levels at the stack are essentially nondetectable and well below acceptable standards.

The operating parameter trends shown in Figures 6-2 through 6-6 correspond to feedstock 2, Test 2. The trends for the other feedstocks (Nos. 6, 4,3 and 1) are included in Appendix D. In general, each of the operating parameters exhibits a trend that does not change much from one feedstock to another. Therefore, although the ensuing discussion refers to the trends for one feedstock, it is equally applicable for the other feedstock trends. Figures 6-2 through 6-6 indicate the following:

- The steam reformer fluidization flow rate of steam has been quite steady, thereby attesting to the proper operation of the steam control valve and the steam supply system.

- The steam reformer freeboard pressure was reasonably stable for the first four tests but registered some transients. The fluctuations are attributed to swings in product gas flow rate (due to fluctuations in steam fluidization flow, waste feed rate and waste composition) and dust cake concentration on the HT HEPA filters. 
- The HT HEPA1 and HT HEPA2 pressure drops in general exhibit overall stability with the progress in the test run notwithstanding interim fluctuations. The pressure drop swings are attributed to the dust cake buildup and $\mathrm{N}_{2}$ purge/ backflush cycles. Typically, HT HEPAl registers a higher pressure drop than HT HEPA2 due probably to the higher solids loading and the higher gas temperature; the higher solids loading tends to constrict gas flow area while the higher gas temperature tends to increase the flow velocity. Both measures lead to increased pressure drop.

- $\quad$ All the temperatures (bed average, cyclone exit, HT HEPA2 exit and oxidizer average) are quite steady during the test. The stable steam reformer temperatures are attributed to fluid-bed capacitance and satisfactory performance of the bed electrical heater controls. The stable oxidizer temperatures stem from reliable operation of the oxidizer PLC.

- The electrical input to the fluid bed is also stable during the test run. There are a few small fluctuations in response to feed swings, freeboard pressure variation and changes in steam fluidization flow. This indicates that the electrical heater controls not only performed admirably, but also exhibited fast response.

Piketon Waste Simulation Tests. The operating conditions summarized in Table 6-4 indicate the following:

- The waste feed rate ranged from 11 to $17.3 \mathrm{lb} / \mathrm{hr}$. The feed rate had to be cut back with an increase in organic (monochlorobenzene or ethylene glycol) content. The thermal oxidizer was rated for a nominal throughput of $400 \mathrm{scfm}$ of product gasair mixture and this essentially limited the total waste feed rate into the steam reformer. In this instance, the electrical heaters in the steam reformer did not limit the feed rate. 
- The steam flow rate into the fluidized bed averaged about $103 \mathrm{lb} / \mathrm{hr}$. The steam fluidization velocity was on the order of $1.8 \mathrm{ft} / \mathrm{s}$. A higher fluidization velocity was required here than those in the multiple surrogate tests due to the high specific volume and cohesivity of the Piketon waste.

- The dense bed temperature averaged about $1200^{\circ} \mathrm{F}$ and the dense bed height (expanded height) was on the order of $5.8 \mathrm{ft}$. A higher bed temperature was employed here as compared to those in the multiple surrogate tests because of the thermal stability of the radionuclide (cerium compound here but this holds for uranium compound as well which is the radionuclide in the actual Piketon waste) and the desire to maximize carbon conversion and in turn maximize volume reduction. The electrical heat input ranged from 19.4 to $24.6 \mathrm{~kW}$ and as stated earlier surplus heat capacity was available to treat more waste if the oxidizer had not been limiting.

- From the gas composition data, the HT HEPA2 exit gas flow rates were computed. The HT HEPA2 exit gas flow rates range between 170 and $198 \mathrm{lb} / \mathrm{hr}$ and include the fluidization steam, the volatiles and the steam reformate products generated from the organics in the waste, the nitrogen used for purging pressure taps and puffing the two HT HEPA filters, and air leakage in through the feed system and the HT HEPA filter housing. The air ingress is particularly detrimental if chlorine is present in the feedstock for it can promote the right environment (chlorine compounds in the steam reformer product gas stream, oxygen from air, particle pores as catalyst sites, and the temperature of between 400 and $800^{\circ} \mathrm{F}$ in the HT HEPA filter train) for dioxin and furan formation. Attempts were made prior to the start of this test campaign to minimize air ingress into the system.

- The average pressure drop across the HT HEPAl ranged between 1.2 and 8.4 inch water. The pressure drop tends to increase with an increase in total feed rate or conversely decrease with an increase in organic content. The average pressure 
drop across HT HEPA2 ranged from 1.0 to 3.1 inch water. It looks like the solids loading into the HT HEPA2 is relatively low and therefore the pressure drop is generally small.

- Based on the gas analysis data, the thermal oxidizer exit gas flow rates were computed and these range from 1,890 to $2,230 \mathrm{lb} / \mathrm{hr}$. The flue gas comprised oxygen, nitrogen, water, $\mathrm{CO}_{2}, \mathrm{HCl}$ and $\mathrm{SO}_{2}$. The flow rates are rather high due to high air dilution rates used in ensuring high DRE for RCRA organics and Principal Organic Hazardous Constituents (POHC).

- The stack flue gas flow rate was measured with pitot tubes upstream of the vacuum blower and tempering air inlet but downstream of the activated carbon filter. The flue gas flow rates measured were on the order of $3,000 \mathrm{lb} / \mathrm{hr}$. The flue gas flow rate increases somewhat between the oxidizer and the carbon filter exit. This increase is mostly attributed to water vapor addition due to gas quenching in the spray cooler and subsequent wet scrubbing. There is probably a slight contribution from air leakage in through flanges and the housing of the gas cleanup train components. These components were leak checked and remedial measures were taken before commencing the Piketon waste simulation tests. Consequently, the stack gas flow rates have decreased significantly from the levels in the case of multiple surrogate tests (see Table 6-3).

The destruction and removal efficiency (DRE) values are furnished in Table 6-15. DRE for monochlorobenzene, a PCB surrogate and ethylene glycol are listed in the Table. The overall DRE for the system as well as the DRE for the steam reformer and that for the thermal oxidizer are given. The steam reformer DRE varies from about $91 \%$ to $99+\%$ depending on the organic component and the component concentration. It seems that both monochlorobenzene and ethylene glycol offer similar levels of resistance to destruction. The DRE variation with monochlorobenzene concentration is non-linear and mixed. The DRE is low at low concentration 
(Tests 1 and 2), high at medium concentration (Test 5), and medium at high concentration. The mixed trend may be due to feed non-uniformity.

The overall DRE for the system registers $99.9999 \%$ after the problem with the thermal oxidizer was corrected. The ethylene glycol concentration in both the HT HEPA2 exit and stack gas samples were below detection limit and therefore the DRE values for Test 6 correspond to lower bounds. The DRE for the thermal oxidizer was computed from those for the steam reformer and the overall system and are included in Table 6-15. The DRE values are on the order of $99.99 \%$. For Tests 1 and 2 , the thermal oxidizer DRE values were lower than those expected ( $>99.99 \%$ ) based on the previous history of the Thermatrix flameless thermal oxidizer performance. Therefore, prior to Test 3, a detailed investigation was conducted to determine the cause for this inferior performance. The problem was traced to a surreptitious air leak through an inadvertently open pipe coupling at the bottom of the oxidizer inlet plenum and another air leak through a disconnected pressure sensing line. These openings were then closed and the oxidizer operating temperature was increased. The subsequent tests demonstrated overall DRE on the order of $99.9999 \%$ for monochlorobenzene, a PCB surrogate. Similarly, overall DRE exceeding $99.99 \%$ is considered a certainty for ethylene glycol; in fact, additional tests with ethylene glycol and other organics were performed to unequivocally demonstrate this level of performance. The retest data are furnished in Table 6-24.

The surrogate radionuclide retention results are presented in Table 6-16. Values are reported for the overall system as well as for just the steam reformer. The radionuclide cerium is all retained within the system due presumably to the low-temperature operation $\left(\sim 1200^{\circ} \mathrm{F}\right)$ of the steam reformer and HEPA filtration. The cerium concentration in the stack gas was consistently below the analyzer detection limit and therefore, cerium can be considered non-detect (n.d.) with regard to stack emissions.

Table 6-17 provides the conversion, capture and closure results. The carbon conversion in the steam reformer ranged from 98 to $99+$ percent and this result is very encouraging. This favorable outcome is attributed to the modest bed temperature $\left(\sim 1200^{\circ} \mathrm{F}\right)$ and the high organic 
carbon content of the feedstock. Based on the gas analysis data, the mass balances for the steam reformer (fluidized bed, HT HEPA1 and HT HEPA2) were computed for five of the six tests. As discussed previously, Test 3 was a screening test and detailed measurements were not taken to perform closure. The closure is generally within 10 percent and that is very good indeed! The ash balances were computed for the four tests and the closure is nominally in the \pm 1 percent range. This again is excellent. The heat balances were performed around the steam reformer vessel including the internal cyclone. Considering the small size of the unit and the relatively large surface-area-to-volume ratio of the fluid-bed vessel, the closure within about 15 percent is quite satisfactory.

The waste volume reduction ratio for the steam reformer ranges from a low of 913 to a high of 2,675 . The mass reduction for the steam reformer spans the range between 95.3 and 98.4 percent. It is understood that the highest concentration of Uranium in the polypropylene pads at Portsmouth is approximately $.022 \%$, thus the nominal concentration in the ThermoChem tests was $.03 \%$. Some of the tests were run at higher Cerium concentrations in the surrogate feed material, however. The highest concentration of Cerium experienced in the steam reformer reactor bed material was $.028 \%$ after the 750 -hour test run. Higher Cerium concentrations were found in the HEPA catches and the mass balance shows most of the Cerium reported to the HEPA catches. The average Cerium concentration in the mixed total HEPA catch was $0.03 \%$, while the highest measured Cerium concentration in the HEPA catch was $1.7 \%$. At present it is not known whether this concentration level can lead to criticality. Experts in nuclear reactor dynamics will be consulted and modeling calculations will be performed during the detailed process design stage to design a safe, non-critical system. It is anticipated that the detailed design will include a radiation monitoring system and that the HEPA catch will be continuously removed to a non-critical geometric assembly unit. A similar system will be installed for the reactor bed material, if required.

The $\mathrm{HCl}$ capture efficiency exceeds 99.5 percent for the overall system due to the efficient gas cleanup train with a spray dryer and a polishing wet scrubber. This combination with zero liquid discharge seems to be an effective means of minimizing acid gas emissions. 
The high-temperature HEPA2 exit gas composition data are given in Table 6-18. The VOC and SVOC in the gas stream are at ppmv levels; so also are $\mathrm{HCl}$, and chlorine. The water vapor concentration is high as expected due to steam fluidization. The nitrogen concentration is also significant due to nitrogen purges and air leakage through the feed system and the HT HEPA filter housing. The surrogate radionuclide reports a ppbw level in the product gas.

Table 6-19 shows the stack flue gas composition. The oxygen concentration is moderate due to the high excess air operation of the oxidizer. Tests 4,5 and 6 operate a lower $\mathrm{O}_{2}$ concentration than those in Tests 1 and 2 due to the shutting-off of the air leak into the thermal oxidizer. The acid gases $\left(\mathrm{HCl}\right.$ and $\left.\mathrm{SO}_{2}\right)$ report at ppbv levels attesting to the effectiveness of the gas cleanup train. Trace concentrations of VOC, SVOC (at ppbv levels) and ethylene glycol are also observed in the off-gas. Likewise, furan and dioxin emissions are negligible both due to the minimization of air leak into the steam reformer subsystem and better performance of the oxidizer.

The PCDD and PCDF emissions data (see Tables 6-20 through 6-23) indicate trace concentrations of these species in the steam reformer exit stream and negligible concentration in the stack gas. Prior steam-reforming tests with other feedstocks have indicated the absence of dioxin/furan formation and in fact very good destruction of dioxins and furans. It seems that a slight air leak still persists despite remedial measures to prevent air ingress. This air ingress provided the right environment (chlorine compounds in the feedstock, oxygen from air, particle surfaces as catalyst sites, and temperature of between 400 and $800^{\circ} \mathrm{F}$ in the HT HEPA filter train) for dioxin and furan formation. The thermal oxidizer, however, is efficient in destroying dioxins and furans and consequently, the dioxin and furan stack emissions are negligible.

The operating parameter trends shown in Figures 6-7 through 6-11 correspond to Test 1. The trends for the other tests (Nos. 2, 3, 4, 5 and 6) are included in Appendix D. In general, each of the operating parameters exhibits a trend that does not change much from one test to another. Therefore, although the ensuing discussion refers to the trends for one test, it is equally applicable for the other test trends. Figures 6-7 through 6-11 indicate the following: 
- The steam reformer fluidization flow rate of steam has been quite steady, thereby attesting to the proper operation of the steam control valve and the steam supply system.

- The steam reformer freeboard pressure was reasonably stable but registered some transients. The fluctuations are attributed to swings in product gas flow rate (due to fluctuations in steam fluidization flow, waste feed rate and waste composition) and dust cake concentration on the HT HEPA filters.

- The HT HEPA1 and HT HEPA2 pressure drops in general exhibit overall stability with the progress in the test run notwithstanding interim fluctuations. The pressure drop swings are attributed to the dust cake buildup and $\mathrm{N}_{2}$ purge/ backflush cycles. Typically, HT HEPAl registers a higher pressure drop than HT HEPA2 due probably to the higher solids loading and the higher gas temperature; the higher solids loading tends to constrict gas flow area while the higher gas temperature tends to increase the flow velocity. Both measures lead to increased pressure drop.

- All the temperatures (bed average, cyclone exit, HT HEPA2 exit and oxidizer average) are quite steady during the test. The stable steam reformer temperatures are attributed to fluid-bed capacitance and satisfactory performance of the bed electrical heater controls. The stable oxidizer temperatures stem from reliable operation of the oxidizer PLC.

- The electrical input to the fluid bed is also stable during the test run. There are a few small fluctuations in response to feed swings, freeboard pressure variation and changes in steam fluidization flow. This indicates that the electrical heater controls not only performed admirably, but also exhibited fast response. 
Multiple Organic Feedstock Retests. The operating conditions summarized in Table 6-5 indicate the following:

- The total feed rate ranged from 18 to $58 \mathrm{lb} / \mathrm{hr}$. The feed rate was the highest with the ethylene glycol/vermiculite mixture. Actually, an even higher feed rate was desired in view of the low sensitivity/high minimum detection limit for ethylene glycol analysis. But the feed system limited the feed rate due to the relatively low bulk density of this mixture. The feed rates of the other two organics (Tests R2 and R3) were sufficient to determine DRE at $99.9999 \%$ level.

- The steam flow rate into the fluidized bed averaged about $91 \mathrm{lb} / \mathrm{hr}$. The steam fluidization velocity was on the order of $1.4 \mathrm{ft} / \mathrm{s}$.

- The dense bed temperature averaged about $1,050^{\circ} \mathrm{F}$ and the dense bed height (expanded height) was on the order of $13.4 \mathrm{ft}$. These conditions are similar to those in the multiple surrogate tests (see Table 6-3). The electrical heat input ranged from 33 to $39.9 \mathrm{~kW}$. The heaters were operating in the 70 to $85 \%$ capacity range.

- The average pressure drop across the HT HEPA1 ranged between 2.8 and 14.4 inch water and that across HT HEPA2 spanned the range from 2.9 to 6.1 inch water. Both filters registered the highest pressure drop in Test R2 with the naphthalene/oil/sorbent pad feed. It seems that the differential pressure sensing lines on the two HEPA filters were plugged during Test R2 and therefore were reading high. For some reason, the nitrogen purge flows had dropped some time during the transition from Test R1 to Test R2 and caused the problem. After Test R2, the differential pressure sensing lines were cleared and the pressure drops returned to normal. Therefore, it seems that HT HEPAl pressure drop should actually have ranged between 3 and 6 inch water and the HT HEPA2 pressure drop should actually have averaged about 3 inch water. 
- Based on the gas analysis data, the thermal oxidizer exit gas flow rates were computed and these range from 1,780 to $1,794 \mathrm{lb} / \mathrm{hr}$. The flue gas comprised oxygen, nitrogen, water, and $\mathrm{CO}_{2}$. The flow rates are rather high due to high air dilution rates used in ensuring high DRE for RCRA organics and Principal Organic Hazardous Constituents (POHC).

- The stack flue gas, flow rate was measured with pitot tubes upstream of the vacuum blower and tempering air inlet but downstream of the activated carbon filter. The flue gas flow rates measured were on the order of $2,500 \mathrm{lb} / \mathrm{hr}$. The flue gas flow rate increases somewhat between the oxidizer and the carbon filter exit. This increase is mostly attributed to water vapor addition due to gas quenching in the spray cooler and subsequent wet scrubbing. There is probably a slight contribution from air leakage in through flanges and the housing of the gas cleanup train components.

The overall destruction and removal efficiency (DRE) values are furnished in Table 6-24. DRE for ethylene glycol, naphthalene, toluene, 1,2-dichlorobenzene and tetrachloroethylene are listed in the Table. The overall DRE for the system exceeds $99.99 \%$ in all cases and registers $99.9999 \%$ with naphthalene and 1,2-dichlorobenzene. The ethylene glycol concentration in the stack gas samples were below detection limit and therefore the DRE values for Test R1 correspond to lower bounds. Toluene detection limit was higher than normal due to contamination from toluene used as the SW-846 Method 0023 sampling train recovery solvent in measuring dioxins and furans. As a result, the test results were only sufficient to demonstrate $99.99 \%$ DRE compared to at least $99.999 \%$ for the other two compounds viz. 1,2dichlorobenzene and tetrachloroethylene.

The PCDD and PCDF emissions data (see Tables 6-25 and 6-26) indicate negligible concentration of these species in the stack gas. 
Table 6-27 shows the dioxin and furan emissions on a 2,3,7,8-TCDD toxic equivalent basis. It turns out that the stack emissions in all the cases are order of magnitude lower than the 40 CFR 60 New Source Performance Standards (NSPS) for municipal solid waste (MSW) combustors. The measurements for feedstock 6 were made downstream of the vacuum blower and therefore included a significant quantity of ambient air drawn in for tempering. The emissions were not corrected for the ambient concentration and consequently are somewhat higher. The measurements for feedstock 2 were made downstream of the carbon filter/upstream of the vacuum blower and tempering air inlet. There may still be some influence of ambient concentration due to air leakage in through gas cleanup train components. It is also to be noted that both these measurements were made prior to the discovery and correction of the problem with the thermal oxidizer. The most relevant data here correspond to Tests 4 and 5 with the Piketon waste and Test R3 with multiple organics. This is because these tests were performed after fixing the oxidizer problem and also after minimizing the air ingress. The stack emissions are all less than $0.066 \mathrm{ng} / \mathrm{dscm} @ 7 \% \mathrm{O}_{2}$ of 2,3,7,8-TCDD TEQ and this is remarkable since these are even lower than the most stringent standard viz. the European Economic Community (EEC) limit of $0.1 \mathrm{ng} / \mathrm{Nm}^{3}$.

From the solids samples collected and analyzed, the cerium loading and partitioning was tracked. Figure 6-12 shows the cerium buildup with elapsed time. Elapsed time here corresponds to the run time from the start of the test program, i.e., the first time feed of the ceriumdoped waste. There is a gradual buildup of cerium in the bed. However, the cerium preferentially reports to the HT HEPA catch rather than the bed as seen from the significantly higher concentration of HEPA catch as compared to the bed cerium concentration. The gas analysis data indicated that almost all of the cerium is retained within the steam reformer. Therefore, a cerium balance was performed by accounting for the cerium fed and the cerium collected. A total of 1.6 pounds of cerium was fed while only about 0.4 pounds of cerium could be accounted for in all of the collected solids. This poor closure may be due to one or more of the following: 


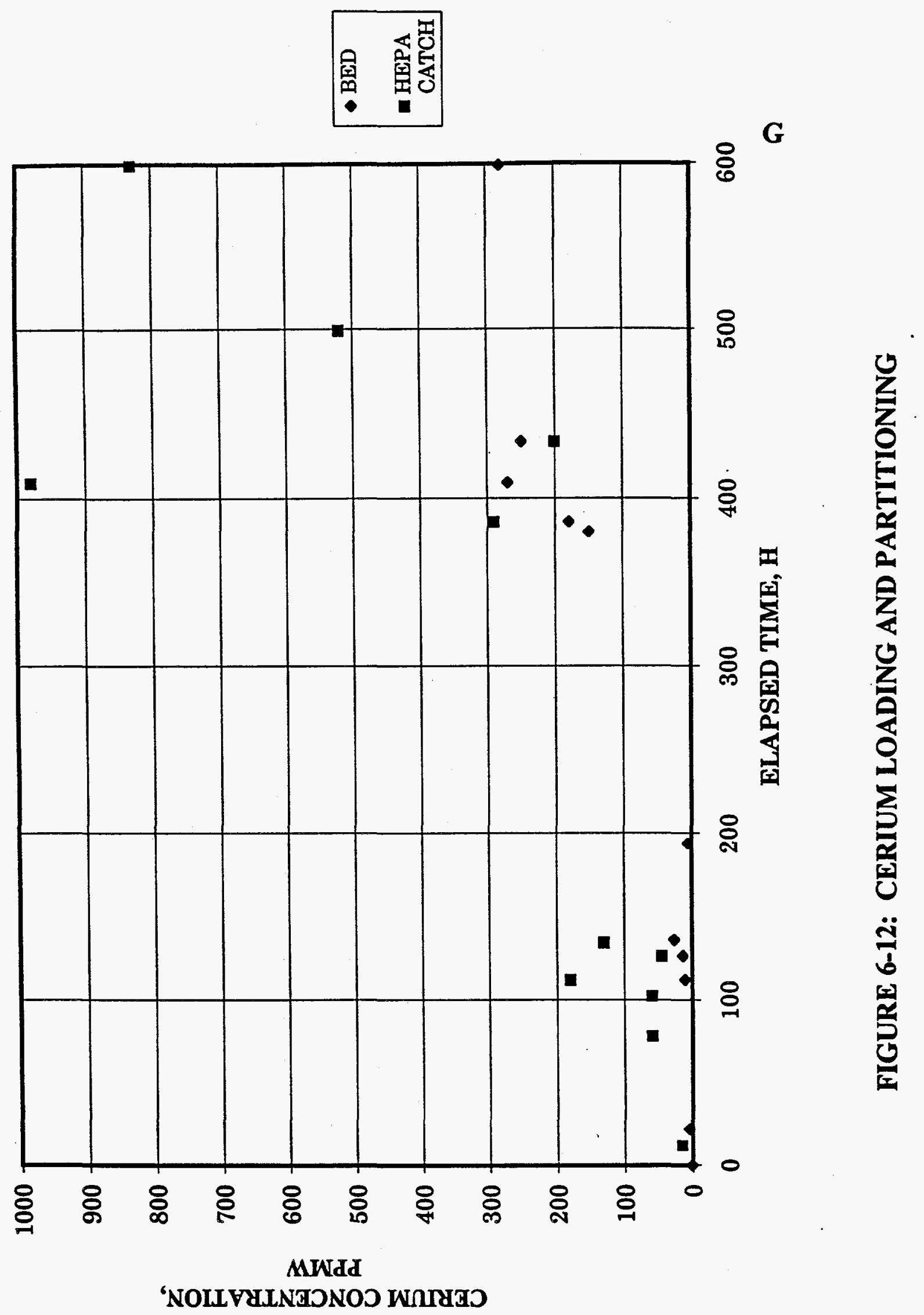


- Error in cerium analysis

- Unrepresentative solid samples

- Cerium in the form of very fine powder:

$>$ coating/depositing on pipe inner walls

$>$ caking/collecting on the ceramic membrane filter elements.

Further investigation is in progress to identify the cause for this discrepancy and achieve good balance.

\subsection{VITRIFICATION TESTS}

A study was conducted to evaluate steam reforming followed by vitrification as a means of stabilizing organic materials contaminated with radioactivity. The specific application evaluated in this case involved steam reformer fluidized bed material at the end of the feedstock No. 2 test (see Table 6-3). The products of the steam-reforming process was predominately sand and ash. A sample from the bed material was supplied to the Clemson Environmental Technologies Laboratory for subsequent vitrification processing. Included in this effort was selection of a glass composition, crucible-scale processing trials and evaluation of the glass's leaching resistance via TCLP testing.

\subsubsection{Experimental}

An analysis of the residue was performed by Centre Analytical Laboratories, Inc. and the chemical analysis is listed below in Table 6-28. 
TABLE 6-28:

\section{CHEMICAL ANALYSIS OF BED RESIDUE}

\begin{tabular}{|l|c|}
\hline \multicolumn{1}{|c|}{ Parameter } & Amount (Wt\%) \\
\hline Carbon & 0.60 \\
\hline Hydrogen & $<0.10$ \\
\hline Sulfur & $<0.10$ \\
\hline Nitrogen & $<0.10$ \\
\hline Chloride & 0.021 \\
\hline Ash & 98.52 \\
\hline
\end{tabular}

An RCRA Metals analysis was also performed by Centre Analytical Laboratories. This analysis is listed below in Table 6-29.

TABLE 6-29:

RCRA METALS ANALYSIS OF BED RESIDUE

\begin{tabular}{|l|c|}
\hline \multicolumn{1}{|c|}{ Parameter } & Amount (mg/kg) \\
\hline Chromium - total & 16.40 \\
\hline Nickel - total & 46.70 \\
\hline Lead - total & 27.70 \\
\hline Cadmium - total & 26.30 \\
\hline
\end{tabular}

A supplemental analysis was performed by CETL in the Clemson University Electron Microscope Facility using Electron Dispersion Spectroscopy (EDS). This analysis was required to better define the potential glass-forming constituents present. This analysis was performed with an accelerating voltage of $15 \mathrm{keV}$ and a takeoff angle of $30^{\circ}$. 
The results from the supplemental analysis performed using EDS are given below in Table 6-30.

TABLE 6-30:

RESULTS FROM EDS

\begin{tabular}{|l|c|}
\hline & Anount \\
\hline Carbon & 39.61 \\
\hline Oxygen & 38.49 \\
\hline Sodium & 0.73 \\
\hline Magnesium & 1.16 \\
\hline Aluminum & 0.86 \\
\hline Silicon & 17.96 \\
\hline Sulfur & 0.20 \\
\hline Potassium & 0.20 \\
\hline Iron & 0.79 \\
\hline
\end{tabular}

A series of glass compositions was developed from these analyses to be tested in crucible studies in the Deltech laboratory furnace. The developed glass compositions and their respective soak temperatures are shown in Table 6-31. 
TABLE 6-31:

GLASS COMPOSITIONS

\begin{tabular}{|c|c|c|c|c|c|c|}
\hline & Sample \#1 & Sample $\# 2$ & Sample \#3 & Sample $\# 4$ & Sample \#5 & Sample:t6 \\
\hline Compound & (\%) & (\%) & $(\%)$ & $(\%)$ & $(\%)$ & $(\%)$ \\
\hline Residue & 47.14 & 22.25 & 29.38 & 28.16 & 24.78 & 22.36 \\
\hline $\mathrm{SiO}_{2}$ & 28.22 & 53.10 & 35.96 & 34.48 & 30.33 & 27.36 \\
\hline $\mathrm{CaCO}_{3}$ & 0 & 0 & 17.70 & 12.70 & 0 & 10.08 \\
\hline $\mathrm{Na}_{2} \mathrm{CO}_{3}$ & 10.00 & 10.00 & 16.95 & 24.69 & 19.25 & 20.59 \\
\hline $\mathrm{Li}_{2} \mathrm{CO}_{3}$ & 14.64 & 14.64 & 0 & 0 & 25.63 & 19.61 \\
\hline $\begin{array}{l}\text { Soak } \\
\text { Temperature }\end{array}$ & $1150^{\circ} \mathrm{C}$ & $1150^{\circ} \mathrm{C}$ & $1200^{\circ} \mathrm{C}$ & $1200^{\circ} \mathrm{C}$ & $1200^{\circ} \mathrm{C}$ & $1200^{\circ} \mathrm{C}$ \\
\hline
\end{tabular}

All of the samples were batched to a total batch size of 50 grams. They were placed in the Deltech laboratory furnace in alumina crucibles at $850^{\circ} \mathrm{C}$ and ramped at $3^{\circ} \mathrm{C} / \mathrm{min}$ to their respective soak temperatures and allowed to soak overnight, approximately 15 hours. The samples were then removed from the furnace at their respective soak temperatures and tilted to determine if the sample was molten and, if molten, to determine if it would pour from crucible.

Once a glass composition was chosen a sample of the glass was sent to General Engineering Laboratories (GEL) for duplicate TCLP analyses and a glass composition analysis which was done on a metals basis.

\subsubsection{Results}

Samples \#5 and \#6 were the only formulations that melted. The other samples did not melt because of inadequate flux in the batch and the soak temperatures were not high enough. Sample \#6 was the most fluid and was chosen as the best composition. A sample of this glass was sent to GEL for TCLP testing and the results are below in Table 6-32. 
TABLE 6-32:

TCLP RESULTS

\begin{tabular}{|c|c|c|c|c|}
\hline Metal Analysis: & Samplet:-1. & Sample: If 2 & Units & Regulation \\
\hline Mercury & $<0.0200$ & $<0.0200$ & $\mathrm{mg} / \mathrm{l}$ & $0.20 \mathrm{mg} / \mathrm{l}$ \\
\hline Silver & $<0.010$ & $<0.010$ & $\mathrm{mg} / \mathrm{l}$ & $5.0 \mathrm{mg} / 1$ \\
\hline Arsenic & $<0.010$ & $<0.010$ & $\mathrm{mg} / \mathrm{l}$ & $5.0 \mathrm{mg} / \mathrm{l}$ \\
\hline Barium & 0.662 & 0.102 & $\mathrm{mg} / \mathrm{l}$ & $100 \mathrm{mg} / \mathrm{l}$ \\
\hline Cadmium & $<0.010$ & $<0.010$ & $\mathrm{mg} / \mathrm{l}$ & $1.0 \mathrm{mg} / \mathrm{l}$ \\
\hline Chromium & $<0.010$ & $<0.010$ & $\mathrm{mg} / \mathrm{l}$ & $5.0 \mathrm{mg} / \mathrm{l}$ \\
\hline Lead & $<0.010$ & $<0.010$ & $\mathrm{mg} / \mathrm{l}$ & $5.0 \mathrm{mg} / \mathrm{l}$ \\
\hline Selenium & $<0.010$ & $<0.010$ & $\mathrm{mg} / \mathrm{l}$ & $5.7 \mathrm{mg} / \mathrm{l}$ \\
\hline
\end{tabular}

\subsubsection{Conclusion}

Glass composition \#6 effectively stabilized any RCRA metals contained in the steam reformer beds solids residue based on the TCLP results obtained. The glass also exhibited good processability based on its fluidity at the processing temperature. 


\section{SECTION 7.0}

\section{PRELIMINARY CONCEPTUAL DESIGN}

\subsection{PROCESS FLOW OVERVIEW}

This section presents the preliminary conceptual design of a steam-reforming facility capable of processing a nominal 500 pound per hour of low-level mixed waste. This conceptual design was based on PDU performance.

The process is capable of processing a wide variety of wastes; however, it is best applied to wastes that contain a significant fraction of organic materials to maximize volume reduction. Therefore, the conceptual facility was designed to process 500 pounds per hour of spent ionexchange resin. In addition, the capability of this facility to process absorbent pads contaminated with PCB and Uranium was determined ( 300 pounds per hour). Both mass and energy balances are presented.

\subsubsection{Conceptual Facility Description}

Figures $7-1$ and $7-2$ present the block flow diagram of the conceptual facility and the mass and energy balances for the two selected waste feeds. When applied to ion-exchange resins, the facility processes 500 pounds per hour. When processing absorbent pads contaminated with PCB and Uranium, the throughput drops to 300 pounds per hour due to the higher endothermic heat required to process this waste and the increased energy content of the gas produced from this waste. In both cases, the waste mass reduction is approximately 95 percent. Each of the major sections in the conceptual facility are described below.

Waste Receiving, Preparation, and Feeding. The initial screening and characterization step would be based on review of inventory documentation for each container to identify general waste characteristics. Receipt inspection also verifies the suitability of the shipping container for further handling and staging within the process through verification of its integrity and the absence of deterioration or evidence of leaks. Radiation detection and manifest verification will be 


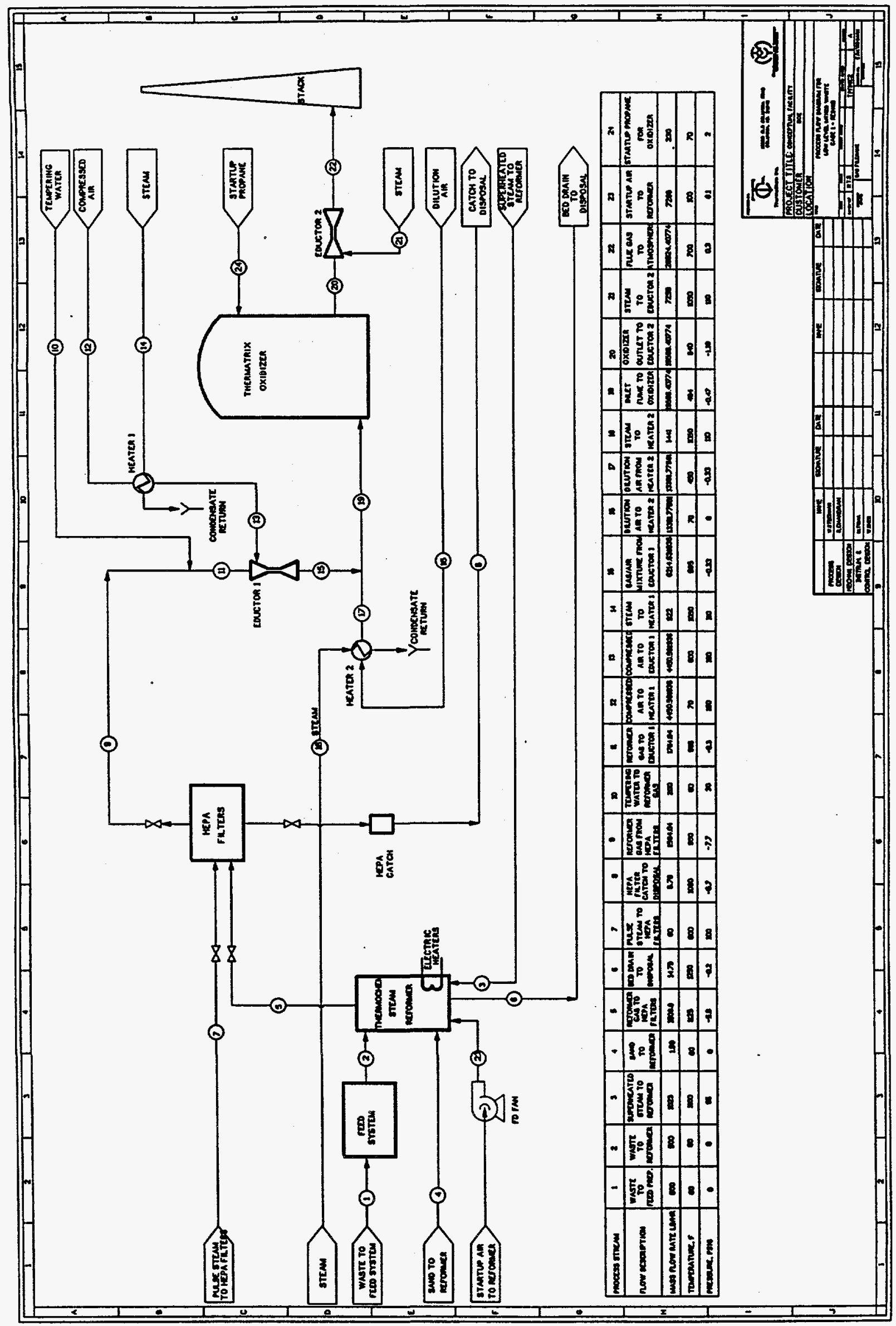

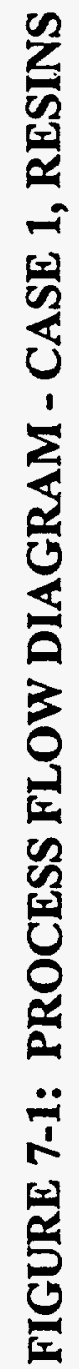




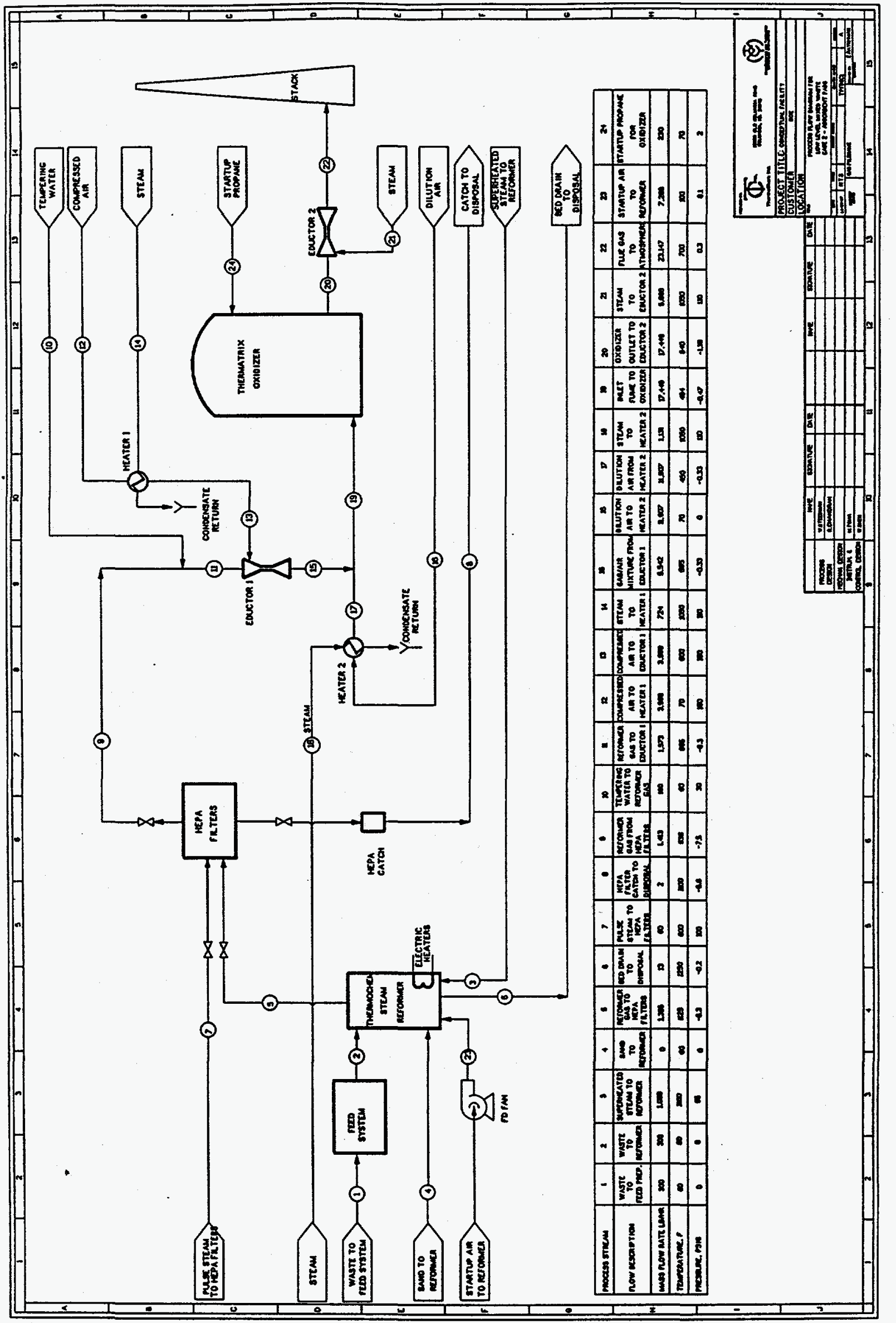

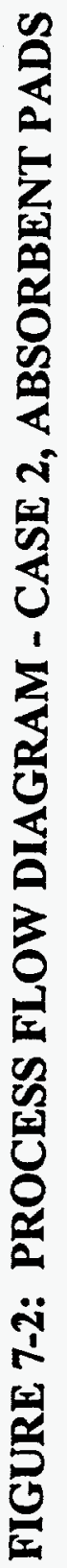


provided by hand-held detectors appropriate for the radionuclides expected, or that could be found, in the waste.

It is anticipated that initial sorting operations, if required, would be performed in a glovebox environment with containers loaded through air-lock doors with a drum handler. The glovebox would be provided with a sorting table designed to capture and collect aqueous wastes and provides lighting, air and power connections for tools. Hoisting means would provided up to and within the glove box.

Sorted, combustible waste will be reduced to uniform size (nominally less than 2 inches) if required in a two-stage chopper/shredder. Waste is fed into the shredder through an air lock to contain dust and fines. The prepared waste is discharged from the shredder into the feed hopper. The prepared waste is then fed into the steam reformer by dual water-cooled screw conveyors.

The feed hopper is a "live bottom" hopper with a volume of 400 cubic feet, approximately 7 feet square by 8 feet high. This volume is sufficient to provide 8 hours of retention time when processing absorbent pads and over 2.5 days when processing resins. Screw conveyors completely covering the bottom of the hopper discharge through a lock hopper into the injection screws which convey the feed to the steam reformer. All material-handling systems are maintained under negative pressure to maximize containment, dust and contaminant control. Fire prevention is accomplished by administrative controls (such as sorting procedures) and design features, including the exclusion of spark sources and installation of a fire suppression system.

An area radiological monitoring subsystem would be provided for safeguarding worker, public, and environmental health and safety. Equipment will be provided to monitor the treatment area and any other areas where radioactive materials could be encountered to ensure that contamination levels in these areas are kept well within established limits. Area monitors would be located at work stations and in areas where radioactive material is expected to temporarily be accumulated. Monitors would be installed at all system discharge points to continuously monitor 
the radioactivity, among other constituents, as required by regulatory commitment and a higher commitment to worker and public health and safety.

Steam Reformer. A drawing of the steam reformer is presented in Figure 7-3. The reformer is a refractory-lined vessel heated indirectly by electric resistance heaters. The heaters are capable of providing over 600 kilowatts; however, the balances are based on a heat input equivalent to 492 kilowatts.

The operating temperature of the indirectly heated fluidized bed steam reformer is between $1050^{\circ} \mathrm{F}$ and $1350^{\circ} \mathrm{F}$. The mass and energy balances presented incorporate an operating temperature of $1250^{\circ} \mathrm{F}$. The reformer operates at negative pressure to assure control of fugitive emissions that might otherwise occur through the feed system or leaking flanges.

Steam used to fluidize the reformer reacts with carbon in the waste feed to form carbon monoxide and hydrogen. The radioactive isotopes remain as part of the solids that comprise the dense fluidize bed. Other constituents of the bed solids are unreacted carbon and sand or other inert seed material. The mean particle size is approximately 250 microns. Bed solids are removed as required to maintain the appropriate operating bed height and discharged for final disposal. Options for ultimate disposal were discussed previously. Internal cyclones return most of the elutriated particles to the dense fluidized bed. Fine particles that pass through the cyclone are separated from the reformer product gas by high temperature HEPA filters.

For this preliminary conceptual design, the reformer is a scaled-up version of the PDU reformer. An alternate reformer concept that needs to be evaluated is to construct the vessel with stainless steel and insulate the outside. While the steel cost of this design will obviously be more expensive, the cost of refractory will be considerably more than that of insulation, and the reformer will be much lighter making it easier to transport. Furthermore, the potential requirement to dispose of the refractory at the end of its useful life as low-level waste will be avoided. 

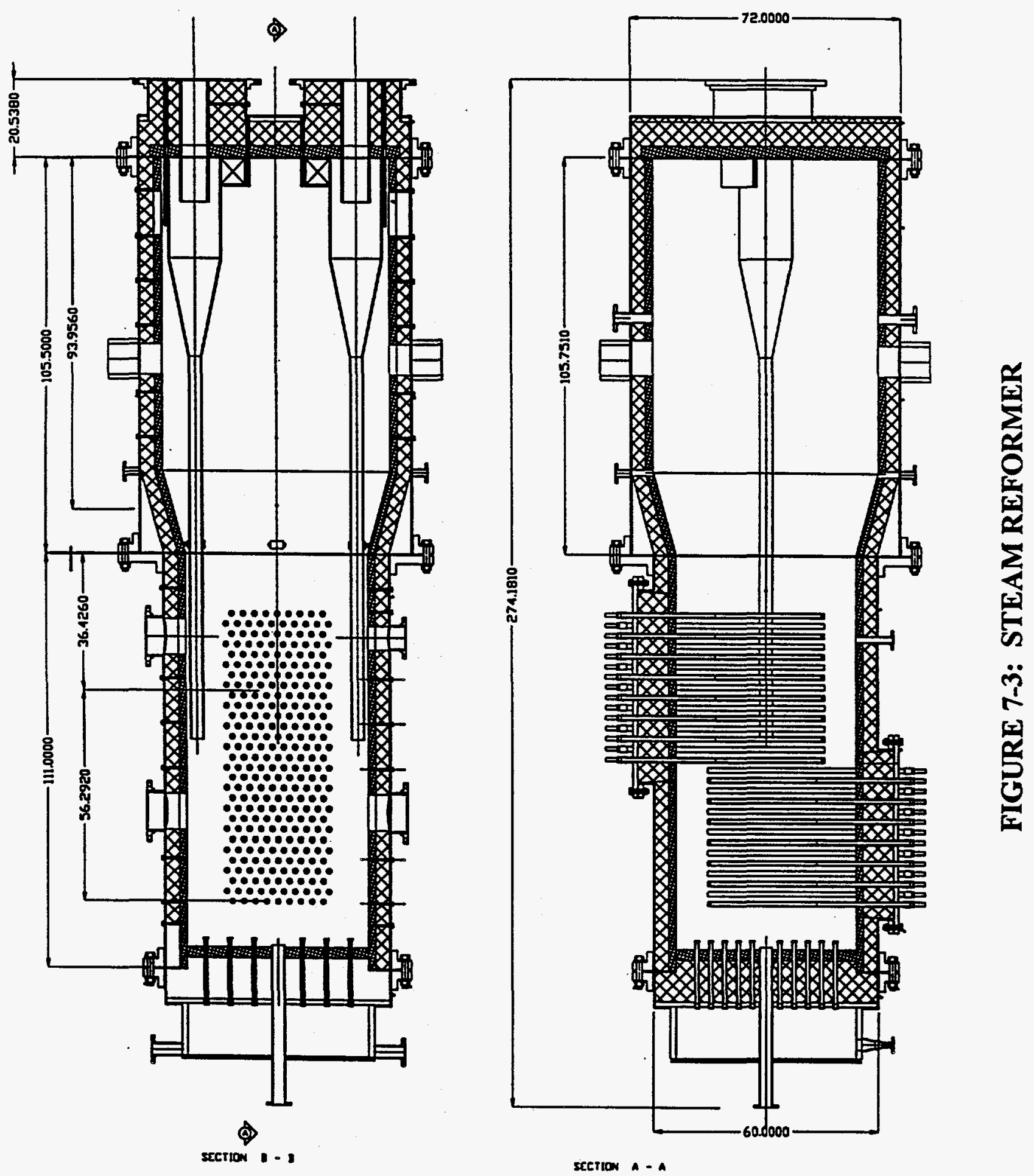
HEPA Filters. Four high temperature HEPA filters are used to assure that radioactive . constituents are retained in the reformer section of the facility. Two filters work in parallel with the second pair installed in series for redundancy.

Each filter is approximately 10 feet in diameter by 15 feet tall and contains 32 CERAMEM ceramic filter elements. The ceramic filter elements are cleaned by pulse cleaning using either steam or inert gas. Solids collected in the HEPA filters are discharged through a lock hopper arrangement and collected for final disposal. These solids are similar to the bed solids, except they are much finer, have a lower bulk density, and contain a somewhat higher amount of unreacted carbon.

The HEPA filters are a similar to those used in the PDU. While these filters performed satisfactorily and provide a large filter area in a compact size; alternate configurations need to be evaluated.

Thermal Oxidizer. Hot $\left(900\right.$ to $\left.950^{\circ} \mathrm{F}\right)$ particulate-free gas then passes to the Thermatrix Flameless Thermal Oxidizer system. The hot gas is first cooled by direct water quench and enters the first eductor. The motive fluid for this eductor is a portion of the air required by the thermal oxidizer. Following this eductor, additional dilution air enters the gas prior to entering the 14 feet diameter oxidizer vessel. The oxidizer selected for use in the conceptual facility is a heat recovery model similar to the one used in the PDU. The gas passes up through vertical tubes installed in the ceramic packing where they are preheated to reaction temperature by heat exchange with the hot oxidized gases passing down through the packing. The thermal oxidizer operates at temperatures between 1600 and $1850^{\circ} \mathrm{F}$ at the reaction zone; however, the exhaust exits at about $840^{\circ} \mathrm{F}$ after having exchanged heat with the incoming gas/air mixture.

The oxidizer outlet gas enters a second eductor which is used to maintain a vacuum on the entire destruction system. Steam is used as the motive fluid in this eductor. The gases leaving the eductor are stacked to the atmosphere in the conceptual facility. In the PDU, the oxidizer outlet was quenched and scrubbed to remove acid gases. With the feedstocks selected for this facility, 
no acid gas removal is required. At sites where the wastes have a significant chlorine or sulfur content, a dry scrubbing (quench), packed scrubber combination similar to the PDU configuration would be used to maintain emissions to within acceptable limits while maintaining the facility's zero liquid discharge feature.

The thermal oxidizer selected is a heat recovery model, i.e., the incoming gas is preheated to reaction temperature by heat exchange with the hot oxidized gases as described above. Thermatrix also offers a "once-through" configuration in which there are no heat exchanger tubes within the packing; gas leaves the oxidizer at reaction temperature. This configuration may require the use of a small amount of supplemental fuel, but it would provide the capability to use the hot oxidizer outlet gas for generating superheated steam or other heat recovery applications. The optimum thermal integration of the facility needs to be determined in a more detailed engineering effort.

Utilities. The facility requires superheated steam and compressed air as described above. A package boiler/superheater with a total duty of slightly over 17 million Btu/hr will supply the facility's steam requirements. Compressed air is used as instrument air and as the motive fluid in one of the facilities eductors. A 1100 CFM, 150 psig discharge compressor is required.

In addition, a nitrogen supply system is required to provide inert gas instrument purges and for pulse cleaning of the ceramic HEPA filter elements. A 1500 pound per day liquid nitrogen system is included.

\subsubsection{General Arrangement}

A plan view of the conceptual facility, excluding service utilities, is shown in Figure 7-4. The space requirements are $89^{\prime}$ by $65^{\prime}$. Consistent with the objectives of this preliminary engineering effort, this layout is generic and represents one of many possibilities. Its usefulness is primarily to provide a "feel" for the space requirements. 


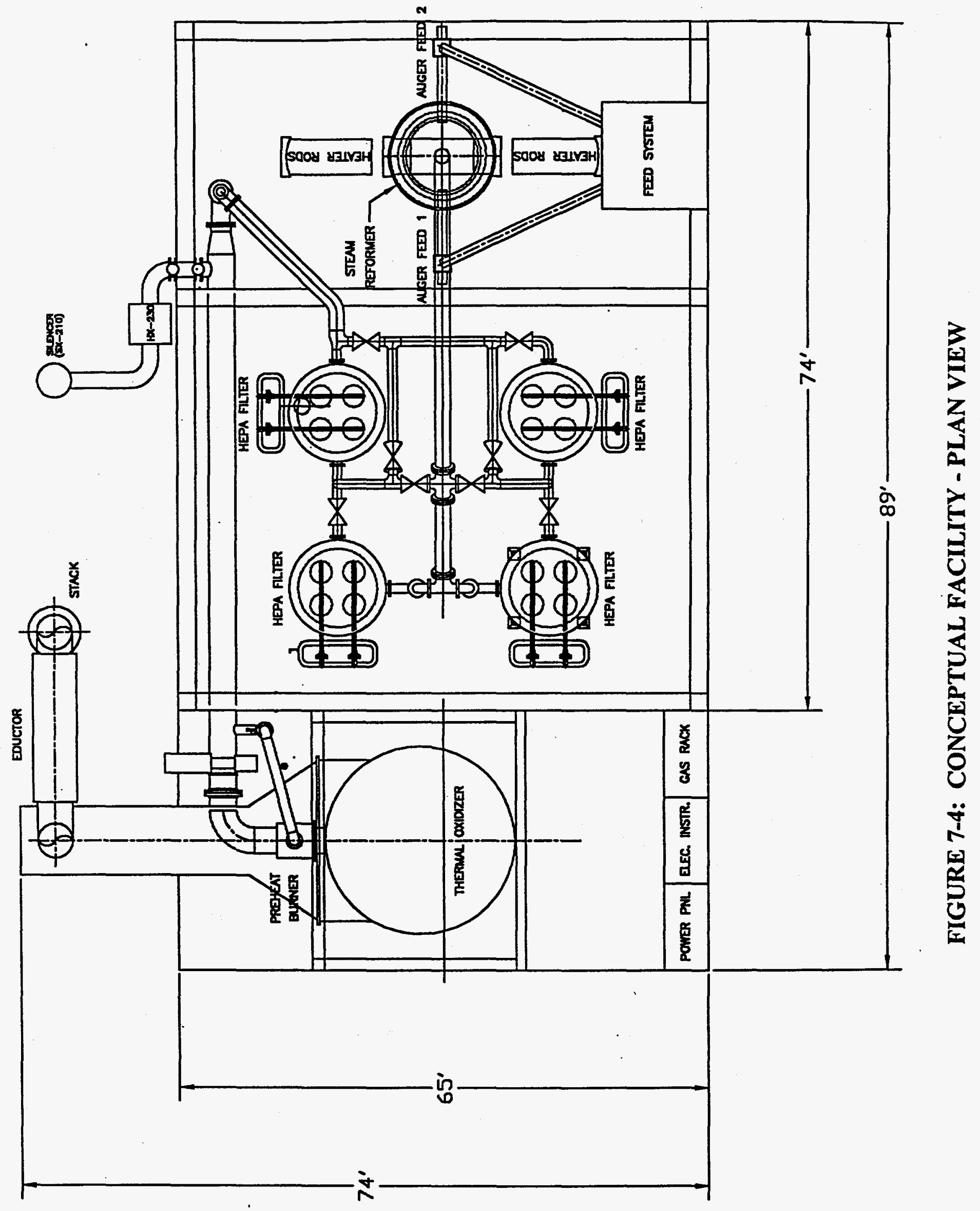


If necessary to meet site requirements, the vessels can be arranged more in-line to minimize the width of the facility. Furthermore, if height requirements permit, a significant amount of floor space can be saved by installing the HEPA filters on more than one floor.

\subsection{POTENTIAL SITES.}

\subsubsection{Sites Considered}

Task 1.6 calls for ThermoChem to review potential sites within the DOE complex as potential demonstration sites for a steam-reforming system in a nominal $500 \mathrm{lb} / \mathrm{hr}$ size. The starting point for this review was the Mixed Waste Inventory Report to identify waste streams applicable to the steam reformer, waste quantities, where the steam reformer would have advantages for DOE over alternatives, and to identify sites where other treatment options were limited. Table 7-1 lists the 18 sites in the Mixed Waste Inventory Report with quantities of LLMW containing greater than 80 percent combustibles. The units are presented in increments of 500 pounds each. For example, Weldon Spring has 1 unit or 500 pounds of LLMW in an oil matrix which has 80 percent or greater combustible organics. ThermoChem looked at the applicability of a 500-pound per hour unit on each of these waste streams. For convenience, the units also reflect the number of hours of operation required for a $500 \mathrm{lb} / \mathrm{hr}$ unit to process the LLMW at the listed site.

The Waste Management Programmatic Environmental Impact Statement (WMPEIS) has addressed these issues as it relates to LLMW in general, not just those applicable to steam reforming. One of the tasks under the WMPEIS evaluates management of approximately 82,000 cubic meters of LLMW currently stored and an estimated 137,000 cubic meters expected to be generated over the next 20 years (excluding LLMW that could be generated as a result of environmental remediation work), for a total of 219,000 cubic meters. 


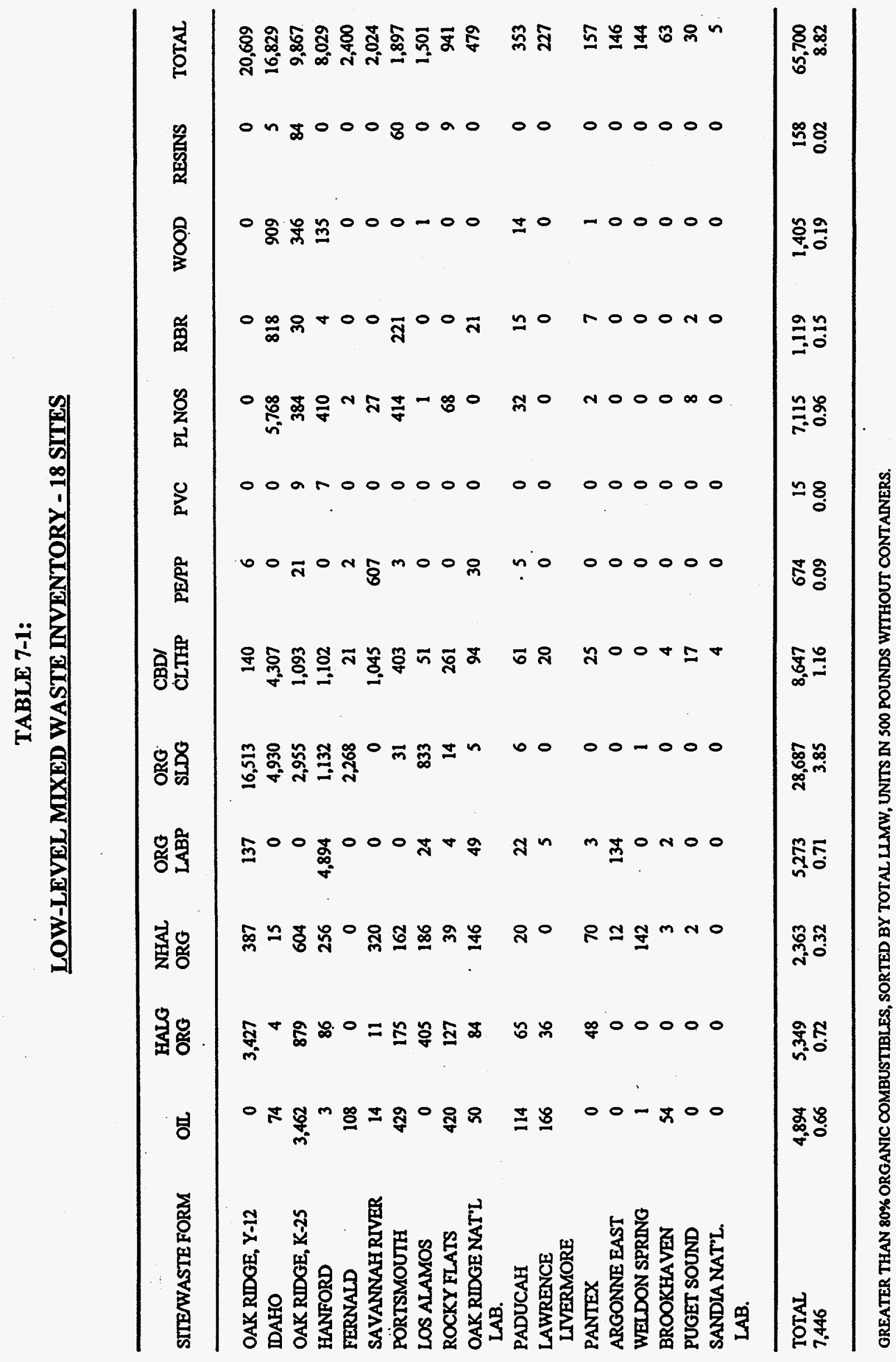


According to the WMPEIS, the DOE-preferred LLMW treatment alternative is for sites that generate a small quantity of LLMW to send it for treatment to one of four large generating sites: Hanford, Idaho National Engineering Laboratory (NREL), Oak Ridge Reservation (ORR), or Savannah River Site (SRS). Eight DOE sites would treat LLMW on-site: Argonne National Laboratory East (ANL-E), Fernal Environmental Management Project (FEMP), Los Alamos and Lawrence Livermore National laboratories (LANL and LLNL), Pantex, Portsmouth Gaseous Diffusion Plant, Rocky Flats Environmental Technology site (RFETS), and Sandia National Laboratory in New Mexico.

DOE'S preferred LLMW disposal alternative is for waste from all the smaller sites to be sent to two or three regional disposal sites which will be selected from the following six sites: Hanford, INEL, LANL, the Nevada Test Site (NTS), ORR and SRS. DOE already has established LLMW or LLW disposal operations at these sites. Four of the six sites (Hanford, INEL, LANL and ORR) already have relatively large volumes of LLMW for disposal. Because these six sites collectively have more than enough capacity for the amount of LLMW DOE needs to dispose, the Department found no need to consider other sites. Furthermore, DOE determined adequate capacity could be provided at a lower cost by using fewer than six sites.

The two Oak Ridge sites, Y-12 and K-25, as shown in Table 7-1, have significant quantities of LLMW applicable to steam reforming. This waste is slated to go to the TSCA Incinerator according to the Oak Ridge Site Treatment Plan. The Idaho Operations Office recently awarded a contract to BNFL for the Advanced Mixed Waste Treatment Facility which is to process 65,000 cubic meters of LLMW at Idaho. The Savannah River site has the Central Incineration Facility (CIF) which will be available for treatment of LLMW with significant quantities of organics.

As noted earlier, the WMPEIS lists the Portsmouth Gaseous Diffusion Plant (PGDP) as a site for an on-site treatment capability. This requirement is made more firm because some of the wastes previously slated to go to the TSCA incinerator may have to be treated at Portsmouth or some other site because of opposition of the State of Tennessee to receive and treat out-of-state 
waste at the TSCA incinerator. A process of elimination of the sites shown in Table 7-1 with quantities of waste applicable to steam reforming that would require at least several months for demonstration testing results with Portsmouth and Fernald as primary sites for further consideration. As noted below, further review has led the ThermoChem team to believe that the LLMW at Portsmouth is more generally applicable to steam reforming and the site represents the first choice for demonstration.

In September 1996, Lockheed Martin Energy Systems, Inc., the managing contractor at PGDP, published a notice seeking expressions of interest in the treatment of a specific PCB- and Uranium-contaminated solid organic waste found at the PGDP. This particular waste is apparently not included in the previously discussed Mixed Waste Inventory Report. The waste, described in greater detail below, exists in approximately 15,712 containers consisting of about 115,000 cubic feet of waste. The existence of this new waste and the excellent applicability of the ThermoChem steam-reforming system, along with the previous review which had made the PGDP a prime candidate for demonstration, led ThermoChem to strongly recommend the Portsmouth Gaseous Diffusion Plant as the selected site for demonstration of the ThermoChem steamreforming system.

In addition, the Paducah Gaseous Diffusion Plant has approximately 3,400 drums, 25,600 cubic feet, with 556,000 pounds of PCB-contaminated absorbents and miscellaneous trash in LLMW waste storage at Paducah. This material could also be restricted from being treated at the TSCA incinerator and could be treated either at the Portsmouth Gaseous Diffusion Plant or a transportable steam reformer could be moved from Portsmouth to Paducah to treat the Paducah waste.

\subsubsection{Selected Site}

The ThermoChem steam-reforming system removes and converts essentially all of the organic material from a LLMW to carbon dioxide and water vapor and isolates all radioactive inorganic material for final disposal. The efficacy of the system is therefore greatest on higher organic content LLMW because of the resulting volume reductions and the system is also very 
effective on LLMW with the most hazardous and toxic material because of its ability to get exceptionally high DREs. A careful review of the LLMW. inventory, treatment capabilities and future needs led ThermoChem to select the Portsmouth Gaseous Diffusion Plant as a site for demonstration of the ThermoChem Steam-Reforming System. Both the PGDP management and the Oak Ridge Operations Office have shown support for such a demonstration. Oak Ridge submitted the ThermoChem Steam-Reforming System for treatment of PGDP LLMW as a proposed project under the recent Technology Deployment Initiative. Copies of their letters of support are provided in Appendix $C$.

After ThermoChem identification of the PCB- and Uranium-contaminated waste at Portsmouth and the potential for significant advantages of steam reforming of this waste, the Federal Energy Technology Center modified the ThermoChem contract to conduct a long-term test on a surrogate of this waste. The origins and nature of this waste found at the Portsmouth Gaseous Diffusion Plant are described below.

The Portsmouth Gaseous Diffusion Plant began operation in 1954 to enrich Uranium for national defense and for commercial nuclear reactors. The U.S. Enrichment Corporation became responsible for enrichment operations in 1993 but the DOE remains responsible for environmental restoration and waste management. The primary contaminants of the LLMW at the PGDP are PCB, TCE and Uranium. Three specific wastes at Portsmouth are of special interest for a steamreforming test and/or commercial treatment because they represent wastes in which Portsmouth has expressed a need for treatment and disposal and because they are very applicable to steamreforming treatment. The first is some 3 million pounds of PCB- and Uranium-contaminated solid waste. The primary source of this waste is the cleanup of oil contaminated with PCB in each of the three process buildings. The original source of the PCB is not certain but most likely it dates back to the construction of the plant when vendors may have saturated flange gaskets with PCBcontaining flame retardant material and glued the gaskets to the metal flanges. Lubricating oil in the exhaust ducts seeped through these gaskets, leaching out PCBs and dripping onto the operating floor and on equipment. Within the three process buildings there were approximately 45,000 joints that had the potential of developing leaks. In an effort to remediate the problem, 
"troughs" had been installed to catch the drips and route them to collection points. The collection points were usually lined with oleophilic absorbent pads.

A variety of absorbent materials have been used to absorb and wipe up the PCB leaks. This contaminated absorbent material collected over the years is now stored as LLMW in some 15,700 barrels and 100 B-25 boxes $\left(4^{\prime} \times 4^{\prime} \times 6^{\prime}\right)$. The PCB contamination levels are from 10 to 500 parts per million by weight (ppm) and the Uranium 235 ranges from 2 to $200 \mathrm{ppm}$. Sorbent Products Company and 3-M Corporation are suppliers of the absorbent pads and sheets of material. The absorbent materials are predominantly polypropylene. The steam reforming of these waste materials will completely isolate the Uranium in a matrix of inorganic residue and totally destroy the PCB and oils and any hazardous organics that may be present. Tests on these absorbent materials have been conducted in the PDU to confirm the ability to shred and feed the material, convert organics to synthesis gas, destroy PCB, and isolate Uranium. The tests were very successful, achieving $99.9999 \%$ DRE of PCB and isolating the Uranium surrogate and achieving up to 400-to-1 volume reduction. The details of this 750 -hour long term test are presented in this report (see Section 6.3).

The other two wastes at PGDP that are of particular interest for treatment in a steamreforming system are spent ion exchange resins and activated carbon, both of which are contaminated with TCE and Uranium. These wastes are the result of a pump-and-treat operation for treatment of contaminated groundwater. The ThermoChem steam-reforming system has shown excellent results on both of these types of feedstocks.

The Portsmouth Gaseous Diffusion Plant is also a recommended site for demonstration or commercial operation of a steam-reforming system because of the availability of an existing building for housing a steam reformer unit. The X-7725 building was built in 1983 to serve as the Recycle/Assembly Building for the Gas Centrifuge Enrichment Plant (GCEP) project. GCEP was intended to replace the gaseous diffusion process but was deemed obsolete in 1985 before installation could be completed. The building consists of five floors with a total of approximately 20 acres of floor space. In order to meet Land Disposal Restrictions, approximately 240,000 
square feet of the facility was converted to a waste storage facility in 1992. Many areas of the building remain unused and could be used for housing a steam reformer system. One particular area in the building that ThermoChem visited that appears to be the most applicable is approximately 55 feet by 24 feet and approximately 77 feet high. Two access balconies are located on three sides of the area and are 20 feet above the ground and 42 feet above the ground, respectively. Dry compressed air, power, water, steam, nitrogen, locker facilities, and office space are located nearby.

A relocatable unit at Portsmouth offers several benefits: a variety of wastes representative of wastes at other sites could be tested, problematic wastes at Piketon could be effectively treated and disposed of, the DOE could verify the readiness of steam-reforming technology for costeffective implementation throughout the DOE complex, and this project and subsequent privatization efforts would reduce the cost and shorten the time for LLMW disposal at Portsmouth and other DOE sites. Another specific attraction for steam-reforming treatment is the potential for Uranium recovery and recycle at Portsmouth. Because of the very high volume reductions (400 to 1), the final and only material exiting a steam reformer system is sand and Uranium compounds. This solid material could possibly be processed at the Uranium Recovery and Recycle facility at the PGDP, thus eliminating the need for any LLW disposal.

ThermoChem is also independently evaluating the potential for construction of a commercial steam-reforming system to be located at the PGDP to process the applicable wastes. 


\section{SECTION 8.0 \\ ECONOMIC EVALUATION/STEAM REFORMING}

In accordance with Tasks 1.6 and 1.7, ThermoChem and its subcontractors have prepared a preliminary cost estimate and economic evaluation for a steam-reforming system with a nominal feed rate of $500 \mathrm{lb} / \mathrm{hr}$. The capital cost, operating cost and all other costs associated with system construction, operation and disposal have been considered and are presented in Section 8.1. A comparison is made with other competing systems in Section 8.2. Also, a comparison between a $500 \mathrm{lb} / \mathrm{hr}$ demonstration unit and a commercial unit is presented in Section 8.3.

\subsection{STEAM REFORMER CAPITAL AND OPERATING COST ESTIMATE}

\subsubsection{Capital Costs}

In accordance with Tasks 1.6 and 1.7, ThermoChem and its subcontractors prepared a preliminary cost estimate for a steam-reforming system with a nominal feed rate of $500 \mathrm{lb} / \mathrm{hr}$ based upon the preliminary conceptual design presented in Section 7.1. The unit is estimated to be capable of processing $500 \mathrm{lb} / \mathrm{hr}$ of a LLMW with organic and moisture content equivalent to a mixed bead ion exchange resin (such as that found at Portsmouth) and $300 \mathrm{lb} / \mathrm{hr}$ of a feed such as the high energy content PCB- and Uranium-contaminated polypropylene absorbent pads found at Portsmouth. As specified in the Statement of Work, the cost estimate includes costs associated with: engineering design, environmental permitting, equipment and subsystem procurement, fabrication and installation, shakedown, and takedown/removal. The cost estimates presented here do not include a contingency but are considered to be plus or minus 15 percent. The equipment costs are based upon written budgetary or final fixed price quotes from vendors. The fabrication and installation costs including piping, structural, electrical, and insulation are based upon vendor quotes for very similar systems constructed or put out to bid by ThermoChem and Stone \& Webster Engineering. Existing ThermoChem, Inc. rates for overhead, fringe benefits, travel, and general and administrative expenses are used. Table 8-1 summarizes the fixed cost estimate. 
TABLE 8-1:

THERMOCHEM STEAM REFORMER CAPITAL COST

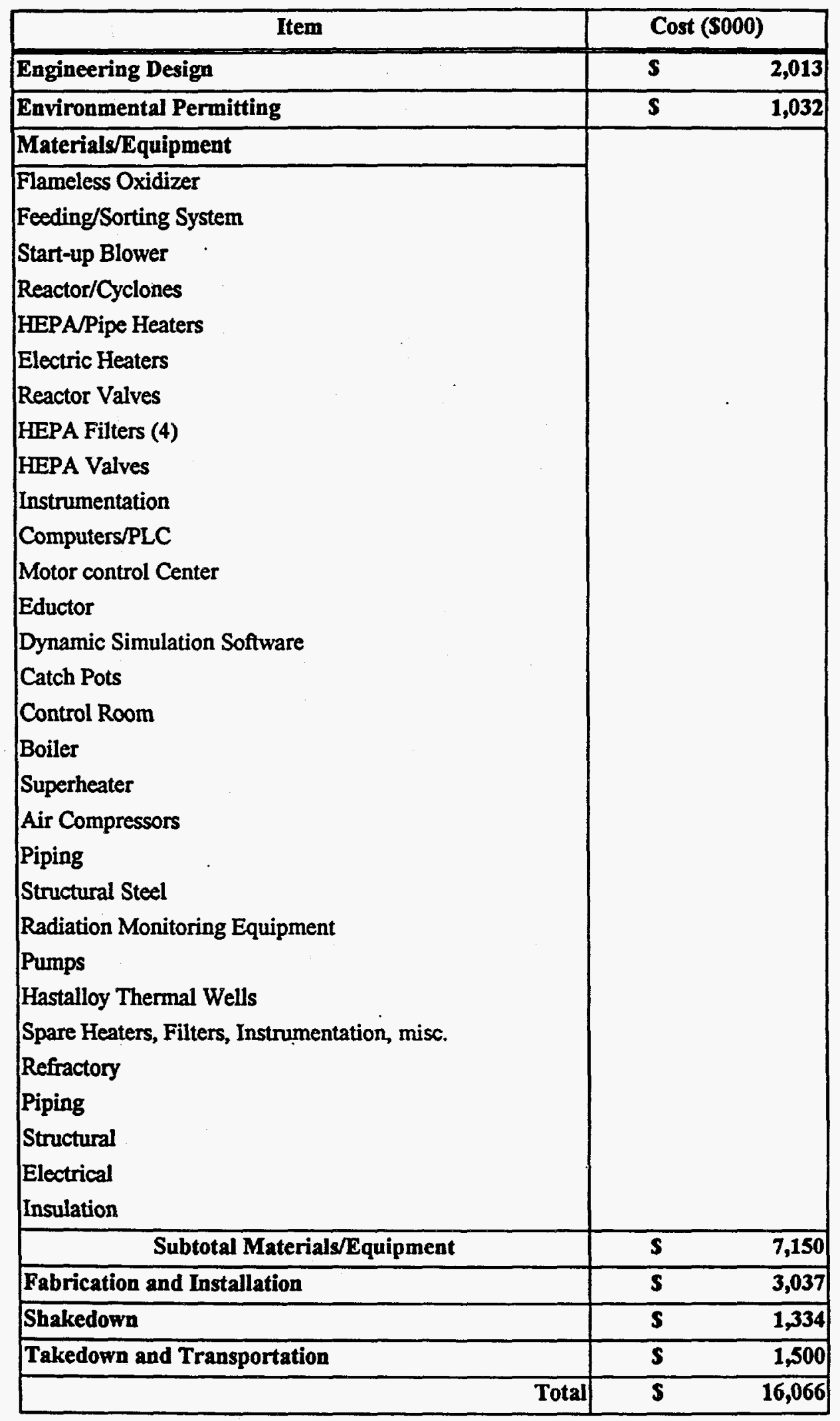


The cost estimates are based upon an eight (8) to ten (10) month period for environmental permitting and analysis. This entire process is considered to be a critical path to installation of a system. The numerous non-incineration permits for commercial Thermatrix units and the intent to recover Uranium for recycle are hoped to facilitate the permitting process. An eight (8) month period is estimated for detailed design and engineering specifications for detailed quotations. A total of twelve (12) months is estimated for fabrication and installation at the Portsmouth site. This is followed by a four (4) month shakedown period. The Thermatrix unit is of a size consistent with existing commercial systems. The steam reformer is being scaled from 100 pounds per hour to 500 pounds per hour for this application. The only real scaling takes place in a modest increase in the diameter of the reactor vessel. It should be noted, however, that a 5,000 pound per hour steam reformer was successfully built and operated on black liquor at a paper mill in North Carolina. The takedown and transportation costs are assumed to include start-up at the new site.

\subsubsection{Steam Reformer Economic Summary}

An economic summary of the preliminary cost per pound of LLMW treated in a ThermoChem steam-reforming system is presented in Table 8-2. The capital cost including takedown and relocation is assumed to be 80 percent financed with 20 percent equity. The amount financed including interest during construction is $\$ 13,548,993$. It is assumed that the equity investor will receive 20 percent return on investment and the financing is 10 percent for 8 years. The preliminary designed presented in Section 7.1 can process $300 \mathrm{lb} / \mathrm{hr}$ of absorbent pads and $500 \mathrm{lbs} / \mathrm{hr}$ of ion exchange resins. The four (4) cases presented vary the feed rate and hours of operation per year from 7,008 to 7,920 .

It is assumed that the facility would run on a continuous, around-the-clock basis. Not counting administration or supervisory personnel, it is assumed that three (3) operators and maintenance personnel will be required per shift. An operator works 40 hours per week but because of holidays, vacation, sick leave, training, etc., an operator's effective average time is $\mathbf{3 4}$ hours per week on a year-round basis. A week is 168 hours, therefore, the number of operators and maintenance personnel is $3 \times(168 / 34)$ or 15 personnel (10 operators plus 5 maintenance/ 
TABLE 8-2:

THERMOCHEM STEAM-REFORMING - ECONOMIC SUMMARY

\begin{tabular}{|c|c|c|c|c|c|c|}
\hline Cost Elements & Case 1 & Case 2 & Case 3 & . Case 4 & & \\
\hline \multicolumn{7}{|l|}{ Capital Recovery } \\
\hline Principal \& Int. per year & $\$ 2,539,678$ & $\$ 2,539,678$ & $\$ 2,539,678$ & $\$ 2,539,678$ & & \\
\hline ROI per year & S 677,450 & $\begin{array}{ll}5 & 677,450\end{array}$ & $\begin{array}{ll}\$ & 677,450\end{array}$ & \$ 677,450 & & \\
\hline Subtotal Capiral & $\$ 3,217,127$ & $\$ 3,217,127$ & $\$ 3,217,127$ & $\$ 3,217,127$ & & \\
\hline \multicolumn{7}{|l|}{ Operating Costs } \\
\hline \multicolumn{7}{|l|}{ Fixed } \\
\hline Labor & & & & & Salary & No. \\
\hline Manager & $\begin{array}{ll}58,000 \\
\end{array}$ & $\begin{array}{ll}S \quad 78,000 \\
\end{array}$ & $\begin{array}{ll} & 78,000\end{array}$ & $\begin{array}{ll}5 & 78,000\end{array}$ & 78,000 & 1 \\
\hline Assistant Manager & $\begin{array}{ll}5 & 75,000\end{array}$ & $\begin{array}{ll}5 & 75,000\end{array}$ & $\$ 75,000$ & $\begin{array}{ll}\mathbf{5} & 75,000\end{array}$ & 75,000 & 1 \\
\hline Operators & $\$ 600,000$ & \$ 600,000 & $\$ 600,000$ & \$ 600,000 & 60,000 & 10 \\
\hline Maintenance & S 250,000 & \$ 250,000 & S 250,000 & S 250,000 & 50,000 & $\overline{5}$ \\
\hline Health \& Safety & S 290,000 & $\$ 290,000$ & $\$ 290,000$ & $\$ 290,000$ & 58,000 & 5 \\
\hline Admin & $\$ \quad 50,000$ & $\$ \quad 50,000$ & $\$ \quad 50,000$ & s 50,000 & 25,000 & 2 \\
\hline Fringe (35\%) & $\$ \quad 470,050$ & $\begin{array}{ll} & 470,050\end{array}$ & $\begin{array}{ll}S & 470,050\end{array}$ & $\$ 470,050$ & & \\
\hline Subtotal Labor & $\$ 1,813,050$ & $\$ 1,813,050$ & $\$ 1,813,050$ & $\$ 1,813,050$ & & \\
\hline Variable Cost & & & & & Use & Units \\
\hline Electricity & S 297,840 & S 336,600 & S 297,840 & S 336,600 & 850 & $k w h / h r$ \\
\hline Propane & 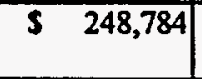 & S 281,160 & \$ 248,784 & \$ 281,160 & & $\begin{array}{l}\text { mmbtul } \\
\mathrm{hr}\end{array}$ \\
\hline Nitrogen & $\mathbf{S}$ & $\mathbf{S}$ & 5 & 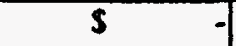 & 0 & cuft/hr \\
\hline Fuel Oil & $\$ 658,913$ & $\$ 744,662$ & \$ 658,913 & S 744,662 & 20.22 & $\begin{array}{l}\text { mmbtw } \\
\mathrm{hr}\end{array}$ \\
\hline Air Emissions Sampling & \$ $\quad 120,000$ & $\$ \quad 120,000$ & $\begin{array}{ll}\$ & 120,000\end{array}$ & $\begin{array}{ll}\$ & 120,000\end{array}$ & & \\
\hline Solid Sampling & S 120,000 & $\begin{array}{ll}\$ & 120,000\end{array}$ & $\begin{array}{ll}\$ & 120,000\end{array}$ & $\begin{array}{ll}\$ 120,000 \\
\end{array}$ & & \\
\hline Maintenance Supplies & $\begin{array}{ll}S & 120,000\end{array}$ & $\begin{array}{ll}S & 120,000\end{array}$ & \$ 120,000 & $\$ 120,000$ & & \\
\hline Overhead* & $\begin{array}{ll}\$ & 675,717\end{array}$ & $\begin{array}{ll}\text { S } 707,094 \\
\end{array}$ & $\$ 675,717$ & $\begin{array}{ll}\$ & 707,094 \\
\end{array}$ & $20 \%$ & \\
\hline Subtotal Variable & S $2,241,255$ & S $2,429,517$ & S $2,241,255$ & $\$ 2,429,517$ & & \\
\hline Total Cost per year & \$ 7,271,432 & $\$ 7,459,694$ & $\$ 7,271,432$ & $\$ 7,459,694$ & & \\
\hline Pounds Processed & $2,102,400$ & $2,376,000$ & $3,504,000$ & $3,960,000$ & & \\
\hline Cost per Pound & 3.46 & 3.14 & 2.08 & .1 .88 & & \\
\hline Disposal Cost ${ }^{(1)}$ & 0.17 & 0.17 & 0.67 & 0.67 & & \\
\hline & Case 1 & Case 2 & Case 3 & Case 4 & & \\
\hline Throughput, lbs/hr & 300 & 300 & 500 & 500 & & \\
\hline Availability, \% & $80 \%$ & $90 \%$ & $80 \%$ & $90 \%$ & & \\
\hline Hours Operation/Year & 7008 & 7920 & 7008 & 7920 & & \\
\hline Feed Material & abs. pads & abs. pads & resin & resin & & \\
\hline
\end{tabular}

- Includes insurance, administration, telephone, etc.

(1) If applicable. 
TABLE 8-2:

THERMOCHEM STEAM-REFORMING - ECONOMIC SUMMARY

(Continued)

\begin{tabular}{|c|c|c|c|c|c|}
\hline Financial Terms & & Variable C & & & \\
\hline Interest Rate & $10 \%$ & Electricity & $\mathbf{s}$ & 0.05 & S/kwhr \\
\hline Construction Period & $12 \mathrm{mo}$. & Propane $^{1}$ & 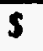 & 7.10 & S/mmbtu \\
\hline Capital Cost, $\$$ & $\$ 16,066,000$ & Nitrogen & $\$$ & 0.09 & \$/cuft \\
\hline Interest During Constr. & $\$ \quad 870,242$ & Fuel Oil & $\$$ & 4.65 & $\$ / m m b t u$ \\
\hline Percent Financed & $80 \%$ & & & & \\
\hline Amount Financed,s & $\$ 13,548,993$ & & & & \\
\hline Loan Duration, years & 8 & & & & \\
\hline Equity ROI & $20 \%$ & & & & \\
\hline
\end{tabular}

1) Propane is for start-up only.

\section{Disposal Costs}

\begin{tabular}{|c|c|c|c|c|c|c|}
\hline Input & Density & & Volume & & Weight & \\
\hline Polypropylene pads & 6 & $\mathrm{lb} /$ cuft & 0.167 & cuft & 1 & Ib \\
\hline Ion Exchange Resin & 40 & $\mathrm{lb} /$ cuft & 0.025 & cuft & 1 & $\overline{\mathrm{Ib}}$ \\
\hline \multicolumn{7}{|l|}{ Output } \\
\hline Polypropylene pads (400:1) & 100 & $\mathrm{lb} /$ cuft & 0.00041667 & cuft & 0.04167 & lb \\
\hline Ion Exchange Resin (15:1) & 100 & lb/cuft & 0.00166667 & cuft & 0.16667 & 16 \\
\hline Disposal Cost & \$/cuft & Per Pound of Input & & & & \\
\hline Polypropylene pads & 400 & 0.17 & & & & \\
\hline Ion Exchange Resin & 400 & $\begin{array}{ll} & 0.67\end{array}$ & . & & & \\
\hline
\end{tabular}


operators). An additional 5 personnel (one per shift) are provided for environmental and personnel safety regulations. A fringe labor rate of 35 percent is provided for unemployment, social security taxes, vacation time, holidays, and sick time.

The variable operating costs are for electricity, propane for start-up of the flameless thermal oxidizer, and fuel oil for the boiler. It is assumed that steam will be used for instrumentation purge so no nitrogen will be consumed. The quantities consumed per hour are based upon the mass and energy balance presented for the preliminary design in Section 7.1. The electricity, propane and fuel oil rates are based upon quotes from a mid-USA site.

In addition to the normal operation and monitoring conducted by operating personnel using the continuous emissions monitoring system, it is assumed that outside laboratory testing will be required on solid and gas samples. The solid samples would be the solid bed material extracted from the reactor and HEPA filters. The gas samples are from stack emissions. It is important to note that the ThermoChem steam-reforming system has no liquid effluent. The maintenance supplies are assumed to be $\$ 10,000$ per month in each case. It is anticipated that a very small number of major items would need replacement during an expected 15 to 20 year life of the system. Screw feeder motors and seals would need maintenance and lock hopper valves may need replacement once or twice during a plant lifetime. Refractory may need replacement but the alternate approach of using a stainless steel reactor vessel with insulation on the exterior would preclude this maintenance cost.

The testing under this DOE contract has confirmed a volume reduction of 400 to 1 and as high as 1,000 to 1 for the absorbent pads and 15 to 1 for spent ion exchange resin. For a demonstration or commercial operation at the Portsmouth Gaseous Diffusion Plant, it is assumed that the solid material containing Uranium, sand and carbon particles will be returned to the recycle facility for recovery of the Uranium. The costs presented in Table 8-2 do not include costs for the recovery process. If the material must be disposed of off-site, it is estimated that the disposal cost would be $\$ .17$ and $\$ .67$ per pound of input for pads and resin, respectively, assuming $\$ 400 /$ cu.ft. disposal costs. The data for this analysis is shown in Table 8-2. 
As shown in Table 82, the estimated cost per pound for treating ion exchange resins or polypropylene pads under the four cases ranges from $\$ 1.88 / \mathrm{lb}$ to $\$ 3.46 / \mathrm{b}$. This is based on a $80 / 20$ debt-to-equity ratio and a $20 \%$ Return on Investment for the equity holder. Twenty percent is considered an acceptable ROI for an industrial process. In the case of the processing of LLMW, as shown in Section 8.2, the TSCA Incinerator in Oak Ridge, Tennessee costs approximately $\$ 6.00$ to $\$ 8.00$ per pound in operating costs only, without costs for capital recovery. The steam-reforming costs per pound with capital recovery are $75 \%$ to $35 \%$ lower than the TSCA Incinerator costs without capital recovery. If a commercial steam-reforming system owner/operator were to be paid a processing fee comparable to the TSCA Incinerator costs, the ROI would significantly exceed $20 \%$.

\subsection{COMPETING SYSTEMS ECONOMICS}

The U.S. Department of Energy (DOE) operates a rotary kiln incinerator with an after burner at the K-25 Site in Oak Ridge, Tennessee. The K-25 Site Toxic Substance Control Act (TSCA) Incinerator is unique in that it is both permitted as a Resource Conservation and Recovery Act (RCRA) treatment facility and authorized by the U.S. Environmental Protection Agency (EPA) to treat TSCA wastes. Approximately $7 \times 10^{6} \mathrm{~kg}$ of liquid wastes, mainly wastes contaminated with Uranium and Polychlorinated Biphenyls (PCBs), have been treated since TSCA operations began in 1991. These wastes are defined as mixed wastes because they contain both radioactive and hazardous constituents. The incinerator thermal capacity is about $8.8 \mathrm{MW}$ $\left(3.0 \times 10^{7} \mathrm{Btu} / \mathrm{hr}\right)$.

Liquid wastes are transported to the K-25 Site in tank trucks from seven sites located in Tennessee, Kentucky, and Ohio. These sites are the former gaseous diffusion plant (GDP) at K25, the GDPs at Portsmouth and Paducah, the Oak Ridge National Laboratory (ORNL), the Y-12 Plant, the Reactive Metals, Inc. (RMI) Extrusion Plant and the Fernald Environmental Management Project. The wastes are blended in a $3.41 \times 10^{5}-\mathrm{L}\left(90.1 \times 10^{3}\right.$ gal) tank farm (15 tanks) to optimize combustion efficiency and feed rate within permitted limits. 
Treatment of liquid wastes including waste oils, solvents and water solutions began in 1991 when $1 \times 10^{6} \mathrm{~kg}$ of waste was incinerated. Since 1991, the throughput of the incinerator has increased every year to $1.8 \times 10^{6} \mathrm{~kg}\left(4 \times 10^{6} \mathrm{lb}\right)$ incinerated in 1994. DOE plans to shift the operation to solid waste treatment. Currently, only waste from the seven sites previously mentioned are being treated at the TSCA Incinerator; however, DOE is considering the use of the incinerator to treat RCRA and TSCA waste streams from other DOE sites.

The total annual operating cost of the TSCA Incinerator is about \$25 million. This cost can be broken down into eight functional areas: plant operations, sampling and analysis, monitoring, maintenance, program management and support, administrative, environmental compliance, and technical support. These costs have previously been studied in some detail. At an annual operating budget of $\$ 25,000,000$ with a throughput of 3 to 4 million pounds per year, the cost per pound at the TSCA incinerator is approximately $\$ 6$ to $\$ 8$ per pound of material processed. This does not include transportation costs to the site or capital recovery or final waste disposal costs. ${ }^{(8)}$

The U.S. Department of Energy's Mixed Waste Focus Area (MWFA) ${ }^{(9)}$ commissioned an evaluation of mixed waste treatment technologies that are alternatives to incineration for destruction of hazardous organic wastes. The purpose of this effort was to evaluate technologies that are alternatives to open-flame, free-oxygen combustion (as exemplified by incinerators), and recommend to the Waste Type Managers and the MWFA which technologies should be considered for further development. Alternative technologies were defined as those that have the potential to destroy organic material without the use of open-flame reactions with free gas-phase oxygen as the reaction mechanism, reduce the off-gas volume and associated contaminants (metals, radionuclides, and particulates) emitted under normal operating conditions, eliminate or reduce the production of dioxins and furans, and reduce the potential for excursions in the process that can lead to accidental release of harmful levels of chemical or radioactive materials. Twentythree technologies were identified that have the potential for meeting these requirements. These technologies were rated against the categories of performance, readiness for deployment, and environment, safety and health. The top ten technologies that resulted from this evaluation are 
Steam Reforming, Electron Beam, UV Photo-Oxidation, Ultrasonics, Eco Logic reduction process, Supercritical Water Oxidation, Cerium Mediated Electrochemical Oxidation, DETOX $^{(\mathrm{SM})}$, Direct Chemical Oxidation (peroxydisulfate), and Neutralization/Hydrolysis.

\subsection{DEMONSTRATION/COMMERCIAL UNIT COMPARISON}

The entire DOE LLMW inventory is approximately 160,000 tons of material or approximately 320 million pounds. Assuming 5 years of operation at 85 percent availability, one treatment unit of approximately 4 tons per hour capacity would treat all of the LLMW in the DOE inventory. For many reasons this is not practical but it places an upper limit on the size of a commercial unit. A review of the LLMW inventory at various DOE sites as presented in Table 71 indicates that the demonstration scale of $500 \mathrm{lb} / \mathrm{hr}$ is indeed a very appropriate size for a commercial unit.

The ThermoChem steam-reforming system would be significantly scaled down in size from the paper industry application to a LLMW application. Commercial units for processing black liquor are typically 12 to 30 tons per hour of dry black liquor solids. As in the paper industry, the scale is driven by the site needs of the customer. The scale of a commercial LLMW treatment unit is driven primarily by the availability of adequate quantities of LLMW for processing at a DOE site. The $500 \mathrm{lb} / \mathrm{hr}$ scale is considered a viable commercial scale even when considering sites with larger quantities of waste. As shown by the data in Table 7-1, at the site with the largest quantity of LLMW applicable to steam reforming, a $500 \mathrm{lb} / \mathrm{hr}$ unit would require about three years of nominal operation to process all of the waste at the site. Even for sites that might be designated as a regional treatment center, a $500 \mathrm{lb} / \mathrm{hr}$ unit or slightly larger is considered commercial scale. 


\section{REFERENCES}

1) Department of Energy Mixed Waste Inventory Report, October 1995.

2) Flameless Thermal Oxidation, Innovative Technology Summary Report, demonstrated at M Area, Savannah River site, U.S. Department of Energy, September 1995.

3) Treatability Study for Thermatrix Flameless Thermal Oxidation, Radian Corporation, January 1996.

4) Field Demonstration of a Full-Scale In-Situ Thermal Desorption System for the Remediation of Soil Containing PCBs and Other Hydrocarbons, HazWaste World/ Superfund XVII, October 1996.

5) Flashback Testing of Thermatrix ES-60H Oxidizer, Fenwal Safety Systems, December 1992.

6) Mixed Waste Characterization, Treatment Disposal Focus Area, Technology Summary, DOE/EM-0293, prepared by the Office of Science and Technology, DOE, August 1996.

7) Schwinkendorf, W.E., "Current and Innovative Technologies for Treatment of Mixed Low-Level Waste," Management of Mixed Radioactive and Hazardous Waste, ASME Short Course, Baltimore, Maryland, November 4-8, 1996.

8) Loghry, Steve L., R. Salmon, W.H. Hermes, "Analysis of Operating Costs of a Low-Level Mixed Waste Incineration Facility," Oak Ridge National Laboratory, Oak Ridge, Tennessee, 1996.

9) Schwinkendorf, W.E., B.C. Musgrave, R.N. Drake, "Evaluation of Alternative Nonflame Technologies for Destruction of Hazardous Organic Waste," prepared for the U.S. Department of Energy/Assistant Secretary for Environmental Management, DOE Idaho Operations Office, by Idaho National Engineering Laboratory, Mixed Waste Focus Area, Lockheed Martin Idaho Technologies Company, Idaho Falls, Idaho, April 1997.

10) "Recipes of Surrogate Formulations for Thermal Treatment of Low-Level Mixed Waste," Mixed Waste Integrated Program Office of Oak Ridge National Laboratory for the U.S. Department of Energy under Contract No. DE-AC05-840R21400, Document No. DOE/MWIP-16, January 1994. 


\section{ACRONYMS}

\begin{tabular}{ll} 
CEM & Continuous Emission Monitoring \\
DRE & Destruction Removal Efficiency \\
FTO & Flameless Thermal Oxidizer \\
HEPA & High Efficiency Particulate \\
GCMS & Gas Chromatograph/Mass Spectrometer \\
ID & Induced Draft \\
LLMW & Low-Level Mixed Waste \\
LT HEPA & Low-Temperature, High Efficiency Particulate \\
MTCI & Manufacturing and Technology Conversion International, Inc. \\
PCB & Poly-Chlorinated BiPhenol \\
PDU & Process Development Unit \\
PGDP & Portsmouth Gaseous Diffusion Plant \\
PLC & Programmable Logic Controller \\
POHC & Principal Organic Hazardous Constituents \\
RCRA & Resource Conservation and Recovery Act \\
TCE & Tri Chloro Ethylene \\
TSCA & Toxic Substances Control Act \\
\hline
\end{tabular}




\section{APPENDIX A:}

\section{SURROGATE FEEDSTOCK IDENTIFICATION AND PREPARATION}




\section{APPENDIX A: \\ SURROGATE FEEDSTOCK IDENTIFICATION AND PREPARATION}

\section{BACKGROUND}

A diverse spectrum of waste streams exist at various Department of Energy (DOE) sites. The wastes include combustible liquids and solids and noncombustible contaminated solids such as concrete and vermiculite. Some of the combustible material are halogenated hydrocarbons such as dichlorobenzene or polyvinylchloride (PVC) which are difficult to treat in most systems; the PVC could be in the form of gloves as found at a number of DOE installations. The waste frequently contains radionuclides, a small percentage of activated carbon, and an ion exchange resin. Typically, the wastes are classified into seven types as listed below:

- Heterogeneous debris;

- Aqueous halogenated organic liquids;

- Absorbed aqueous organic liquids;

- High organic content sludge;

- Cement, sludge, ash, and solids;

- Natural aqueous waste; and

- Piketon Waste.

Based on the document (DOE/MWIP-16) published by the Mixed Waste Integrated Program Office of Oak Ridge National Laboratory ${ }^{(10)}$, six surrogate feedstocks representing the six waste types in the DOE inventory were suggested for use in testing by Sandia National Laboratory staff and endorsed by staff from the Idaho National Engineering Laboratory.

A survey of LLMW at DOE sites also resulted in the identification of a large inventory of PolyChlorinated BiPhenyl (PCB) solid waste at the Portsmouth Gaseous Diffusion Plant as a desirable feed for further tests. About 20 to 25 percent of the DOE LLMW inventory includes halogenated organics such as PCB. The PCB-contaminated waste at Portsmouth is now contained in approximately 15,000 barrels and amounts to about 3 million pounds. This waste 
was previously planned for destruction at the TSCA incinerator but is not now scheduled for processing until well into the 21 st century. No other options appear to be available or planned for this waste. It is definitely in the DOE interest to further the development and testing of the ThermoChem system and accelerate its commercial deployment. The next logical step toward meeting that objective is to conduct detailed and longer term tests on representative materials using hazardous compounds and radioactive surrogates. The PCB contaminated waste at Portsmouth represents a good choice because of the large quantities at Portsmouth and other sites and the lack of other treatment options. Since several DOE LLMW materials also contain mercury compounds, a surrogate test on a material containing mercury is also needed. Mercury was not included in the original surrogates to be tested under the DOE contract. These considerations led to the selection of a seventh feedstock.

The task schedule calls for preparing all the surrogate wastes prior to the start of the PDU test program. Each feedstock is to be prepared for twice the anticipated test quantity. After review of the surrogate feedstock requirements and the schedule duration, the feedstock will be made up prior to use in batches of sufficient quantity to assure that the PDU test program meets the objectives of the contract.

An independent laboratory will either certify the analysis of the surrogate feedstock or provide an audit that the feedstock was prepared in accordance with the approved procedure.

The following sections describe the seven surrogate feedstocks and the approved mixing instructions.

\section{SURROGATE FEEDSTOCK FORMULATIONS}

Sandia National Laboratory provided MTCI with excerpts from the document DOE/ MWIP-16 for the six formulations listed in Table A-1. All the surrogates contain RCRA organics (volatile and semi-volatile), surrogates of radionuclides, RCRA metals, and bulk ingredients. 
TABLE: A-1:

SURROGATE FORMULATION - SUMMARY

FORMULATION NO.:

1

2

3

4

5

6

\begin{tabular}{|c|c|c|c|c|c|c|}
\hline 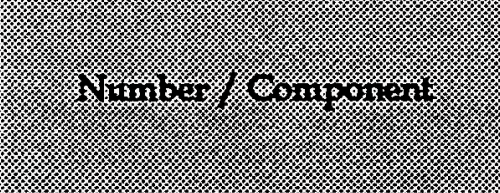 & 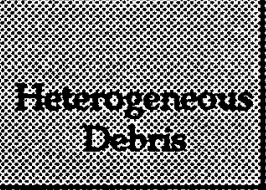 & 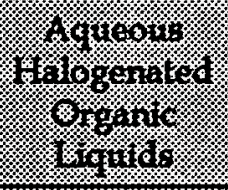 & 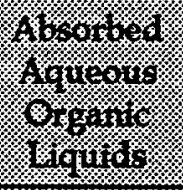 & 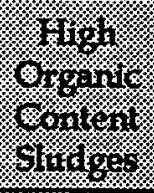 & 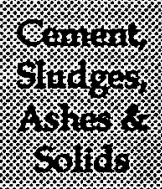 & 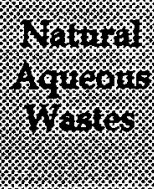 \\
\hline & / & W & 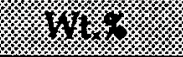 & 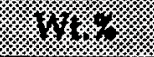 & w/ & wy \\
\hline $\begin{array}{l}\text { Bulk Ingredient } \\
\text { 1. Activated Carbon }\end{array}$ & 5. & 5. & 5. & 10. & 10. & 5. \\
\hline 2. Cation Exchange Resin & 5. & 5. & 5. & 5. & 5. & 5. \\
\hline 3. Water & 10. & 10. & 19. & 15. & 30. & 75. \\
\hline 4. Wood & 10. & & & & & \\
\hline 5. Polyvinylchloride (PVC) & 10. & & & & & \\
\hline 6. Neoprene & 10. & . & & & & \\
\hline $\begin{array}{l}\text { 7. Mild } \\
\text { Steel/Hematite } / \mathrm{Fe}_{2} \mathrm{O}_{3}\end{array}$ & 10. & & & 10. & 3. & \\
\hline 8. Glass Beads & 10. & & & & & \\
\hline 9. Cement/Concrete & 8. & & & & & \\
\hline 10. Alumina $/ \mathrm{Al}_{2} \mathrm{O}_{3}$ & 10. & & & 5. & 2. & \\
\hline 11. Diatomaceous Earth & 10. & & & & & \\
\hline 12. Toluene & & 10. & & & & \\
\hline 13. Tetrachloroethylene & & 10. & & & & \\
\hline 14. Mineral Oil & & 10. & & 7. & & \\
\hline 15. Ethylene Glycol & & 10. & 10. & 10. & & \\
\hline 16. Vermiculite & & 19. & 25. & & & \\
\hline 17. Perlite $\left(\mathrm{SiO}_{4}\right)$ & & & 25. & 10. & 10. & \\
\hline $\begin{array}{l}\text { 18. } \mathrm{CaSO}_{4} \cdot 2 \mathrm{H}_{2} \mathrm{O} / \text { Plaster of } \\
\text { Paris }\end{array}$ & & & & 10. & 3. & \\
\hline 19. Phenol & & & & 10. & & \\
\hline 20. Fly Ash (ASTM Class F) & & & & & 15. & 1. \\
\hline $\begin{array}{l}\text { 21. Concrete (cured, crushed, } \\
\text { screened) }\end{array}$ & & & & & 20. & \\
\hline
\end{tabular}




\section{TABLE A-1: \\ SURROGATE FORMULATION - SUMMARY \\ (Cont'd)}

\begin{tabular}{|c|c|c|c|c|c|c|}
\hline FORMULATION NO.: & 1 & 2 & 3 & 4 & 5 & 6 \\
\hline \multirow[t]{2}{*}{ 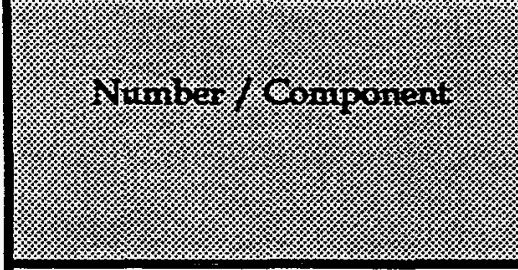 } & 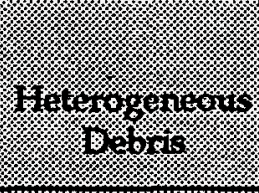 & 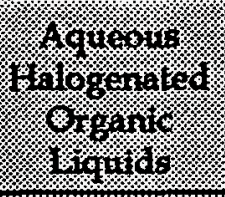 & 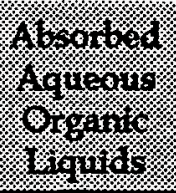 & 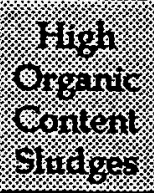 & 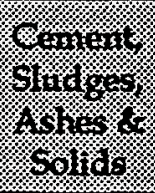 & 䇣y \\
\hline & 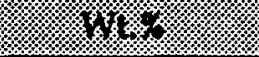 & 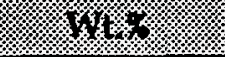 & 4 & 4 & (n) & (x) \\
\hline 22. $\mathrm{CaCl}_{2}$ & & & & & & 3. \\
\hline 23. $\mathrm{NaHCO} / \mathrm{NaNO}_{3}$ * & & & & & & 3. \\
\hline 24. $\mathrm{MgSO}_{4} \cdot 7 \mathrm{H}_{2} \mathrm{O} / \mathrm{Al}\left(\mathrm{NO}_{3}\right)_{3}$ " & & & & & & 3. \\
\hline 25. $\mathrm{Na}_{2} \mathrm{HPO}_{4} \bullet 7 \mathrm{H}_{2} \mathrm{O}$ & & & & & & 3. \\
\hline $\begin{array}{l}\text { RCRA Metals } \\
\text { 26. } \mathrm{Cr}\left(\mathrm{NO}_{3}\right)_{2} \bullet 6 \mathrm{H}_{2} \mathrm{O} \text { (or oxide) }\end{array}$ & 0.1 & 0.1 & 0.1 & 0.1 & 0.1 & 0.1 \\
\hline 27. $\mathrm{Ni}\left(\mathrm{NO}_{3}\right)_{2} \bullet 6 \mathrm{H}_{2} \mathrm{O}$ & 0.1 & 0.1 & 0.1 & 0.1 & 0.1 & 0.1 \\
\hline 28. $\mathrm{Pb}\left(\mathrm{NO}_{3}\right)_{2}$ & 0.1 & 0.1 & 0.1 & 0.1 & 0.1 & 0.1 \\
\hline 29. $\mathrm{Cd}\left(\mathrm{NO}_{3}\right)_{2} \bullet 4 \mathrm{H}_{2} \mathrm{O}$ & 0.1 & 0.1 & 0.1 & 0.1 & 0.1 & 0.1 \\
\hline $\begin{array}{l}\text { RCRA Organics } \\
\text { 30. Naphthalene }\left(\mathrm{C}_{10} \mathrm{H}_{8}\right)\end{array}$ & 0.5 & 10. & 5. & 2. & 0.5 & 0.5 \\
\hline $\begin{array}{l}\text { 31. 1,2-Dichlorobenzene } \\
\left(\mathrm{C}_{6} \mathrm{H}_{4} \mathrm{Cl}_{2}\right)\end{array}$ & 0.5 & 10. & 5. & 5. & 0.5 & 0.5 \\
\hline $\begin{array}{l}\text { Radionuclide Surrogate } \\
\text { 32. } \mathrm{CeCl}_{3}\end{array}$ & 0.3 & 0.3 & 0.3 & 0.3 & 0.3 & 0.3 \\
\hline 33. $\mathrm{CsCl} / \mathrm{CsNO}_{3}$ & 0.3 & 0.3 & 0.3 & 0.3 & 0.3 & 0.3 \\
\hline
\end{tabular}

* Suggested substitute for surrogate Feedstock No. 6 only. 


\section{Surrogate Formulation No. 1: Heterogeneous Debris}

Formulation No. 1 is heterogeneous debris materials which contain 50 percent combustibles. It was referred by the MWIP report mentioned above as MWTP-5400, patterned after buried waste and EPA treatability group \#13 for solid debris waste that exhibit the characteristics of toxicity due to metals (EPA Waste Codes D004 - D011) with non-friable, inorganic matrices such as glass, concrete, refractory brick, slag, etc.

Table A-2 lists the ingredients and Table A-3 gives the mixing instructions. It is proposed that mild steel may be substituted by iron ore (hematite) for ease of size reduction. It is possible to remove large mild steel pieces magnetically and clean them by sand-blasting or other means instead of steam reforming.

Naphthalene and 1,2-Dichlorobenzene are chosen to represent semi-volatile organic compounds that are refractory to thermal destruction. Cesium and cerium chlorides at $0.3 \mathrm{wt} . \%$ are chosen as volatile radionuclide surrogates, in place of technicium, etc. Trace toxic metal nitrates - chromium, nickel, lead and cadmium - are added at $0.1 \mathrm{wt} . \%$. Other bulk combustibles include wood chips, PVC, neoprene, activated carbon and cation exchange resin. Glass, concrete/cement, alumina and diatomaceous earth, along with the hematite, represent the noncombustible debris.

\section{Surrogate Formulation No. 2: Aqueous Halogenated Organic Liquids}

This formulation, referred to as MWTP-2110, consists of a mixture of aqueous and halogenated organic liquid waste corresponding to EPA Waste Codes F001, F002, F005, D004, and D005 - D010.

The surrogates selected are a combination of common halogenated and RCRA organic compounds (up to $70 \%$ combustibles) that have high vapor pressures and are challenging incinerability indices. These include tetrachloroethylene, ethylene glycol, toluene, naphthalene, 
TABLE A-2:

SURROGATE FORMULATION NO. 1

MWTP 5400 - HETEROGENEOUS DEBRIS

\begin{tabular}{|c|c|c|}
\hline No. & COMPONENT NAME & $w \hbar \%$ \\
\hline \multicolumn{3}{|c|}{ Bulk Ingredient } \\
\hline 1 & Activated carbon & 5 \\
\hline 2 & Cation exchange resin & 5 \\
\hline 3 & Water & 10 \\
\hline 4 & Wood & 10 \\
\hline 5 & Polyvinylchloride (PVC) & 10 \\
\hline 6 & Neoprene & 10 \\
\hline 7 & Mild steel/ $/ \mathrm{Fe}_{2} \mathrm{O}_{3}$ & 10 \\
\hline 8 & Glass beads & 10 \\
\hline 9 & Cement & 8 \\
\hline 10 & Alumina & 10 \\
\hline 11 & Diatomaceous earth & 10 \\
\hline \multicolumn{3}{|c|}{ RCRA Metals } \\
\hline 12 & $\mathrm{Cr}\left(\mathrm{NO}_{3}\right)_{2} \bullet 6 \mathrm{H}_{2} \mathrm{O}$ & 0.1 \\
\hline 13 & $\mathrm{Ni}\left(\mathrm{NO}_{3}\right)_{2} \cdot 6 \mathrm{H}_{2} \mathrm{O}$ & 0.1 \\
\hline 14 & $\mathrm{~Pb}\left(\mathrm{NO}_{3}\right)_{2}$ & 0.1 \\
\hline 15 & $\mathrm{Cd}\left(\mathrm{NO}_{3}\right)_{2} * 4 \mathrm{H}_{2} \mathrm{O}$ & 0.1 \\
\hline \multicolumn{3}{|c|}{ RCRA Organics } \\
\hline 16 & Naphthalene $\left(\mathrm{C}_{10} \mathrm{H}_{8}\right)$ & 0.5 \\
\hline 17 & 1,2-Dichlorobenzene $\left(\mathrm{C}_{6} \mathrm{H}_{4} \mathrm{Cl}_{2}\right)$ & 0.5 \\
\hline \multicolumn{3}{|c|}{ Radionuclides } \\
\hline 18 & $\mathrm{CeCl}_{3}$ & 0.3 \\
\hline 19 & $\mathrm{CsCl}$ & 0.3 \\
\hline
\end{tabular}


TABLE A-3:

MIXING INSTRUCTIONS FOR FORMULATION NO. 1

\section{MWTP 5400 - HETEROGENEOUS DEBRIS}

STEPNO.

SEQUENCE/INSTRUCTIONS

1. Weigh drum and lid and record.

2. Add constituents \#4 through \#11 to drum.

3. Activate drum mixer for $\geq 5$ minutes.

4. Weigh drum, lid and mixture and record.

5. In any sequence, carefully add all of each of the 4 RCRA metal compounds (\#12$\# 15)$ and 2 rad surrogate salts (\#18-\#19) to a separate container. Add enough of \#3 (water) to dissolve.

6. Add $100 \%$ of the cation exchange resin (\#2) to the container (Step 5) and stir.

7. Add the RCRA/RAD metals/resin slurry prepared in Steps 5-6 to drum. Rinse the drum with any excess water (\#3) and add to drum.

8. Activate drum mixer for $\geq 5$ minutes.

9. Weigh drum, lid and mixture and record.

10. Carefully add the RCRA organics (\#16-\#17) to a separate container. Mix the two components until they are equally intermixed.

11. Slowly add, with constant stirring, the activated carbon (\#1) into the container holding the RCRA organic (\#16-\#17) mixture. Add enough of the activate carbon to absorb the organic liquids.

12. Add the remaining activated carbon not used in Step 11 and the mixture from the container (Steps 10-11) into the drum. Seal the drum.

13. Activate drum mixer for $\geq 15$ minutes.

14. Weigh drum, lid and surrogate waste stream mixture and record.

15. Measure drum fill level and calculate drum fill capacity. 
1,2-Dichlorobenzene, and mineral oil. Bulk ingredients are activated carbon, cation exchange resin, and vermiculite. Same levels of RCRA metal nitrates and radionuclide surrogates are added in all the formulations as described above. Vermiculite represents spill control sorbent waste.

Table A-4 gives the surrogate composition with percentages and Table A-5 gives the mixing instructions.

\section{Surrogate Formulation No. 3: Absorbed Aqueous and Organic Liquids}

This formulation, referred to as MWTP-3113/3114, contains about 30 percent combustibles and 50 percent inorganic "MOP-UP or SUP-UP" sorbents (vermiculite and perlite) and 19 percent water. It also contains the RCRA organics, RCRA metal nitrates, and radionuclide surrogates.

The surrogate composition is given in Table A-6 and the mixing instructions are given in Table A-7.

\section{Surrogate Formulation No. 4: High Organic Content Sludge}

This formulation is referred to as MWTP-3122 and includes sludges having hazardous organic materials such as phenolic compounds representing the CPCF sludge waste from Oak Ridge $\mathrm{Y}-12$ plant. It consists of about 50 percent combustibles, inorganic oxides $(\mathrm{Fe}, \mathrm{Ca}, \mathrm{Al}, \mathrm{Si}$ ), RCRA organics, RCRA metal nitrates, and radionuclide surrogates.

The surrogate composition is given in Table A-8 and the mixing instructions are given in Table A-9. 
TABLE A-4:

SURROGATE FORMULATION NO. 2

MWTP 2110 - AQUEOUS HALOGENATED ORGANIC LIQUIDS

\begin{tabular}{|c|c|c|}
\hline No. & COMPONENT NAME & Wt.\% \\
\hline \multicolumn{3}{|c|}{ Bulk Ingredient } \\
\hline $\begin{array}{l}1 \\
2 \\
3 \\
4 \\
5 \\
6 \\
7 \\
8\end{array}$ & $\begin{array}{l}\text { Activated carbon } \\
\text { Cation exchange resin } \\
\text { Water } \\
\text { Toluene } \\
\text { Tetrachloroethylene } \\
\text { Mineral oil } \\
\text { Ethylene glycol } \\
\text { Vermiculite }\end{array}$ & $\begin{array}{r}5 \\
5 \\
10 \\
10 \\
10 \\
10 \\
10 \\
19\end{array}$ \\
\hline \multicolumn{3}{|c|}{ RCRA Metals } \\
\hline $\begin{array}{r}9 \\
10 \\
11 \\
12\end{array}$ & $\begin{array}{l}\mathrm{Cr}\left(\mathrm{NO}_{3}\right)_{2} \bullet 6 \mathrm{H}_{2} \mathrm{O} \\
\mathrm{Ni}\left(\mathrm{NO}_{3}\right)_{2} \bullet 6 \mathrm{H}_{2} \mathrm{O} \\
\mathrm{Pb}\left(\mathrm{NO}_{3}\right)_{2} \\
\mathrm{Cd}\left(\mathrm{NO}_{3}\right)_{2} * 4 \mathrm{H}_{2} \mathrm{O}\end{array}$ & $\begin{array}{l}0.1 \\
0.1 \\
0.1 \\
0.1\end{array}$ \\
\hline \multicolumn{3}{|c|}{ RCRA Organics } \\
\hline $\begin{array}{l}13 \\
14\end{array}$ & $\begin{array}{l}\text { Naphthalene }\left(\mathrm{C}_{10} \mathrm{H}_{8}\right) \\
\text { 1,2-Dichlorobenzene }\left(\mathrm{C}_{6} \mathrm{H}_{4} \mathrm{Cl}_{2}\right)\end{array}$ & $\begin{array}{l}10 \\
10\end{array}$ \\
\hline \multicolumn{3}{|c|}{ Radionuclides } \\
\hline $\begin{array}{l}15 \\
16\end{array}$ & $\begin{array}{l}\mathrm{CeCl}_{3} \\
\mathrm{CsCl}\end{array}$ & $\begin{array}{l}0.3 \\
0.3\end{array}$ \\
\hline
\end{tabular}


TABLE A-5:

MIXING INSTRUCTIONS FOR FORMULATION NO. 2

MWTP 2100 - AQUEOUS HALOGENATED ORGANIC LIQUIDS

STEPNO. SEQUENCE/INSTRUCTIONS

1. Weigh drum and lid and record.

2. Add $100 \%$ of constituents \#4 through \#8 to drum.

3. Stir drum for $\geq 5$ minutes.

4. Weigh drum, lid and mixture and record.

5. In any sequence, carefully add all of each of the 4 RCRA metal compounds (\#912 ) and 2 rad surrogate salts (\#15-\#16) to a separate container. Add enough of \#3 (water) to dissolve the solids.

6. Add $100 \%$ of the cation exchange resin (\#2) to the container (Step 5) and stir.

7. Add the RCRA/RAD metals/resin slurry prepared in Steps 5-6 to drum. Rinse the container with any excess water (\#3) and add to drum.

8. Carefully add the RCRA organics (\#13-\#14) to a separate container. Mix the two components until they are equally intermixed.

9. Slowly add, with constant stirring, the activated carbon (\#1) into the container holding the RCRA organic (\#13-\#14) mixture. Add enough of the activate carbon to absorb the organic liquids.

10. Add the remaining activated carbon not used in Step 9 and the mixture from the container (Steps 7-8) into the drum. Seal the drum.

11. Activate drum mixer for $\geq 15$ minutes. Seal the drum.

12. Weigh drum, lid and surrogate waste stream mixture and record.

13. Measure drum fill level and calculate drum fill capacity. 
TABLE A-6:

SURROGATE FORMULATION NO.3

MWTP 3113/3114 - ABSORBED AQUEOUS AND ORGANIC LIQUIDS

\begin{tabular}{|c|c|c|}
\hline No. & COMPONENT NAME & $\boldsymbol{W t} \%$ \\
\hline Bulk Ingredient & . & \\
\hline 1 & Activated carbon & 5 \\
\hline 2 & Cation exchange resin & 5 \\
\hline 3 & Water & 19 \\
\hline 4 & Perlite & 25 \\
\hline 5 & Vermiculite & 25 \\
\hline 6 & Ethylene glycol & 10 \\
\hline \multicolumn{3}{|l|}{ RCRA Metals } \\
\hline 7 & $\mathrm{Cr}\left(\mathrm{NO}_{3}\right)_{2} \cdot 6 \mathrm{H}_{2} \mathrm{O}$ & 0.1 \\
\hline 8 & $\mathrm{Ni}\left(\mathrm{NO}_{3}\right)_{2} \bullet 6 \mathrm{H}_{2} \mathrm{O}$ & 0.1 \\
\hline 9 & $\mathrm{~Pb}\left(\mathrm{NO}_{3}\right)_{2}$ & 0.1 \\
\hline 10 & $\mathrm{Cd}\left(\mathrm{NO}_{3}\right)_{2} * 4 \mathrm{H}_{2} \mathrm{O}$ & 0.1 \\
\hline \multicolumn{3}{|l|}{ RCRA Organics } \\
\hline 11 & Naphthalene $\left(\mathrm{C}_{10} \mathrm{H}_{8}\right)$ & 5 \\
\hline 12 & 1,2-Dichlorobenzene $\left(\mathrm{C}_{6} \mathrm{H}_{4} \mathrm{Cl}_{2}\right)$ & 5 \\
\hline \multicolumn{3}{|l|}{ Radionuclides } \\
\hline 13 & $\mathrm{CeCl}_{3}$ & 0.3 \\
\hline 14 & $\mathrm{CsCl}$ & 0.3 \\
\hline
\end{tabular}




\section{TABLE A-7:}

\section{MIXING INSTRUCTIONS FOR FORMULATION NO. 3}

MWTP 3113/3114 - ABSORBED AQUEOUS AND ORGANIC LIQUIDS

1. Weigh drum and lid and record.

2. Add $100 \%$ of constituents \#1, \#4 and \#5 (simultaneously or layered) to drum to ensure some mixing of the components.

3. Add components \#6, \#11 and \#12 to the drum.

4. Activate drum mixer for $\geq 5$ minutes.

5. Weigh drum, lid and mixture and record.

6. In any sequence, carefully add all of each of the 4 RCRA metal compounds (\#710 ) and 2 rad surrogate salts (\#13-\#14) to a separate container. Add enough of \#3 (water) to dissolve the solids.

7. Add $100 \%$ of the cation exchange resin (\#2) to the container (Step 6) and stir.

8. Add the RCRA/RAD metals/resin slurry prepared in Steps 6-7 to drum. Rinse the container with any excess water (\#3) and add to drum.

9. Activate drum mixer for $\geq 15$ minutes.

10. Weigh drum, lid and surrogate waste stream mixture and record.

11. Measure drum fill level and calculate drum fill capacity. 
TABLE A-8:

SURROGATE FORMULATION NO.4

\section{MWTP 3122 - HIGH ORGANIC CONTENT SLUDGES}

\begin{tabular}{|c|c|c|}
\hline No. & COMPONENT NAME & $w t \%$ \\
\hline \multicolumn{3}{|c|}{ Bulk Ingredient } \\
\hline 1 & Activated carbon & 10 \\
\hline 2 & Cation exchange resin & 5 \\
\hline 3 & Water & 15 \\
\hline 4 & Perlite & 10 \\
\hline 5 & $\mathrm{Fe}_{2} \mathrm{O}_{3}$ & 10 \\
\hline 6 & $\mathrm{CaSO}_{4} * 2 \mathrm{H}_{2} \mathrm{O}$ & 10 \\
\hline 7 & $\mathrm{Al}_{2} \mathrm{O}_{3}$ & 5 \\
\hline 8 & Ethylene glycol & 10 \\
\hline 9 & Phenol & 10 \\
\hline 10 & Mineral oil & 7 \\
\hline \multicolumn{3}{|c|}{ RCRA Metals } \\
\hline 11 & $\mathrm{Cr}\left(\mathrm{NO}_{3}\right)_{2} \bullet 6 \mathrm{H}_{2} \mathrm{O}$ & 0.1 \\
\hline 12 & $\mathrm{Ni}\left(\mathrm{NO}_{3}\right)_{2} \bullet 6 \mathrm{H}_{2} \mathrm{O}$ & 0.1 \\
\hline 13 & $\mathrm{~Pb}\left(\mathrm{NO}_{3}\right)_{2}$ & 0.1 \\
\hline 14 & $\mathrm{Cd}\left(\mathrm{NO}_{3}\right)_{2} * 4 \mathrm{H}_{2} \mathrm{O}$ & 0.1 \\
\hline \multicolumn{3}{|c|}{ RCRA Organics } \\
\hline 15 & Naphthalene $\left(\mathrm{C}_{10} \mathrm{H}_{8}\right)$ & 2 \\
\hline 16 & 1,2-Dichlorobenzene $\left(\mathrm{C}_{6} \mathrm{H}_{4} \mathrm{Cl}_{2}\right)$ & 5 \\
\hline \multicolumn{3}{|c|}{ Radionuclides } \\
\hline 17 & $\mathrm{CeCl}_{3}$ & 0.3 \\
\hline 18 & $\mathrm{CsCl}$ & 0.3 \\
\hline
\end{tabular}


TABLE A-9:

MIXING INSTRUCTIONS FOR FORMULATION NO. 4

\section{MWTP 3122 - HIGH ORGANIC CONTENT SLUDGES}

STEP NO.

SEQUENCE / INSTRUCTIONS

1. Weigh drum and lid and record.

2. In order, add $100 \%$ of constituents \#1, \#4 and \#5 through \#10, \#15 and \#16 to drum.

3. Activate drum mixer for $\geq 5$ minutes.

4. Weigh drum, lid and mixture and record.

5. In any sequence, carefully add all of each of the 4 RCRA metal compounds (\#11$\# 14$ ) and 2 rad surrogate salts (\#17-\#18) to a separate container. Add enough of \#3 (water) to dissolve the solids.

6. Add $100 \%$ of the cation exchange resin (\#2) to the container (Step 5) and stir.

7. Add the RCRA/RAD metals/resin slurry prepared in Steps 5-6 to drum. Rinse the container with any excess water (\#3) and add to drum.

8. Activate drum mixer for $\geq 15$ minutes.

9. Weigh drum, lid and surrogate waste stream mixture and record.

10. Measure drum fill level and calculate drum fill capacity. 
Surrogate Formulation No. 5: Cement Sludge, Ash, and Solids

This formulation, referred to as MWTP-3140, contains about 16 percent combustibles and 53 percents inorganics (cement or concrete, solidifying agents, plaster of paris, fly ash, alumina, and perlite) and 30 percent water. It also contains the RCRA organics, RCRA metal nitrates, and radionuclide surrogates.

The surrogate composition is given in Table A-10 and the mixing instructions are given in Table A-11.

\section{Surrogate Formulation No. 6: Natural Aqueous Waste}

This formulation is referred to as MWTP- 1300 and is an aqueous waste stream with water up to 75 percent and a $\mathrm{pH}$ value ranging from 2 to 12.5 (acidic, neutral or basic). It may contain phosphates, nitrates, chlorides and sulfates of inorganic metals $(\mathrm{Ca}, \mathrm{Al}, \mathrm{Na}, \mathrm{Mg}), \mathrm{RCRA}$ organics, RCRA metal nitrates, and radionuclide surrogates. It also simulates well the supernatant tank wastes when the magnesium sulfate is substituted with aluminum nitrate and the chlorides of cesium and cerium are substituted with their nitrates.

The surrogate composition is given in Table A-12 and the mixing instructions are given in Table A-13. 
TABLE A-10:

SURROGATE FORMULATION NO. 5

\section{MWTP 3140 - CEMENT SLUDGE, ASH AND SOLIDS}

\begin{tabular}{|c|c|c|}
\hline No. & COMPONENT NAME & $W_{2} \%$ \\
\hline \multicolumn{3}{|c|}{ Bulk Ingredient } \\
\hline 1 & Activated carbon & 10 \\
\hline 2 & Cation exchange resin & 5 \\
\hline 3 & Water & 30 \\
\hline 4 & Perlite & 10 \\
\hline 5 & Fly ash (ASTM Class F) & 15 \\
\hline 6 & Concrete (cured, crushed, screened) & 20 \\
\hline 7 & $\mathrm{CaSO}_{4}{ }^{*} 0.5 \mathrm{H}_{2} \mathrm{O}$ (plaster of paris) & 3 \\
\hline 8 & $\mathrm{Fe}_{2} \mathrm{O}_{3}$ (Hematite) & 3 \\
\hline 9 & $\mathrm{Al}_{2} \mathrm{O}_{3}$ & 2 \\
\hline \multicolumn{3}{|c|}{ RCRA Metals } \\
\hline 10 & $\mathrm{Cr}\left(\mathrm{NO}_{3}\right)_{2} \bullet 6 \mathrm{H}_{2} \mathrm{O}$ & 0.1 \\
\hline 11 & $\mathrm{Ni}\left(\mathrm{NO}_{3}\right)_{2} \bullet 6 \mathrm{H}_{2} \mathrm{O}$ & 0.1 \\
\hline 12 & $\mathrm{~Pb}\left(\mathrm{NO}_{3}\right)_{2}$ & 0.1 \\
\hline 13 & $\mathrm{Cd}\left(\mathrm{NO}_{3}\right)_{2} * 4 \mathrm{H}_{2} \mathrm{O}$ & 0.1 \\
\hline \multicolumn{3}{|c|}{ RCRA Organics } \\
\hline 14 & Naphthalene $\left(\mathrm{C}_{10} \mathrm{H}_{8}\right)$ & 0.5 \\
\hline 15 & 1,2-Dichlorobenzene $\left(\mathrm{C}_{6} \mathrm{H}_{4} \mathrm{Cl}_{2}\right)$ & 0.5 \\
\hline \multicolumn{3}{|c|}{ Radionuclides } \\
\hline 16 & $\mathrm{CeCl}_{3}$ & 0.3 \\
\hline 17 & $\mathrm{CsCl}$ & 0.3 \\
\hline
\end{tabular}




\section{MIXING INSTRUCTIONS FOR FORMULATION NO. 5}

\section{MWTP 3140 - CEMENT SLUDGE, ASH AND SOLIDS}

STEP NO.

SEQUENCE / INSTRUCTIONS

1. Weigh drum and lid and record.

2. In sequential order, add $100 \%$ of constituents \#4 through \#9 to drum.

3. Activate drum mixer for $\geq 5$ minutes.

4. Weigh drum, lid and mixture and record.

5. In any sequence, carefully add all of each of the 4 RCRA metal compounds (\#10$\# 13$ ) and 2 rad surrogate salts (\#16-\#17) to a separate container. Add enough of \#3 (water) to dissolve.

6. Add $100 \%$ of the cation exchange resin (\#2) to the container (Step 5) and stir.

7. Add the RCRA/RAD metals/resin slurry prepared in Steps 5-6 to drum. Rinse the drum with any excess water (\#3) and add to drum.

8. Activate drum mixer for $\geq 5$ minutes.

9. Weigh drum, lid and mixture and record.

10. Carefully add the RCRA organics (\#14-\#15) to a separate container. Mix the two components until they are equally intermixed.

11. Slowly add, with constant stirring, the activated carbon (\#1) into the container holding the RCRA organic (\#14-\#15) mixture. Add enough of the activate carbon to absorb the organic liquids.

12. Add the remaining activated carbon not used in Step 11 and the mixture from the container (Steps 10-11) into the drum. Seal the drum.

13. Activate drum mixer for $\geq 15$ minutes.

14. Weigh drum, lid and surrogate waste stream mixture and record.

15. Measure drum fill level and calculate drum fill capacity. 
TABLE A-12:

SURROGATE FORMULATION NO. 6

MWTP 1300 - NATURAL AQUEOUS WASTE

\begin{tabular}{|c|c|c|}
\hline No. & COMPONENT NAME & $\boldsymbol{W t} \%$ \\
\hline \multicolumn{3}{|c|}{ Bulk Ingredient } \\
\hline 1 & Activated carbon & 5 \\
\hline 2 & Cation exchange resin & 5 \\
\hline 3 & Water & 75 \\
\hline 4 & $\mathrm{CaCl}_{2} 6$ & 3 \\
\hline 5 & $\mathrm{NaNO}_{3}$ & 3 \\
\hline 6 & $\mathrm{Al}\left(\mathrm{NO}_{3}\right)_{3}$ & 3 \\
\hline 7 & $\mathrm{Na}_{2} \mathrm{HPO}_{4} 7 \mathrm{H}_{2} \mathrm{O}$ & 3 \\
\hline 8 & Fly ash (ASTM Class F) & 1 \\
\hline \multicolumn{3}{|c|}{ RCRA Metals } \\
\hline 9 & $\mathrm{Cr}\left(\mathrm{NO}_{3}\right)_{2} \bullet 6 \mathrm{H}_{2} \mathrm{O}$ & 0.1 \\
\hline 10 & $\mathrm{Ni}\left(\mathrm{NO}_{3}\right)_{2} \bullet 6 \mathrm{H}_{2} \mathrm{O}$ & 0.1 \\
\hline 11 & $\mathrm{~Pb}\left(\mathrm{NO}_{3}\right)_{2}$ & 0.1 \\
\hline 12 & $\mathrm{Cd}\left(\mathrm{NO}_{3}\right)_{2} * 4 \mathrm{H}_{2} \mathrm{O}$ & 0.1 \\
\hline \multicolumn{3}{|c|}{ RCRA Organics } \\
\hline 13 & Naphthalene $\left(\mathrm{C}_{10} \mathrm{H}_{8}\right)$ & 0.5 \\
\hline 14 & 1,2-Dichlorobenzene $\left(\mathrm{C}_{6} \mathrm{H}_{4} \mathrm{Cl}_{2}\right)$ & 0.5 \\
\hline \multicolumn{3}{|c|}{ Radionuclides } \\
\hline 15 & $\mathrm{CeCl}_{3}$ & 0.3 \\
\hline 16 & $\mathrm{CsCl}$ & 0.3 \\
\hline
\end{tabular}




\section{MWTP 1300 - NATURAL AQUEOUS WASTE}

1. Weigh drum and lid and record.

2. Add $3 / 4$ of \#3 (water) and $100 \%$ of constituents \#4 through \#8 to drum.

3. Activate drum mixer for $\geq 5$ minutes.

4. Weigh drum, lid and mixture and record.

5. In any sequence, carefully add all of each of the 4 RCRA metal compounds (\#9$\# 12$ ) and 2 rad surrogate salts (\#15-\#16) to a separate container. Add enough of \#3 (water) to dissolve.

6. Add $100 \%$ of the cation exchange resin (\#2) to the container (Step 5) and stir.

7. Add the RCRA/RAD metals/resin slurry prepared in Steps 5-6 to drum. Rinse the drum with any excess water (\#3) and add to drum.

8. Carefully add the RCRA organics (\#13-\#14) to a separate container. Mix the two components until they are equally intermixed.

9. Slowly add, with constant stirring, the activated carbon (\#1) into the container holding the RCRA organic (\#13-\#14) mixture. Add enough of the activated carbon to absorb the organic liquids.

10. Add the remaining activated carbon not used in Step 9 and the mixture from the container (Steps 7-8) into the drum. Seal the drum.

11. Activate drum mixer for $\geq 15$ minutes. Seal the drum.

12. Weigh drum, lid and surrogate waste stream mixture and record.

13. Measure drum fill level and calculate drum fill capacity. 


\section{Surrogate Formulation No. 7: Piketon Waste}

Through site visits and inquiries, ThermoChem has obtained information on the detailed physical, chemical, and radioactive characteristics of PCB- and Uranium-contaminated LLMW located at the Portsmouth Gaseous Diffusion Plant. Based on this data, ThermoChem has formulated a seventh surrogate waste stream for testing in the PDU. The feedstock composition is given in Table A-14. The bulk ingredients are sorbent (polypropylene absorbent pads) and oil, the RCRA organic is monochlorobenzene (surrogate for PCB), and the radionuclide is a mixture of cerium fluoride $\left(\mathrm{CeF}_{3}\right)$ and Cerium oxide $(\mathrm{CeO})$ (surrogates for Uranyl fluoride $\left(\mathrm{UO}_{2} \mathrm{~F}_{2}\right)$, Uranium Penta/tetrafluoride ( $\left(\mathrm{UF}_{5} / \mathrm{UF}_{4}\right)$ and Uranium oxides). Predominantly, it is planned to use the nominal composition. Two tests (HIGH1 and HIGH2) of 8-hour duration each are planned with high concentrations of monochlorobenzene and cerium compounds to very accurately quantify (i) the Destruction and Removal Efficiency (DRE) of monochlorobenzene (to 6 nines or better), and (ii) cerium retention in the system ( 4 nines or better). Three short tests of 8 hours each are also planned (MERC1, MERC2 and MERC3) to investigate mercury retention in the system. The three mercury compounds to be tested are elemental mercury, mercury in reduced form - mercurous chloride, and mercury in oxidized form - mercury oxide.

\section{FEEDSTOCK PREPARATION}

Approximately 500 to 1,500 pounds of feedstock will be made up just before the start of each test. Feedstock preparation will be based on the procedures as indicated in Section 3.2. Preparation of the feedstock will be witnessed, audited, and certified by a staff member from the analytical service group. The heterogeneous nature of the various feedstocks makes it extremely difficult to sample and assay. The volatile nature of some of the organic constituents requires the feedstock to be prepared just before an operational test to assure that the constituents will be present in the actual feed to the steam reformer. The seven feedstocks are as follows:

\section{No. $\quad$ Feedstock}

1) Heterogeneous debris;

2) Aqueous halogenated organic liquids; 


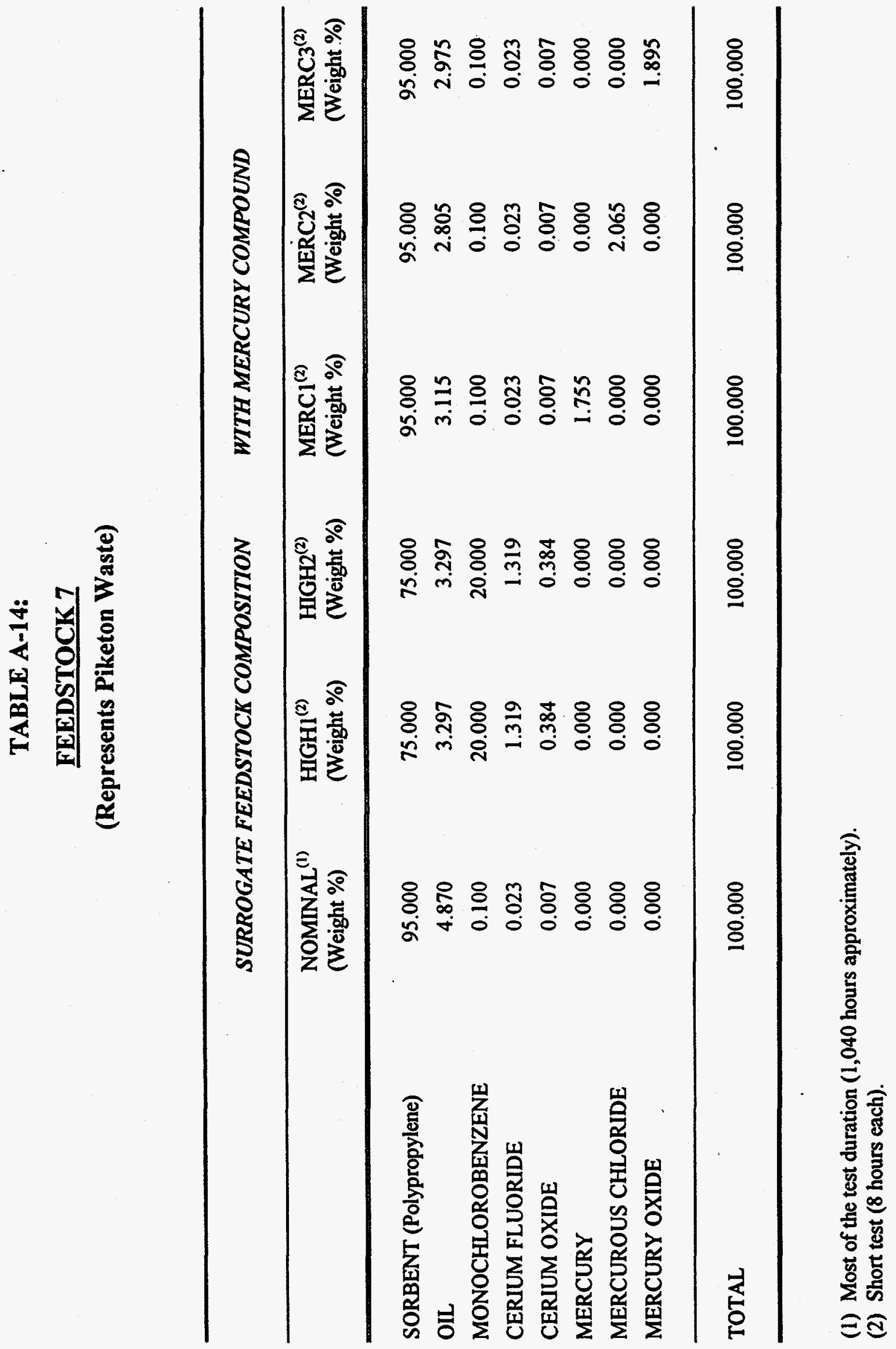


Absorbed aqueous organic liquids;

4) High organic content sludge;

5) Cement, sludge, ash, and solids;

6) Natural aqueous waste; and

Piketon Waste.

Feedstock raw materials will be purchased from known suppliers and kept in unopened or closed containers until the feedstock is to be made up. Some of the organic components may be made up as a stock solution by the analytical service group prior to the start of a test run. The prepared feedstock is to be kept in a closed 55-gallon drum or appropriate container. To the extent possible, the drum is to be kept in a cool area and out of direct sunlight. A log sheet is to be prepared indicating date and time, final weight of the feedstock, weight, source, purity of the components, and the name and signature of the analytical service group representative.

The proposed feedstock testing order is No. 6, No. 2 , No. 4 , No. 1, No. 3 , No. 5 , and No. 7 It is expected that laboratory analysis will be available within three weeks after the samples have been taken. Results of the initial testing will be used to revise conditions, as necessary, for the subsequent tests. The rationale for this order is twofold: Feedstock No. 6 is mostly an aqueous feed material with a minimum amount of solid residue (ash). Feedstock No. 2 is also easy to feed. This feedstock requires the lock-hopper feeder subsystem. The most difficult material to feed is Feedstock No. 5 which contains a high percentage of residual solids that must be removed from the first-stage steam reformer bed on an hourly basis. This selection of feedstock test order allows ThermoChem/MTCI to learn more about the actual hardware and individual equipment items, or process functions, one item or function at a time. Ground glass frit or glass ballotini (150 microns mean size) will be purchased and fed into the first-stage steam reformer along with the feedstock to simulate vitrification operation.

Ion exchange resins are included in each of the six surrogates in small quantities. Ion exchange resins as beads and powders represent a significant waste stream from both DOE and commercial sources. Resins tested separately would provide valuable information on yet another possible waste stream for the steam reformer technology. A screening test will be done on surrogate ion exchange 
resins in a smaller test unit prior to PDU testing to acquire operating experience and collect preliminary data on performance.

For the seventh feedstock, the sorbent pads (polypropylene) will be shredded and stored in 55gallon drums. A mixture of mineral oil and monochlorobenzene in the proportions listed in Table A-14 will be prepared. This mixture will be prepared on a daily basis and stored in 5-gallon buckets (for nominal composition) or 30-gallon drums (for HIGHl and HIGH2 compositions). The shredded pads will be metered through the screw feeder while the liquid mixture will be pumped and sprayed on to the shredded material via an injection port in the injection screw. The cerium compounds (cerium fluoride and cerium oxide) are immiscible with oil-monochlorobenzene mixture and also are insoluble in water. Therefore, additional investigation is needed to prepare a uniformly blended feedstock. We plan to perform some bench-scale tests with the feedstock constituents to identify the most appropriate feed method for each of the components commensurate with uniformity of feed composition. We also propose to contact Sandia National Laboratory and Idaho National Engineering Laboratory staff to get their input in this matter.

The mercury compounds pose a feeding difficulty similar to that of the cerium compounds. Additionally, they are extremely toxic and require stringent health and safety procedures in handling. Therefore, it is proposed to conduct additional investigations as in the case of cerium compounds to identify the appropriate procedures for feedstock preparation and injection into the steam reformer. 


\section{APPENDIX B:}

\section{TASK 1.2.1: REVISED TEST PLAN}

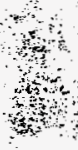




\title{
TASK 1.2.1: REVISED TEST PLAN
}

\author{
Prepared for:
}

Steam Reforming of Low-Level Mixed Waste

Department of Energy. Federal Energy Technology Center Morgantown, West Virginia

Under:

Contract No. DE-AR21-95MC32091

Prepared by:

ThermoChem, Inc.

March 1996

Revised as per modification A009: June 1997 


\section{TABLE OF CONTENTS}

Page

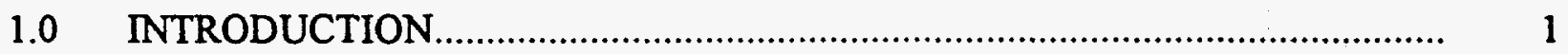

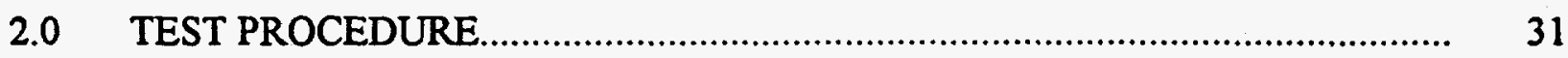

$2.1 \quad$ FEEDSTOCK PREPARATION......................................................... 31

$2.2 \quad$ START-UP AND OPERATIONAL PROCEDURES ................................ 33

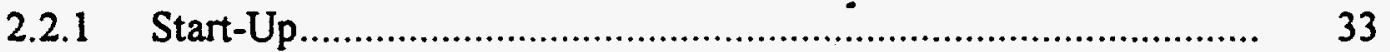

2.2.2 Feed Subsystem ............................................................. 34

2.2.3 Test Matrix ........................................................................ 35

2.2.4 Normal Shutdown Procedures.................................................. 43

3.0 EMERGENCY SHUTDOWN ....................................................................... 45

3.1 OVER-PRESSURE EMERGENCY SHUTDOWN …….......................... 45

3.2 OVER-TEMPERATURE EMERGENCY SHUTDOWN........................... 46

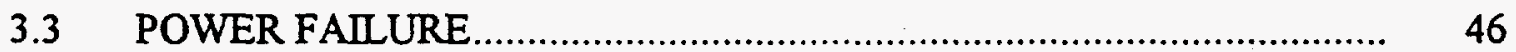

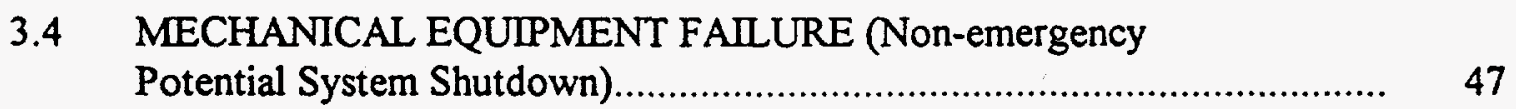

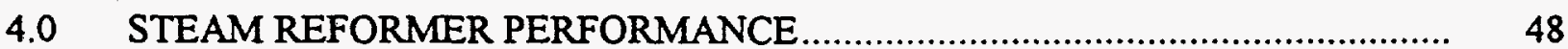

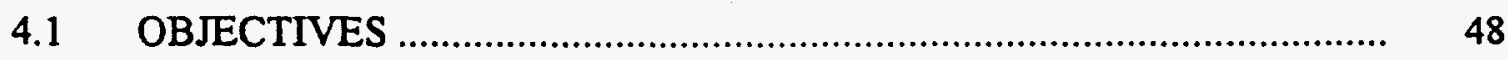

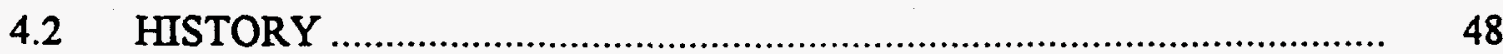

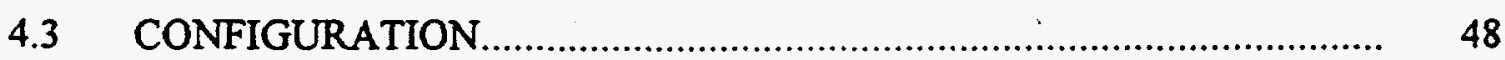

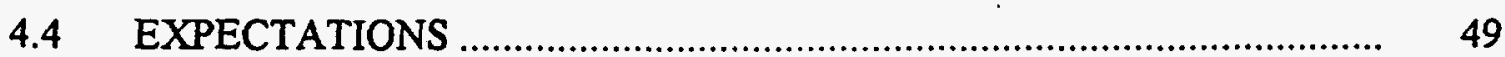




\section{TABLE OF CONTENTS}

(Cont'd)

Page

5.0 CALIBRATION ............................................................................. 50

5.1 LOCK-HOPPER FEEDER SUBSYSTEM.............................................. 50

5.2 LIQUID FEED SYSTEM ................................................................ 50

5.3 TEMPERATURE ELEMENT CALIBRATION ………………………….... 51

5.4 PRESSURE INDICATORS .............................................................. 51

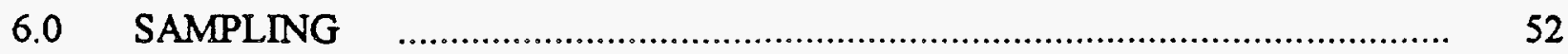

6.1 GAS SAMPLING ......................................................................... 52

6.2 SOLIDS SAMPLING .................................................................... 53

7.0 IDENTIFICATION, ASSESSMENT, AND QUANTIFICATION

OF SOURCES OF ERROR .......................................................................

7.1 BED MATERIAL AND SOLID RESIDUE …………………………….... 54

7.2 CHLORINE BALANCE ..................................................................... 54

7.3 ORGANICS AND RCRA ORGANIC ………………………………...... 55

8.0 RESIDUAL WASTE DISPOSAL ………………………………………….... 57

$9.0 \quad$ VITRIFICATION TESTS .......................................................................... 58 


\section{LIST OF FIGURES}

$\underline{\text { Page }}$

FIGURE 1 PROCESS FLOW DIAGRAM OF SURROGATE

WASTE TREATMENT.

2

FIGURE 2 PROCESS FLOW DIAGRAM FOR WASTE TREATMENT

SIMULATION IN THE PDU .............................................................. 18

FIGURE 3 DIAGRAM OF INSTRUMENTATION FOR SURROGATE TREATMENT 


\section{LIST OF TABLES}

Page

TABLE 1 SURROGATE FORMULATION - SUMMARY …………..................... 5

TABLE 2 ULTIMATE ANALYSIS OF THE SURROGATE FEEDSTOCKS ............ 7

TABLE $3 \quad$ FEEDSTOCK NO. 7 (REPRESENTS PIKETON WASTE) ……............... 9

TABLE 4 PRELIMINARY DESIGN SPECIFICATIONS FOR THE SURROGATE WASTE TREATMENT PDU ........................................ 10

TABLE 5 SURROGATE WASTE THERMAL TREATMENT

SIMULATION SUMMARY (Feedstock No. 1) …….................................. 12

TABLE 6 SURROGATE WASTE THERMAL TREATMENT SIMULATION SUMMARY (Feedstock No. 2) ........................................ 13

TABLE 7 SURROGATE WASTE THERMAL TREATMENT

SIMULATION SUMMARY (Feedstock No. 3)........................................ 14

TABLE 8 SURROGATE WASTE THERMAL TREATMENT SIMULATION SUMMARY (Feedstock No. 4)........................................ 15

TABLE 9 SURROGATE WASTE THERMAL TREATMENT SIMULATION SUMMARY (Feedstock No. 5)....................................... 16

TABLE 10 SURROGATE WASTE THERMAL TREATMENT SIMULATION SUMMARY (Feedstock No. 6),.......................................... 17

TABLE 11 PROCESS FLOW DATA SHEET FOR WASTE SIMULATION IN THE PDU - FEEDSTOCK 7 .

TABLE 12 FEEDSTOCK 7 WASTE THERMAL TREATMENT SIMULATION SUMMARY.......................................................... 21

TABLE 13 POHC CONCENTRATION IN FLUE GAS (Feedstock No. 1) .................. 23 


\section{LIST OF TABLES}

(Cont'd)

Page

TABLE 14 POHC CONCENTRATION IN FLUE GAS (Feedstock No. 2) .................. 24

TABLE 15 POHC CONCENTRATION IN FLUE GAS (Feedstock No. 3) .................. 25

TABLE 16 POHC CONCENTRATION IN FLUE GAS (Feedstock No. 4) ................. 26

TABLE 17 POHC CONCENTRATION IN FLUE GAS (Feedstock No. 5) ................. 27

TABLE 18 POHC CONCENTRATION IN FLUE GAS (Feedstock No. 6) ................. 28

TABLE 19 DETECTION LIMITS INDICATED BY RADIAN CORPORATION ...... 29

TABLE 20 TEST MATRIX OF SURROGATE TREATMENT .................................. 36

TABLE 21 TEST MATRIX - SEVENTH FEEDSTOCK ………………………….... 38 


\section{STEAM REFORMING OF LOW-LEVEL MIXED WASTE \\ Contract Number DE-AR21-95MC32091}

\section{TASK 1.2.1: REVISED TEST PLAN}

SECTION 1.0

INTRODUCTION

In accordance with the Modified Statement of Work provided by Modification $A 009$ of Contract No. DE-AR21-95MC32091, Steam Reforming of Low-Level Mixed Waste, the Task 1.2.1, Revised Test Plan is revised once again. This Revised Test Plan has been developed to ensure accomplishment of the overall obligations of the contract in a timely and cost-effective manner. This Revised Test Plan includes a detailed description of all tests to be performed, including testing conditions, test duration, parameters to be verifed, and performance goals and success criteria.

The process developed for surrogate low-level mixed waste (LLMW) treatment (Figure 1) consists of:

- $\quad$ First-stage steam reformer

- Second-stage steam reformer

- Flameless Thermal Oxidizer and Cleanup Containing:

- Spray cooler/scrubber

- Soda ash water solution feed system for the scrubber

- HEPA filter

- Activated carbon filter

- I.D. fan

- $\quad$ Stack

- Boiler

- Steam superheater 


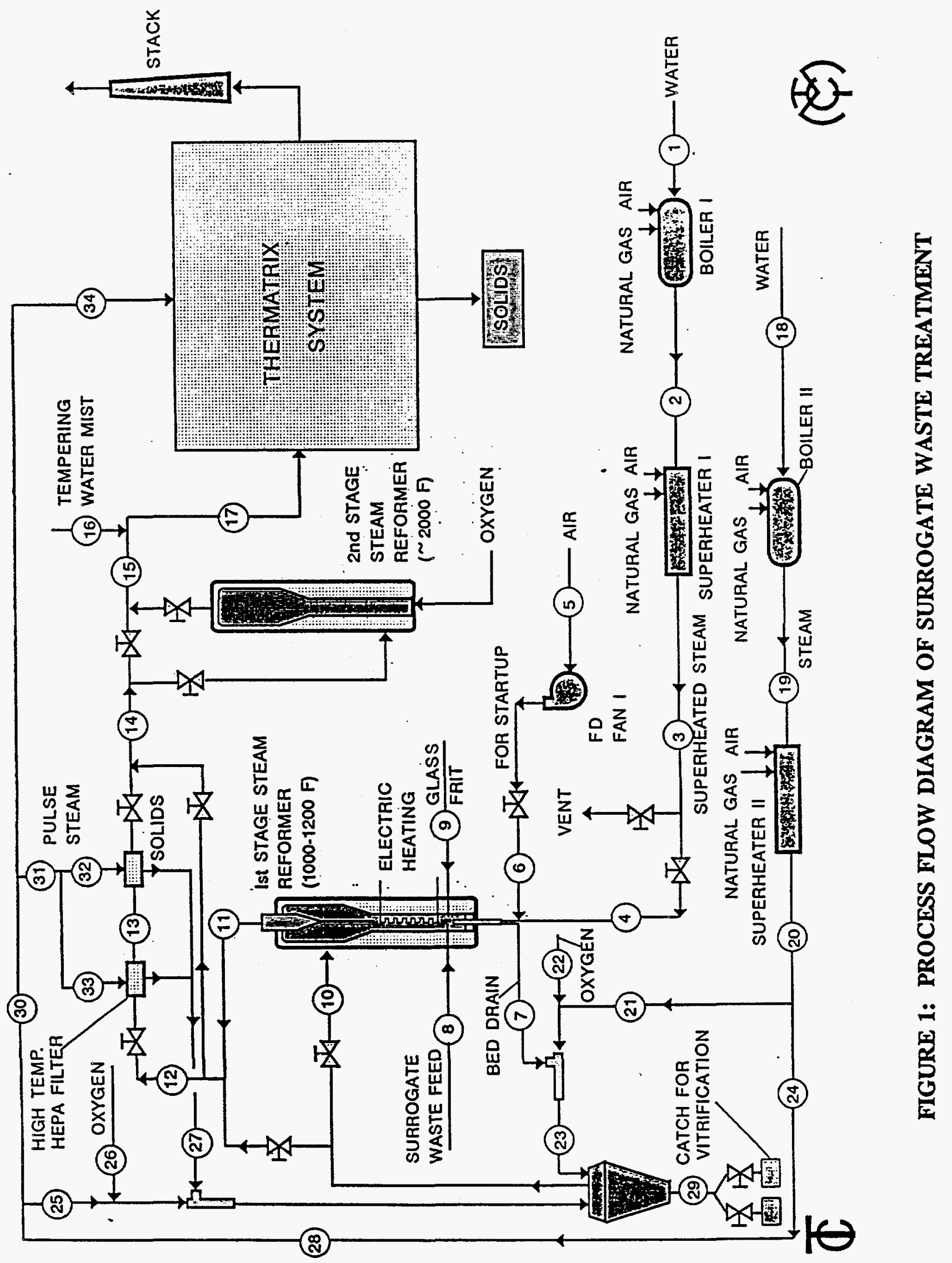


The system consists of three basic steps, viz. steam reforming, flameless thermal oxidation and vitrification. The surrogate waste feed is fed into the first-steam reformer through a Moyno pump subsystem if it's a liquor or a screw feeder subsystem if it's a solid. Glass frit of 150 to 250 microns mean size is also fed into the first-stage steam reformer at a rate commensurate with the composition and amount of inorganics present in the waste. The reformer is refractory-lined in view of the presence of both sulfur and chlorine in the waste feed. The reformer is electrically heated by heating rods immersed in the dense fluidized bed. The bed material could either be glass fit or sand. The sand/frit makeup will be optimized to produce the best vitrified final waste. The bed is fluidized by a combination of superheated steam and natural gas at a ratio determined by the organic content of the waste stream. The steam is generated in a boiler and superheated to near bed temperature to minimize the load on the electrical heater. With the use of superheated steam alone, the superficial fluidization velocity turned out to be low $(<1 \mathrm{f} / \mathrm{s})$ for normal steam-to-carbon-mass ratios ( 2 to 4.5$)$ for some of the surrogate mixtures. A higher fluidization velocity is required $(>1.5 \mathrm{ft} / \mathrm{s})$ to ensure uniform fluidization and to prevent hot spots. Natural gas is a natural choice for it not only supplements fluidization, but also forms a fuel source to achieve $\sim 2000^{\circ} \mathrm{F}$ in the second-stage steam reformer and the thermal oxidizer. Depending on the feedstock, the natural gas-superheated steam mixture ratio is varied to maintain flammability and achieve $\sim 2000^{\circ} \mathrm{F}$ temperature in the flameless thermal oxidizer. The first-stage steam reformer temperature is kept low $\left(1000\right.$ to $\left.1100^{\circ} \mathrm{F}\right)$ to prevent the carryover of radionuclides in the upflow stream and maximize the retention of these species in the bed and subsequent flow into the catch for vitrification. The electric heat provides the energy for sensible and latent heating of the feedstock and for the endothermic heat of reaction. For a given heat input, the lower the bed temperature the higher the waste throughput. A high-temperature pulse jet HEPA filter at the exit of the first-stage steam reformer serves to minimize the carryover of particulates in the product gas stream. Due to low-temperature operation, the natural gas is not expected to crack or reform but just flow through. The bed drain rate is regulated to maintain a constant bed depth.

The second-stage steam reformer is operated in the autothermal and plug flow mode. Again, this unit is refractory-lined. By supplying oxygen rather than air to minimize volumetric flow and in turn the size of the unit, the exothermic reactions are favored partially to supply the energy required to raise the temperature and promote additional steam-reforming reactions. The objectives of the second 
stage is to further break the bonds and the halogen rings/compounds and enhance the formation of $\mathrm{HCl}$ and $\mathrm{H}_{2} \mathrm{~S} / \mathrm{SO}_{2}$. Note that the reaction environment is substoichiometric and hydrogen-rich. The second stage is to be configured with a valving arrangement such that the overall system can be operated with the second stage either on-line or off-line. The product gases flow into a flameless thermal oxidizer supplied by Thermatrix. Air is added here to achieve superstoichiometry ( $>100 \%$ excess air) and burn-out of the product gases. The Thermatrix unit is being warranted to provide $99.99 \%$ destruction efficiency and $99.9999 \%$ in the case of PCBs.

The flue gases then flow into a hot gas cleanup subsystem also supplied by Thermatrix. The system is intended to consist of a spray cooler/scrubber, HEPA filter, and activated carbon filters. Water is added to quench the flue gas stream while soda ash is added to scrub $\mathrm{HCl}$ and $\mathrm{SO}_{2}$. The HEPA filter inlet temperature is regulated to about $250^{\circ} \mathrm{F}$. Compressed air is used to back-flush the filter and keep it clean. The spent sorbent is collected dry in the filter catch. An activated carbon filter is used to capture any remaining metal vapors and radionuclide species. In a commercial system, the spent carbon can be processed in the steam reformer. An induced draft fan is used to maintain negative or suction pressure throughout the entire system. The balance or zero pressure point would be the freeboard of the first-stage steam reformer.

The surrogate feedstock list from the surrogate feedstock identification report (Task 1.1) is presented in Table 1. The components of the system from the first-stage steam reformer to the inlet of the spray cooler are totally refractory-lined in view of the simultaneously high chlorine and sulfur content of the feedstocks (see Table 2).

A survey of LLMW at DOE sites has resulted in the identification of a large inventory of PolyChlorinated BiPhenyl (PCB) solid waste at the Portsmouth Gaseous Diffusion Plant as a desirable feed for further tests. About 20 to 25 percent of the DOE LLMW inventory includes halogenated organics such as PCB. The PCB-contaminated waste at Portsmouth is now contained in approximately 15,000 barrels and amounts to about 3 million pounds. This waste was previously plamed for destruction at the TSCA incinerator but is not now scheduled for processing until well into the 21st century. No other options appear to be available or planned 
TABLE 1:

\section{SURROGATE FORMULATION - SUMMARY}

\begin{tabular}{|c|c|c|c|c|c|c|}
\hline 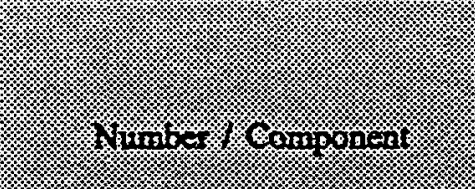 & 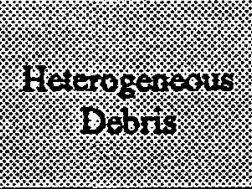 & 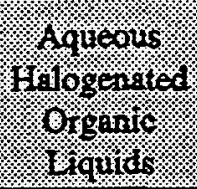 & 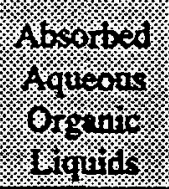 & 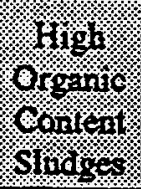 & 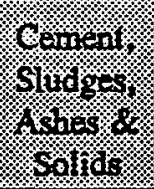 & 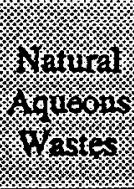 \\
\hline & $x$ & 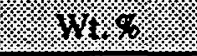 & 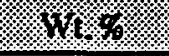 & $14 \%$ & W/. & Ww. \\
\hline $\begin{array}{l}\text { Bulk Ingredient } \\
\text { 1. Activated Carbon } \\
\end{array}$ & 5. & 5. & 5. & 10. & 10. & 5. \\
\hline 2. Cation Exchange Resin & 5. & 5. & 5. & 5. & 5. & 5. \\
\hline 3. Water & 10. & 10. & 19. & 15. & 30. & 75. \\
\hline 4. Wood & 10. & & & & & \\
\hline 5. Polyvinylchloride (PVC) & 10. & & & & & \\
\hline 6. Neoprene & 10. & & & & & \\
\hline 7. Mild Steel/Hematite/ $\mathrm{Fe}_{2} \mathrm{O}_{3}$ & 10. & & & 10. & 3. & \\
\hline 8. Glass Beads & 10. & & & & & \\
\hline 9. Cement/Concrete & 8. & & & & & \\
\hline 10. Alumina/ $\mathrm{Al}_{2} \mathrm{O}_{3}$ & 10. & & & 5. & 2. & \\
\hline 11. Diatomaceous Earth & 10. & & & & & \\
\hline 12. Toluene & & 10. & & & & \\
\hline 13. Tetrachloroethylene & & 10. & & & & \\
\hline 14. Mineral Oil & & 10. & & 7. & & \\
\hline 15. Ethylene Glycol & & 10. & 10. & 10. & & \\
\hline 16. Vermiculite & & 19. & 25. & & & \\
\hline 17. Perlite $\left(\mathrm{SiO}_{4}\right)$ & & & 25. & 10. & 10. & \\
\hline $\begin{array}{l}\text { 18. } \mathrm{CaSO}_{4} \cdot 2 \mathrm{H}_{2} \mathrm{O} / \mathrm{Plaster} \text { of } \\
\text { Paris }\end{array}$ & & & & 10. & 3. & \\
\hline 19. Phenol & & & & 10. & & \\
\hline 20. Fly Ash (ASTM Class F) & & & & & 15. & 1. \\
\hline $\begin{array}{l}\text { 21. Concrete (cured, crushed, } \\
\text { screened) }\end{array}$ & & & & & 20. & \\
\hline
\end{tabular}




\section{TABLE 1:}

SURROGATE FORMULATION - SUMMARY

(Cont'd)

\begin{tabular}{|c|c|c|c|c|c|c|}
\hline 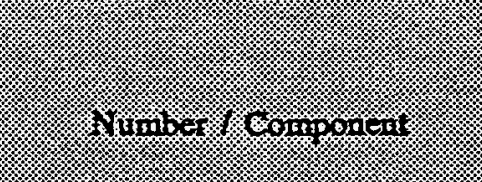 & 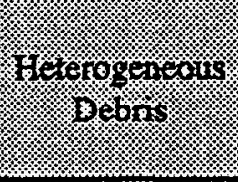 & Holonger & 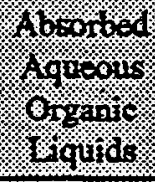 & 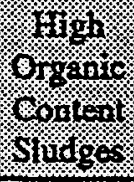 & 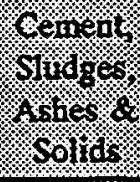 & 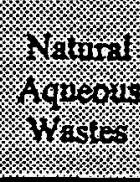 \\
\hline & $w$ & $8 \%$ & wis. & 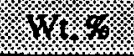 & W\% & w. \\
\hline 22. $\mathrm{CaCl}_{2}$ & & & & & & 3. \\
\hline 23. $\mathrm{NaHCO}_{3} / \mathrm{NaNO}_{3} \bullet$ & . & & 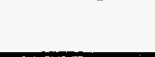 & & & 3. \\
\hline 24. $\mathrm{MgSO}_{4} \bullet 7 \mathrm{H}_{2} \mathrm{O} / \mathrm{Al}\left(\mathrm{NO}_{3}\right)_{3}$ & & & & & & 3. \\
\hline 25. $\mathrm{Na}_{2} \mathrm{HPO}_{4} \bullet 7 \mathrm{H}_{2} \mathrm{O}$ & & & & & & 3. \\
\hline $\begin{array}{l}\text { RCRA Metals } \\
\text { 26. } \mathrm{Cr}\left(\mathrm{NO}_{3}\right)_{2} \cdot 6 \mathrm{H}_{2} \mathrm{O} \text { (or oxide) } \\
\end{array}$ & 0.1 & 0.1 & 0.1 & 0.1 & 0.1 & 0.1 \\
\hline 27. $\mathrm{Ni}\left(\mathrm{NO}_{3}\right)_{2} \cdot 6 \mathrm{H}_{2} \mathrm{O}$ & 0.1 & 0.1 & 0.1 & 0.1 & 0.1 & 0.1 \\
\hline 28. $\mathrm{Pb}\left(\mathrm{NO}_{3}\right)_{2}$ & 0.1 & 0.1 & 0.1 & 0.1 & 0.1 & 0.1 \\
\hline 29. $\mathrm{Cd}\left(\mathrm{NO}_{3}\right)_{2} \bullet 4 \mathrm{H}_{2} \mathrm{O}$. & 0.1 & 0.1 & 0.1 & 0.1 & 0.1 & 0.1 \\
\hline $\begin{array}{l}\text { RCRA Organics } \\
\text { 30. Naphthalene }\left(\mathrm{C}_{10} \mathrm{H}_{8}\right) \\
\end{array}$ & 0.5 & 10. & 5. & 2. & 0.5 & 0.5 \\
\hline $\begin{array}{l}\text { 31. 1,2-Dichlorobenzene } \\
\left(\mathrm{C}_{6} \mathrm{H}_{4} \mathrm{Cl}_{2}\right)\end{array}$ & 0.5 & 10. & 5. & 5. & 0.5 & 0.5 \\
\hline $\begin{array}{l}\text { Radionuclide Surrogate } \\
\text { 32. } \mathrm{CeCl}_{3}\end{array}$ & 0.3 & 0.3 & 0.3 & 0.3 & 0.3 & 0.3 \\
\hline 33. $\mathrm{CsCl} / \mathrm{CsNO}_{3}$ & 0.3 & 0.3 & 0.3 & 0.3 & 0.3 & 0.3 \\
\hline
\end{tabular}

* Suggested substitute for surrogate Feedstock No. 6 only. 
TABLE 2:

ULTIMATE ANALYSIS OF THE SURROGATE FEEDSTOCKS

\begin{tabular}{|c|c|c|c|c|c|c|c|}
\hline \multirow[b]{3}{*}{ COMPOSITION } & \multicolumn{7}{|c|}{ FEEDSTOCK (Wt. \%) } \\
\hline & 1 & 2 & 3 & 4 & 5 & 6 & 7 \\
\hline & $N \quad H D$ & AHOL & AAOL & HOCS & - CSAS & NAW & $P W$ \\
\hline Ash & 50.27 & 19.57 & 50.57 & 28.83 & 51.55 & 6.55 & 0.03 \\
\hline Moisture & 13.36 & 12.56 & 21.56 & 19.65 & 33.19 & 79.30 & 0.00 \\
\hline Carbon & 20.72 & 44.06 & 17.75 & 33.61 & 12.45 & 7.67 & 85.64 \\
\hline Hydrogen & 1.77 & 4.32 & 1.60 & 3.03 & 0.23 & 0.23 & 14.30 \\
\hline Oxygen & 3.08 & 5.24 & 5.24 & 9.73 & 0.91 & 2.49 & 0.00 \\
\hline Chlorine & 10.19 & 13.65 & 2.68 & 2.68 & 0.50 & 2.42 & 0.03 \\
\hline Sulfur & 0.58 & 0.58 & 0.58 & 2.44 & 1.14 & 0.77 & 0.00 \\
\hline Nitrogen & 0.03 & $\underline{0.03}$ & 0.03 & 0.03 & 0.03 & 0.57 & 0.00 \\
\hline TOTAL & 100,00 & $\underline{100,00}$ & $\underline{100.00}$ & $\underline{\underline{100,00}}$ & 100.00 & $\underline{\underline{100,00}}$ & 100.00 \\
\hline
\end{tabular}


for this waste. It is definitely in the DOE interest to further the development and testing of the ThermoChem system and accelerate its commercial deployment. The next logical step toward meeting that objective is to conduct detailed and longer term tests on representative materials using hazardous compounds and radioactive surrogates. The $P C B$ contaminated waste at Portsmouth represents a good choice because of the large quantities at Portsmouth and other sites and the lack of other treatment options. Since several DOE LLMW materials also contain mercury compounds a surrogate test on a material containing mercury is also needed. Mercury was not included in the original surrogates to be tested under the DOE contract.

Through site visits and inquiries, ThermoChem has obtained information on the detailed physical, chemical, and radioactive characteristics of PCB-and Uranium-contaminated LLMW located at the Portsmouth Gaseous Diffusion Plant. Based on this data. ThermoChem has formulated a seventh surrogate waste stream for testing in the PDU. The feedstock composition is given in Table 3. The bulk ingredients are sorbent (polypropylene absorbent pads) and oil, the RCRA organic is monochlorobenzene (surrogate for PCB), and the radionuclide is a mixture of cerium fluoride $\left(\mathrm{CeF}_{3}\right)$ and Cerium oxide ( $\mathrm{CeO}$ ) (surrogates for Uranyl fluoride $\left(\mathrm{UO}_{2} \mathrm{~F}_{2}\right.$ ), Uranium Penta/tetrafluoride $\left(U F_{5} / U F_{4}\right)$ and Uranium oxides). Predominantly, it is planned to use the nominal composition. Two tests (HIGHI and HIGH2) of 8-hour duration each are planned with high concentrations of monochlorobenzene and cerium compounds to very accurately quantify (i) the Destruction and Removal Efficiency (DRE) of monochlorobenzene (to 6 nines or better), and (ii) cerium retention in the system (4 nines or better). Three short tests of 8 hours each are also planned (MERC1, MERC2 and MERC3) to investigate mercury retention in the system. The three mercury compounds to be tested are elemental mercury, mercury in reduced form - mercurous chloride, and mercury in oxidized form - mercury oxide. The ultimate analysis for the nominal composition is furnished in Table 2.

The preliminary design specifications for the surrogate waste treatment Process Development Unit (PDU) are given in Table 4. The design waste feed rate now is $90 \mathrm{lb} / \mathrm{hr}$. This scale provides the following advantages: 


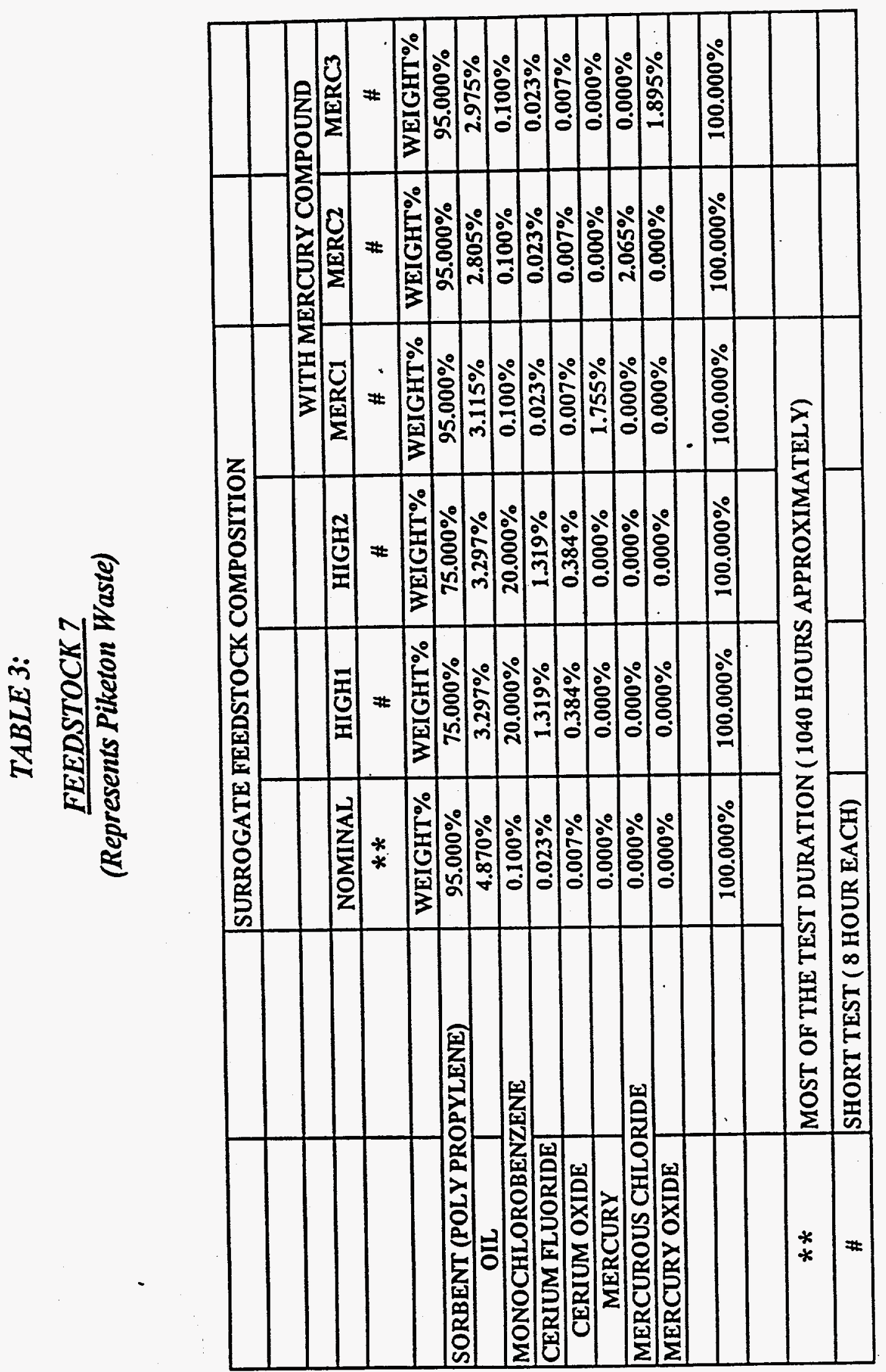




\section{TABLE 4:}

\section{PRELIMINARY DESIGN SPECIFICATIONS FOR THE SURROGATE WASTE TREATMENT PDU}

Design waste feed rate

Glass frit feed rate

Steam feed rate

Natural gas feed rate

Electrical heat input

Superficial fluidization velocity

First-stage steam reformer temperature

First-stage reformer bed material mean size

First-stage bed drain rate

Steam superheat temperature

Total steam load

$\mathrm{O}_{2}$ flow rate into second-stage reformer

Second-stage reformer temperature

Air flow rate into thermal oxidizer

Thermal oxidizer temperature

Quench water flow rate

Soda ash feed rate

HEPA filter catch rate

Pulse air flow rate

ID fan inlet temperature

ID fan flue gas flow rate
$90 \mathrm{lb} / \mathrm{hr}$

9-46 Ib/hr

$36-68 \mathrm{lb} / \mathrm{hr}$

$23-54 \mathrm{lb} / \mathrm{hr}$

- 34-63 kW

$1.6 \mathrm{ft} / \mathrm{s}$

$1000-1200^{\circ} \mathrm{F}$

150 - 250 microns

$16-92 \mathrm{lb} / \mathrm{hr}$

$900-1080^{\circ} \mathrm{F}$

$78-108 \mathrm{lb} / \mathrm{hr}$

$62-87 \mathrm{lb} / \mathrm{hr}$

$1800-2000^{\circ} \mathrm{F}$

$1235-1529 \mathrm{lb} / \mathrm{hr}$

$\sim 2000^{\circ} \mathrm{F}$

$693-869 \mathrm{lb} / \mathrm{hr}$

$3-18 \mathrm{lb} / \mathrm{hr}$

$7-27 \mathrm{lb} / \mathrm{hr}$

$144-180 \mathrm{lb} / \mathrm{hr}$

$215-233^{\circ} \mathrm{F}$

2273-2842 lb/hr

(515-644 scfm) 
- Thermal inertia of the system due to refractory is reduced. The refractory thickness depends on system operating temperature and not on the feed rate of the surrogate.

- Accurate material and heat balances at low waste feed rates due to lower system surface area-to-volume ratio.

- Commercially available, proven hot gas clean-up system matches this waste treatment scale.

The surrogate waste thermal treatment simulation summary of the six feedstocks are presented in Tables 5 through 10. The glass frit feed rate is varied to match the ash content of the waste feed. Based on communications with the vitrification experts, the proportion of waste residue in glass is typically known to lie in the 10 to 35 percent range by weight. The steam and natural gas feed rates are adjusted to maintain satisfactory fluidization velocity $(>1.5 \mathrm{ft} / \mathrm{s})$, and reasonable steam-to-carbonmass ratio $(>2)$. The Thermatrix system can process gas down to $10 \mathrm{Btu} / \mathrm{scf}$ but the system will typically be operated in the $150 \mathrm{Btu} / \mathrm{scf}$ range. Simulations were performed for three different Stage 1 steam reformer temperatures, viz. 1000,1100 , and $1200^{\circ} \mathrm{F}$. The Stage 2 steam reformer and the flameless thermal oxidizer temperatures were kept constant at $2000^{\circ} \mathrm{F}$. The $\mathrm{Na}$ to $(\mathrm{Cl}+\mathrm{S})$ molar feed ratio was nominally set at 1.25 . The quench water flow rate was varied to yield a HEPA filter inlet temperature of $250^{\circ} \mathrm{F}$. The volumetric flow rate estimates given in Tables 4 through 9 indicate that the waste volume reduction increases with a decrease in the ash content of the feedstock (see Table 2). Of course, the chlorine and sulfur content also influence the volume reduction. Feedstock 6 exhibits the greatest overall volume reduction (about 6 to 1) while Feedstock 1 results in the least overall volume reduction. The steam reformer system serves to separate and treat the hazardous constituents going into the melter/vitrifier so as to increase its throughput, and maximizes the Destruction and Removal Efficiency (DRE).

A process flow diagram for the PDU screening tests is given in Figure 2. The process flow sheets for the tests with feedstock 7 are furnished in Table 11. This corresponds to the nominal composition listed in Table 3. The simulations correspond to no sand addition during the test run. The feedstock 7 treatment simulation summary is presented in Table 12. 
TABLE 5:

SURROGATE WASTE THERMAL TREATMENT SIMULATION SUMMARY

FEEDSTOCK NO.

1

WASTE FEED RATE, PPH $\quad 90$

GLASS FRIT FEED RATE, PPH

STEAM FEED RATE TO STAGE 1 STEAM REFORMER, PPH

NATURAL GAS FEED RATE TO STAGE 1 STEAM REFORMER, PPH 36
Stage 1 Steam Reformer Off Gas, lb/hr

Stage 1 Steam Reformer Drain, lb/hr

Stage 1 Steam Reformer Drain, $\mathrm{ft}^{3} / \mathrm{hr}$

Stage 1 Steam Reformer Heat Input, $\mathrm{kW}$

Stage 1 Steam Heating Load, KBtu/hr

Stage 1 Back Pulse Steam, lb/hr

Stage 2 Steam Reformer $\mathrm{O}_{2}$ Flow Rate, lb/hr

Stage 2 Steam Reformer Product Gas Flow, lb/hr

Oxidizer Air Flow Rate, $\mathrm{lb} / \mathrm{hr}$

Oxidizer Flue Gas Flow Rate, lb/hr

Quench Water Flow Rate, lb/hr

Pulse Air Flow Rate, lb/hr

ID Fan Flue Gas Flow Rate, Ib/hr

ID Fan Inlet Temperature, ${ }^{\circ} \mathrm{F}$

ID Fan Flue Gas Flow Rate, scfm

Soda Ash Feed Rate, lb/hr

$\mathrm{NaCl}$ Produced, $\mathrm{lb} / \mathrm{hr}$

$\mathrm{Na}_{2} \mathrm{SO}_{4}$ Produced, $\mathrm{lb} / \mathrm{hr}$

Final HEPA Filter Solids Catch $\mathrm{lb} / \mathrm{hr}$

Total Steam Load, lb/hr

Waste Volume Feed Rate, lb/hr

Glass Frit Volume Feed Rate, $\mathrm{ft}^{3} \mathrm{hr}$

Steam Reformer Drain Rate, $\mathrm{ft}^{3} / \mathrm{hr}$

HEPA Filter Catch Rate, $\mathrm{ft}^{3} / \mathrm{hr}$
STEAM REFORMER TEMPERATURE, ${ }^{\circ} \mathrm{F}$

$\begin{array}{rrr}1000 & 1100 & 1200 \\ 133 & 134 & 135 \\ 92 & 91 & 91 \\ 1.03 & 1.02 & 1.01 \\ 38 & 41 & 44 \\ 78 & 80 & 82 \\ 9 & 9 & 9 \\ 64 & 63 & 63 \\ 206 & 206 & 206 \\ 1310 & 1338 & 1364 \\ 1516 & 1544 & 1570 \\ 720 & 732 & 743 \\ 152 & 154 & 157 \\ 2378 & 2420 & 2460 \\ 219 & 219 & 219 \\ 539 & 549 & 558 \\ 13.4 & 13.6 & 13.7 \\ 14.1 & 14.2 & 14.4 \\ 2.01 & 2.05 & 2.09 \\ 23.1 & 23.3 & 23.5 \\ 91 & 91 & 92 \\ 1.44 & 1.44 & 1.44 \\ 0.50 & 0.50 & 0.50 \\ 1.03 & 1.02 & 1.01 \\ 0.27 & 0.27 & 0.28\end{array}$


TABLE 6:

SURROGATE WASTE THERMAL TREATMENT SIMULATION SUMMARY

FEEDSTOCK NO.

2

WASTE FEED RATE, PPH

90

GLASS FRIT FEED RATE, PPH

18

STEAM FEED RATE TO STAGE 1 STEAM REFORMER, PPH

68

NATURAL GAS FEED RATE TO STAGE 1 STEAM REFORMER, PPH
Stage 1 Steam Reformer Off Gas, lb/hr

Stage 1 Steam Reformer Drain, $\mathrm{lb} / \mathrm{hr}$

Stage 1 Steam Reformer Drain, $\mathrm{ft}^{3} / \mathrm{hr}$

Stage 1 Steam Reformer Heat Input, $\mathrm{kW}$

Stage 1 Steam Heating Load, KBtu/hr

Stage 1 Back Pulse Steam, lb/hr

Stage 2 Steam Reformer $\mathrm{O}_{2}$ Flow Rate, $\mathrm{lb} / \mathrm{hr}$

Stage 2 Steam Reformer Product Gas Flow, lb/hr

Oxidizer Air Flow Rate, lb/hr

Oxidizer Flue Gas Flow Rate, lb/hr

Quench Water Flow Rate, lb/hr

Pulse Air Flow Rate, lb/hr

ID Fan Flue Gas Flow Rate, lb/hr

ID Fan Inlet Temperature, ${ }^{\circ} \mathrm{F}$

ID Fan Flue Gas Flow Rate, scfm

Soda Ash Feed Rate, lb/hr

$\mathrm{NaCl}$ Produced, $\mathrm{lb} / \mathrm{hr}$

$\mathrm{Na}_{2} \mathrm{SO}_{4}$ Produced, $\mathrm{lb} / \mathrm{hr}$

Final HEPA Filter Solids Catch, lb/hr

Total Steam Load, lb/hr

Waste Volume Feed Rate, lb/hr

Glass Frit Volume Feed Rate, $\mathrm{ft}^{3} \mathrm{hr}$

Steam Reformer Drain Rate, $\mathrm{ft}^{3} / \mathrm{hr}$

HEPA Filter Catch Rate, $\mathrm{ft}^{3} / \mathrm{hr}$
STEAM REFORMER TEMPERATURE, ${ }^{\circ} \mathrm{F}$

$\begin{array}{rrr}1000 & 1100 & 1200 \\ 151 & 153 & 155 \\ 46 & 45 & 43 \\ 0.51 & 0.50 & 0.48 \\ 55 & 59 & 63 \\ 97 & 100 & 103 \\ 9 & 9 & 9 \\ 63 & 63 & 62 \\ 224 & 225 & 225 \\ 1392 & 1443 & 1492 \\ 1616 & 1668 & 1717 \\ 764 & 786 & 808 \\ 162 & 167 & 172 \\ 2533 & 2613 & 2688 \\ 215 & 215 & 216 \\ 574 & 592 & 610 \\ 17.5 & 17.7 & 17.9 \\ 18.8 & 19.0 & 19.2 \\ 2.01 & 2.05 & 2.09 \\ 25.8 & 26.1 & 26.4 \\ 106 & 107 & 108 \\ 1.44 & 1.44 & 1.44 \\ 0.20 & 0.20 & 0.20 \\ 0.51 & 0.50 & 0.48 \\ 0.31 & 0.31 & 0.31\end{array}$


TABLE 7:

SURROGATE WASTE THERMAL TREATMENT SIMULATION SUMMARY

FEEDSTOCK NO. 3

WASTE FEED RATE, PPH

90

GLASS FRIT FEED RATE, PPH

46

STEAM FEED RATE TO STAGE 1 STEAM REFORMER, PPH

54

NATURAL GAS FEED RATE TO STAGE I STEAM REFORMER, PPH

36

Stage 1 Steam Reformer Off Gas, Ib/hr

Stage 1 Steam Reformer Drain, $\mathrm{lb} / \mathrm{hr}$

Stage 1 Steam Reformer Drain, $\mathrm{ft}^{3} / \mathrm{hr}$

Stage 1 Steam Reformer Heat Input, $\mathrm{kW}$

Stage 1 Steam Heating Load, KBtu/hr

Stage 1 Back Pulse Steam, lb/hr

Stage 2 Steam Reformer $\mathrm{O}_{2}$ Flow Rate, $\mathrm{lb} / \mathrm{hr}$

Stage 2 Steam Reformer Product Gas Flow, lb/hr

Oxidizer Air Flow Rate, $\mathrm{lb} / \mathrm{hr}$

Oxidizer Flue Gas Flow Rate, lb/hr

Quench Water Flow Rate, lb/hr

Pulse Air Flow Rate, lb/hr

ID Fan Flue Gas Flow Rate, lb/hr

ID Fan Inlet Temperature, ${ }^{\circ} \mathrm{F}$

ID Fan Flue Gas Flow Rate, scfm

Soda Ash Feed Rate, lb/hr

$\mathrm{NaCl}$ Produced, $\mathrm{lb} / \mathrm{hr}$

$\mathrm{Na}_{2} \mathrm{SO}_{4}$ Produced, $\mathrm{lb} / \mathrm{hr}$

Final HEPA Filter Solids Catch, lb/hr

Total Steam Load, lb/hr

Waste Volume Feed Rate, $\mathrm{lb} / \mathrm{hr}$

Glass Frit Volume Feed Rate, $\mathrm{f}^{3} \mathrm{hr}$

Steam Reformer Drain Rate, $\mathrm{ft}^{3} / \mathrm{hr}$

HEPA Filter Catch Rate, $\mathrm{A}^{3} / \mathrm{hr}$
STEAM REFORMER TEMPERATURE ${ }^{\circ} \mathrm{F}$

1000

134

1100

135

91

1.01

1.02

34

78

9

65

208

1235

1443

693

144

2273

231

515

4.6

3.7

2.01

11.1

90

1.44

0.51

1.02

0.12
38

80

9

64

208

1261

1468

703

147

2312

231

524

4.6

3.7

2.05

11.2

90

1.44

0.51

1.01

0.13
1200

135

90

1.00

41

82

9

63

207

1285

1492

714

149

2349

231

533

4.7

3.8

2.09

11.2

90

1.44

0.51

1.00

0.13 


\section{TABLE 8:}

\section{SURROGATE WASTE THERMAL TREATMENT SIMULATION SUMMARY}

FEEDSTOCK NO.

4

WASTE FEED RATE, PPH GLASS FRTT FEED RATE, PPH

STEAM FEED RATE TO STAGE 1 STEAM REFORMER, PPH

NATURAL GAS FEED RATE TO STAGE 1 STEAM REFORMER, PPH
90

26

59

31
Stage 1 Steam Reformer Off Gas, lb/hr

Stage 1 Steam Reformer Drain, lb/hr

Stage 1 Steam Reformer Drain, $\mathrm{ft}^{3} / \mathrm{hr}$

Stage 1 Steam Reformer Heat Input, $\mathrm{kW}$

Stage 1 Steam Heating Load, KBtw/hr

Stage 1 Back Pulse Steam, lb/hr

Stage 2 Steam Reformer $\mathrm{O}_{2}$ Flow Rate, $\mathrm{lb} / \mathrm{hr}$

Stage 2 Steam Reformer Product Gas Flow, lb/hr

Oxidizer Air Flow Rate, $\mathrm{lb} / \mathrm{hr}$

Oxidizer Flue Gas Flow Rate, lb/hr

Quench Water Flow Rate, lb/hr

Pulse Air Flow Rate, $\mathrm{lb} / \mathrm{hr}$

ID Fan Flue Gas Flow Rate, lb/hr

ID Fan Inlet Temperature, ${ }^{\circ} \mathrm{F}$

ID Fan Flue Gas Flow Rate, scfm

Soda Ash Feed Rate, lb/hr

$\mathrm{NaCl}$ Produced, lb/hr

$\mathrm{Na}_{2} \mathrm{SO}_{4}$ Produced, $\mathrm{lb} / \mathrm{hr}$

Final HEPA Filter Solids Catch, lb/hr

Total Steam Load, lb/hr

Waste Volume Feed Rate, $\mathrm{lb} / \mathrm{hr}$

Glass Frit Volume Feed Rate, $\mathrm{f}^{3} / \mathrm{hr}$

Steam Reformer Drain Rate, $\mathrm{ft}^{3} / \mathrm{hr}$

HEPA Filter Catch Rate, $\mathrm{ft}^{3} / \mathrm{hr}$

\section{STEAM REFORMER TEMPERATURE ${ }^{\circ} \mathrm{F}$}

$1000 \cdot 1100 \quad 1200$

147

59

148

150

0.65

58

56

0.63

43

84

9

67

223

1408

1631

775

163

2562

226

581

0.64

47

51

87

89

9

66

9

65

224

1488

1712

810

171

2686

226

609

9.1

3.7

8.47

16.4

97

1.44

0.29

0.65

0.17 
TABLE 9:

SURROGATE WASTE THERMAL TREATMENT SIMULATION SUMMARY

FEEDSTOCK NO. '

WASTE FEED RATE, PPH 90

GLASS FRTT FEED RATE, PPH 46

STEAM FEED RATE TO STAGE I STEAM REFORMER, PPH 50

NATURAL GAS FEED RATE TO STAGE 1 STEAM REFORMER, PPH 40

Stage 1 Steam Reformer Off Gas, $\mathrm{lb} / \mathrm{hr}$

Stage 1 Steam Reformer Drain, lb/hr

Stage 1 Steam Reformer Drain, $\mathrm{ft}^{3} / \mathrm{hr}$

Stage 1 Steam Reformer Heat Input, $\mathrm{kW}$

Stage 1 Steam Heating Load, Kbtu/hr

Stage 1 Back Pulse Steam, lb/hr

Stage 2 Steam Reformer $\mathrm{O}_{2}$ Flow Rate, $\mathrm{lb} / \mathrm{hr}$

Stage 2 Steam Reformer Product Gas Flow, lb/hr

Oxidizer Air Flow Rate, lb/hr

Oxidizer Flue Gas Flow Rate, lb/hr

Quench Water Flow Rate, lb/hr

Pulse Air Flow Rate, lb/hr

ID Fan Flue Gas Flow Rate, lb/hr

ID Fan Inlet Temperature, ${ }^{\circ} \mathrm{F}$

ID Fan Flue Gas Flow Rate, scfm

Soda Ash Feed Rate, lb/hr

$\mathrm{NaCl}$ Produced, $\mathrm{lb} / \mathrm{hr}$

$\mathrm{Na}_{2} \mathrm{SO}_{4}$ Produced, $\mathrm{lb} / \mathrm{hr}$

Final HEPA Filter Solids Catch, $\mathrm{lb} / \mathrm{hr}$

Total Steam Load, lb/hr

Waste Volume Feed Rate, lb/hr

Glass Frit Volume Feed Rate, $\mathrm{f}^{3} \mathrm{hr}$

Steam Reformer Drain Rate, $\mathrm{ft}^{3} / \mathrm{hr}$

HEPA Filter Catch Rate, $\mathrm{ft}^{3} / \mathrm{hr}$
STEAM REFORMER TEMPERATURE, ${ }^{\circ} \mathrm{F}$

$\begin{array}{rrr}1000 & 1100 & 1200 \\ 135 & 135 & 136 \\ 92 & 91 & 91 \\ 1.02 & 1.01 & 1.01 \\ 40 & 43 & 46 \\ 71 & 74 & 76 \\ 9 & 9 & 9 \\ 67 & 66 & 66 \\ 211 & 210 & 210 \\ 1242 & 1262 & 1281 \\ 1452 & 1472 & 1291 \\ 699 & 707 & 716 \\ 145 & 147 & 149 \\ 2290 & 2320 & 2350 \\ 233 & 233 & 233 \\ 519 & 526 & 533 \\ 3.4 & 3.4 & 3.5 \\ 0.7 & 0.7 & 0.7 \\ 3.95 & 4.03 & 4.11 \\ 9.9 & 10.0 & 10.1 \\ 85 & 86 & 86 \\ 1.44 & 1.44 & 1.44 \\ 0.52 & 0.52 & 0.52 \\ 1.02 & 1.01 & 1.01 \\ 0.10 & 0.10 & 0.10\end{array}$


TABLE 10:

SURROGATE WASTE THERMAL TREATMENT SIMULATION SUMMARY

FEEDSTOCK NO.

6

WASTE FEED RATE, PPH

90

GLASS FRTT FEED RATE, PPH

9

STEAM FEED RATE TO STAGE 1 STEAM REFORMER, PPH

36

NATURAL GAS FEED RATE TO STAGE 1 STEAM REFORMER, PPH

54

Stage 1 Steam Reformer Off Gas, lb/hr

Stage 1 Steam Reformer Drain, lb/hr

Stage 1 Steam Reformer Drain, $\mathrm{ft}^{3} / \mathrm{hr}$

Stage 1 Steam Reformer Heat Input, Kw

Stage 1 Steam Heating Load, Kbtu/hr

Stage 1 Back Pulse Steam, lb/hr

STEAM REFORMER TEMPERATURE 。 ${ }^{\circ}$

Stage 2 Steam Reformer $\mathrm{O}_{2}$ Flow Rate, $\mathrm{lb} / \mathrm{hr}$

Stage 2 Steam Reformer Product Gas Flow, lb/hr

Oxidizer Air Flow Rate, lb/hr

Oxidizer Flue Gas Flow Rate, lb/hr

Quench Water Flow Rate, lb/hr

Pulse Air Flow Rate, lb/hr

ID Fan Flue Gas Flow Rate, lb/hr

ID Fan Inlet Temperature, ${ }^{\circ} \mathrm{F}$

ID Fan Flue Gas Flow Rate, scfm

Soda Ash Feed Rate, lb/hr

$\mathrm{NaCl}$ Produced, lb/hr

$\mathrm{Na}_{2} \mathrm{SO}_{4}$ Produced, $\mathrm{lb} / \mathrm{hr}$

Final HEPA Filter Solids Catch, lb/hr

Total Steam Load, $1 \mathrm{~b} / \mathrm{hr}$

Waste Volume Feed Rate, lb/hr

Glass Frit Volume Feed Rate, $\mathrm{ft}^{3} / \mathrm{hr}$

Steam Reformer Drain Rate, $\mathrm{t}^{3} / \mathrm{hr}$

HEPA Filter Catch Rate, $\mathrm{ft}^{3} / \mathrm{hr}$

$\begin{array}{rrr}1000 & 1100 & 1200 \\ 173 & 173 & 173 \\ 16 & 16 & 16 \\ 0.18 & 0.18 & 0.18 \\ 45 & 48 & 50 \\ 52 & 53 & 55 \\ 9 & 9 & 9 \\ 87 & 86 & 85 \\ 268 \quad 268 & 267 & \\ 1493 & 1511 & 1529 \\ 1761 & 1779 & 1796 \\ 854 & 862 & 869 \\ 176 & 178 & 180 \\ 2789 & 2815 & 2842 \\ 232 & 232 & 232 \\ 632 & 638 & 644 \\ 4.7 & 4.8 & 4.9 \\ 3.3 & 3.4 & 3.4 \\ 2.69 & 2.75 & 2.80 \\ 7.6 & 7.8 & 7.8 \\ 78 & 78 & 78 \\ 1.44 & 1.44 & 1.44 \\ 0.10 & 0.10 & 0.10 \\ 0.18 & 0.18 & 0.18 \\ 0.08 & 0.09 & 0.09\end{array}$




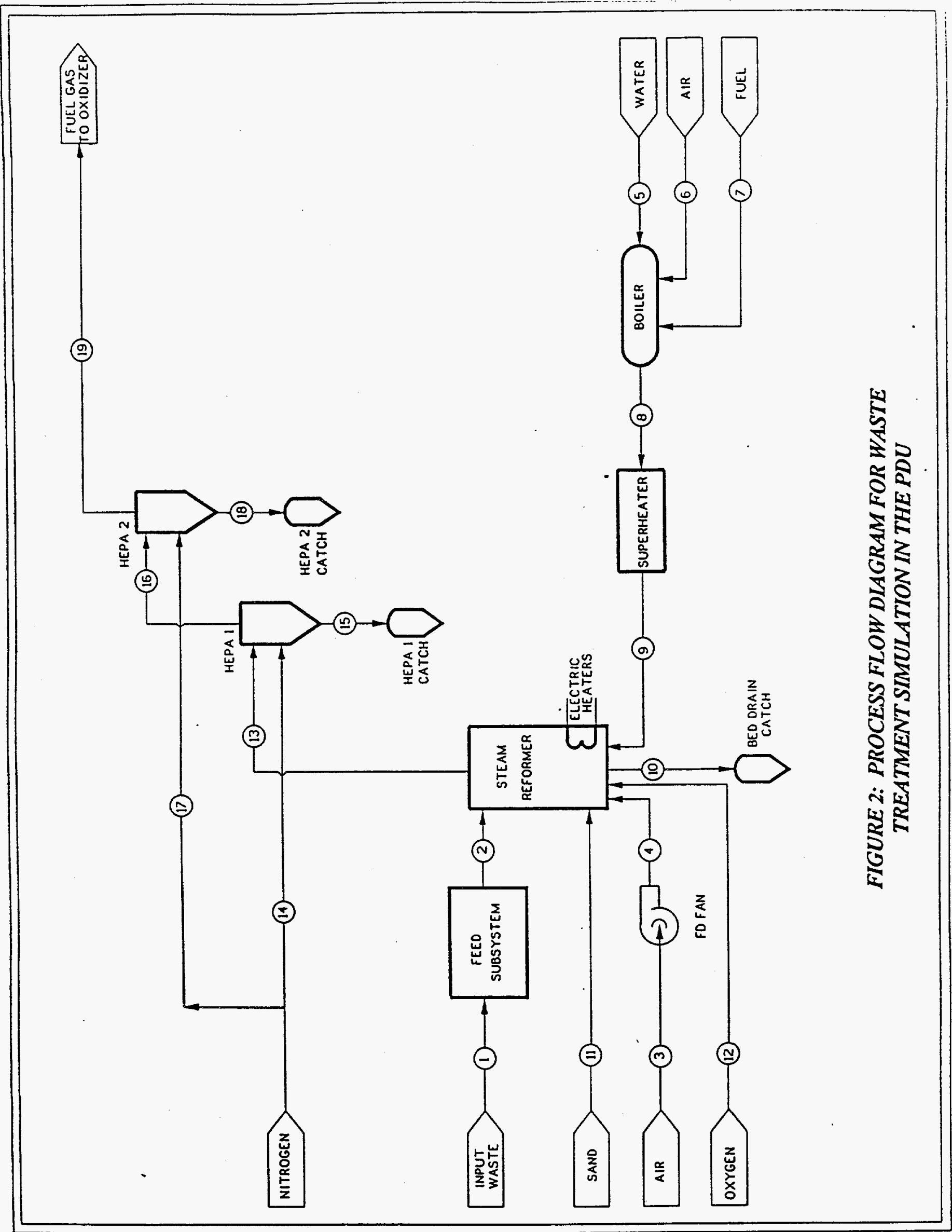




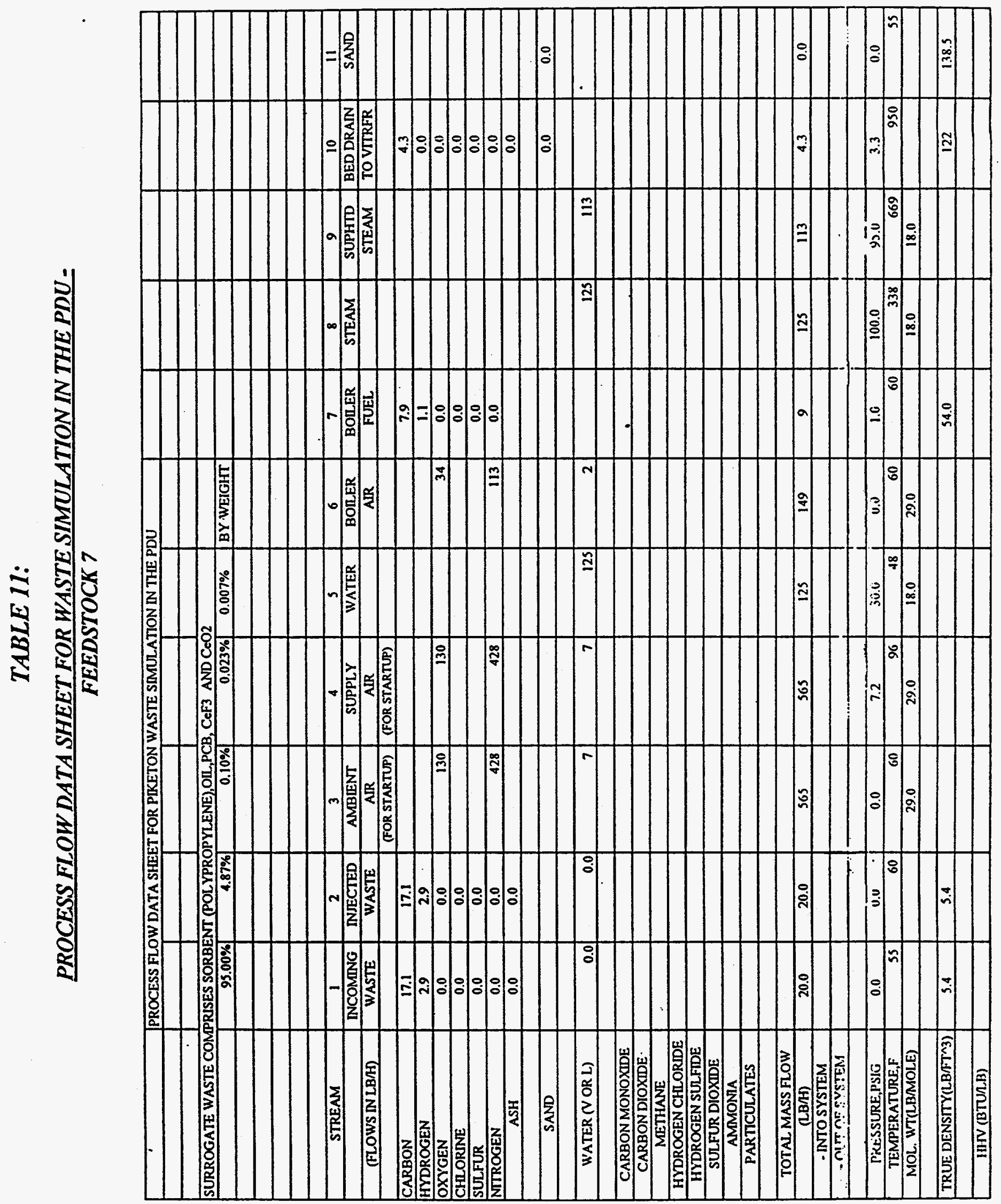




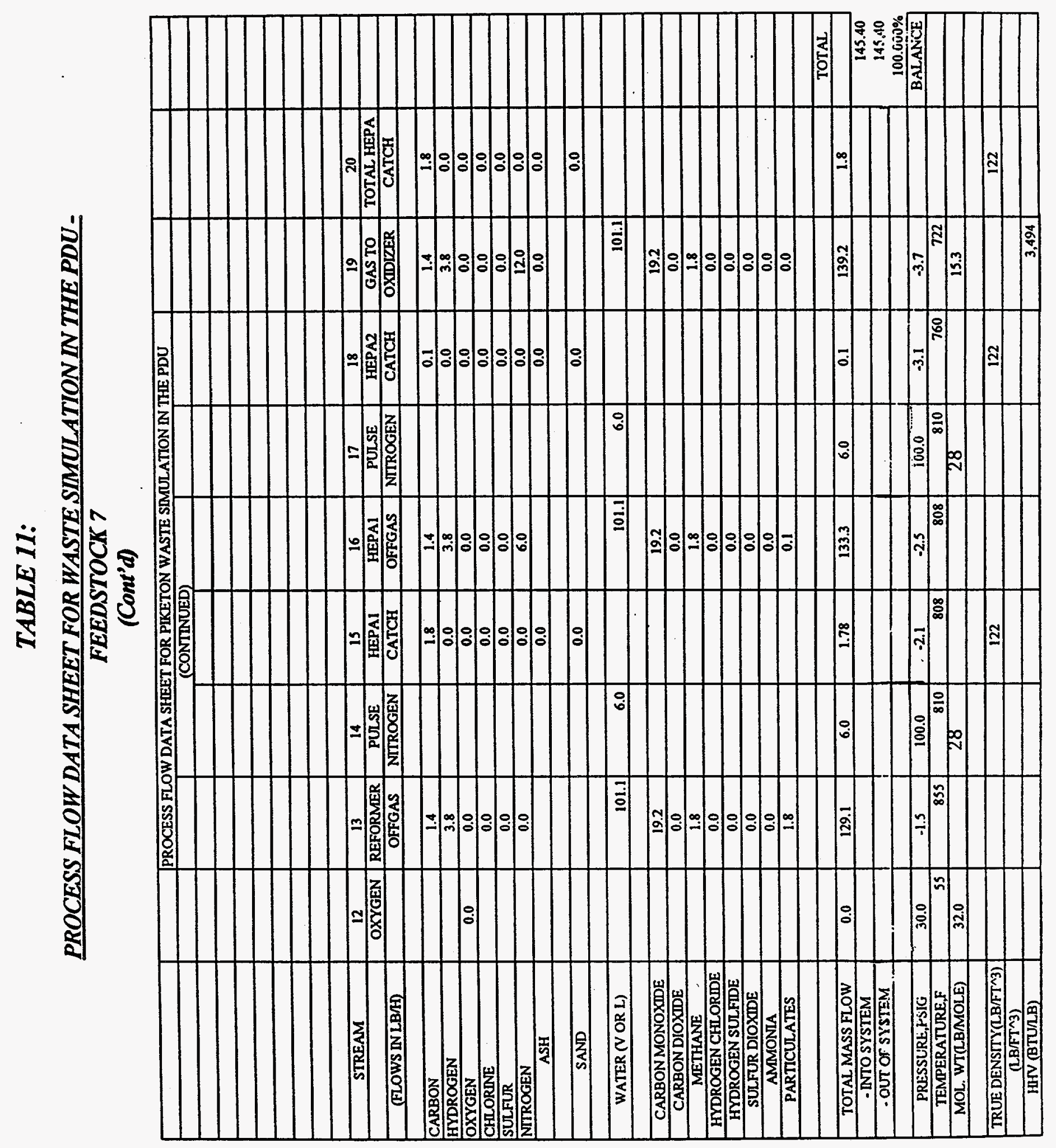


TABLE 12:

FEEDSTOCK 7 WASTE TREATMENT SIMULATION SUMARY

\begin{tabular}{|c|c|}
\hline \multicolumn{2}{|c|}{ PIKETON WASTE THERMAL TREATMENT SMULATION SUMDMARY } \\
\hline FEEDSTOCK & PREETON W. \\
\hline WASTE FEEDRATE,PPH & 20 \\
\hline SAND FEEDRATE,PPH & $\mathbf{0}$ \\
\hline STEAM FEEDRATE TO STAGE I SR,PPH & 113 \\
\hline STAGE I STM REF TEMP,F & 950 \\
\hline STAGE I STEAM REFRMR OFFGAS,LB/HR & 129 \\
\hline STAGE I STEAM REFRMR DRAN,LB/HR & 4.3 \\
\hline STAGE 1 STM REFRMR DRAIN,FT^3/HR & 0.06 \\
\hline STAGE I STM REFRMR HEAT INPUT,kW & 45 \\
\hline STAGE I STEAM HTNG LOAD,kBTU/HR & 148 \\
\hline ST I STM FOR SOLDS TRNSPRT,LB/HR & $\mathbf{0}$ \\
\hline OXYGEN TO REFORMER,LB/HR & $\overline{0}$ \\
\hline HTHEPAI\&2 BACK PULSE STEAMLLBAR & 12 \\
\hline HTHEPA OFFGAS RATE,LB/TR & 139 \\
\hline HTHEPA OFFGAS MOL WEIGHT,LBAMOLE & 15.3 \\
\hline HTHEPA OFFGAS HHV,BTU/LB & $\overline{3,494}$ \\
\hline HTHEPA OFFGAS LHV,BTURB & 2,446 \\
\hline HTHEPA OFFGAS PRESSURE,PSIG & -3.7 \\
\hline HTHEPA OFFGAS TEMPERATURE,F & 722 \\
\hline HTHEPA OFFGAS VOLFLO RATE,ACFM & 174 \\
\hline HTHEPAI \&2 SOLDS CATCH RATE,LB/HR & 1.8 \\
\hline MELTER FEED RATE,LB/HR & 6.2 \\
\hline MASS REDUCTION RATIO & 3.2 \\
\hline STAGE 2 SR GAS FRR RATE, MQMBTU/HR & 0 \\
\hline STAGE 2 SR GAS FLCW RATE,LB/HR & 0 \\
\hline TEMPERNG WATER FLOW RATE,LB/HR & 1 \\
\hline OXIIIZER PRODUCT GAS INFLOW,LB/HR & 140 \\
\hline BURNER GAS FRRNG RATE,KBTU/HR & - \\
\hline BURNER GAS FLOW RATELB/ER & 0.0 \\
\hline OXDZR AIR FLOW RATE,LBARR & 3.286 \\
\hline OXDZR FLUEGAS FLOW RATE,LB/HR & 3,426 \\
\hline OXDZR FLUEGAS FLOW RATE,SCFM & 805 \\
\hline QUENCH WATER FLOW RATE,LB/HR & 817 \\
\hline PULSE AIR FLOW RATE,LB/HR & 343 \\
\hline D FAN FLUE GAS FLOW RATE.LB/HR & 4,586 \\
\hline D FAN DLET TEMPERATURE,F & 144 \\
\hline D FAN FLUEGAS FLOW RATE,SCFM & 1,180 \\
\hline D FAN EXTT PRESSURE,PSIG & 0.3 \\
\hline D FAN EXTT TEMPERATURE,F & 349 \\
\hline D FAN FLUEGAS EXIT VFRATE,ACFM & 1,742 \\
\hline SODA ASH FEED RATE,LB/FR & 0.0 \\
\hline NACL PRODUCED,LB/RR & 0.0 \\
\hline NA2SO4 PRODUCED,LB/HR & 0.0 \\
\hline FWNAL HEPAF SOLIDS CATCHLLB/HR & 0.0 \\
\hline TOTAL STEAMLOAD FOR REFORMER LBAR & 157 \\
\hline WASTE VOLUME FEED RATE,FT^3/HR & 3.69 \\
\hline SAND VOL FEED RATE,FT^3/HR & 0.00 \\
\hline STM REF DRANN RATE,FT^3/RR & 0.06 \\
\hline HTHEPAI\&2 DRAN RATE,FT^3/HR & 0.03 \\
\hline MELTER FEED RATE,FT^3/AR & 0.09 \\
\hline HEPAF CATCH RATE,FT^3/RR & 0.000 \\
\hline VOLUME REDUCTION & 42 \\
\hline
\end{tabular}


It is believed that the steam reformer system arrangement shown in Figure 1 is capable of achieving 99.9999\% DRE for the Principal Organic Hazardous Constituents (POHCs). For the first six feedstocks, the POHCs of relevance here are naphthalene, dichlorobenzene, toluene, phenol, tetrachloroethylene, vinyl chloride, and ethylene glycol. In order to determine the measurement/ instrument accuracy needed to monitor these compounds in the flue gas stream, the concentrations of two POHCs, viz. naphthalene and dichlorobenzene, in the flue gas by volume were estimated for the six feedstocks as a function of the DRE. The results are shown in Tables 13 through 18 . For weight percent in feed between 0.5 and 10 and for DRE greater than 99.99 percent, the concentration of POHCs in flue gas ranges between 0.01 and $45 \mathrm{ppb}$. Based on conversations with a Centre Analytical Laboratory scientist, the minimum concentration measurable is on the order of $1 \mathrm{ppb}$. Radian Corporation was also contacted to determine the instrument least count and their response is given in Table 19. The minimum detection limit tums out to be about $0.3 \mathrm{ppb}$. Based on these findings, it may be necessary to increase the weight percent in feed of some of the POHCs in order to accurately determine DRE values.

In the case of feedstock 7, a majority of the tests will be conducted with the nominal composition (see Table 3) which closely resembles the actual Piketon waste. Five short test rums of 8 hours duration each, however, are planned with much higher concentrations of one or more of the following compounds - monochlorobenzene, cerium fluoride, cerium oxide, mercury, mercurous chloride, and mercury oxide - to verify greater than 99.9999\% DRE for monochlorobenzene and greater than $99.99 \%$ retention of cerium and mercury in the solids.

For the first six feedstocks, the program requires at least 500 hours of operations be performed with at least 180 hours of feed time at a rate of 90 pounds per hour. The 500 hours of operation should include 320 hours of preheat and cool-down between each test. The program plan is to perform 12 operational tests of 8 to 20 hours feed time per test. Approximately one test will be performed each week with the PDU cleaned and inspected between each test. A 13th test may be performed to treat the remaining unused feedstock and other appropriate material to provide a long duration run of 48 or 72 hours. 
TABLE 13:

POHC CONCENTRATION IN FLUE GAS

\section{FEEDSTOCK NO. 1}

\section{FLUE GAS CONCENTRATION}

Wt.\%

In Feed
DRE

$\begin{array}{lllll}\underline{0.990000} & \underline{0.999000} \quad \underline{0.999900} \quad \underline{0.999990} \quad \underline{0.999999}\end{array}$

$\begin{array}{rrrrrrr}0.5 & 1.4 \mathrm{E}-06 & 2.2 \mathrm{E}-07 & 2.2 \mathrm{E}-08 & 2.2 \mathrm{E}-09 & 2.2 \mathrm{E}-10 & 2.2 \mathrm{E}-11 \\ 2.0 & 5.7 \mathrm{E}-06 & 8.7 \mathrm{E}-07 & 8.7 \mathrm{E}-08 & 8.7 \mathrm{E}-09 & 8.7 \mathrm{E}-10 & 8.7 \mathrm{E}-11 \\ 5.0 & 1.4 \mathrm{E}-05 & 2.2 \mathrm{E}-06 & 2.2 \mathrm{E}-07 & 2.2 \mathrm{E}-08 & 2.2 \mathrm{E}-09 & 2.2 \mathrm{E}-10 \\ 10.0 & 2.9 \mathrm{E}-05 & 4.3 \mathrm{E}-06 & 4.3 \mathrm{E}-07 & 4.3 \mathrm{E}-08 & 4.3 \mathrm{E}-09 & 4.3 \mathrm{E}-10\end{array}$

Dichlorobenzene

$\begin{array}{rrrrrrr}0.5 & 1.2 \mathrm{E}-06 & 1.9 \mathrm{E}-07 & 1.9 \mathrm{E}-08 & 1.9 \mathrm{E}-09 & 1.9 \mathrm{E}-10 & 1.9 \mathrm{E}-11 \\ 2.0 & 5.0 \mathrm{E}-06 & 7.6 \mathrm{E}-07 & 7.6 \mathrm{E}-08 & 7.6 \mathrm{E}-09 & 7.6 \mathrm{E}-10 & 7.6 \mathrm{E}-11 \\ 5.0 & 1.2 \mathrm{E}-05 & 1.9 \mathrm{E}-06 & 1.9 \mathrm{E}-07 & 1.9 \mathrm{E}-08 & 1.9 \mathrm{E}-09 & 1.9 \mathrm{E}-10 \\ 10.0 & 2.5 \mathrm{E}-05 & 3.8 \mathrm{E}-06 & 3.8 \mathrm{E}-07 & 3.8 \mathrm{E}-08 & 3.8 \mathrm{E}-09 & 3.8 \mathrm{E}-10\end{array}$




\section{TABLE 14:}

\section{POHC CONCENTRATION IN FLUE GAS}

\section{FEEDSTOCK NO. 2}

\section{FLUE GAS CONCENTRATION}

$\mathrm{Wt} . \%$

In Feed

Naphthalene

$\begin{array}{rrrrrrr}0.5 & 1.3 \mathrm{E}-06 & 2.0 \mathrm{E}-07 & 2.0 \mathrm{E}-08 & 2.0 \mathrm{E}-09 & 2.0 \mathrm{E}-10 & 2.0 \mathrm{E}-11 \\ 2.0 & 5.2 \mathrm{E}-06 & 8.0 \mathrm{E}-07 & 8.0 \mathrm{E}-08 & 8.0 \mathrm{E}-09 & 8.0 \mathrm{E}-10 & 8.0 \mathrm{E}-11 \\ 5.0 & 1.3 \mathrm{E}-05 & 2.0 \mathrm{E}-06 & 2.0 \mathrm{E}-07 & 2.0 \mathrm{E}-08 & 2.0 \mathrm{E}-09 & 2.0 \mathrm{E}-10 \\ 10.0 & 2.6 \mathrm{E}-05 & 4.0 \mathrm{E}-06 & 4.0 \mathrm{E}-07 & 4.0 \mathrm{E}-08 & 4.0 \mathrm{E}-09 & 4.0 \mathrm{E}-10\end{array}$

Dichlorobenzene

$\begin{array}{rrrrrrr}0.5 & 1.1 \mathrm{E}-06 & 1.7 \mathrm{E}-07 & 1.7 \mathrm{E}-08 & 1.7 \mathrm{E}-09 & 1.7 \mathrm{E}-10 & 1.7 \mathrm{E}-11 \\ 2.0 & 4.6 \mathrm{E}-06 & 7.0 \mathrm{E}-07 & 7.0 \mathrm{E}-08 & 7.0 \mathrm{E}-09 & 7.0 \mathrm{E}-10 & 7.0 \mathrm{E}-11 \\ 5.0 & 1.1 \mathrm{E}-05 & 1.7 \mathrm{E}-06 & 1.7 \mathrm{E}-07 & 1.7 \mathrm{E}-08 & 1.7 \mathrm{E}-09 & 1.7 \mathrm{E}-10 \\ 10.0 & 2.3 \mathrm{E}-05 & 3.5 \mathrm{E}-06 & 3.5 \mathrm{E}-07 & 3.5 \mathrm{E}-08 & 3.5 \mathrm{E}-09 & 3.5 \mathrm{E}-10\end{array}$

DRE

$\begin{array}{lllll}0.990000 & \underline{0.999000} \quad \underline{0.999900} \quad \underline{0.999990} \quad \underline{0.999999}\end{array}$

4.07

$4.0 \mathrm{E}-09$

$4.0 \mathrm{E}-10$
$4.0 \mathrm{E}-08$ 
TABLE 15:

POHC CONCENTRATION IN FLUE GAS

FEEDSTOCK NO. 3

\section{FLUE GAS CONCENTRATION}

Wt. \%

In Feed
DRE

$\begin{array}{lllll}\underline{0.990000} & \underline{0.999000} \quad \underline{0.999900} \quad \underline{0.999990} \quad \underline{0.999999}\end{array}$

$\begin{array}{rrrrrrr}0.5 & 1.5 \mathrm{E}-06 & 2.3 \mathrm{E}-07 & 2.3 \mathrm{E}-08 & 2.3 \mathrm{E}-09 & 2.3 \mathrm{E}-10 & 2.3 \mathrm{E}-11 \\ 2.0 & 6.0 \mathrm{E}-06 & 9.0 \mathrm{E}-07 & 9.0 \mathrm{E}-08 & 9.0 \mathrm{E}-09 & 9.0 \mathrm{E}-10 & 9.0 \mathrm{E}-11 \\ 5.0 & 1.5 \mathrm{E}-05 & 2.3 \mathrm{E}-06 & 2.3 \mathrm{E}-07 & 2.3 \mathrm{E}-08 & 2.3 \mathrm{E}-09 & 2.3 \mathrm{E}-10 \\ 10.0 & 3.0 \mathrm{E}-05 & 4.5 \mathrm{E}-06 & 4.5 \mathrm{E}-07 & 4.5 \mathrm{E}-08 & 4.5 \mathrm{E}-09 & 4.5 \mathrm{E}-10\end{array}$

Dichlorobenzene

$\begin{array}{rrrrrrr}0.5 & 1.3 \mathrm{E}-06 & 2.0 \mathrm{E}-07 & 2.0 \mathrm{E}-08 & 2.0 \mathrm{E}-09 & 2.0 \mathrm{E}-10 & 2.0 \mathrm{E}-11 \\ 2.0 & 5.2 \mathrm{E}-06 & 7.9 \mathrm{E}-07 & 7.9 \mathrm{E}-08 & 7.9 \mathrm{E}-09 & 7.9 \mathrm{E}-10 & 7.9 \mathrm{E}-11 \\ 5.0 & 1.3 \mathrm{E}-05 & 2.0 \mathrm{E}-06 & 2.0 \mathrm{E}-07 & 2.0 \mathrm{E}-08 & 2.0 \mathrm{E}-09 & 2.0 \mathrm{E}-10 \\ 10.0 & 2.6 \mathrm{E}-05 & 3.9 \mathrm{E}-06 & 3.9 \mathrm{E}-07 & 3.9 \mathrm{E}-08 & 3.9 \mathrm{E}-09 & 3.9 \mathrm{E}-10\end{array}$


TABLE 16:

\section{POHC CONCENTRATION IN FLUE GAS}

\section{FEEDSTOCK NO. 4}

\section{FLUE GAS CONCENTRATION}

Wt.\%

In Feed $\underline{0.990000}$

DRE

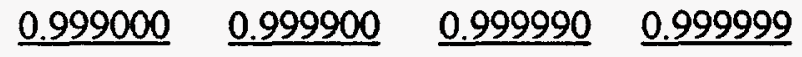

Naphthalene

$\begin{array}{rrrrrrr}0.5 & 1.3 \mathrm{E}-06 & 2.0 \mathrm{E}-07 & 2.0 \mathrm{E}-08 & 2.0 \mathrm{E}-09 & 2.0 \mathrm{E}-10 & 2.0 \mathrm{E}-11 \\ 2.0 & 5.2 \mathrm{E}-06 & 8.0 \mathrm{E}-07 & 8.0 \mathrm{E}-08 & 8.0 \mathrm{E}-09 & 8.0 \mathrm{E}-10 & 8.0 \mathrm{E}-11 \\ 5.0 & 1.3 \mathrm{E}-05 & 2.0 \mathrm{E}-06 & 2.0 \mathrm{E}-07 & 2.0 \mathrm{E}-08 & 2.0 \mathrm{E}-09 & 2.0 \mathrm{E}-10 \\ 10.0 & 2.6 \mathrm{E}-05 & 4.0 \mathrm{E}-06 & 4.0 \mathrm{E}-07 & 4.0 \mathrm{E}-08 & 4.0 \mathrm{E}-09 & 4.0 \mathrm{E}-10\end{array}$

Dichlorobenzene

$\begin{array}{rrrrrrr}0.5 & 1.1 \mathrm{E}-06 & 1.7 \mathrm{E}-07 & 1.7 \mathrm{E}-08 & 1.7 \mathrm{E}-09 & 1.7 \mathrm{E}-10 & 1.7 \mathrm{E}-11 \\ 2.0 & 4.6 \mathrm{E}-06 & 6.9 \mathrm{E}-07 & 6.9 \mathrm{E}-08 & 6.9 \mathrm{E}-09 & 6.9 \mathrm{E}-10 & 6.9 \mathrm{E}-11 \\ 5.0 & 1.1 \mathrm{E}-05 & 1.7 \mathrm{E}-06 & 1.7 \mathrm{E}-07 & 1.7 \mathrm{E}-08 & 1.7 \mathrm{E}-09 & 1.7 \mathrm{E}-10 \\ 10.0 & 2.3 \mathrm{E}-05 & 3.5 \mathrm{E}-06 & 3.5 \mathrm{E}-07 & 3.5 \mathrm{E}-08 & 3.5 \mathrm{E}-09 & 3.5 \mathrm{E}-10\end{array}$


TABLE 17:

POHC CONCENTRATION IN FLUE GAS

\section{FEEDSTOCK NO. 5}

\section{FLUE GAS CONCENTRATION}

Wt.\%

$\underline{\text { In Feed }}$

\begin{tabular}{lrrrrrrrr} 
& $\begin{array}{r}\text { Wt.\% } \\
\text { In Feed }\end{array}$ & & $\underline{0.990000}$ & $\underline{0.999000}$ & $\underline{0.999900}$ & $\underline{0.999990}$ & $\underline{0.999999}$ \\
\multirow{2}{*}{ Naphthalene } & 0.5 & $1.5 \mathrm{E}-06$ & $2.2 \mathrm{E}-07$ & $2.2 \mathrm{E}-08$ & $2.2 \mathrm{E}-09$ & $2.2 \mathrm{E}-10$ & $2.2 \mathrm{E}-11$ \\
& 2.0 & $6.0 \mathrm{E}-06$ & $9.0 \mathrm{E}-07$ & $9.0 \mathrm{E}-08$ & $9.0 \mathrm{E}-09$ & $9.0 \mathrm{E}-10$ & $9.0 \mathrm{E}-11$ \\
& 5.0 & $1.5 \mathrm{E}-05$ & $2.2 \mathrm{E}-06$ & $2.2 \mathrm{E}-07$ & $2.2 \mathrm{E}-08$ & $2.2 \mathrm{E}-09$ & $2.2 \mathrm{E}-10$ \\
& 10.0 & $3.0 \mathrm{E}-05$ & $4.5 \mathrm{E}-06$ & $4.5 \mathrm{E}-07$ & $4.5 \mathrm{E}-08$ & $4.5 \mathrm{E}-09$ & $4.5 \mathrm{E}-10$ \\
& & & & & & & \\
Dichlorobenzene & & & & - & & & \\
& 0.5 & $1.3 \mathrm{E}-06$ & $2.0 \mathrm{E}-07$ & $2.0 \mathrm{E}-08$ & $2.0 \mathrm{E}-09$ & $2.0 \mathrm{E}-10$ & $2.0 \mathrm{E}-11$ \\
& 2.0 & $5.2 \mathrm{E}-06$ & $7.8 \mathrm{E}-07$ & $7.8 \mathrm{E}-08$ & $7.8 \mathrm{E}-09$ & $7.8 \mathrm{E}-10$ & $7.8 \mathrm{E}-11$ \\
& 5.0 & $1.3 \mathrm{E}-05$ & $2.0 \mathrm{E}-06$ & $2.0 \mathrm{E}-07$ & $2.0 \mathrm{E}-08$ & $2.0 \mathrm{E}-09$ & $2.0 \mathrm{E}-10$ \\
& 10.0 & $2.6 \mathrm{E}-05$ & $3.9 \mathrm{E}-06$ & $3.9 \mathrm{E}-07$ & $3.9 \mathrm{E}-08$ & $3.9 \mathrm{E}-09$ & $3.9 \mathrm{E}-10$ \\
& & & & & & & \\
\hline
\end{tabular}


TABLE 18:

POHC CONCENTRATION IN FLUE GAS

\section{FEEDSTOCK NO. 6}

\begin{tabular}{|c|c|c|c|c|c|c|c|}
\hline & \multirow[b]{2}{*}{$\begin{array}{l}\text { Wt. } \% \\
\text { In Feed }\end{array}$} & & \multicolumn{5}{|c|}{ FLUE GAS CONCENTRATION } \\
\hline & & & 0.990000 & 0.999000 & $\begin{array}{c}\text { DRE } \\
\underline{0.999900}\end{array}$ & $\underline{0.999990}$ & 0.999999 \\
\hline \multirow[t]{4}{*}{ Naphthalene } & 0.5 & $1.2 \mathrm{E}-06$ & $1.8 \mathrm{E}-07$ & $1.8 \mathrm{E}-08$ & $1.8 \mathrm{E}-09$ & $1.8 \mathrm{E}-10$ & $1.8 \mathrm{E}-11$ \\
\hline & 2.0 & 4.9E-06 & $7.4 \mathrm{E}-07$ & $7.4 \mathrm{E}-08$ & 7.4E-09 & $7.4 \mathrm{E}-10$ & $7.4 \mathrm{E}-11$ \\
\hline & 5.0 & $1.2 \mathrm{E}-05$ & $1.8 \mathrm{E}-06$ & $1.8 \mathrm{E}-07$ & $1.8 \mathrm{E}-08$ & $1.8 E-09$ & $1.8 \mathrm{E}-10$ \\
\hline & 10.0 & $2.5 E-05$ & 3.7E-06 & 3.7E-07 & 3.7E-08 & 3.7E-09 & 3.7E-10 \\
\hline \multirow[t]{4}{*}{ Dichlorobenzene } & 0.5 & $1.1 E-06$ & $1.6 \mathrm{E}-07$ & $1.6 \mathrm{E}-08$ & $1.6 \mathrm{E}-09$ & $1.6 \mathrm{E}-10$ & $1.6 \mathrm{E}-11$ \\
\hline & 2.0 & $4.3 \mathrm{E}-06$ & $6.4 \mathrm{E}-07$ & $6.4 \mathrm{E}-08$ & $6.4 \mathrm{E}-09$ & $6.4 \mathrm{E}-10$ & $6.4 \mathrm{E}-11$ \\
\hline & 5.0 & $1.1 \mathrm{E}-05$ & $1.6 \mathrm{E}-06$ & $1.6 \mathrm{E}-07$ & $1.6 \mathrm{E}-08$ & $1.6 \mathrm{E}-09$ & $1.6 \mathrm{E}-10$ \\
\hline & 10.0 & $2.2 E-05$ & 3.2E-06 & 3.2E-07 & 3.2E-08 & $3.2 \mathrm{E}-09$ & $3.2 \mathrm{E}-10$ \\
\hline
\end{tabular}


TABLE 19:

DETECTION LIMITS INDICATED BY RADIAN CORPORATION

\begin{tabular}{|c|c|c|}
\hline 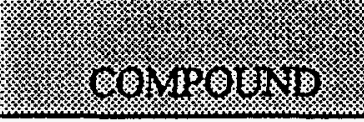 & (BETECTION InIm) & 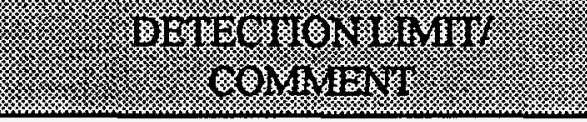 \\
\hline Vinylchloride & EPA Method $0030 \& 5040$ & $\begin{array}{l}\text { ( } 0.5 \mathrm{ng} / \mathrm{L} \text { for } 20 \mathrm{~L} \text { sample }) \\
\text { Validated method. Special pre- } \\
\text { cautions required to avoid sorbent } \\
\text { breakthrough. }\end{array}$ \\
\hline Toluene & EPA Method $0030 \& 5040$ & $\begin{array}{l}\text { ( } 0.5 \mathrm{ng} / \mathrm{L} \text { for } 20 \mathrm{~L} \text { sample) } \\
\text { Validated method. }\end{array}$ \\
\hline Tetrachloroethylene & EPA Method $0030 \& 5040$ & $\begin{array}{l}(0.5 \mathrm{ng} / \mathrm{L} \text { for } 20 \mathrm{~L} \text { sample }) \\
\text { Validated method. }\end{array}$ \\
\hline Ethylene Glycol & EPA Method $0010 \& 8270$ & $\begin{array}{l}\text { ( } 5 \mu \mathrm{g} / 100 \mathrm{scf} \text { sample) } \\
\text { Method requires validation. }\end{array}$ \\
\hline Phenol & EPA Method $0010 \& 8270$ & $\begin{array}{l}\text { ( } 5 \mu \mathrm{g} / 100 \mathrm{scf} \text { sample) } \\
\text { Method validated. }\end{array}$ \\
\hline Naphthalene & EPA Method $0010 \& 8270$ & $\begin{array}{l}\text { ( } 5 \mu \mathrm{g} / 100 \text { scf sample) } \\
\text { Target compound of method. }\end{array}$ \\
\hline 1,2-Dichlorobenzene & EPA Method $0010 \& 8270$ & $\begin{array}{l}5 \mu \mathrm{g} / 100 \text { scf sample) } \\
\text { Low recovery reported from volatile } \\
\text { loss in sample prep step. }\end{array}$ \\
\hline
\end{tabular}

(Our experience is that it may be better to select compounds other than ethylene glycol and phenol for the demonstration, if feasible. These compounds can be difficult for experienced laboratories. Alternative compounds could be selected that enhance unit performance and project success.) 
For the seventh feedstock, a 60-day test period with 1,080 hours of actual run time is planned. The 1,080 hours of operation will include 96 hours of preheating and conditioning, 936 hours of surrogate feeding, and 48 hours of transition'cool-down. The test program will include a contimuous long churation test lasting 824 hours to verify operability, reliability, availability and maintainability.

Each of the operational tests will be brought to steady state in accordance with this plan. In the event of an emergency shutdown prior to sample acquisition, the test will be repeated after purging the system and restarting from a cleaned system. Sample acquisition will begin after two hours of feed time. By definition, once a set of samples has been collected, the test run is considered successful. In general for this Test Plan, the test shall be considered successful if:

1) Steady-state conditions are achieved for each subtest/test and sustained for at least four hours,

2) Representative samples of flue gas and solids are obtained,

3) Material and heat balances close within +1 to $5 \%$, and

4) POHC destruction and removal efficiency exceeds $99.99 \%$.

An independent laboratory - Centre Analytical Laboratories, Inc. in State College, Pennsylvania will witness and audit the preparation of the feedstock prior to the start of each test. This laboratory will also provide analysis of the test samples. 


\section{SECTION 2.0}

\section{TEST PROCEDURE}

\subsection{FEEDSTOCK PREPARATION}

Approximately 500 to 1,500 pounds of feedstock will be made up just before the start of each test. Feedstock preparation will be based on the procedures as indicated in the Surrogate Feedstock Identification List (Task 1.1). Preparation of the feedstock will be witnessed, audited, and certified by a staff member from the analytical service group. The heterogeneous nature of the various feedstocks makes it extremely difficult to sample and assay. The volatile nature of some of the organic constituents requires the feedstock to be prepared just before an operational test to assure that the constituents will be present in the actual feed to the steam reformer. The six feedstocks are as follows:
No. $\quad$ Feedstock
1) Heterogeneous Debris
2) Aqueous Halogenated Organic Liquids
3) Absorbed Aqueous Organic Liquids
4) High Organic Content Sludge
5) Cement, Sludge, Ash and Solids
6) Natural Aqueous Waste

Feedstock raw materials will be purchased from known suppliers and kept in unopened or closed containers until the feedstock is to be made up. Some of the organic components may be made up as a stock solution by the analytical service group prior to the start of a test run. The prepared feedstock is to be kept in a closed 55-gallon drum or appropriate container. To the extent possible, the drum is to be kept in a cool area and out of direct sunlight. A log sheet is to be prepared indicating date and time, final weight of the feedstock, weight, source, purity of the components, and the name and signature of the analytical service group representative. 
The proposed feedstock testing order is No. 6 , No. 2 , No. 4 , No. 1, No. 3 , and No. 5 . It is expected that laboratory analysis will be available within three weeks after the samples have been taken. Results of the initial testing will be used to revise conditions, as necessary, for the subsequent tests. The rationale for this order is twofold: Feedstock No. 6 is mostly an aqueous feed material with a minimum amount of solid residue (ash). Feedstock No. 2 is also easy to feed. This feedstock requires the lock-hopper feeder subsystem. The most difficult material to feed is Feedstock No. 5 which contains a high percentage of residual solids that must be removed from the first-stage steam reformer bed on an hourly basis. This selection of feedstock test order allows ThermoChem/MTCI to leam more about the actual hardware and individual equipment items, or process functions, one item or function at a time. Ground glass frit or glass ballotini (150 microns mean size) will be purchased and fed into the first-stage steam reformer along with the feedstock to simulate vitrification operation.

A seventh surrogate feedstock will also be considered. Ion exchange resins are included in each of the six surrogates in small quantities. Ion exchange resins as beads and powders represent a significant waste stream from both DOE and commercial sources. Resins tested separately would provide valuable information on yet another possible waste stream for the steam reformer technology. A screening test will be done on surrogate ion exchange resins in a smaller test unit and the results of this test and funding availability will determine if an extra surrogate test in the $90 \mathrm{lb}$ per hour will be conducted.

The sorbent pads (polypropylene) will be shredded and stored in 55-gallon drums. A mixture of mineral oil and monochlorobenzene in the proportions listed in Table 3 will be prepared. This mixture will be prepared on a daily basis and stored in 5-gallon buckets (for nominal composition) or 30-gallon drums (for HIGHI and HIGH2 compositions). The shredded pads will be metered through the screw feeder while the liquid mixture will be pumped and sprayed on to the shredded material via an injection port in the injection screw. The cerium compounds (cerium fluoride and cerium oxide) are immiscible with oil-monochlorobenzene mixture and also are insoluble in water. Therefore, additional imestigation is needed to prepare a uniformly blended feedstock. We plan to perform some bench-scale tests with the feedstock constituents to identify the most appropriate feed method for each of the components commensurate with uniformity of feed composition. We also propose to 
contact Sandia National Laboratory and Idaho National Engineering Laboratory staff to get their input in this matter.

The mercury compounds pose a feeding difficulty similar to that of the cerium conpounds. Additionally, they are extremely toxic and require stringent health and safety procedures in handling. Therefore, it is proposed to conduct additional investigations as in the case of cerium compounds to identify the appropriate procedures for feedstock preparation and injection into the steam reformer.

\subsection{START-UP AND OPERATIONAL PROCEDURES}

Prior to each start-up, the unit is to have a mechanical checkout to assure that all components are in working order and that a sufficient supply of compressed air and nitrogen purge gas is available to perform the test. The unit should be empty of the sand or glass frit bed.

\subsubsection{Start-Up}

The start-up of the PDU consists of the following steps:

1) Start air compressor.

2) Start boiler. When water starts to boil, start superheater. Steam should be vented at this step.

3) Start the I.D. fan.

4) Supply fluidizing air to pass through the system, including the first-stage steam reformer, the second-stage steam reformer, thermal oxidizer, spray dryer, HEPA filter, activated carbon filters, I.D. fan and stack.

5) Start feeding the bed material (sand or glass frit).

6) Turn on electric heater of the first-stage steam reformer and raise the temperature such that the thermocouple embedded in the refractory increases at the rate of $100^{\circ} \mathrm{F}$ per hour. When the temperature reaches the operating window of 1000 to $1200^{\circ} \mathrm{F}$, the thermostat will maintain the desired bed temperature in the range of 1000 to $1200^{\circ} \mathrm{F}$.

7) - Turn on the natural gas to the first-stage steam reformer, turn on the oxygen supply to the second-stage steam reformer, and air to the thermal oxidizer. This will raise temperatures of the second-stage steam reformer and thermal oxidizer. Maintain the 
temperature increase at a rate of $100^{\circ} \mathrm{F}$ per hour. When the temperature exceeds $2000^{\circ} \mathrm{F}$, manually reduce the gas supply to maintain the temperature at about $2000^{\circ} \mathrm{F}$. Supply a small amount of superheated steam, if necessary, to the first-stage steam reformer to regulate the temperatures in all the zones.

8) Monitor the temperature at the exit of the spray cooler. When the temperature reaches $250^{\circ} \mathrm{F}$, turn on the water pump of the soda ash/water feed system. The agitator tank should be filled by water (no soda ash) at this time.

9) When the temperature at the inlet to the HEPA filter reaches $250^{\circ} \mathrm{F}$, switch fluidizing medium from compressed air to superheated steam.

10) Maintain the overall system temperature profile during steam fluidization at design values for one hour. This will end the preheating process.

In the case of liquid feedstock, the feed system will consist of a Moyno positive displacement pump. For mainly dry feedstock, a screw feeder will be used. Both feeders will be installed inside a lock vessel. In the test with liquid feedstock, water will be fed at a certain rate depending on the water content in the feedstock. When the proper temperature profile is established, the real feedstock feed will start at $90 \mathrm{lb} / \mathrm{hr}$.

\subsubsection{Feed Subsvstem}

Concurrent with the start-up, the surrogate feedstock is to be made up and the composition validated by an independent analytical service. Prior to the start of a test, the lock-hopper feeder subsystem is to be recalibrated (see Section 5.1). The metering screw setting should be based on the proper setting for the feedstock. The lock hopper should be charged with 90 pounds of feed and the time recorded. Each hour after, another 90 pounds of feedstock is to be added to the lock hopper. Adjustments should be made to the metering screw setting (RPM) to maintain the required feed rate within acceptable tolerances. The log sheet should be filled out with recordings of the feedstock quantities each hour and setting of the metering screw.

In the case of the seventh feedstock (surrogate waste for Piketon simulation), the calibration will be performed at the nominal feed rate of 20 pounds per hour. 
When liquids are fed into the system (surrogate Feedstock No. 6), the solids feeder subsystem is to be disengaged after the sand bed has been added and prior to starting the preheat cycle. Liquid is fed into the bottom of the first-stage steam reformer at a controlled rate of $90 \mathrm{PPH}$ via a steam-heated injector tube that is located near the bottom of the first-stage steam reformer. The solids in the liquid feed should be limited to about 3.0 mesh as an upper size to avoid plugging. The feed tank is to sit on a platform scale and is connected to the metering pump via flexible hose. The initial charge of feedstock is to be weighted with weight readings taken as indicated on the log sheet. Additions of feedstock to the feed tank are to be recorded. A second aqueous make-up tank will be used to hold the entire charge of feedstock. Both the feed tank and the make-up tank can be steam. heated and both tanks have pumps to recirculate the tank contents. The aqueous make-up tank also has an agitator for use, if required. Depending on the feedstock, prior to completing an operational test, diluent may be added to flush out this subsystem. Once the diluent is added to the feed tank, the test is terminated and the operational mode is shifted toward a controlled shutdown.

\subsubsection{Test Matrix}

The proposed matrix for the six feeedstocks is presented in Table 20. Each test consists of a total of 8 to 20 hours feedstock feed time. For each feedstock, there are two separate tests. One consists of four subtests with different temperatures in the first- and second-stage steam reformers. The objective is to find the optimal operating conditions. The second test parameters will be chosen from the first test data with the objective to obtain the best destruction efficiency. Preheat time for each test ranges from 12 to 20 hours and cool-down or transition time will range between 2 to 12 hours. Tests are planned with and without the second-stage reformer on-line to evaluate its influence on DRE. The first-stage steam reformer temperature is kept low $\left(1000\right.$ to $\left.1200^{\circ} \mathrm{F}\right)$ to minimize vaporization of the radionuclide species.

The test matrix for the seventh feedstock is given in Table 21. The unit will be operational for about 1,080 hours with a total feed time of about 936 hours. Six different feedstock compositions (listed in Table 3) will be used. The first four tests are intended to examine the effect of bed temperature and oxygen concentration on performance and emissions, map out the operational boundary, and identify 
TABLE 20:

\section{TEST MATRIX OF SURROGATE TREATMENT}

TESTS FOR FEEDSTOCK OF NATURAL AQUEOUS WASTE (\#6)

\begin{tabular}{|c|c|c|c|c|c|}
\hline Test Number & & & & & 2 \\
\hline Feed Rate of Surrogate $(\mathrm{lb} / \mathrm{hr})$ & 90 & 90 & 90 & 90 & 90 \\
\hline Preheating Time (hrs) & 12 & 10 & 2 & 2 & 20 \\
\hline Surrogate Feeding Time (hrs) & 4 & 4 & 4 & 4 & 20 \\
\hline Cooldown/Transition Time (hrs) & 2 & 4 & 4 & 12 & 12 \\
\hline Temperature of 1st Steam Reformer $\left({ }^{\circ} \mathrm{F}\right)$ & 1000 & 1000 & 1100 & 1200 & optimum \\
\hline Temperature of 2 nd Steam Reformer $\left({ }^{\circ} \mathrm{F}\right)$ & - & 2000 & 2000 & 2000 & optimum \\
\hline
\end{tabular}

TESTS FOR FEEDSTOCK OF AQUEOUS HALOGENATED ORGANIC LIQUIDS (\#2)

\begin{tabular}{|l|c|c|c|c|c|}
\hline Test Number & \multicolumn{4}{|c|}{3} & 4 \\
\hline Feed Rate of Surrogate (lb/hr) & 90 & 90 & 90 & 90 & 90 \\
\hline Preheating Time (hrs) & 12 & 10 & 2 & 2 & 20 \\
\hline Surrogate Feeding Time (hrs) & 4 & 4 & 4 & 4 & 20 \\
\hline Cooldown Transition Time (hrs) & 2 & 4 & 4 & 12 & 12 \\
\hline Temperature of Ist Steam Reformer $\left({ }^{\circ} \mathrm{F}\right)$ & 1000 & 1000 & 1100 & 1200 & optimum \\
\hline Temperature of 2nd Steam Reformer $\left({ }^{\circ} \mathrm{F}\right)$ & - & 2000 & 2000 & 2000 & optimum \\
\hline
\end{tabular}

TESTS FOR FEEDSTOCK OF HIGH ORGANIC CONTENT SLUDGES (\#4)

\begin{tabular}{|c|c|c|c|c|c|}
\hline Test Number & \multicolumn{4}{|c|}{5} & 6 \\
\hline Feed Rate of Surrogate $(\mathrm{lb} / \mathrm{hr})$ & 90 & 90 & 90 & 90 & 90 \\
\hline Preheating Time (hrs) & 14 & 8 & 2 & 2 & 20 \\
\hline Surrogate Feeding Time (hrs) & 4 & 4 & 4 & 4 & 20 \\
\hline Cooldown/Transition Time (hrs) & 2 & 4 & 4 & 10 & 12 \\
\hline Temperature of 1st Steam Reformer $\left({ }^{\circ} \mathrm{F}\right)$ & 1200 & 1200 & 1100 & 1000 & optimum \\
\hline Temperature of 2nd Steam Reformer $\left({ }^{\circ} \mathrm{F}\right)$ & - & 2000 & 2000 & 2000 & optimum \\
\hline
\end{tabular}

TESTS FOR FEEDSTOCK OF HETEROGENEOUS DEBRIS (\#1)

\begin{tabular}{|l|c|c|c|}
\hline Test Number & \multicolumn{2}{|c|}{7} & 8 \\
\hline Feed Rate of Surrogate (b/hr) & 90 & 90 & 90 \\
\hline Preheating Time (hrs) & 14 & 9 & 20 \\
\hline Surrogate Feeding Time (hrs) & 4 & 4 & 16 \\
\hline Cooldown/Transition Time (hrs) & 2 & 11 & 10 \\
\hline Temperature of 1st Steam Reformer $\left({ }^{\circ} \mathrm{F}\right)$ & 1100 & 1100 & optimum \\
\hline Temperature of 2nd Steam Reformer $\left({ }^{\circ} \mathrm{F}\right)$ & - & 2000 & optimum \\
\hline
\end{tabular}


TABLE 20:

TEST MATRDX OF SURROGATE TREATMENT

(Cont'd)

TESTS FOR FEEDSTOCK OF ABSORBED AQUEOUS ORGANIC LIQUIDS (\#3)

\begin{tabular}{|l|c|c|c|}
\hline Test Number & \multicolumn{2}{|c|}{9} & 10 \\
\hline Feed Rate of Surrogate $(\mathrm{lb} / \mathrm{hr})$ & 90 & 90 & 90 \\
\hline Preheating Time (hrs) & 14 & 9 & 20 \\
\hline Surrogate Feeding Time (hrs) & 4 & 4 & 16 \\
\hline Cooldown/Transition Time (hrs) & 2 & 11 & 10 \\
\hline Temperature of 1st Steam Reformer $\left({ }^{\circ} \mathrm{F}\right)$ & 1100 & 1100 & optimum \\
\hline Temperature of 2nd Steam Reformer $\left({ }^{\circ} \mathrm{F}\right)$ & - & 2000 & optimum \\
\hline
\end{tabular}

TESTS FOR FEEDSTOCK OF CEMENT, SLUDGES, ASHES AND SOLIDS (\#5)

\begin{tabular}{|c|c|c|c|}
\hline Test Number & \multicolumn{2}{|c|}{11} & 12 \\
\hline Feed Rate of Surrogate $(\mathrm{lb} / \mathrm{hr})$ & 90 & 90 & 90 \\
\hline Preheating Time (hrs) & 14 & 9 & 20 \\
\hline Surrogate Feeding Time (hrs) & 4 & 4 & 16 \\
\hline Cooldown/Transition Time (hrs) & 2 & 11 & 10 \\
\hline Temperature of lst Steam Reformer $\left({ }^{\circ} \mathrm{F}\right)$ & 1100 & 1100 & optimum \\
\hline Temperature of 2 nd Steam Reformer $\left({ }^{\circ} \mathrm{F}\right)$ & - & 2000 & optimum \\
\hline
\end{tabular}

OPTIONAL TEST FOR ION EXCHANGE RESINS

\begin{tabular}{|l|c|c|}
\hline Test Number & \multicolumn{2}{|c|}{13} \\
\hline Feed Rate of Surrogate (lb/hr) & 90 & 90 \\
\hline Preheating Time (hrs) & 14 & 9 \\
\hline Surrogate Feeding Time (hrs) & 4 & 4 \\
\hline Cooldown/Transition Time (hrs) & 2 & 11 \\
\hline Temperature of 1st Steam Reformer ( $\left.{ }^{\circ} \mathrm{F}\right)$ & 1100 & 1100 \\
\hline Temperature of 2nd Steam Reformer ( $\left.{ }^{\circ} \mathrm{F}\right)$ & - & - \\
\hline
\end{tabular}

TOTAL OPERATING HOURS: 616

TOTAL FEED HOURS: 180 


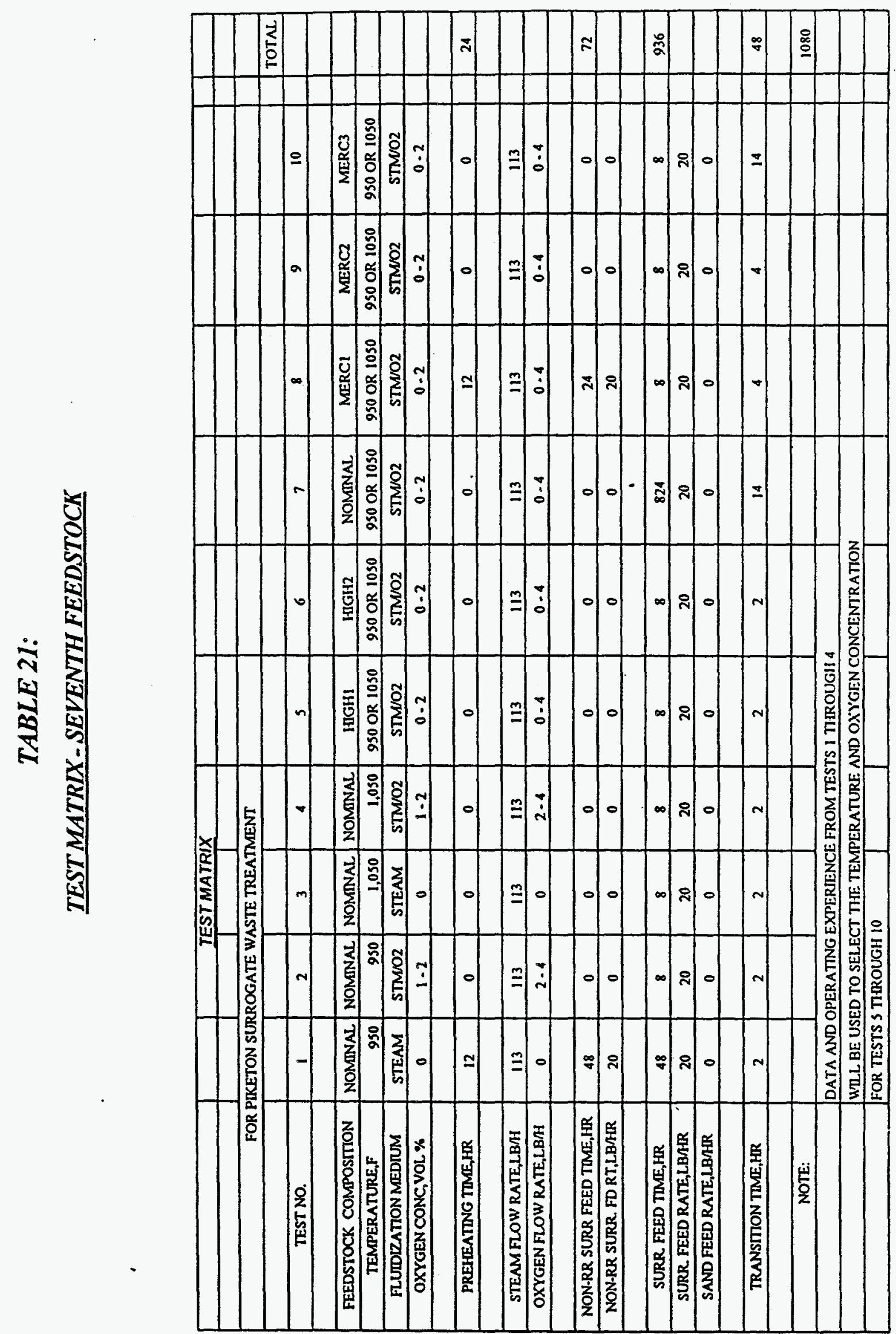


the optimum conditions for waste treatment. These optimum conditions will be employed in tests 5 through 10. Tests 5 and 6 employ high concentrations of monochlorobenzene and cerium compounds in order to demonstrate greater than $99.9999 \%$ DRE for the former and greater than $99.99 \%$ retention for the latter. Test 6 is a duplicate of Test 5 to demonstrate repeatability. Test 7 is a contimuous, long duration test to verify unit operability, reliability, availability and maintainability. Tests 8,9 and 10 are intended to evaluate the fate and capture of mercury compounds in the case of steam-reforming technology. Additional safeguards and health and safety procedures are required to perform these tests. Also, it is necessary to apply for and obtain an air permit from the Maryland Department of the Environment prior to this test run. The original permit covers only six feedstocks identified in Table 2. The addition of mercury to the seventh feedstock triggers environmental, health and safety concerns which need to be addressed prior to testing.

\section{Temperature Controls}

The indirect, controlled heating of the first-stage steam reformer requires precise temperature control. A series of thermocouples are imbedded in the steam reformer. These thermocouples provide the input to control the steam reformer temperature. In addition, both first- and second-stage reformers have a high-temperature cut-out that will cause an alarm to sound at a preset temperature, and if the high temperature is not corrected within an additional $50^{\circ} \mathrm{F}$, the necessary cut-out will shut off the feed supply and shut the unit down in a controlled manner.

The temperature of the superheated steam and the temperature of the gaseous effluent from the second-stage steam reformer are controlled. The fluidizing steam is at approximately 60 psig and $307^{\circ} \mathrm{F}$ as it enters the superheater. The function of the superheater is to heat the steam to as close to the first-stage steam reformer bed temperature as possible. Should the steam temperature not be as high as desired, the electric heater of the first-stage steam reformer of the unit will supply the necessary calorific input. These temperatures are monitored both on the supply side and the discharge side for each stream (4 readings).

The temperature profile for a given test program is established and the process controllers provide the control function to hold the temperature for both the first-stage and second-stage steam 
reformers. Results of on-line gas sampling and monitoring may be used to adjust the test temperature profile.

\section{Pressure Monitoring}

The steam-reforming system operates at or near atmospheric pressure. Pressure taps are located on each item of equipment and across the fluidized bed in the first-stage steam reformer and across the filter at the top of the first-stage steam reformer. System pressure measurements will be taken approximately every 15 minutes and recorded on the log sheet. If the pressure drop across the filter starts to increase, the filter can be cleaned by backflowing a small quantity of superheated steam into the first-stage steam reformer. The overall pressure of the first-stage steam reformer is monitored and if the pressure builds up, the operator will shut the system down and correct the problem.

Nitrogen gas is added at each pressure port. The total nitrogen flow is monitored and recorded on the log sheet. Gas concentration is measured based on the addition of the known quantity of nitrogen to the system.

\section{Process Control}

The surrogate treatment process will be monitored by temperature, pressure, and flow measurements along the whole system. Figure 3 shows the instrumentation diagram.

Continuous emissions monitoring will be performed downstream of the carbon filter or upstream of the I.D. fan. The on-line measurements will include $\mathrm{O}_{2}, \mathrm{CO}, \mathrm{CO}_{2}, \mathrm{THC}, \mathrm{NO}_{\mathrm{x}}$ and $\mathrm{SO}_{2}$. Gas and solids sampling will be performed on the different streams indicated in Figure 3. 


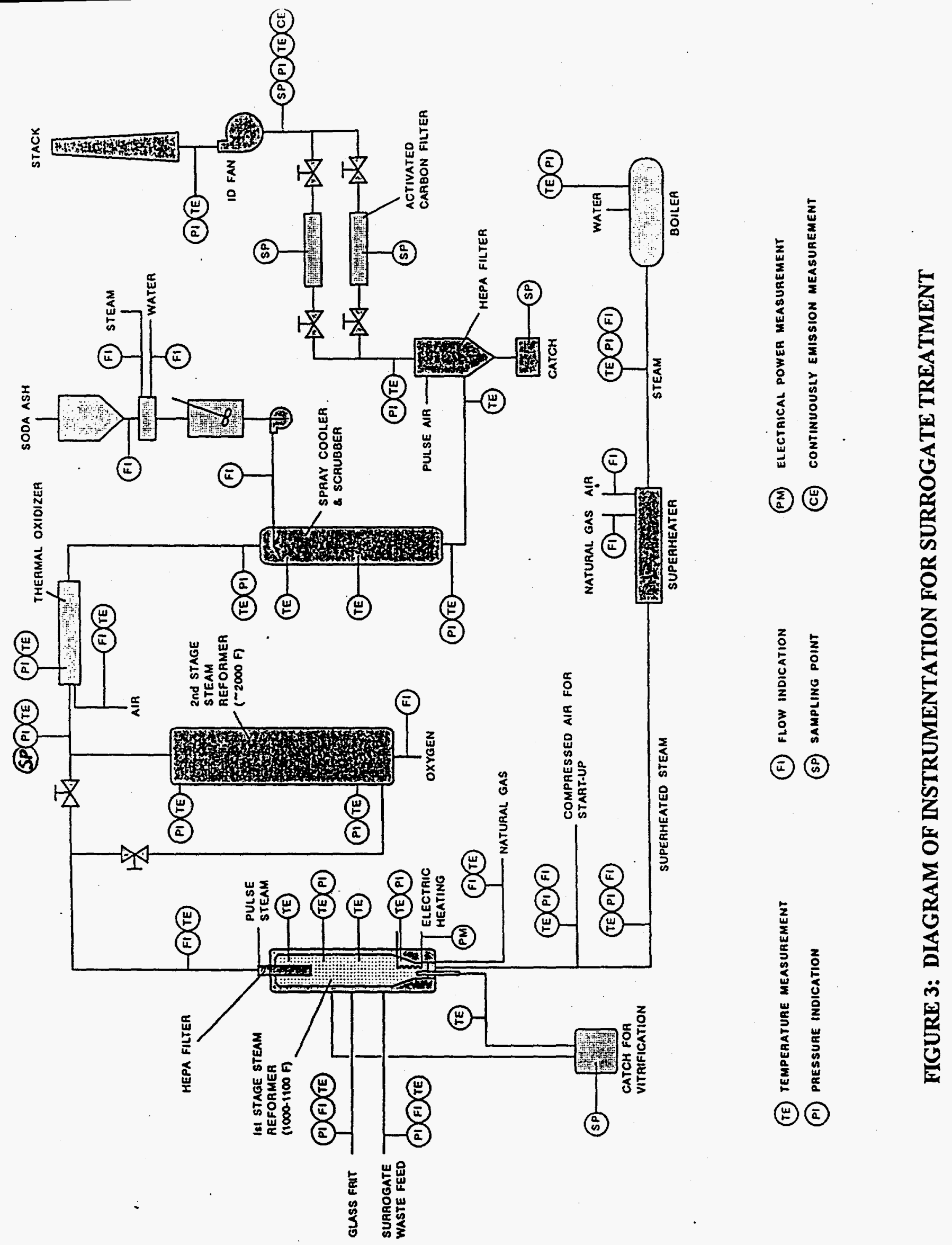


Process control will be provided by an on-line, continuous flue gas Horiba analyzer. Temperature profiles for both steam reformers are defined in the Text Matrix. The temperature before the HEPA filter will be controlled by a spray cooler/scrubber. According to the process conceptual design, the expected temperature profile should be as follows:

$\begin{array}{ll}\text { Inlet of the spray cooler } & 2000^{\circ} \mathrm{F} \\ \text { Inlet of the HEPA filter } & 250^{\circ} \mathrm{F} \\ \text { Inlet of the activated carbon filter } & 215-235^{\circ} \mathrm{F} \\ \text { Inlet of the I.D. fan } & 210-230^{\circ} \mathrm{F}\end{array}$

\section{Bed Removal}

Tests will require hourly removal of bed material during the 8- to 20-hour system feed schedule. Bed removal will be accomplished just after the lock-hopper feeder subsystem has been charged. The first-stage steam reformer operates at a slight pressure; the solids will be discharged via a valved opening to a vented container under a slight pressure. The container will be purged with nitrogen prior to the transfer. The container will be weighed prior to charging and after filling; both weights will be recorded on the log sheet. The container will be cooled by placing it in a pan of water, if necessary. The estimated initial temperature of the container will be less than $1000^{\circ} \mathrm{F}$ after discharging the bed.

Estimated removal rates for each feedstock are as follows:

\begin{tabular}{cr} 
Feedstock Number & Hourly Removal R \\
\hline 1 & $91-92$ \\
2 & $43-46$ \\
3 & $90-91$ \\
4 & $56-59$ \\
5 & $91-92$ \\
6 & 16 \\
7 & $4-5$
\end{tabular}


Actual bed discharge weights may vary and are approximate. Removal weights will be adjusted after each discharge to keep the bed depth at the required level. The removed bed material will be transferred to 55-gallon drums and saved until the end of the test run. Samples will be taken based on results of the test program. The final bed sample will be taken when the test has been completed and the bed has been removed. Bed material is to be removed during the shutdown operation based on a rate of approximately $100 \mathrm{PPH}$. The same removal procedure applies. The pressure drop across the fluidized bed is to be recorded.

\subsubsection{Normal Shutdown Procedures}

At the end of a test, the unit is to be shutdown in an organized, controlled manner. System shutdown starts after an 8 to 20 hour operational test or can be delayed if excess feedstock is available. For Fedstocks No. 1 to No. 5, where the feedstock is a solid, the shutdown starts 30 minutes after the last feedstock has been fed to the first-stage steam reformer (lock hopper is empty). For Feedstock No. 6, the shutdown starts once the feed tank has been flushed with water and the water injected into the first-stage steam reformer.

For feedstock 7, the shutdown starts once the monochlorobenzene and oil feed tank has been flushed with water and the water injected into the first-stage reformer.

The first equipment item to be shutdown is the thermal oxidizer. This should take place approximately 30 minutes after the lock hopper is empty.

Bed material is to be removed as indicated in Section 2.2.3. Fluidizing steam flow is to be stopped after the bed has been removed. Not all of bed may be removed by draining. Any remaining bed material is to be removed after the unit has cooled and shutdown is complete.

The temperature profile of the second-stage steam reformer is reduced at a rate of approximately $200^{\circ} \mathrm{F}$ per hour, the temperature profile for the first-stage steam reformer is reduced at a rate of approximately $100^{\circ} \mathrm{F}$ per hour. 
If it is believed that a sufficient quantity of bed material remains in the first-stage steam reformer after the bed material should have been removed (about 5 hours into the shutdown mode), nitrogen may be used in place of steam to assist in the shutdown procedure. In all cases, the steam flow is to be stopped once the temperature of the first-stage steam reformer is at $400^{\circ} \mathrm{F}$. Nitrogen purge gas is not required when the temperature of the first-stage steam reformer is $400^{\circ} \mathrm{F}$ or below. The shutdown conditions are to be recorded on the log sheet until ambient conditions are reached.

After the unit has cooled to ambient conditions, the unit is to be disassembled and cleaned. If there is residual bed material, it is to be weighed and saved. Any particulate on the filters is to be removed and saved. The filters are to be cleaned, inspected and reinstalled. The residual sorbent in the feed tank and spray cooler is to be removed and weighed and sampled. The units are to be recharged and reassembled. The thermal oxidizer does not require an inspection. It is to be serviced and made ready for the next test. 


\section{SECTION 3.0}

\section{EMERGENCY SHUTDOWN}

Two conditions are believed to potentially cause an emergency shutdown. The conditions are an over-pressure of the first-stage steam reformer and an overheating of either the first-stage or second-stage steam reformer units. An over-pressure could be caused by the first-stage filters becoming plugged. An overheating could be caused by the in-bed thermocouples being fouled. The shutdown procedure is slightly different for each case.

For all unplanned shutdowns, the feedstock supply will be secured. Depending on the quantity of feedstock and type of feedstock, a decision will be made as to the disposition of the material. Most likely, the feedstock will be saved and used for a final run to dispose of waste material or to provide an extended duration test run.

\subsection{OVER-PRESSURE EMERGENCY SHUTDOWN}

The first-stage steam reformer will be operating at a pressure slightly above atmospheric pressure, probably less than 5 psig. The system does not have pressure transmitters; a gauge with an adjustable alarm will sound at about $7.5 \mathrm{psig}$ and the alarm will sound again at $10 \mathrm{psig}$. At $7.5 \mathrm{psig}$, the filters will be back-flushed with nitrogen. If this does not solve the problem, the unit will be shutdown as a normal mode of operation. If the pressure does not decrease and the pressure reaches $10 \mathrm{psig}$, the emergency shutdown procedure will be in effect. The lock-hopper feeder subsystem will be stopped and the fluidizing steam flow rate will be reduced until the pressure drops to about 7.5 psig. If the pressure can be maintained at about $7.5 \mathrm{psig}$, the operational test will continue. Operator judgment will be used to evaluate the conditions if the pressure remains between 7.5 and 10 psig. If the pressure remains at the elevated level, the system will follow the normal shutdown procedure as to temperature reduction (heaters are turned off). In addition, as much bed material is to be removed as quickly as possible. If the pressure remains at $10 \mathrm{psig}$ or does not drop to $7.5 \mathrm{psig}$, the sparger steam flow is to be shut off. . 
An over-pressure in the second-stage steam reformer is not anticipated and is highly unlikely.

\subsection{OVER-TEMPERATURE EMERGENCY SHUTDOWN}

The electric heating elements for the first-stage steam-reforming unit are controlled by four thermocouples. The thermocouples' readings are averaged and the steam reformer has its own controller. The average temperature is also indicated and recorded on a computer. The heaters are either

on or off. Each bank of heaters work as a unit. A separate thermocouple connected to the surface of the steam reformer shell is a high-temperature readout on the computer system. These units are connected to an alarm which indicates an over-temperature of the shell. If the condition that caused the over-temperature is not corrected and the temperature continues to rise, the temperature switch will activate a relay that will shut off power to the heaters. The system is then shutdown using the normal procedures.

\subsection{POWER FAILURE}

In the unlikely even of a power failure, the whole system shuts down. No steam, no natural gas, no feed, no heat, and no thermal oxidizer. The nitrogen supply is a passive system and relies on gas cylinder pressure. All subsystems are secured and the nitrogen is added to the steam sparge system. The fluidized bed in the first-stage steam reformer is likely to have slumped and may be difficult to refluidize. The test run will be terminated and rescheduled if the power failure occurs before the first set of samples has been taken.

Power failures are infrequent in Baltimore, Maryland and have an occurrence of less than once per year. 


\subsection{MECHANICAL EQUTPMENT FAILURE \\ (non-emergency potential system shutdowns)}

Several types of mechanical failures have occurred over the past few years while operating the PDU. The mechanical failures have been limited to problems with the lock-hopper feeder subsystem, the liquid feed subsystem, and the steam supply. The mechanical problems are generally of a minor nature, and the feed may be off for a few minutes. If the feed subsystem can be restarted within a few minutes, the test will be continued. If the feed subsystem can not be restarted, the test will be terminated and the system will be shutdown as per standard shutdown procedures. If the system can not be restarted and the first set of samples has not been taken, the operation will be considered a nontest. 


\section{SECTION 4.0}

\section{STEAM REFORMER PERFORMANCE}

\subsection{OBJECTIVES}

The steam-reforming tests with the surrogate feedstocks as planned, provide information to evaluate: 1) the application of this technology on a scale that will provide reproducible results at a reasonable system capacity while meeting RCRA standards; 2) engineering aspects for system scale-up, system reliability, and operational costs; and 3) provides direction. for future development and applications. The test program may also address capacity considerations as each of the feedstocks may have different treatment rates, either above or below the design basis of $90 \mathrm{PPH}$ while meeting desired destruction efficiencies.

\subsection{HISTORY}

Two-stage steam reforming system was first considered by MTCI while conducting tests for Sandia National Laboratories on low-level mixed waste surrogate feedstock in early 1995 . This program used an existing refractory-lined unit, operating at a throughput of approximately $30 \mathrm{PPH}$ and a temperature of near $1000^{\circ} \mathrm{F}$. A slip stream of intermediate product gas was then treated in the benchscale unit. The results of this limited test program indicated that the second-stage unit would provide additional destruction efficiency and the gaseous effluent would meet free-release criteria. This initial activity has indicated the potential for treating the surrogate waste feedstock in this program.

\subsection{CONFIGURATION}

The steam-reforming system as configured will not have an aqueous discharge. This configuration will dry scrub acid gases which will also result in a solid waste. The test program will allow an assessment of the effectiveness of the dry absorption system as well.

Each of the surrogate feedstocks contain RCRA metals that should remain with the first-stage steam reformer bed residue. The bed residue will be assayed only for material balance information. Testing of the solid residue is not part of the Test Plan. 


\subsection{EXPECTATIONS}

The steam-reforming system as configured will have as a final stage a thermal oxidizer. The purpose of the thermal oxidizer is to convert carbon monoxide, ethylene, hydrogen, and methane into carbon dioxide and water vapor. A key to the successful conversion of long-chain refractory hydrocarbons at an elevated temperature is the presence of hydrogen gas. The endothermic reaction in the first-stage steam reformer should produce sufficient, excess hydrogen to drive the reactions to completion in the second-stage steam reformer, i.e., the breakdown of halogenated hydrocarbons to hydrogen chloride and to shorter chain hydrocarbons. 


\section{SECTION 5.0}

\section{CALIBRATION}

\subsection{LOCK-HOPPER FEEDER SUBSYSTEM}

The lock-hopper feeder subsystem consists of a feed screw, metering screws, and a lock hopper with a working volume of approximately 3 cubic feet. The design base capacity is estimated at 180 pounds. The feed screw operates at a constant RPM and the metering screws have a variablespeed drive. Prior to starting a test, the lock hopper feeder subsystem will be calibrated using a feed material with about the same consistency as the feedstock, i.e., density and particle size. Feed will be metered out of the lock hopper at three to five different metering screw (settings) RPMs. The discharge material will be weighed at each feeder setting (RPM) and a curve developed. The expected discharge rate of $90 \mathrm{PPH}$ will be in about the midpoint of the selected setting. Prior history has indicated that the lock-hopper feeder subsystem delivers less feed at the desired RPM when the unit is operating. This is due to back pressure in the steam reformer. The feed rate may even change during an operational test. The lock hopper will be charged once per hour, inspection during charging and actual of the first-stage reformer will provide a periodic indication of the feed rate. It is also expected that during any one-hour period, the instantaneous feed rate accuracy could be within a range of \pm 50 percent, the average feed rate should be within acceptable range of \pm 10 percent. The bed retention time of approximately one hour (bed discharge intervals) will also help in leveling out operating conditions.

The modified feed system for the seventh feedstock has a hopper capacity of about 10 cubic feet. This system has also variable-speed drive motors. The feed system will be calibrated with the shredded material for feed rates ranging from 5 to $35 \mathrm{lb} / \mathrm{hr}$. The measured rates will be checked against those computed from the loss-in-weight readings.

\subsection{LIQUID FEED SYSTEM}

The liquid feed subsystem consists of a feed make-up tank, feed tank, both with recirculating pumps, and a metering pump with a variable-speed drive. The feed tank will sit on a scale and the 
metering pump is on a skid. The tank will be filled with a liquid with a density and consistency similar to the feedstock. The metering pump will be set at three to five different settings and the volume of discharge will be collected and weighed. The scale setting will be recorded at the start and end of each calibration check. The two weight readings for each pump setting will be reconciled and a calibration curve will be developed. The feed rate will be validated by scale reading differences during the test program. Historically, the liquid feed rate is consistent providing the feedstock is uniform.

The liquid feed subsystem will be calibrated separately for feeding the liquid portion of feedstock 7 into the injection screw port. The calibration range will span 0 to $8.75 \mathrm{lb} / \mathrm{hr}$.

\subsection{TEMPERATURE ELEMENT CALIBRATION}

The temperature elements have a read-out in milliampere which is converted to a temperature reading in degrees Fahrenheit $\left({ }^{\circ} \mathrm{F}\right)$. The heating elements are controlled by averaging four temperature readings in the first-stage of steam reforming. The average temperature in the first-stage steam reformer sends a signal to both the temperature controller and the computer monitoring system. The temperature controller is a standard solid-state device that turns the heaters on and off to control the temperature at a preset point. The heaters are external to the bed and are well-insulated; the heating system has the ability to heat up rapidly and to cool down slowly. A separate temperature element and temperature indicator has the ability to override the temperature controllers and shut the system down if the temperature reaches a preset high temperature.

\subsection{PRESSURE INDICATORS}

Pressure indicators are standard gauges, either straight pressure or differential pressure. Prior to the start of the test program, the gauges will be calibrated against a known standard at a pressure of between 1 and 15 psig. The differential pressure gauges will be calibrated at a pressure differential of between 0 and 80 inches of water. After six operational tests, the gauges will be recalibrated. Except for the possible over-pressure of the first-stage steam reformer, pressure is not a significant variable. The first-stage steam reformer will operate at slightly above atmospheric pressure and the rest of the system at below atmospheric pressure. 


\section{SECTION 6.0}

\section{SAMPLING .}

Analytical procedures will be according to the standards accepted by the Environmental Protection Agency (EPA), such as:

$\begin{array}{lc}\text { Volatile Organic Compounds } & \text { EPA } 8260 \text { (liquids) } \\ \text { Volatile Organic Compounds } & \text { EPA TO-14 (gases - ppm level) } \\ \text { Semi Volatile Organic Compounds } & \text { EPA } 8270 \text { (liquids) } \\ \text { Trace Metals by Inductively Coupled Plasma } & - \\ \text { Ultimate Analysis Spectrometry } & \end{array}$

\subsection{GAS SAMPLING}

Gas samples will be taken either upstream of the thermal oxidizer or upstream of the I.D. fan. During the subtests such as $1,3,5,7,9$ and 11 , gas samples will be taken upstream of the thermal oxidizer to evaluate the influence of first-stage steam reformer temperature and/or second-stage steam reforming on destruction effectiveness. A gas sample - about 10 liters - will pass through two charcoal tubes in series, vacuum pump and flow meter. The second charcoal tube is used to estimate the accuracy of the sample caught by the first tube. The charcoal tubes with samples will be sent for analysis to the Centre Analytical Laboratories, Inc. in State College, Pennsylvania. A separate sample will also be taken during each subtest to generate a condensate for detecting the presence of surrogate radionuclide. Gas sampling and analysis for Tests 2, 4, 6, 8, 10 and 12 will be performed upstream of the I.D. fan by Radian Corporation in Austin, Texas, using the methods indicated in Table 19.

For the tests with the seventh feedstock, TRC Environmental Corporation will provide gas sampling and analysis services. The product gas will be sampled downstream of the second high-temperature HEPA filter/upstream of the thermal oxidizer. Stack flue gas sample will also be collected. The components to be monitored are: steam reformer off-gas composition $\left(H_{2}\right.$, 
$\mathrm{CO}, \mathrm{C}_{x} \mathrm{H}_{\mu}, \mathrm{CO}_{2}, \mathrm{HCl}, \mathrm{H}, \mathrm{S}, V O C, \mathrm{SVOC}, \mathrm{RCRA}$ metals and radiomuclides) and stack gas composition ( $\mathrm{O}_{2,} \mathrm{CO}, \mathrm{CO}_{2}, \mathrm{SO}_{2}, \mathrm{HCl}, \mathrm{NO}_{x}, \mathrm{C}_{x} \mathrm{H}_{\mathrm{p}}$ VOC, $\mathrm{SVOC}, \mathrm{RCRA}$ metals and radionuclides). Six samples at each location are planned during the non-mercury doped sorbent run and three samples at each location are planmed during the mercury-doped sorbent run. The latter will include one sample each during elemental mercury, reduced form mercury and oxidized form mercury doped sorbent runs. Dioxin and furan measurements are included. The stack gas will be monitored continuously on-line for $\mathrm{O}_{2}, \mathrm{CO}, \mathrm{SO}_{2}, \mathrm{NO}_{x}$, and $\mathrm{C}_{x} \mathrm{H}_{y}$

\subsection{SOLIDS SAMPLING}

Solid samples will be taken from:

1) Catch of first steam reformer,

2) Catch of HEPA filter after spray cooler, and

3) Activated carbon from activated carbon filter.

The samples will be taken in the middle of steady-state condition in each test/subtest. Representative, composite samples of each stream will be sent to outside laboratories for analysis of RCRA metals, radionuclide surrogates, TOC, TOX, total chloride and ash constituents.

For the seventh feedstock, the solids will be characterized as follows.

At the start of the test, initial representative samples of surrogate waste, bed material and carbon filter material will be taken. At the end of the test, final representative samples of these streams will be taken. These along with the different catch material will be used to make composite samples of the following: feedstock, sand, bed drain catch, HT HEPA1 catch, HT.HEPA2 catch, spray dryer catch, baghouse catch, and LTHEPA catch. The solid samples will be collected by ThermoChemMTCI. The samples will be sent to Centre Analytical Laboratory, State College, Pennsylvania for analysis. The analyses will include ultimate analysis, size analysis, RCRA metals, organics, and radiomuclides. Toxic Characteristic Leachate Procedure (TCLP) will be performed on the spent sorbent catch and the final carbon filter samples. 


\section{SECTION 7.0}

\section{IDENTIFICATION, ASSESSMENT, AND \\ QUANTIFICATION OF SOURCES OF ERROR}

\subsection{BED MATERIAL AND SOLID RESIDUE}

The heterogeneous nature of the feedstock can result in a non-uniform feed to the first-stage steam reformer. The feedstock will be prepared according to the instructions of WMIP 16. The percentage of RCRA metals and radionuclide surrogates is less than 1.5 percent of the feedstock as chemical compounds such as chromium chloride with 6 waters of hydration $(19.4 \% \mathrm{Cr})$. Any inaccuracy during either mixing or feeding could result in a period of time without any of a specific chemical compound being fed into the first-stage steam reformer. However, over the 8- to 20-hour test period, all of the chemical compounds will have been fed into the system. The bed material will be removed hourly, composited every two hours, and sampled every four hours (four bed samples per test). The filters will be checked and cleaned after the test and the particulate sampled and assayed or just assayed if the amount is insignificant. The closure on the weight should be within \pm 5 percent. Closure on RCRA metals is also expected to be within 10 percent. Closure of the RCRA metals and radionuclide metals of \pm 2 percent would be considered successful.

Solid residue is expected to be fairly uniform and consistent, except for feedstock containing vermiculite and perlite. The residue is not expected to contain any volatile organic compounds.

\subsection{CHLORINE BALANCE}

Closure on the chlorine balance is expected to have a broader accuracy range than that for the RCRA metals. The amount of chlorine being fed into the system can be accurately determined to about 5 pounds in 500 pounds of feed. Chlorine is expected to react with the sorbent soda ash and chlorine may escape with the process effluent. The dry weight of the sorbents can be determined and the percent chlorine can also be determined. The sorbents will be weighed and sampled once each operational test. The concentrations of chlorine in the effluent gas can also be determined but quantity determination is less precise. The effluent gas sample is scrubbed; both water vapor and hydrogen 
chloride are condensed. The condensate is weighed and the quantity of chlorine in the gas is a function of several measurements and one or two assays. Four effluent gas samples are expected for each test. The closure on the chlorine balance should be within \pm 1 percent. Detectable levels of chlorine are not expected in the effluent gas from the thermal oxidizer. Closure of the chlorine balance within \pm 10 percent would be considered successful.

\subsection{ORGANICS AND RCRA ORGANIC}

The feedstock is made up of a number of organic components such as activated carbon which is expected to report to the bed material; organic debris solids such as PVC. and.neoprene; and toluene, trichloroethylene, and mineral oil which are non-RCRA organics; and naphthalene, chlorobenzene, and 1,2-dichlorobenzene which are surrogate feedstock for RCRA compounds. Carbon is in the feedstock as a solid (PVC) and as a liquid (naphthalene). On a warm day, the low boiling point organic liquids may also exist in the feedstock as near vapor. The carbon content of the feedstock is expected to be assessed at or near 10 pounds of the 500 pounds of feedstock. The activated carbon is expected to report to the bed; the carbon in the bed material should have a closure of within \pm 5 percent. The remaining carbon should be as methane, carbon monoxide, ethylene, carbon dioxide ahead of the thermal oxidizer and carbon dioxide after the thermal oxidizer.

Prior test work by MTCI indicated a destruction and removal efficiency of greater than 99.99 percent for the RCRA organic surrogates using an existing single-stage steam reformer and treating a slip stream of product gas. With the revised configuration, these preliminary results are expected to be improved by two orders of magnitude.

Based on the made-up quantity of RCRA organic in the feedstock, the measured quantity of the RCRA organic in the effluent gas is expected to be less than 0.001 percent of the input with a target of 0.0001 percent. Depending on the feedstock, the RCRA organic content can range from a high of over 10 percent to a low of 0.5 percent. 
Closure of the carbon balance within an accuracy range of \pm 10 percent will be considered successful.

Destruction and Removal Efficiency (DRE) and Destruction Efficiencies (DE) will be based on computations of what was fed to the system and what is reported in the effluent gas on a mass basis.

Products of incomplete reaction are not anticipated. If initial test results indicate a potential deficiency, the feed rate to the first-stage steam reformer will be reduced if this appears to be a feasible solution or the thermal oxidizer conditions adjusted. 


\section{SECTION 8.0 \\ RESIDUAL WASTE DISPOSAL}

All of the residual waste will be saved until the end of the last test. All remaining feedstock that was not processed will be processed at a rate that is acceptable for achieving complete destruction of the RCRA organic components. A special feedstock may be prepared to provide a long duration test to indicate the reliability of the system. This waste feedstock probably will not include RCRA metals or organics or radionuclide surrogates.

The residual waste residue will be drummed and shipped as non-hazardous waste to a landfill. 


\section{SECTION 9.0}

\section{VITRIFICATION TESTS}

The solids drained from the first-stage steam reformer during Tests $2,4,6,8,10$ and 12 will be split into two parts - a small part used as sample for chemical analysis and material balance, and the major part inerted out with nitrogen gas and stored in closed containers for vitrification testing. These solids will be shipped to the Clemson University Vitrification Research Laboratory (CVRL), Clemson, South Carolina. CVRL will conduct crucible studies on each sample (minimum of six) to determine the glass characteristics and waste loadings using the as-received frit. To improve glass characteristics (waste loading, viscosity, leachability, hardness, etc.), minor modifications will be made, as needed, to glass frit content and tests will then be conducted in an Envitco PDU glass melter. For each of the glass samples produced, an analysis will be performed to determine waste loading, off-gas composition, and TCLP testing. The objective is to generate data on the vitrification characteristics (rate, ease of glass making, off-gas generation; and properties of glass) of the steam reformer downflow solids. 


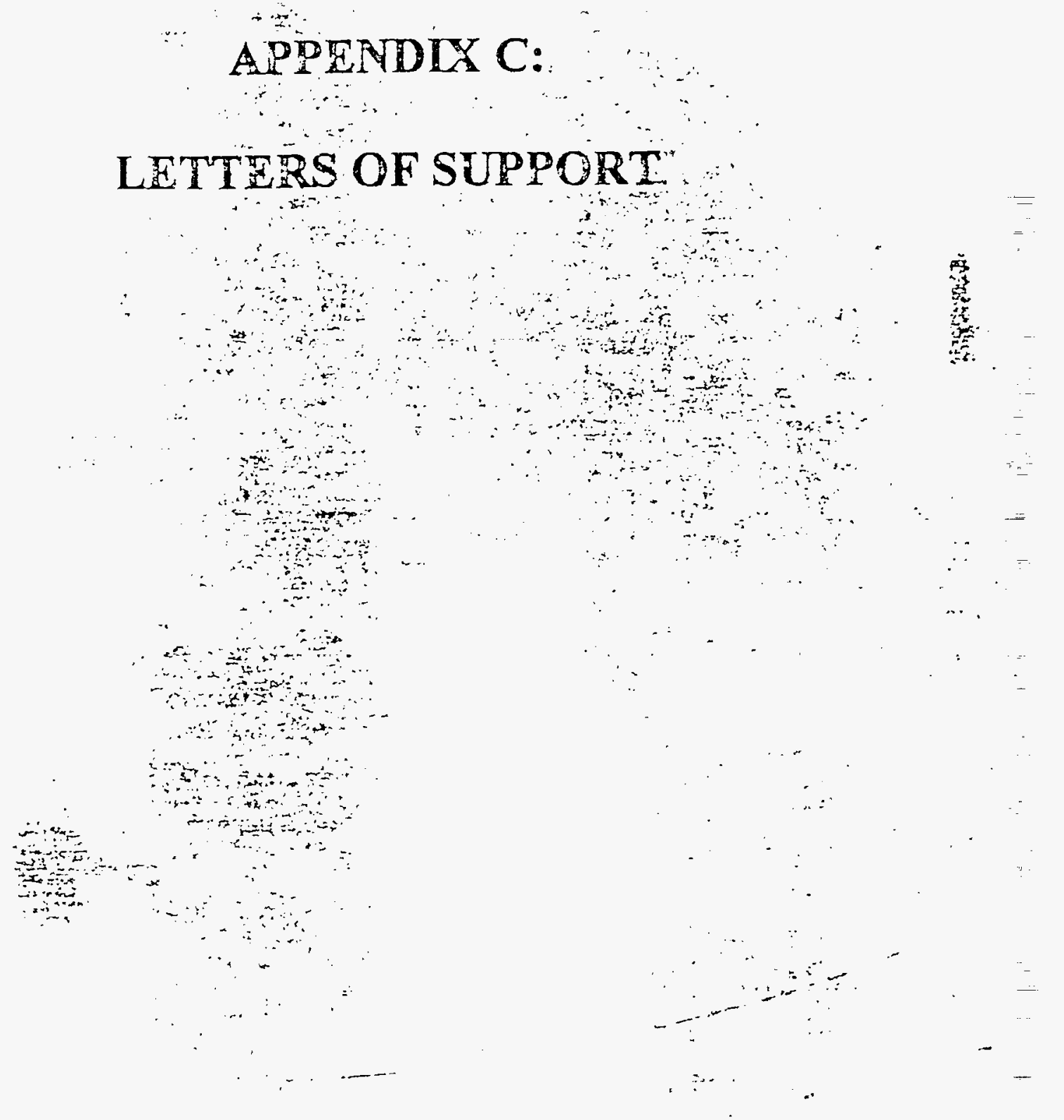


Oak Ridge Operations Office

P.O. Box 2001

Oak Ridge, Tennessee 37831-

May 8, 1997

Ms. Julie Cominer

U. S. Department of Energy

Idaho Operations Office

850 Energy Drive

MS 1225

Idaho Falls, Idaho $83401-1563$

Dear Ms. Conner:

\section{TECENOLOGY DEPLOYMISNT INITLATIVE}

Oak Ridge Operations Office (ORO) is pleased to offer the following proposal, Steam

Reformation of TSCA and Low-Level Mixed Waste, as a candidate technology to be used in the cleanup of U. S. Department of Energy (DOE) sites. This subroittal is in response to the call from DOE's Office of Science and Technology for Technology Deployment Initiative proposals.

The proposed low-level mixed waste (LIMW) steam reforming system will provide total destruction of hazardous halogensted organics, very significant levels of volume reduction, and concentration of toxic inorganic and radioactive cormponents in a non-leachable and stabilized final waste form. The overall system offers an environmentally safe, non-incinerating, cost-

is effective, and publicly acceptable method of processing LLMW.

If selected, you may expect a high level of commitment on the part of ORO to ensure the success of the project. Questions about this proposal may be addressed to Jolmny O. Moore at (423) 576-3536.

Sincerely,

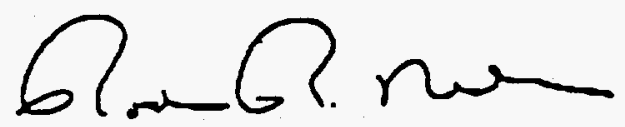

Rodney R. Nelson

Assistant Manager for

Environmental Management

Enclosure (1) 
DATE:

January 17,1997

KEPLYTO ATTN OF:

EF-21:Rafferty

SUBEECT:

TO:

\title{
PORTS SITE SUPPORT FOR A TREATABILITY STUDY USING THERMOCHEM'S STEAM REFORMING PROCESS
}

\author{
Clifford P. Carpenter, Morgantown Energy Technology Center, METC
}

The Portsmouth Gaseous Diffusion Plant began operation in 1954 to.enrich uranium for national defense and commercial reactors. Throughout the operating history of the plant over 100,000 cu. $f t$. of solid radioactively contaminated PCB waste has been generated. Treatment andlor uttimate disposal of this waste stream in accordance with applicable environmental regulations as well as DOE Orders is proving to be very challenging.

Over the past several months. we have been discussing the applicability of using steam reformation as a method of treating the PCB/Rad waste with Gary Voelker, Chief Operating Officer, of ThermoChem, Inc. Preliminary data suggests that steam reforming in conjunction with off gas treatment may be an effective method for treating mixed waste streams. In addition to the PCB/Rad waste, we have several other waste streams including spent activated carbon and ion exchange resin that may be treatable using steam reformation.

Identifying, testing and implementing innovative technologies will be required in order to meet the expectations identified in the Ten Year Plan. Although there is currenty no EM40 budget available at PORTS to support a technology demonstration involving steam reforming, we strongly believe that such a demonstration would be worthwhile. If EM-50 financial support could be provided, PORTS would be very interested in hosting this technology. PORTS has successfulby hosted many technology demonstrations and with the various waste streams, the existing infrastructure, the cooperattve attlude with the regulator community, the history of successfully moving from the demonstration stage to remediation of an existing unit, and the available technical expertise, PORTS is an ideal site for demonstrating steam reforming technology.

If you wish to discuss this issue in further detail, please contact Melda Rafferty of my staff at (614) 897-5521.

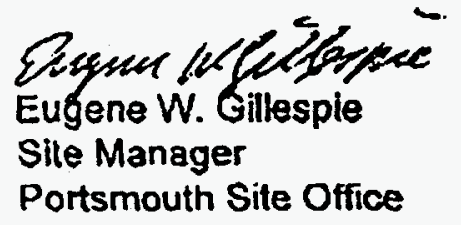




\section{APPENDCX D:}

\section{OPERATING PARAMETER TRENDS}

- Multiple Surrogate Tests, and

- Piketon Waste Simulation Tests

Q Parte Simulation Tests 
MULTIPLE SURROGATE TESTS 


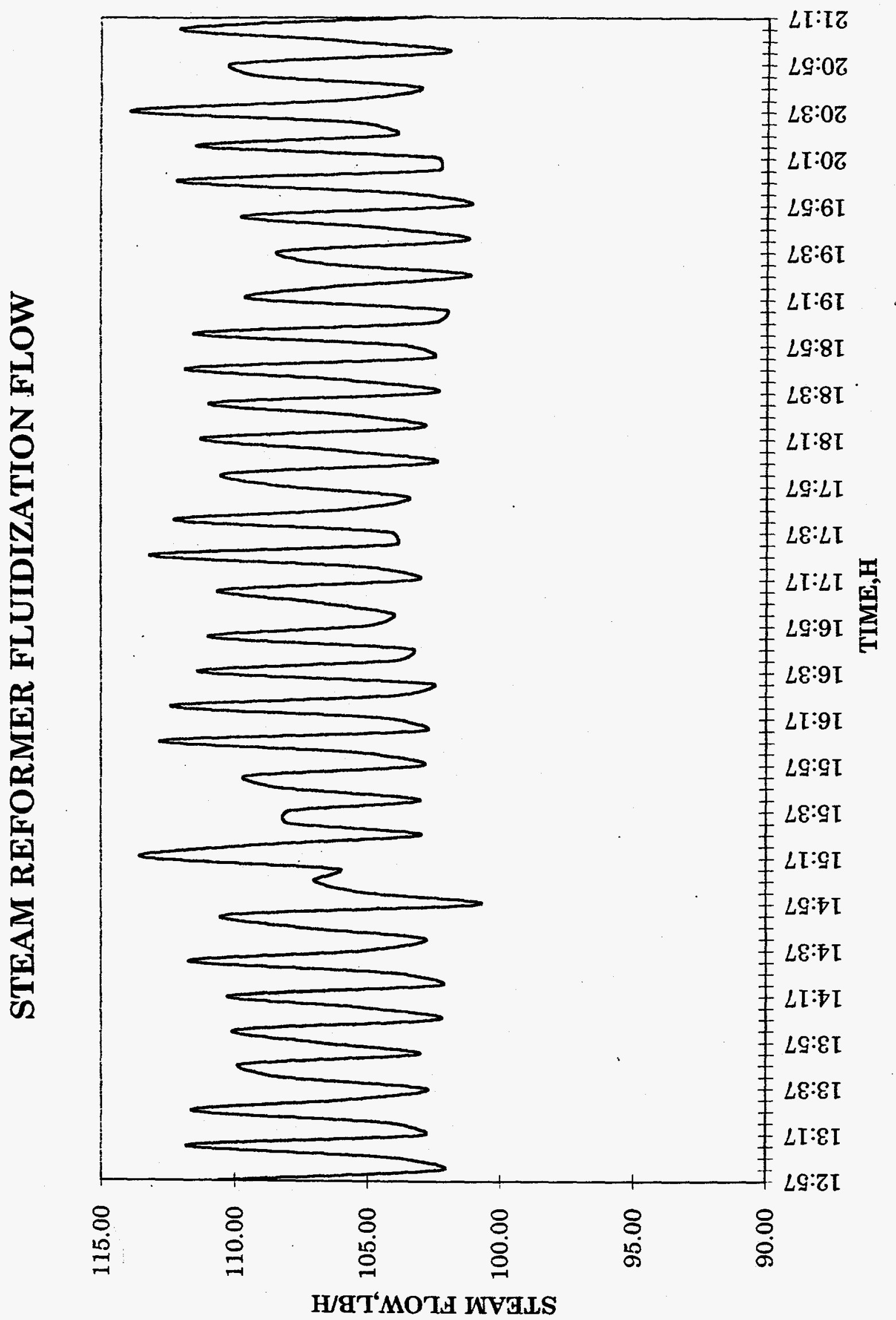




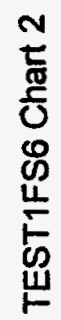

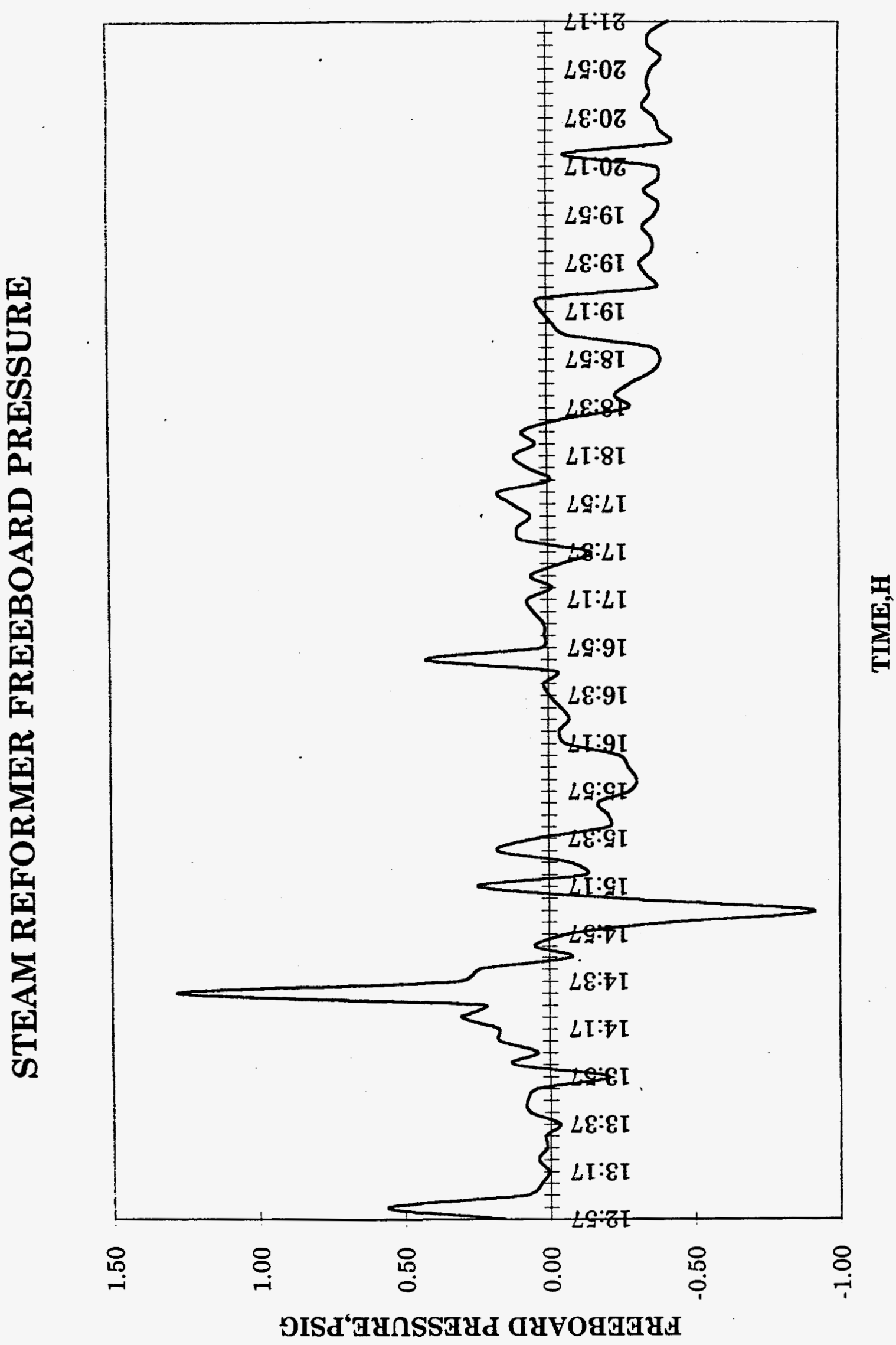




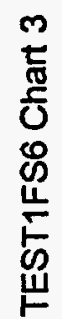
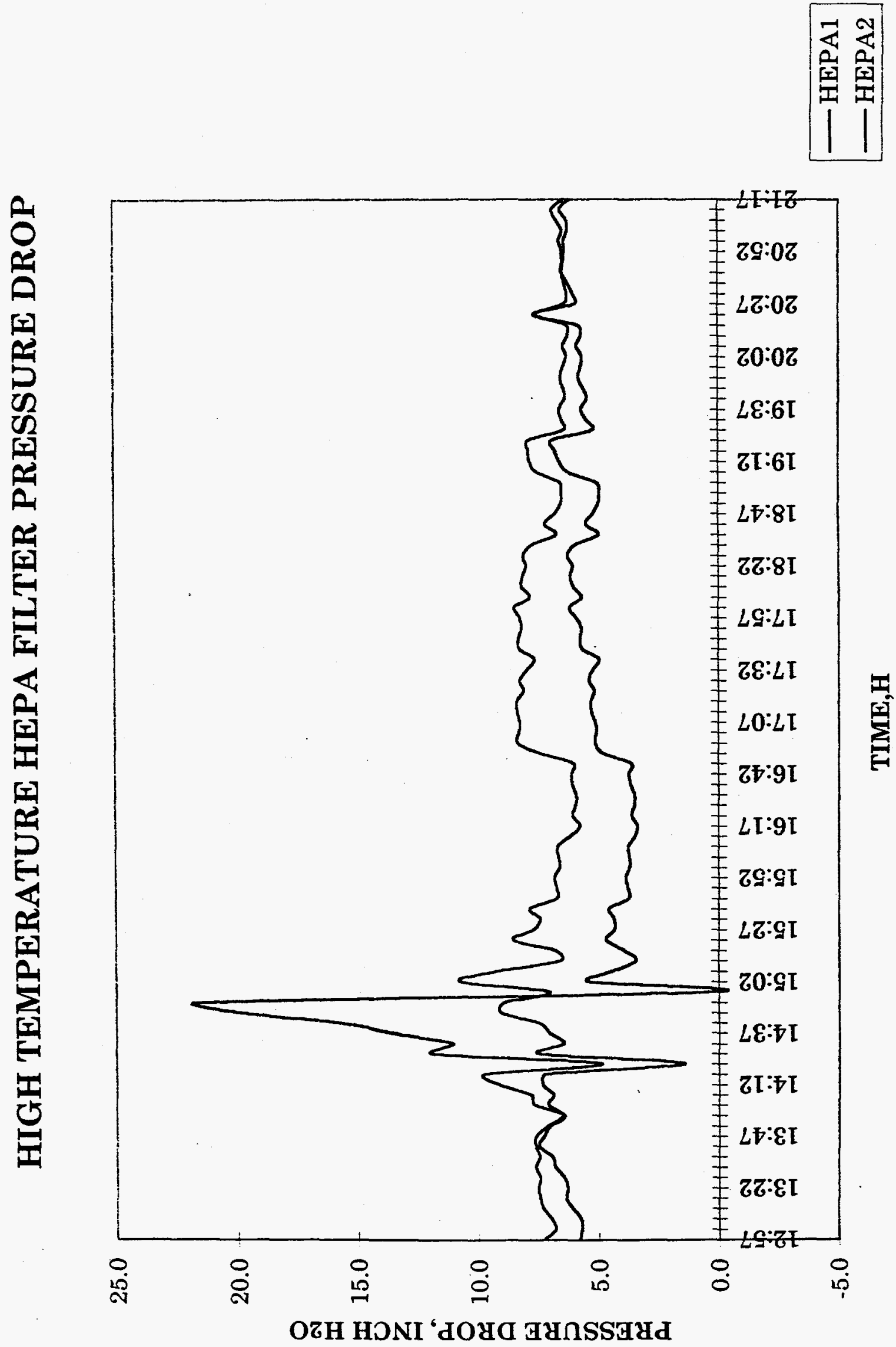


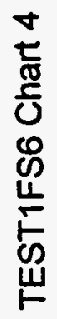
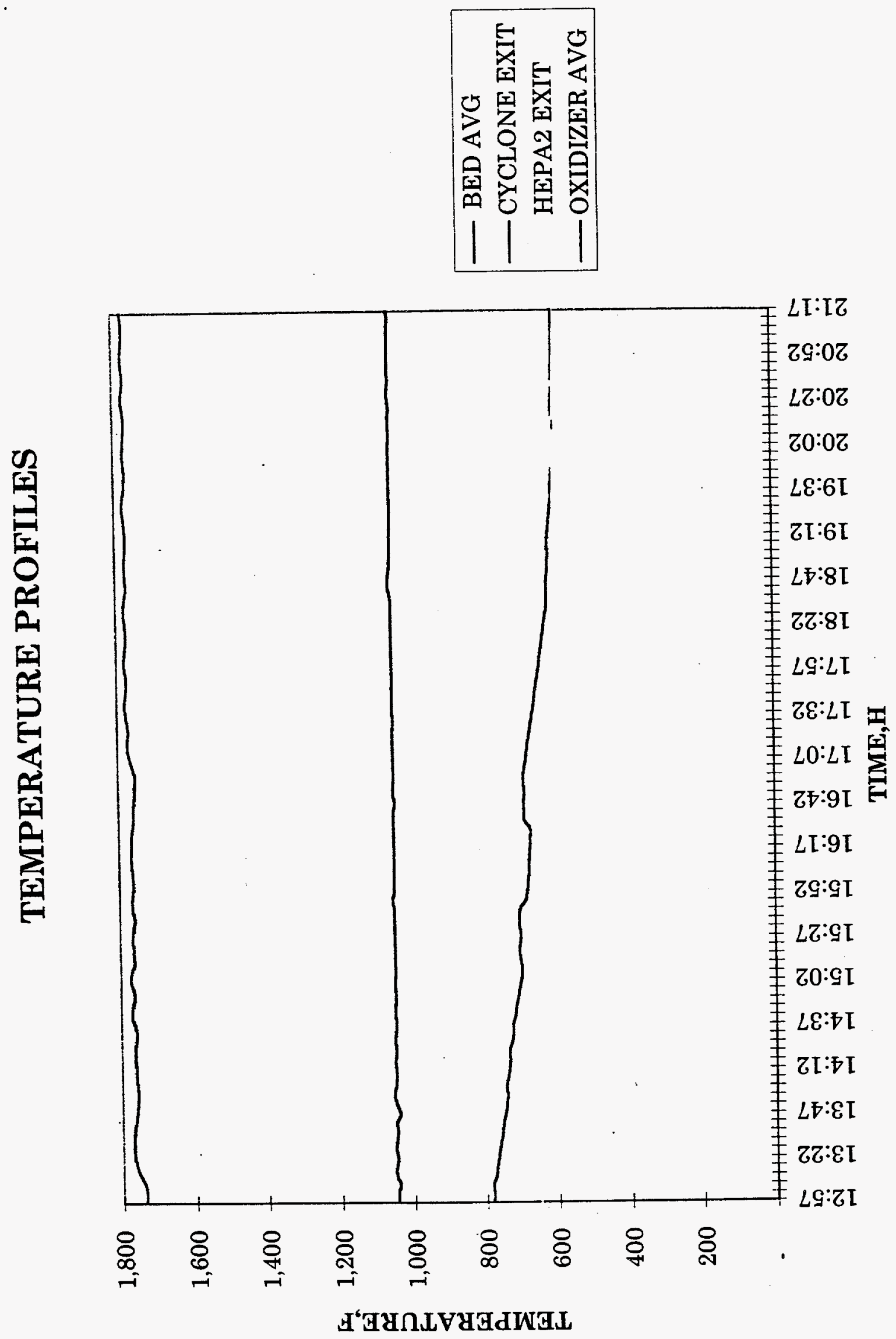


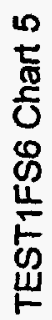

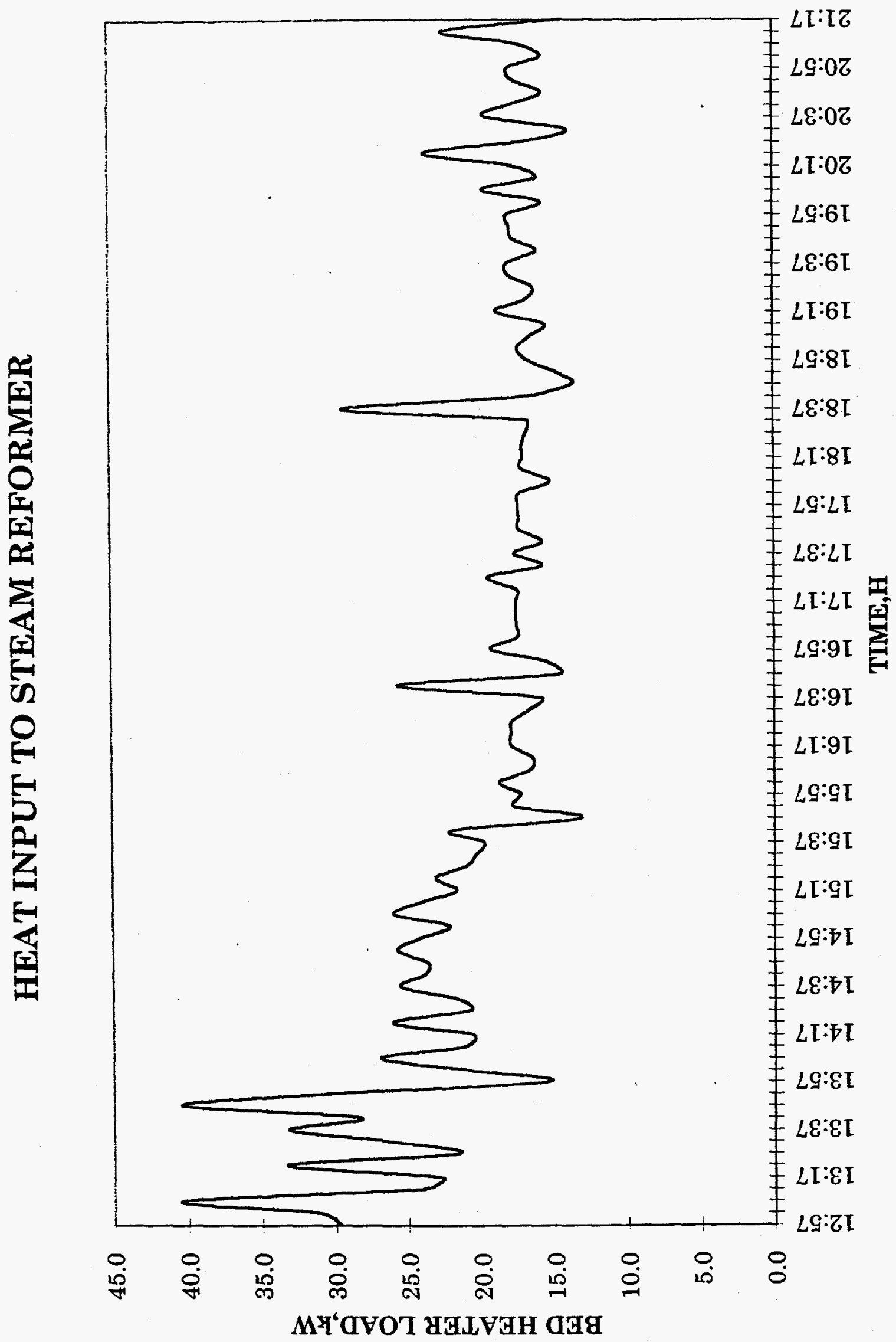




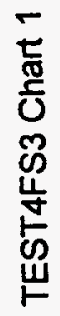

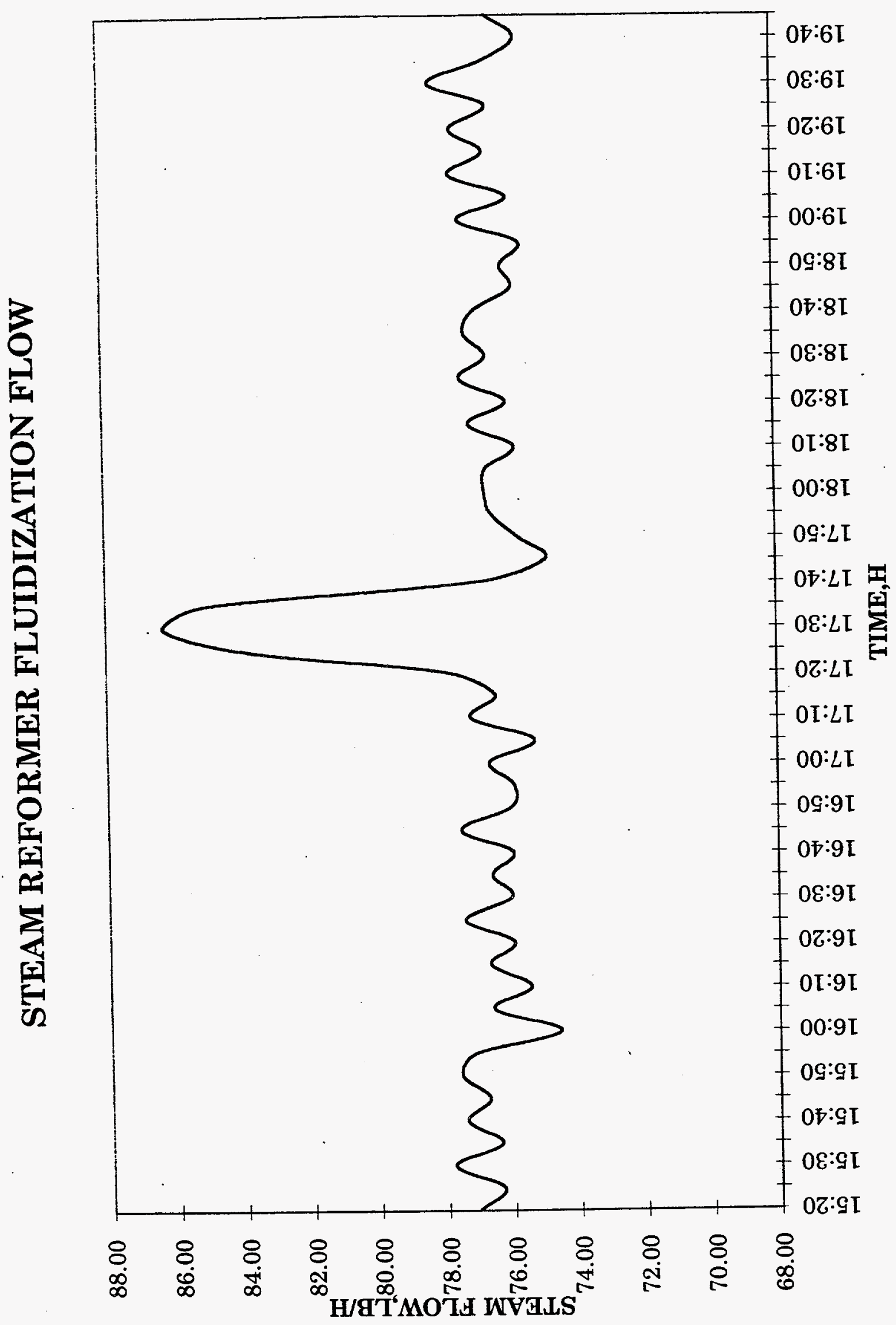




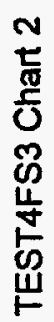

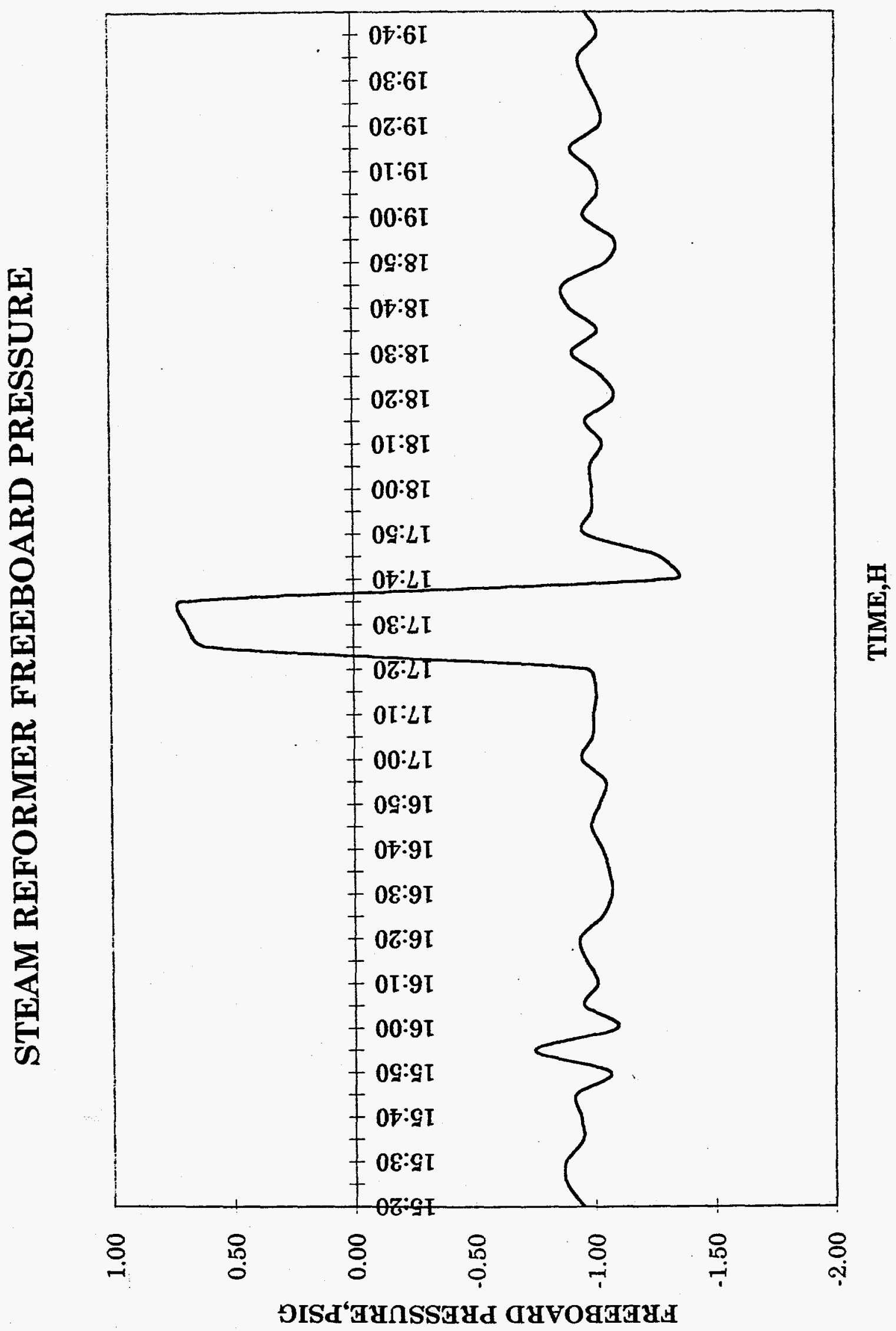




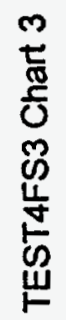
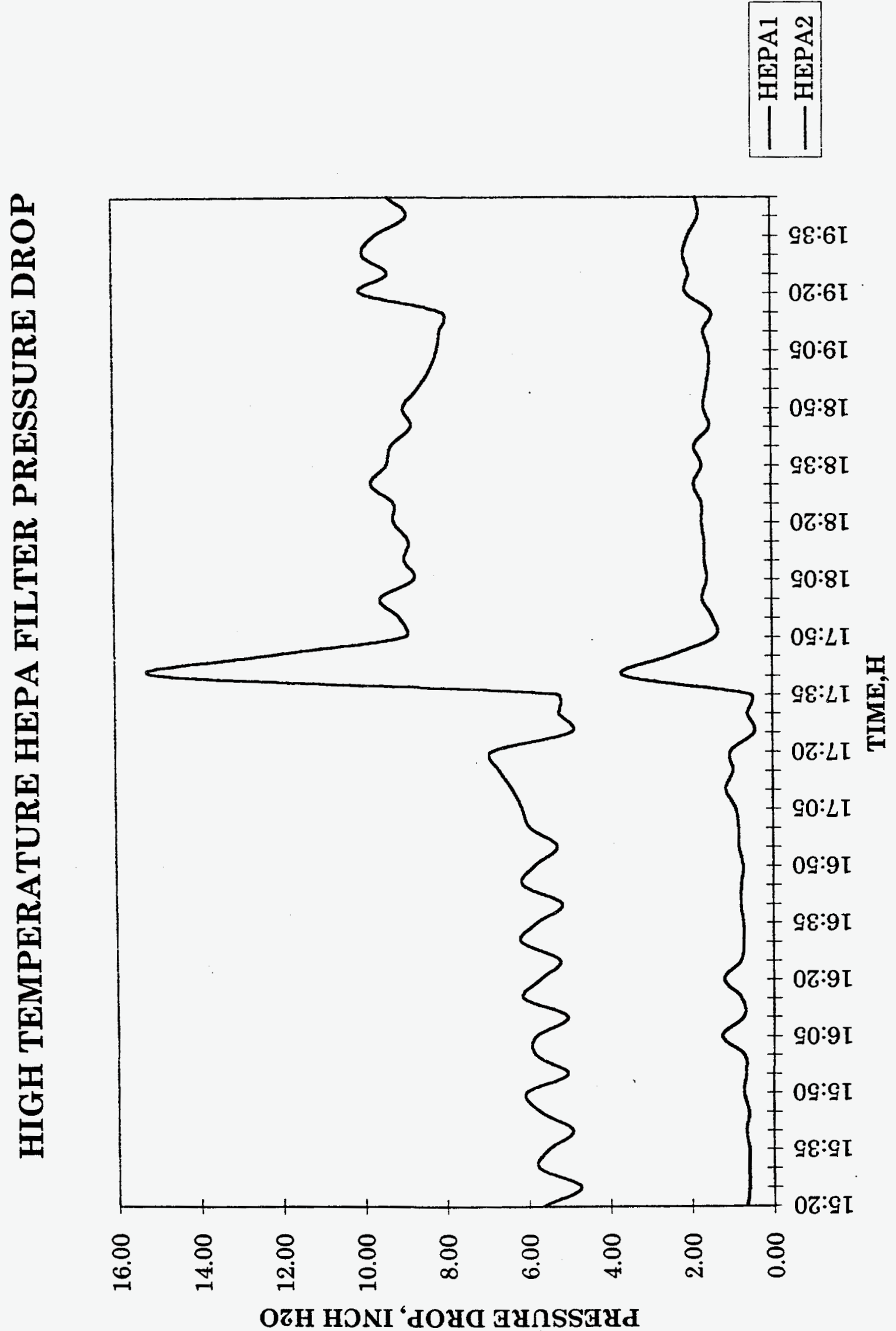

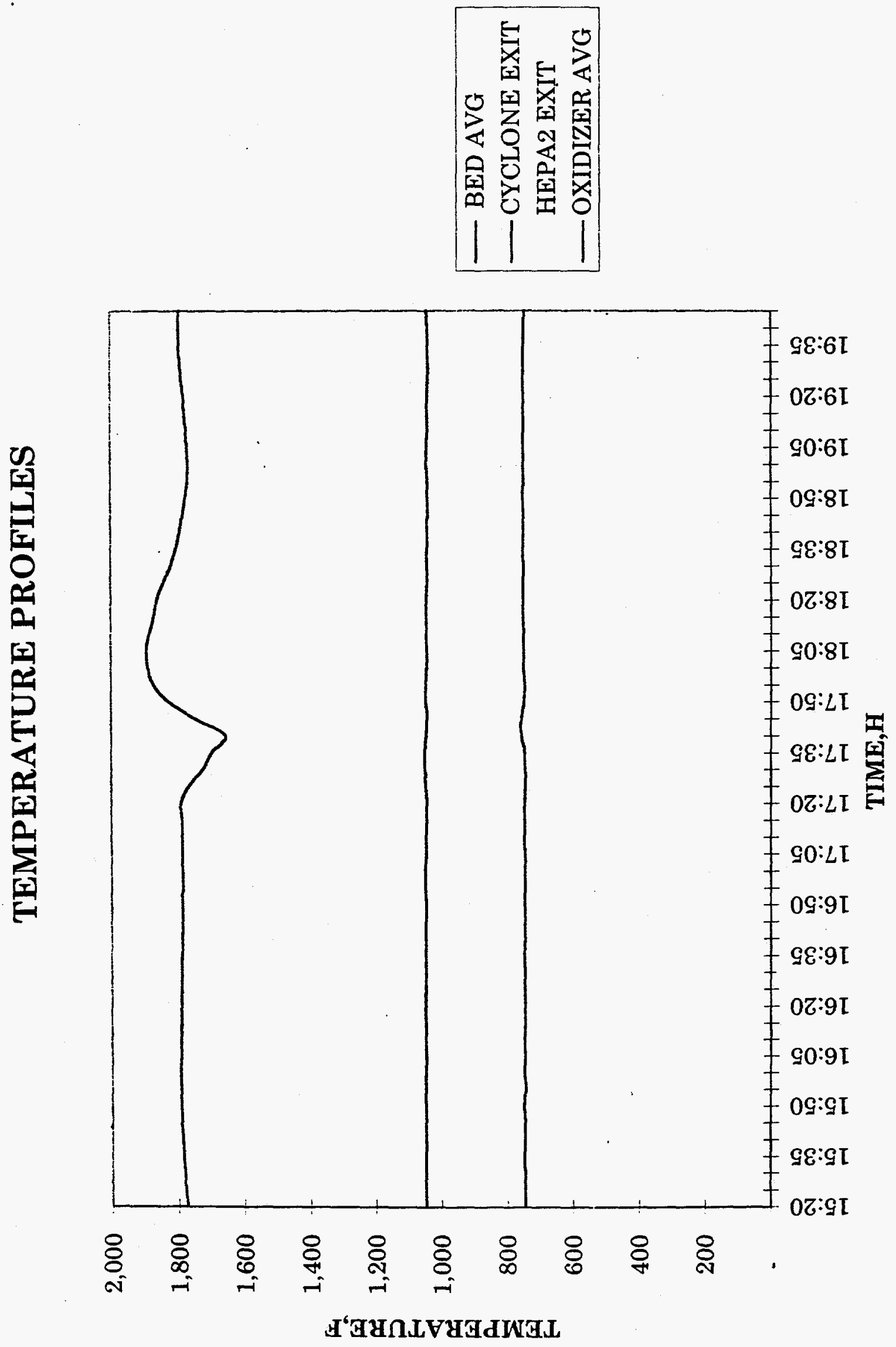


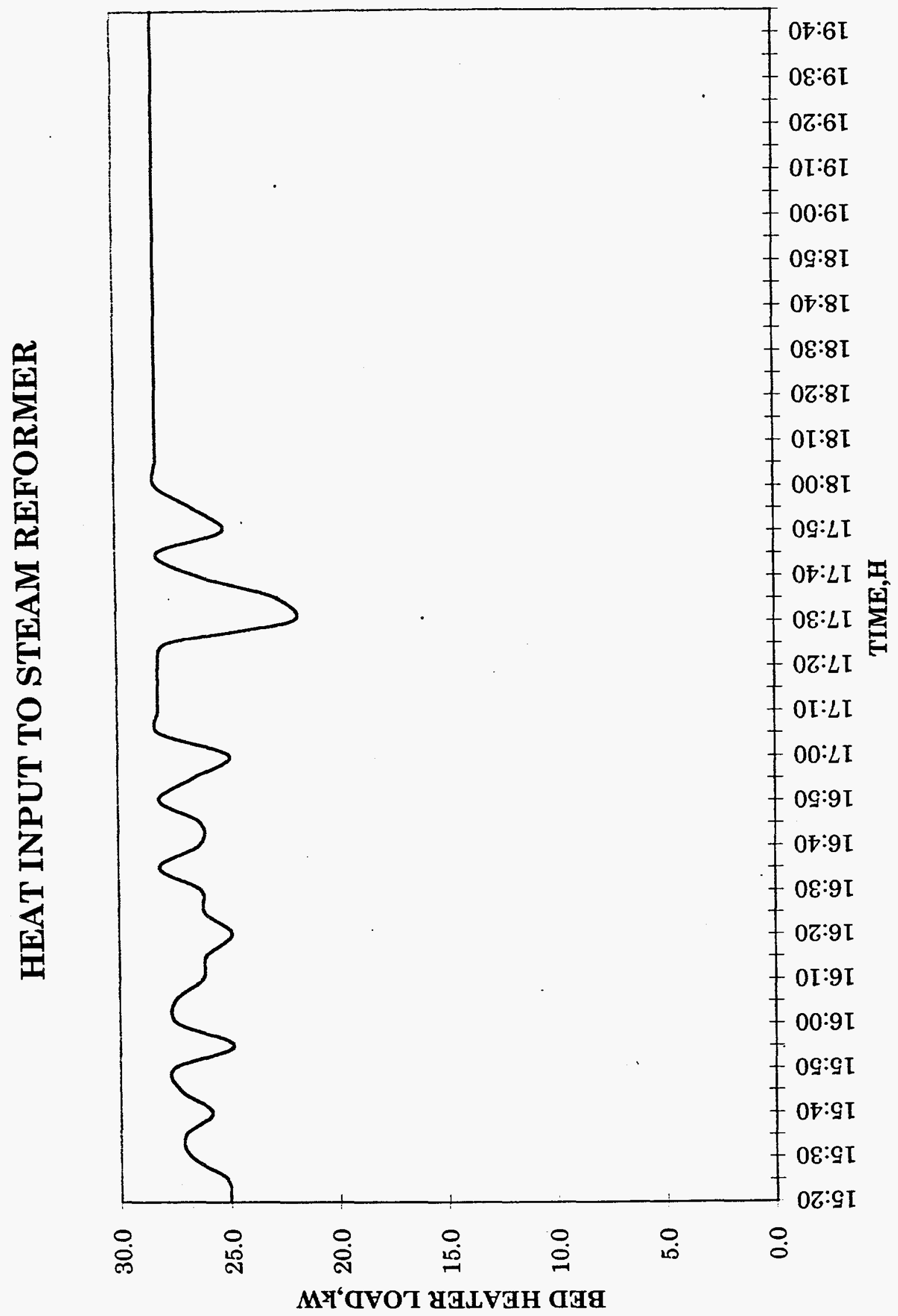




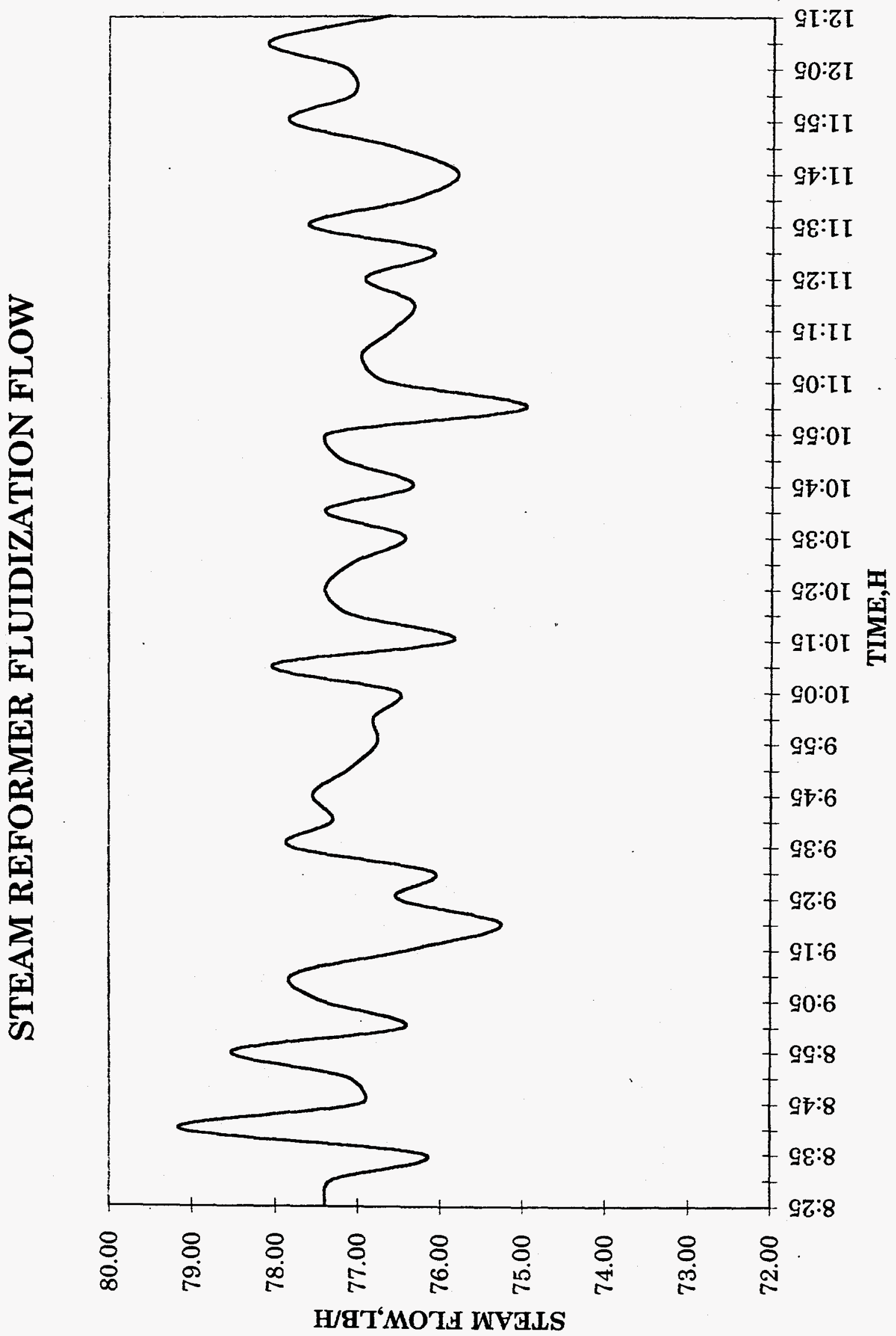




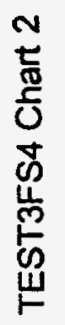

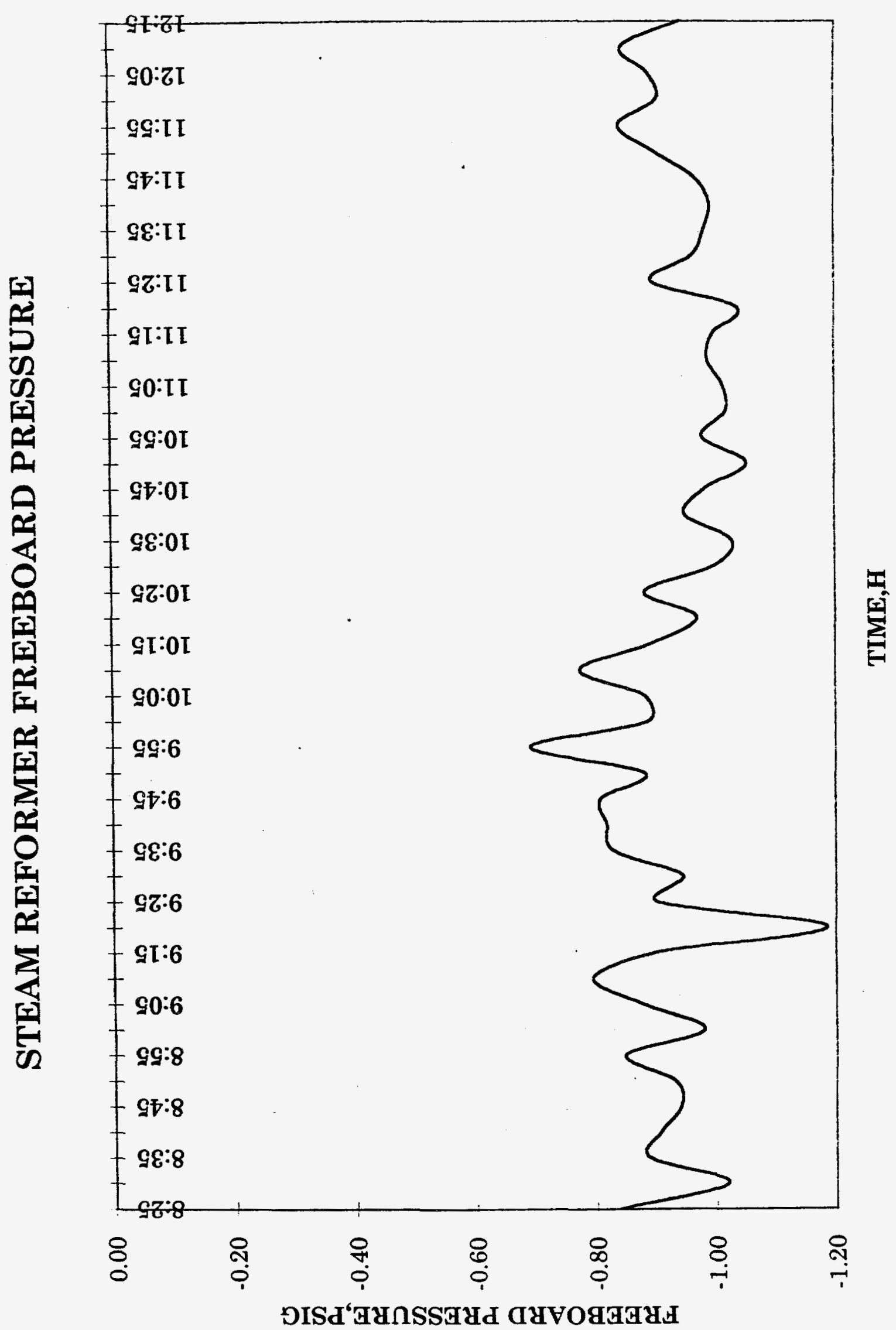




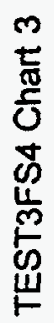
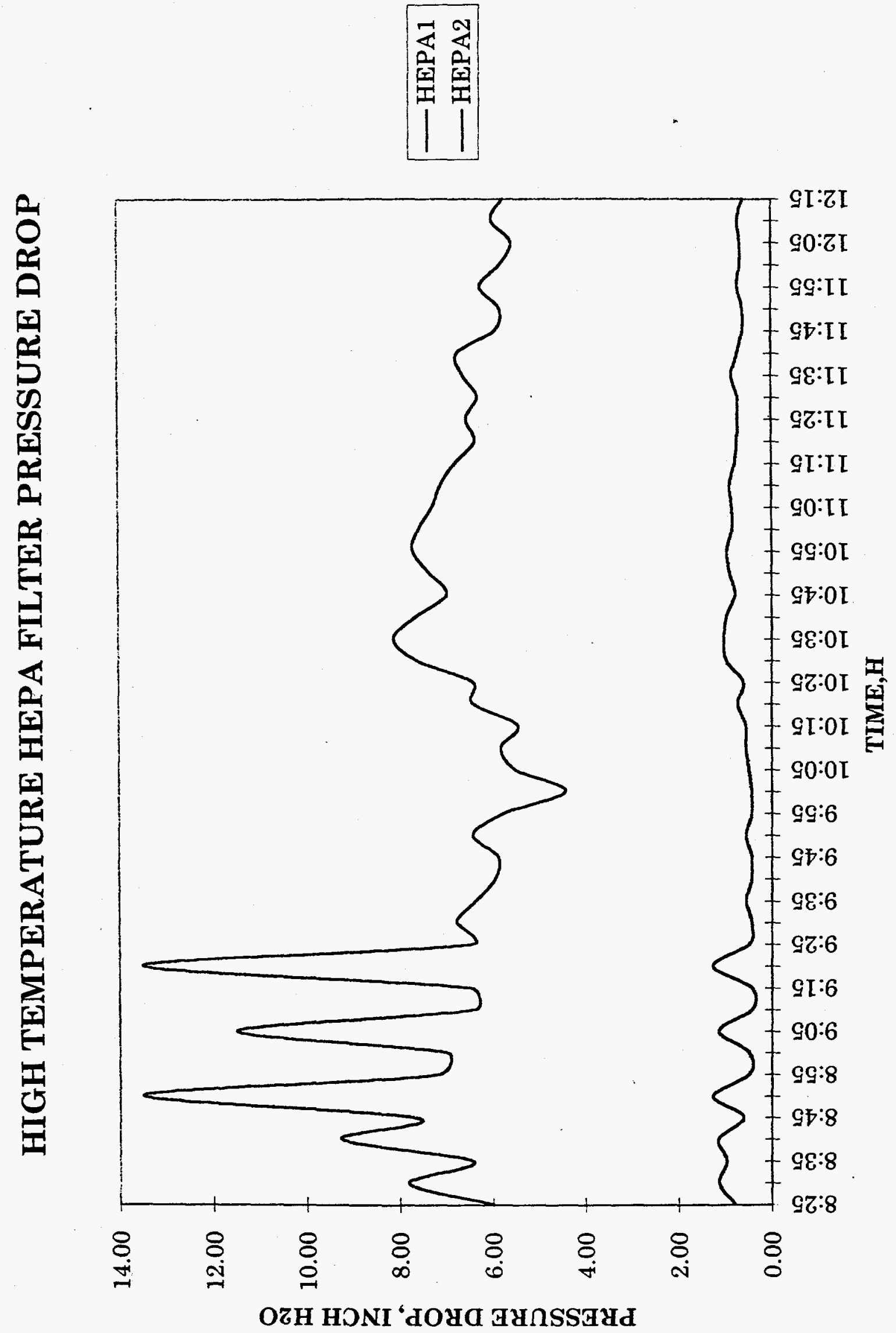


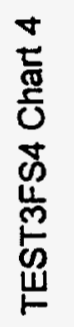
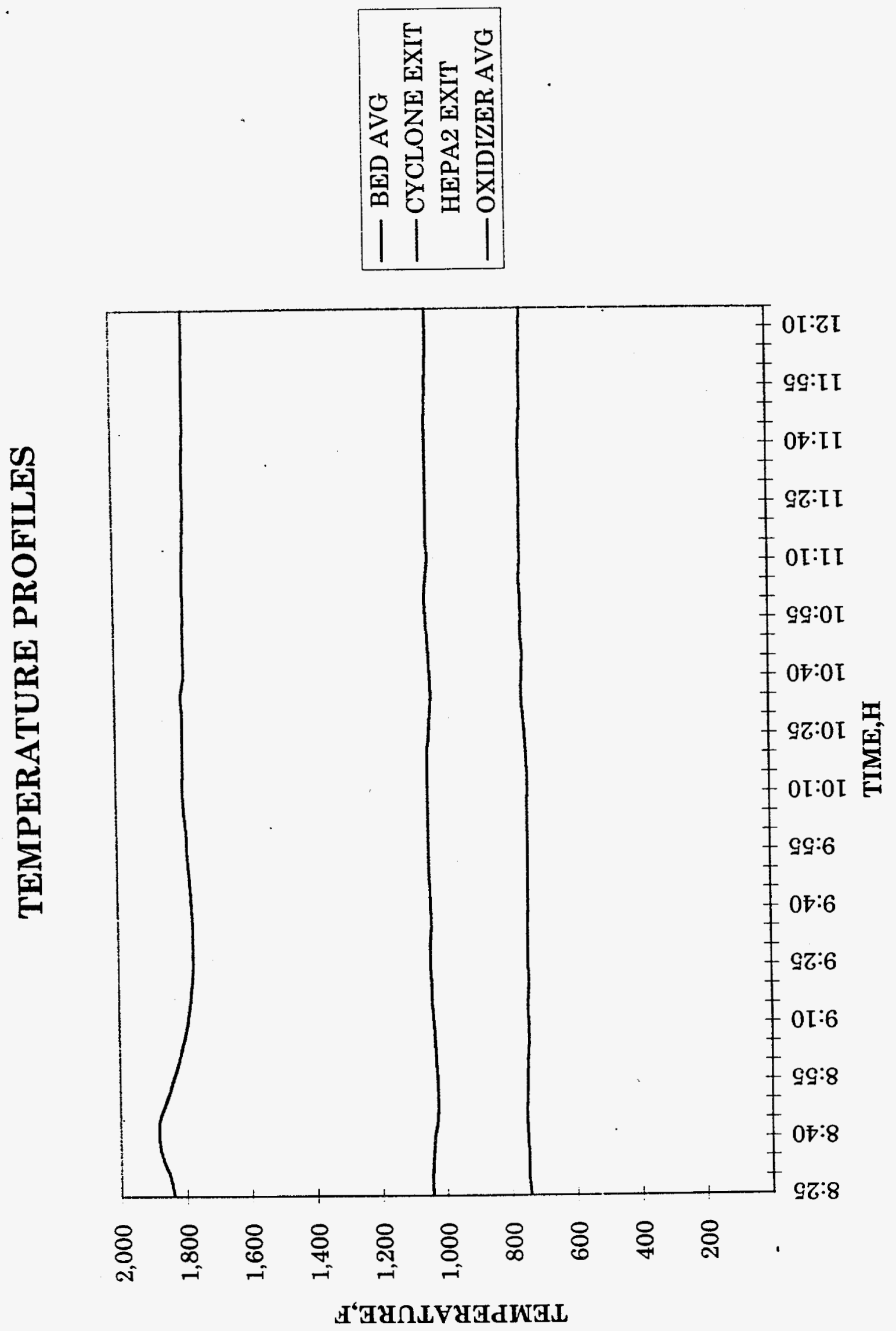


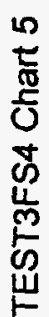

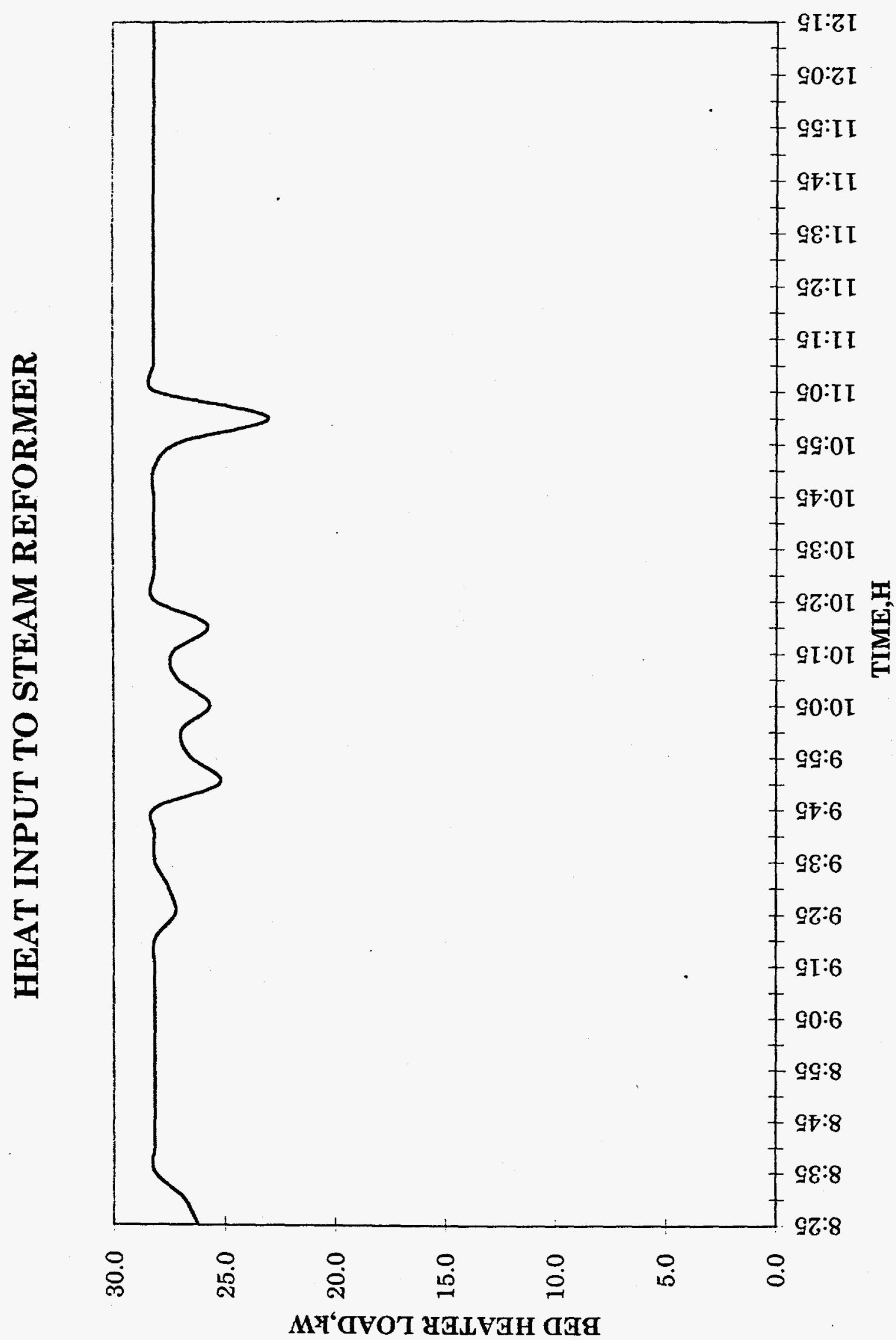




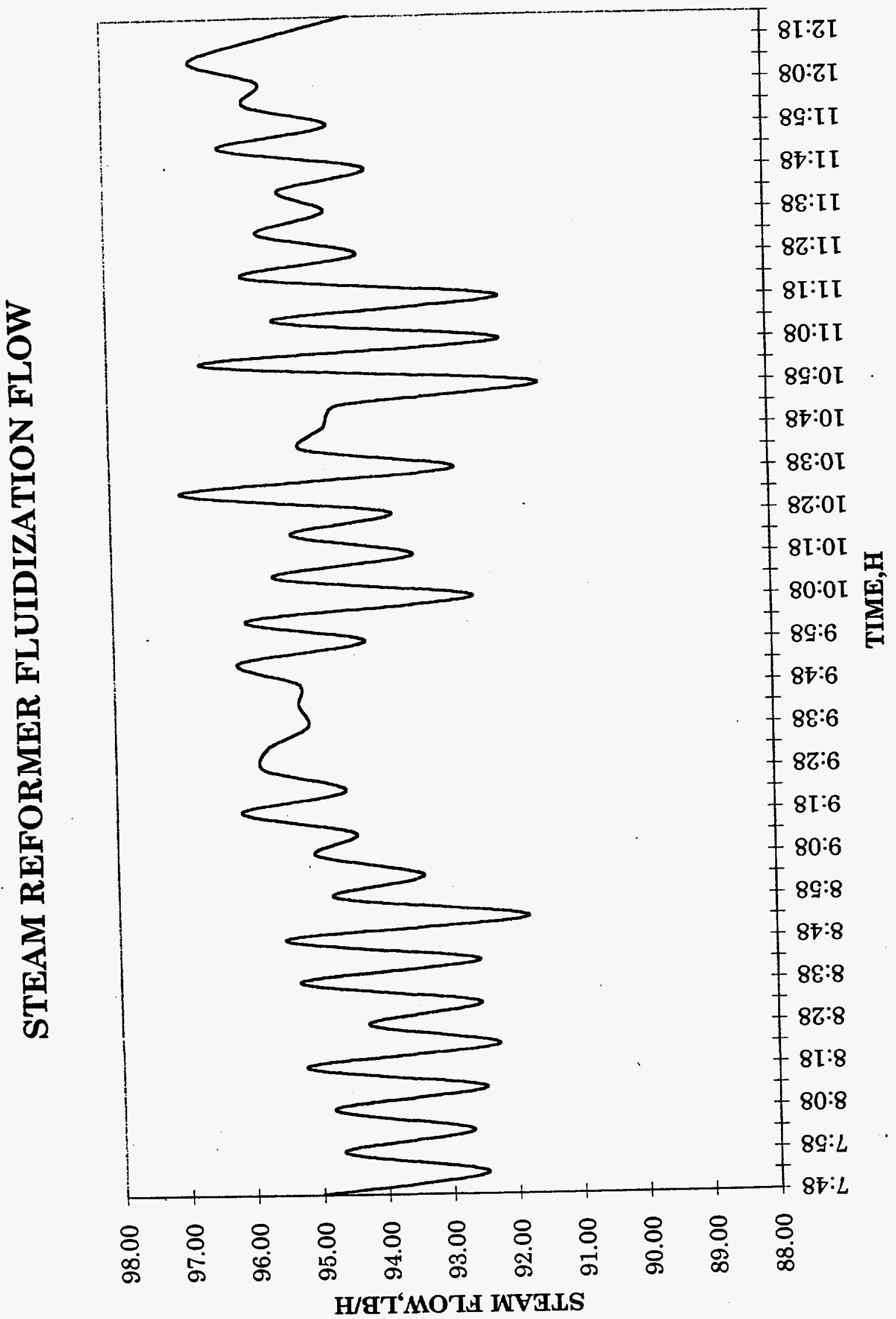




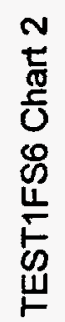

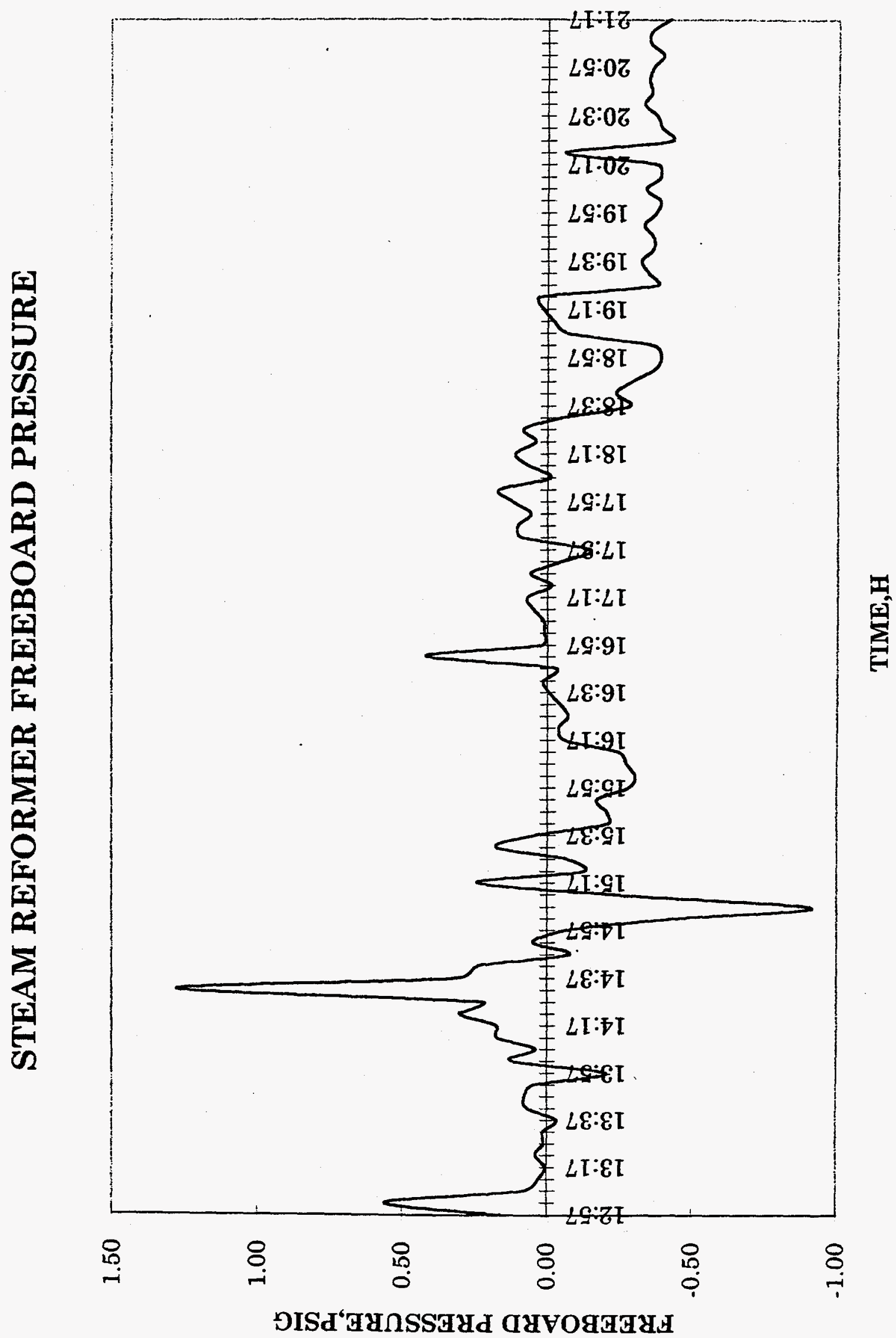




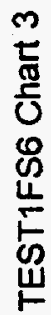
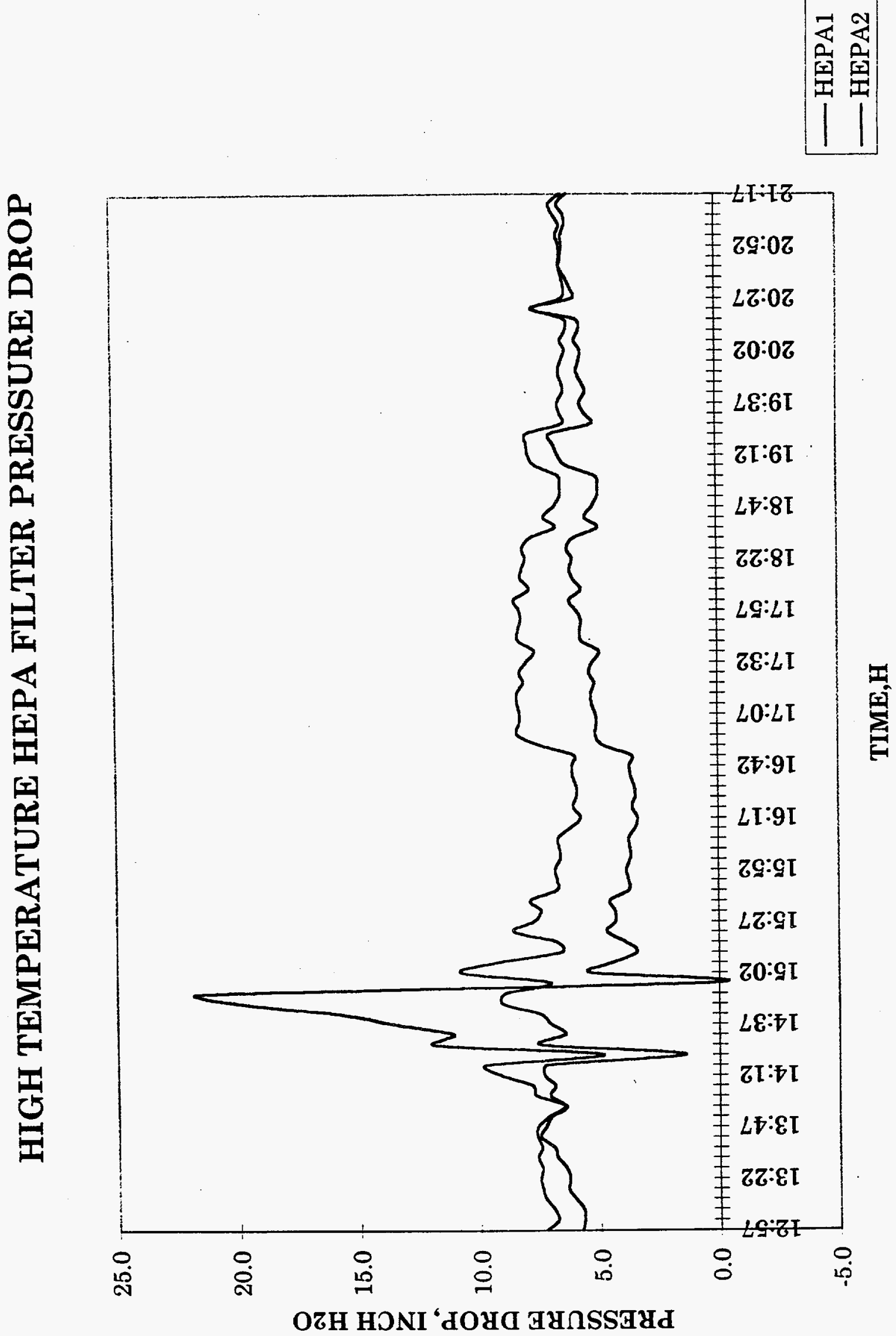

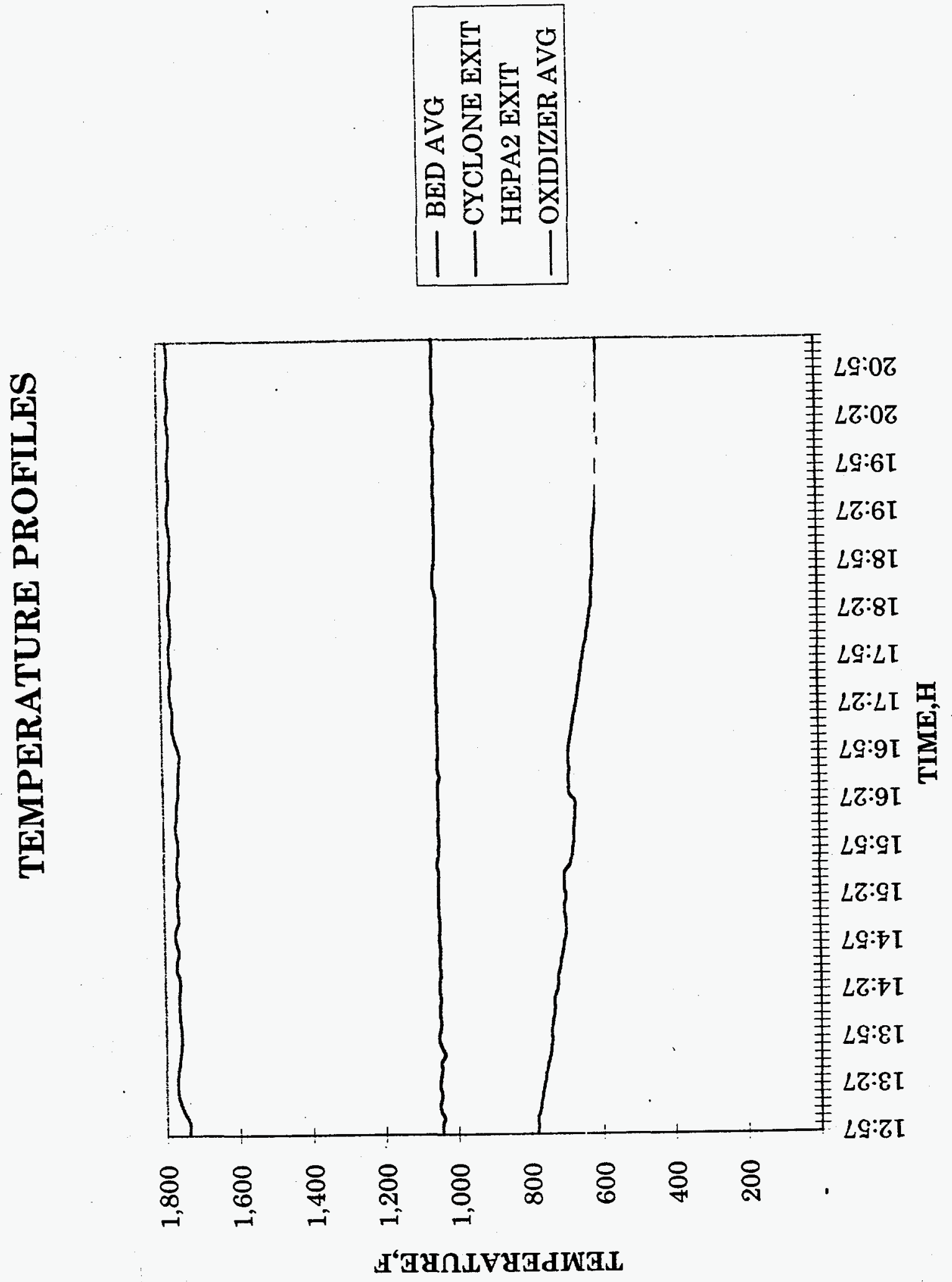


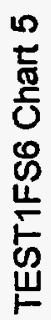

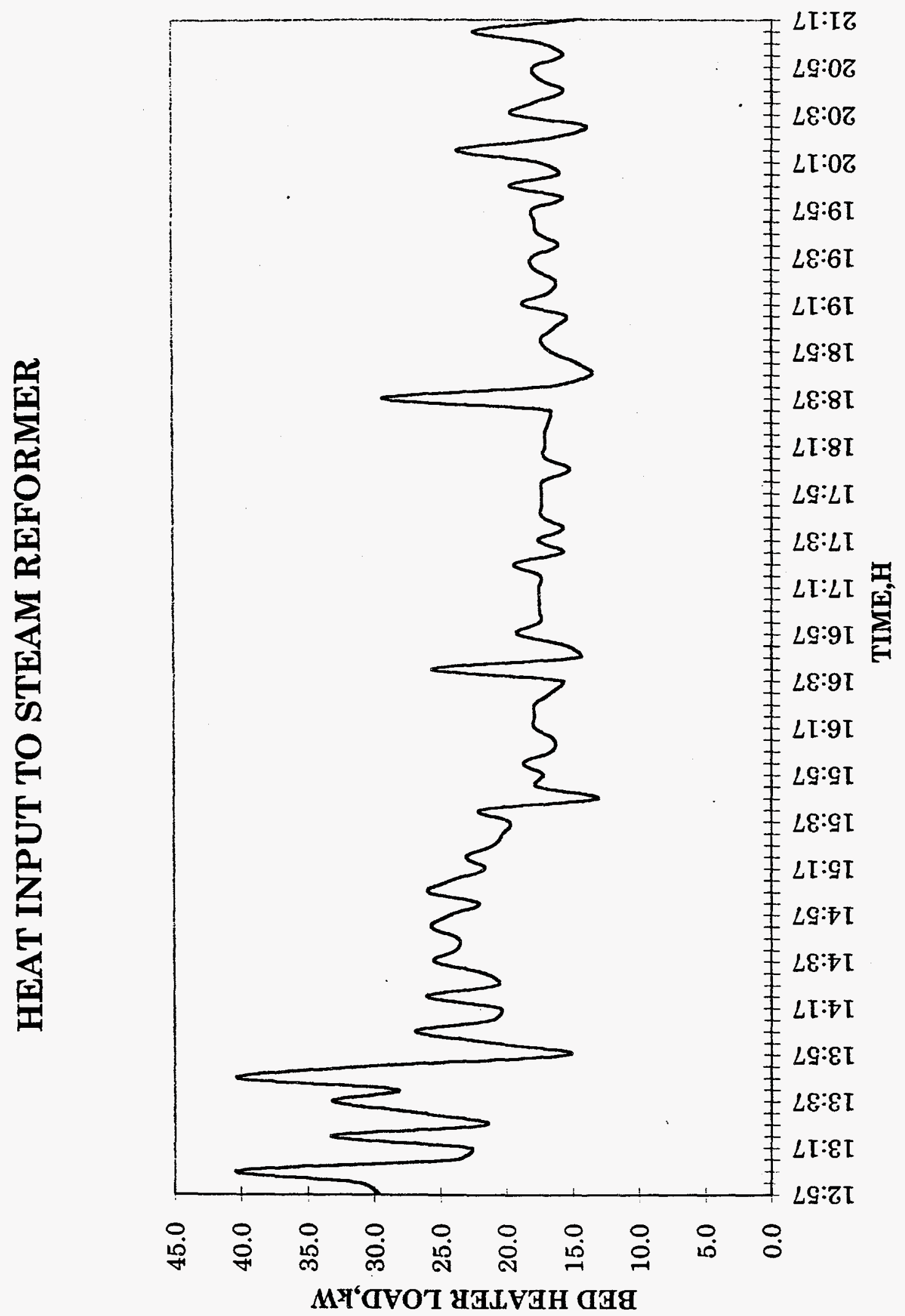


PIKETON WASTE SIMULATION TESTS 


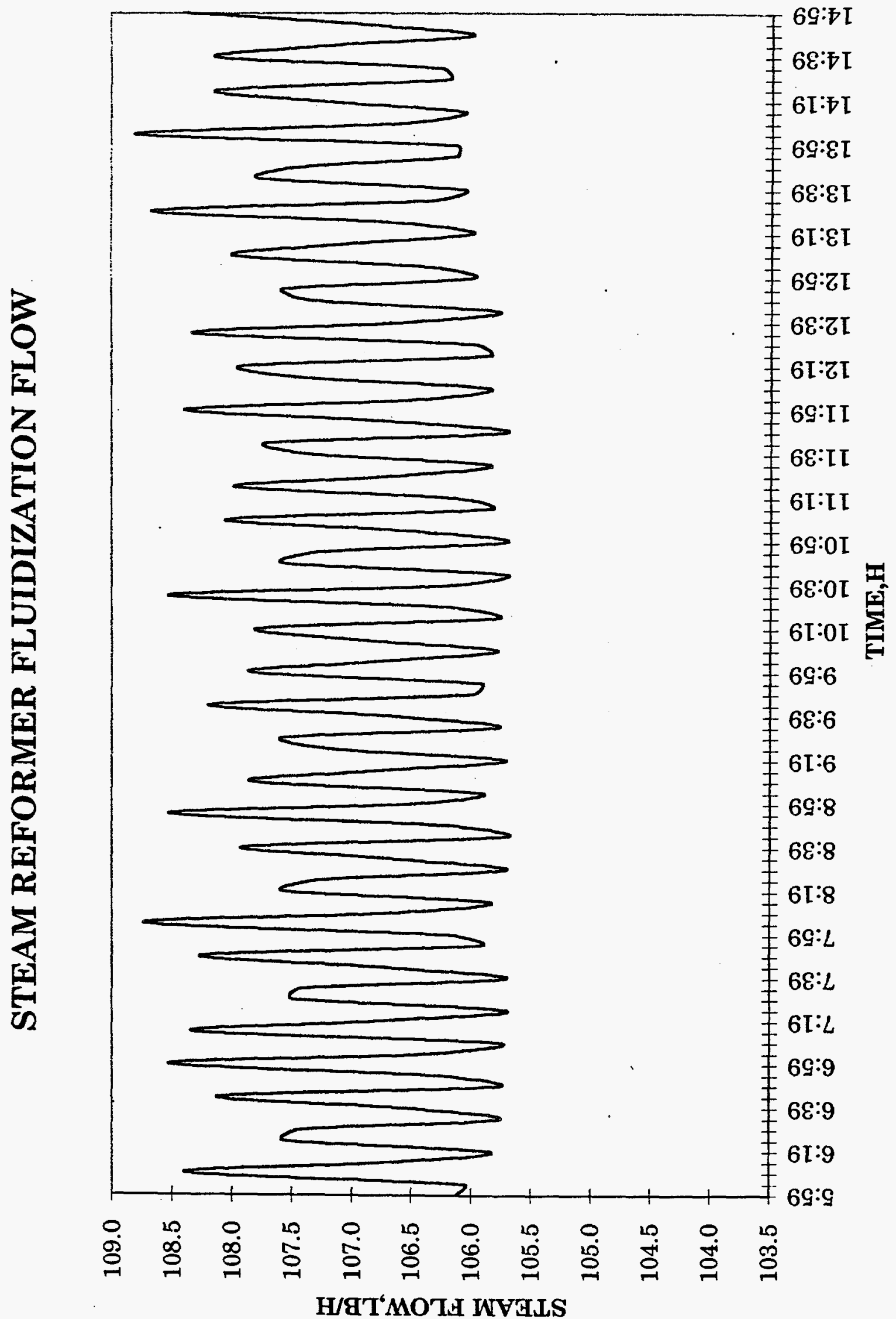




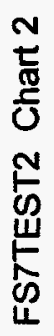

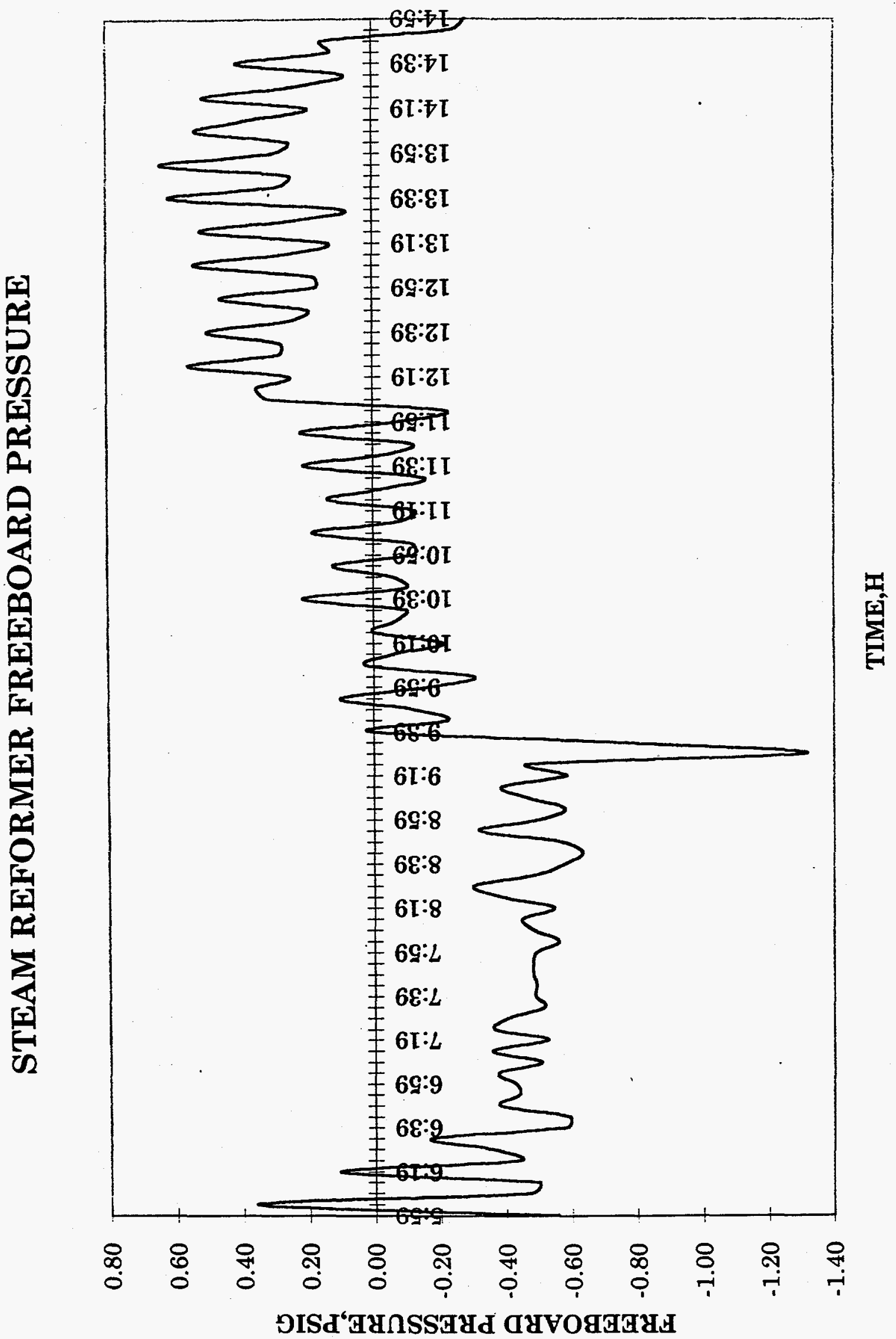




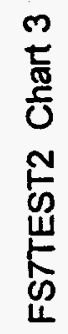
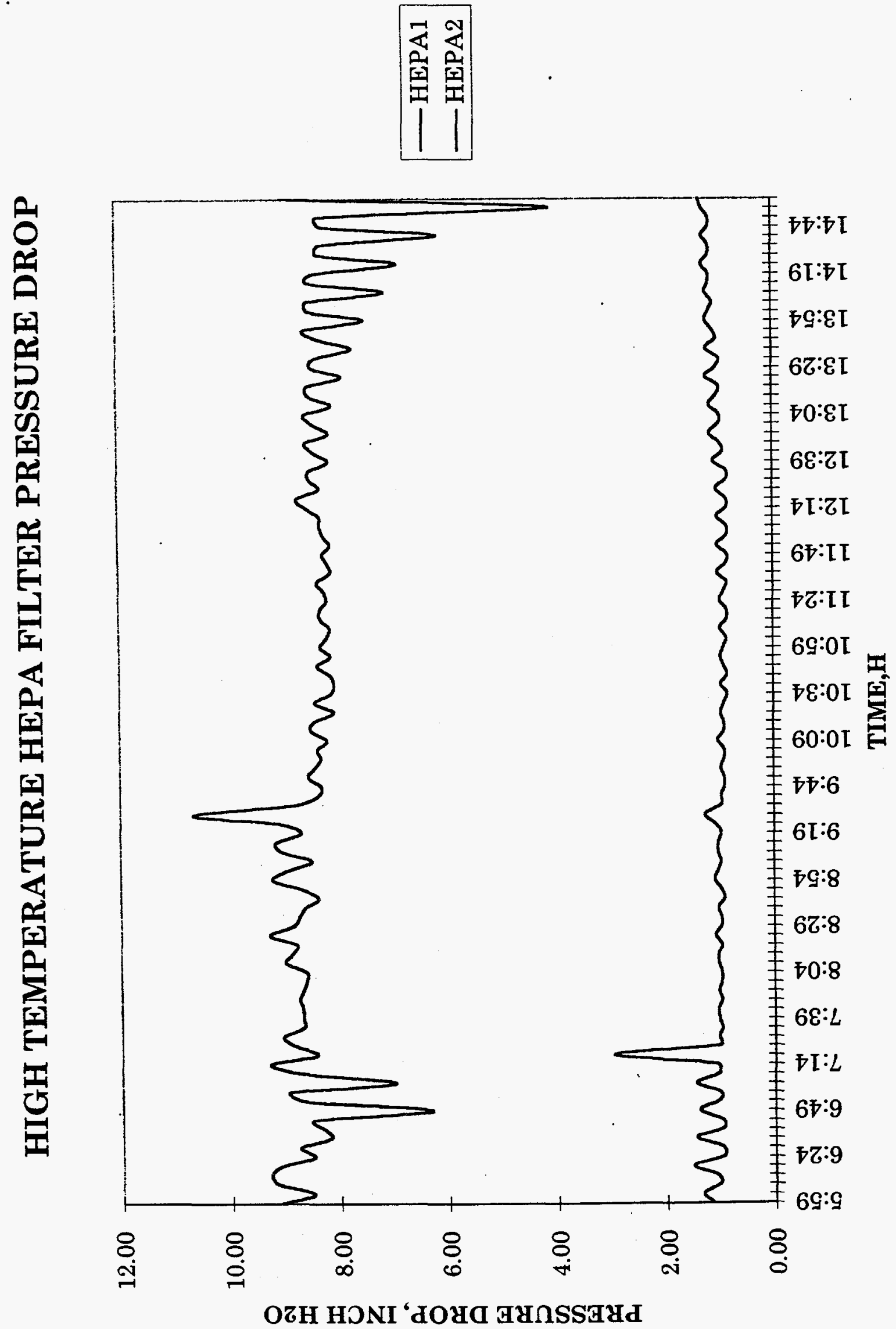


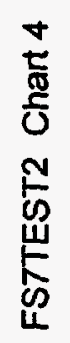

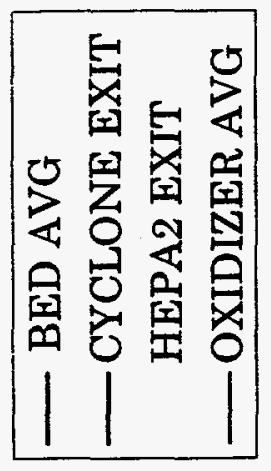

[1]

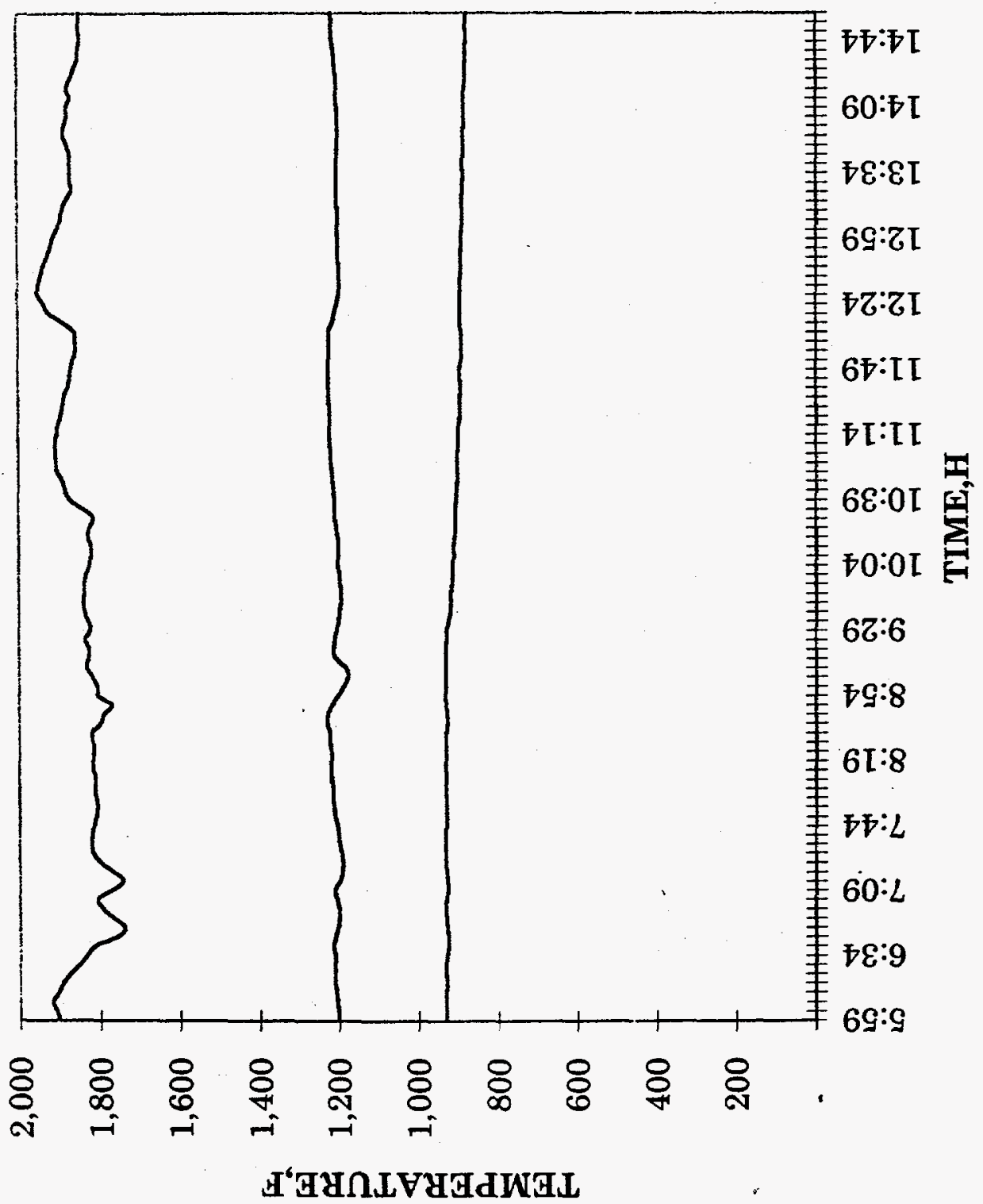




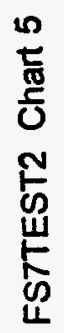

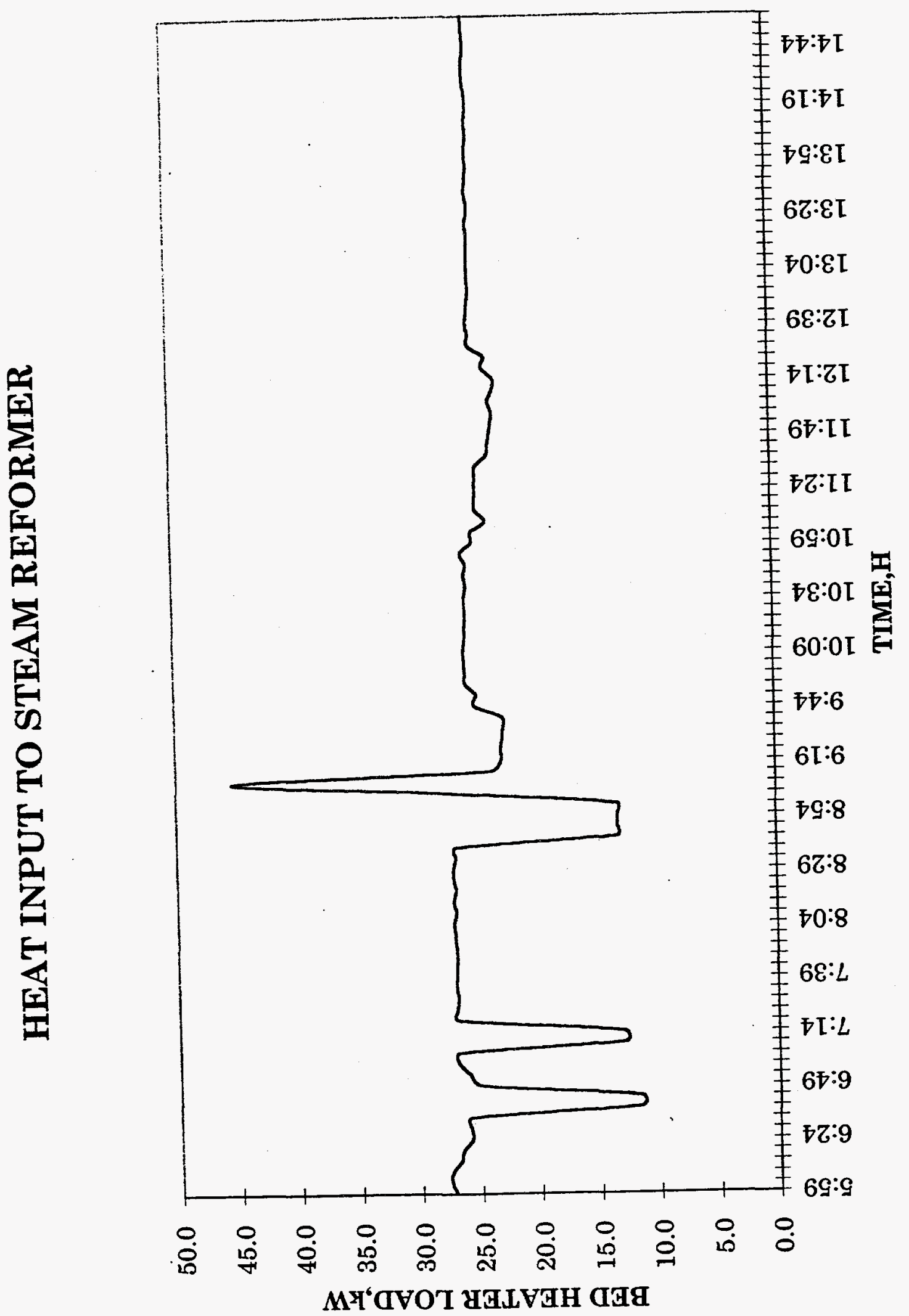




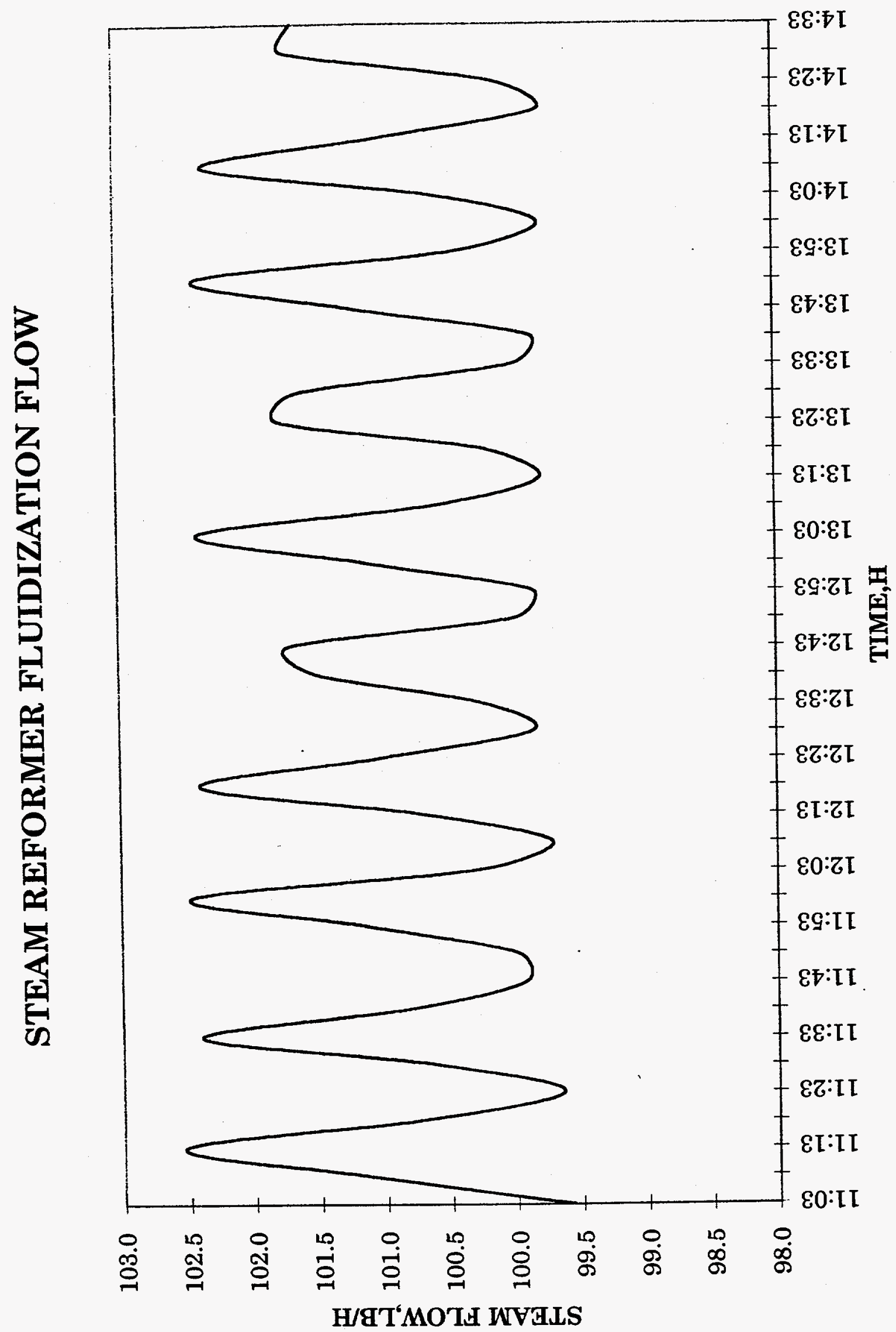




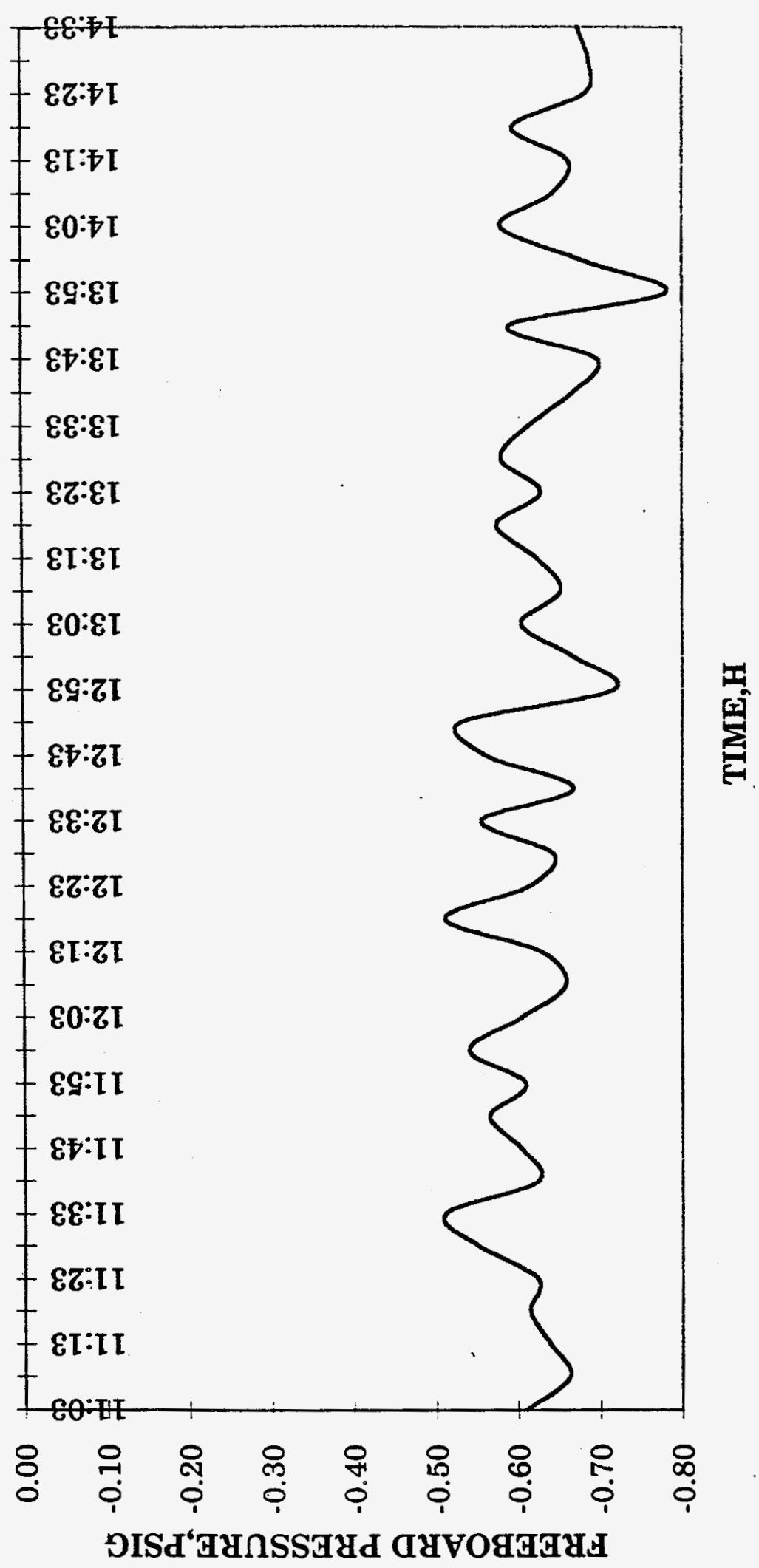


$m$
$\frac{1}{D}$
$\frac{5}{0}$
$m$
$\frac{5}{W}$
$\frac{\pi}{4}$
$\frac{1}{4}$
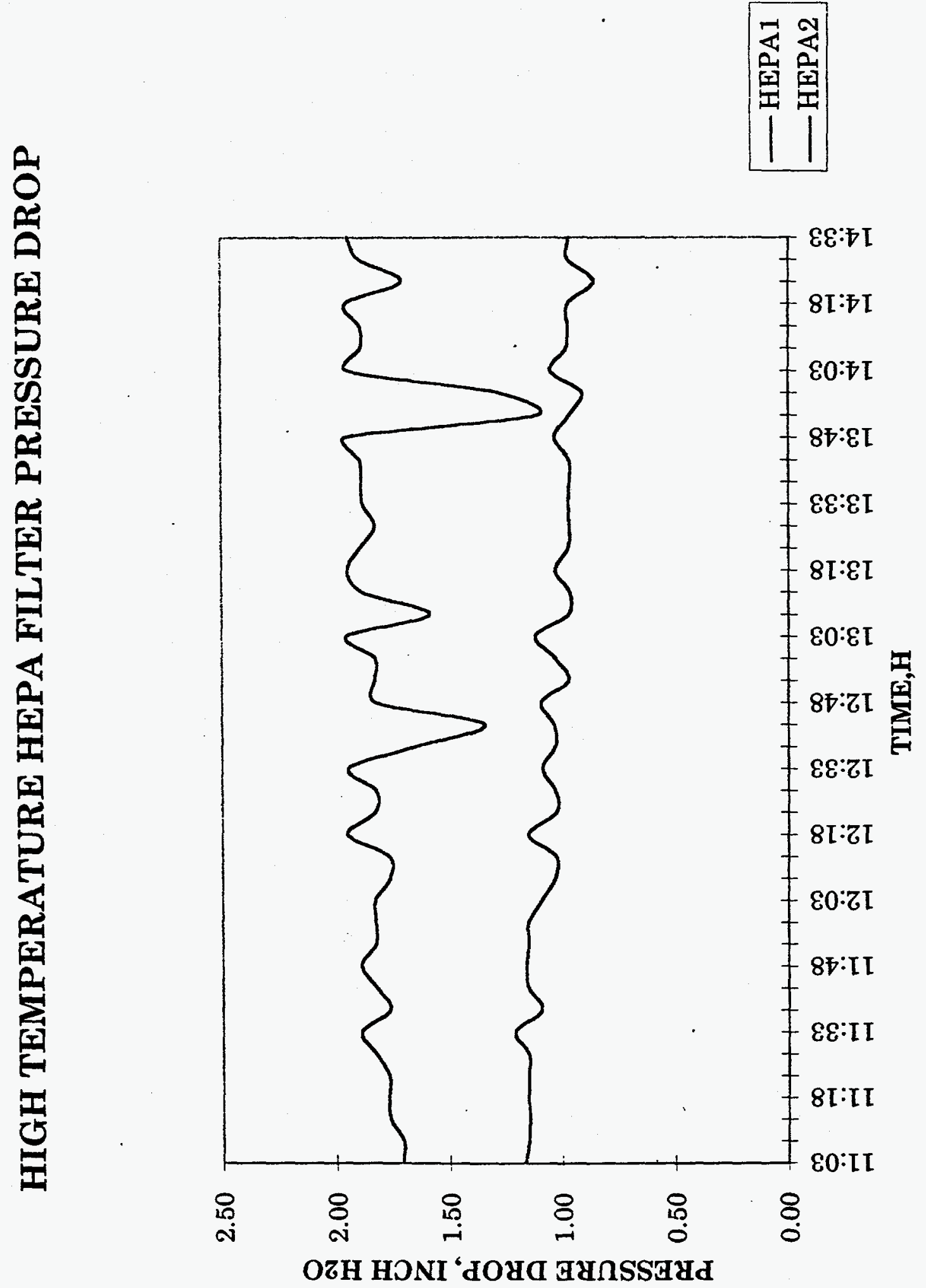


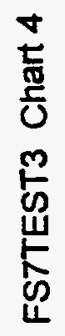
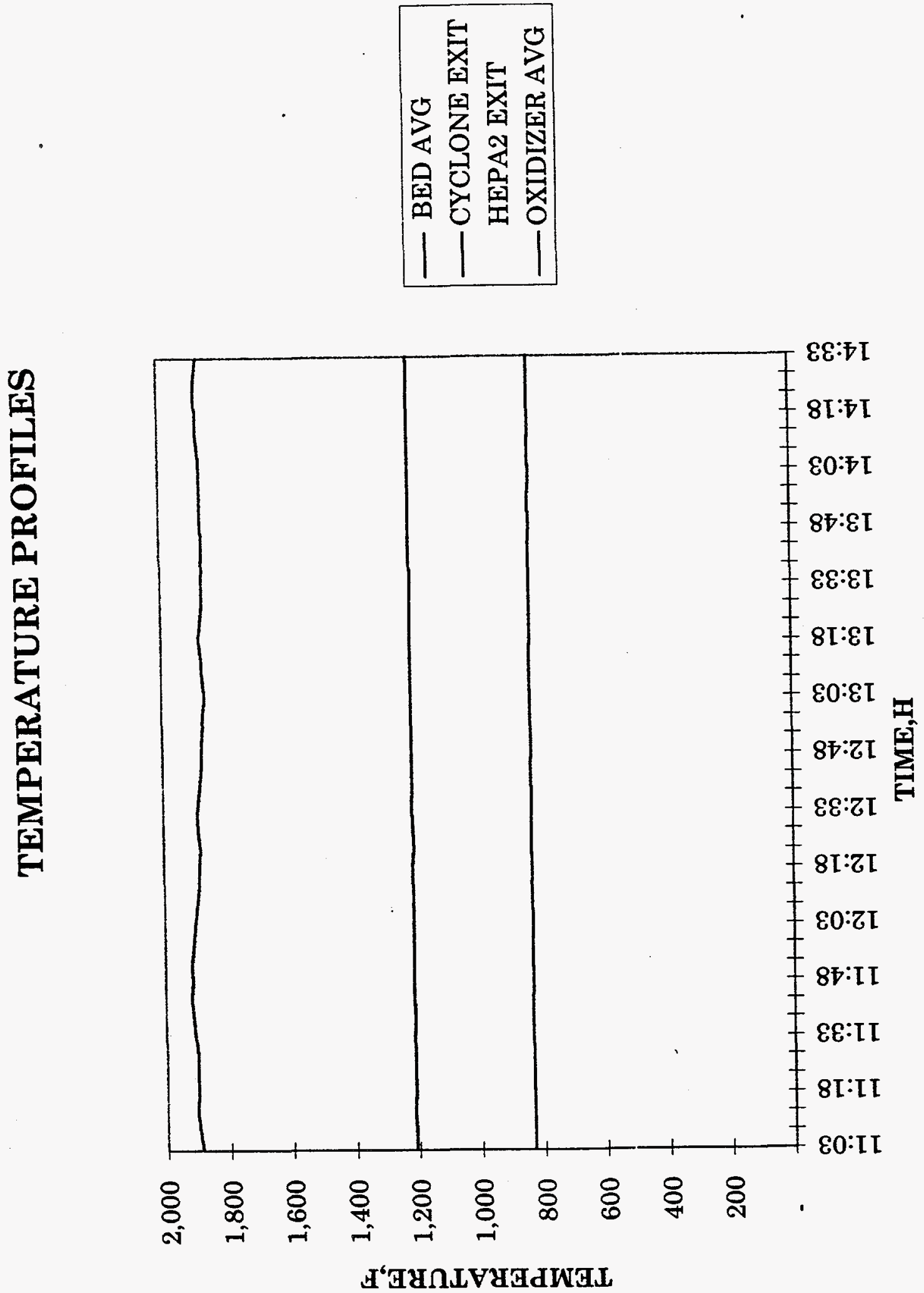


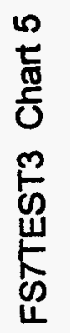

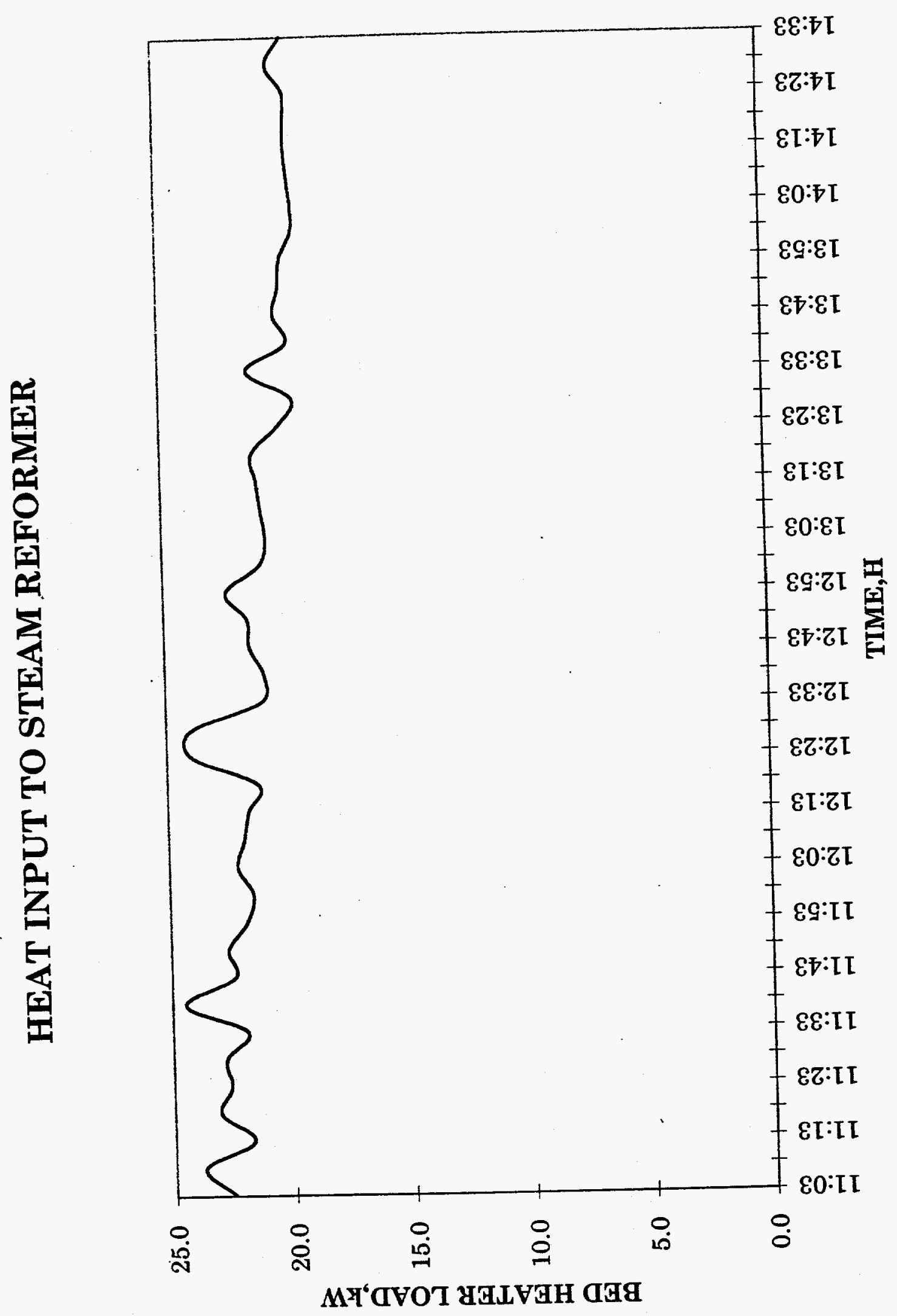




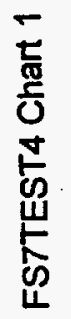

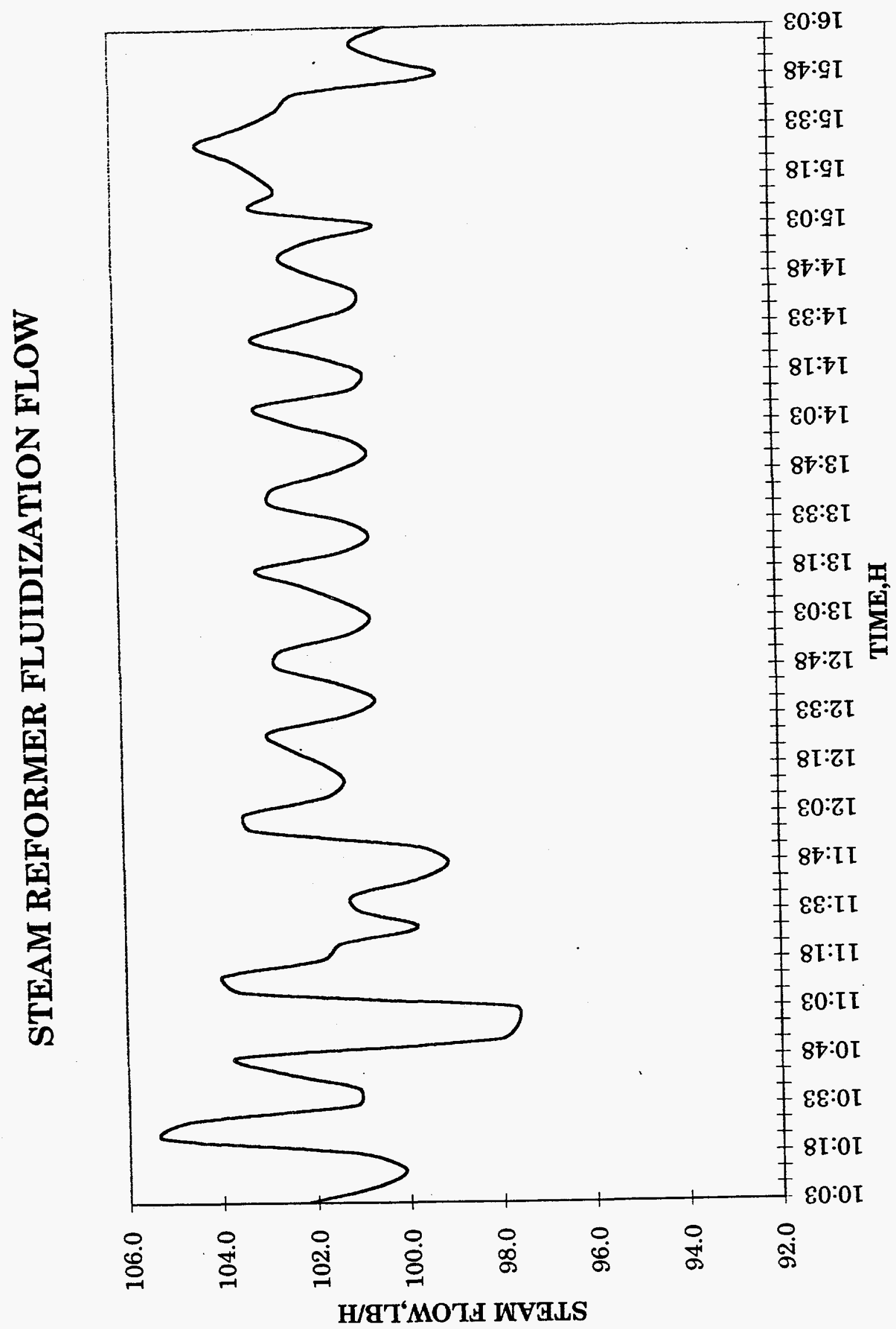




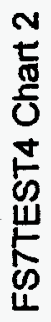

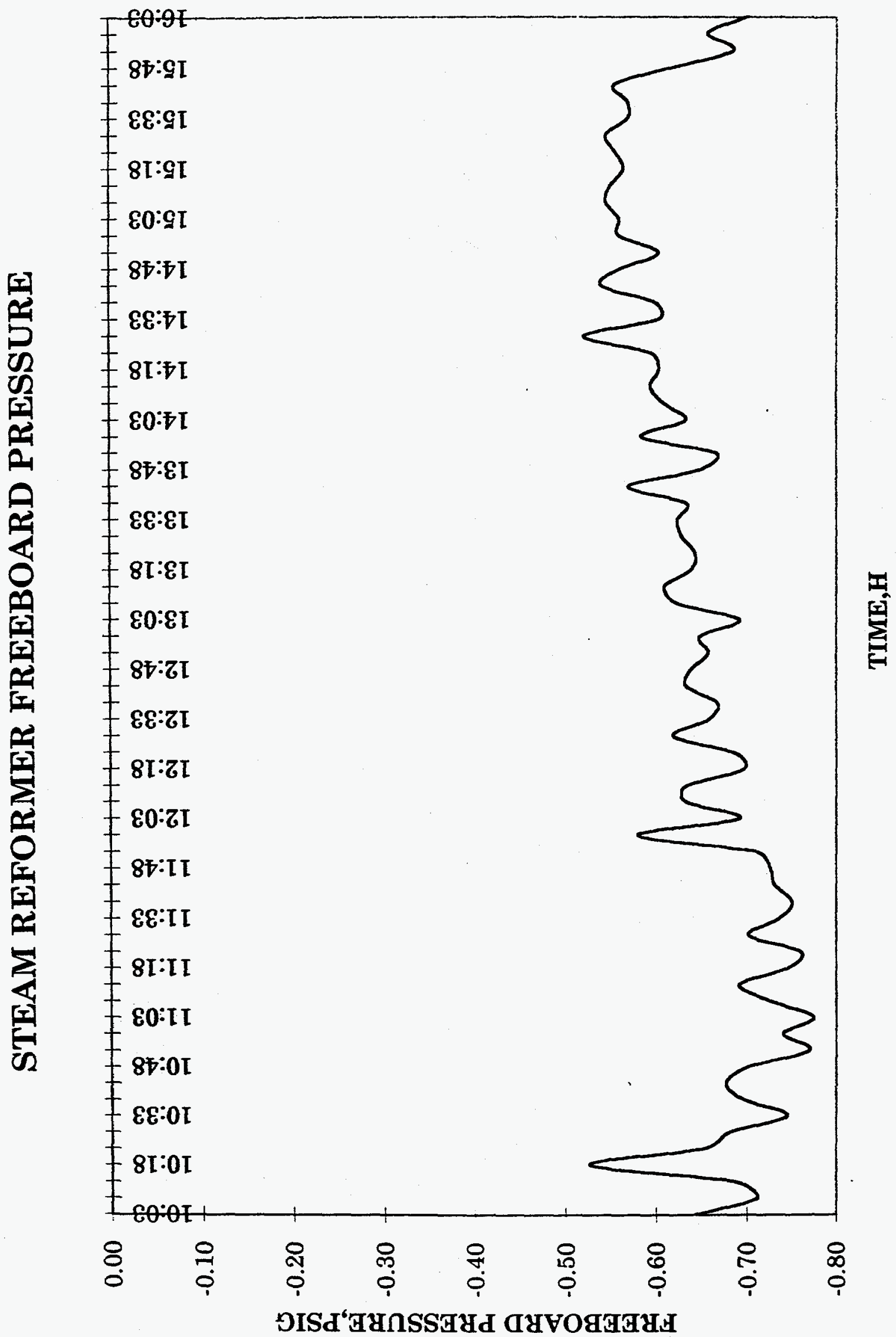




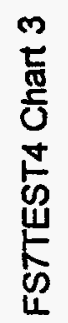
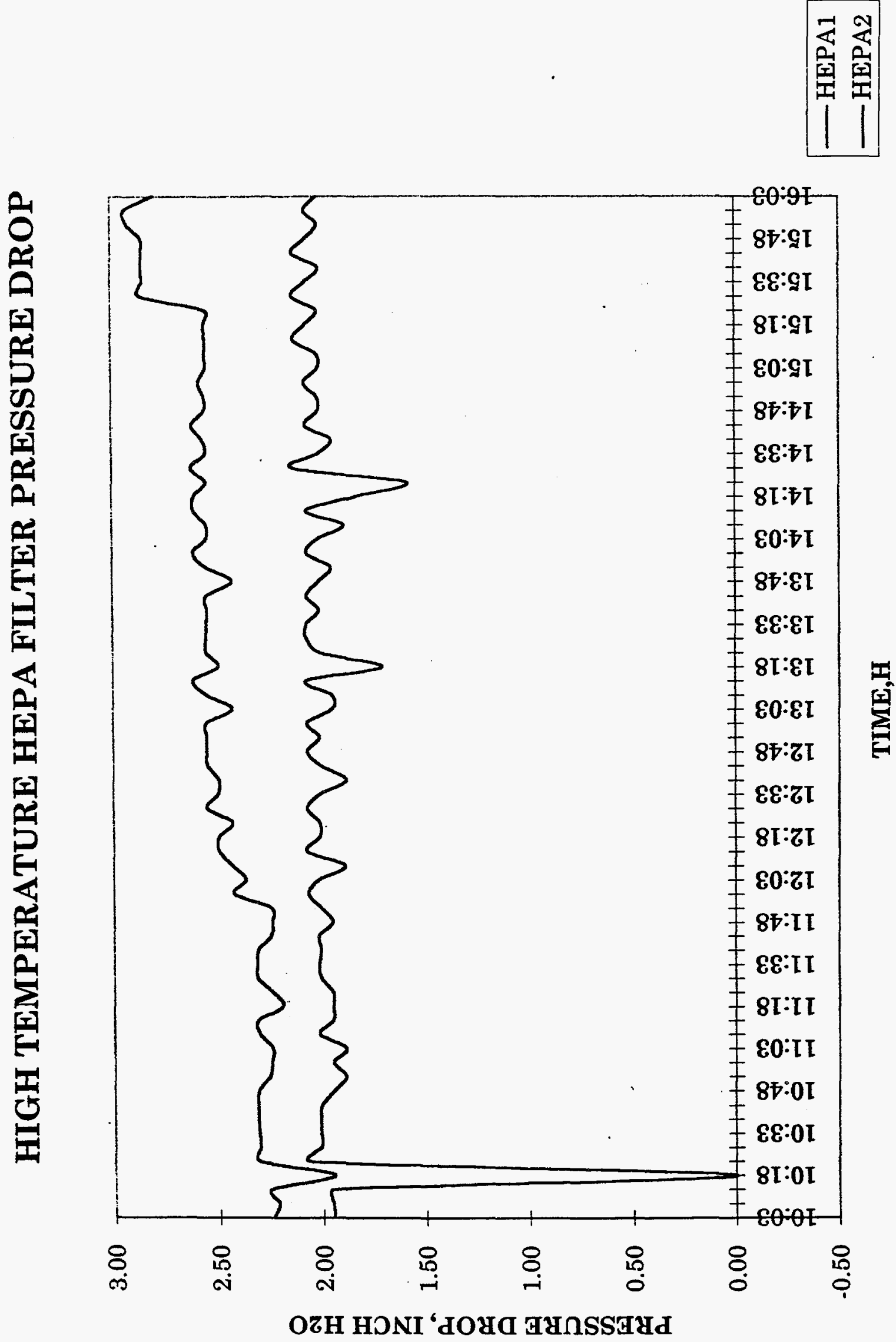


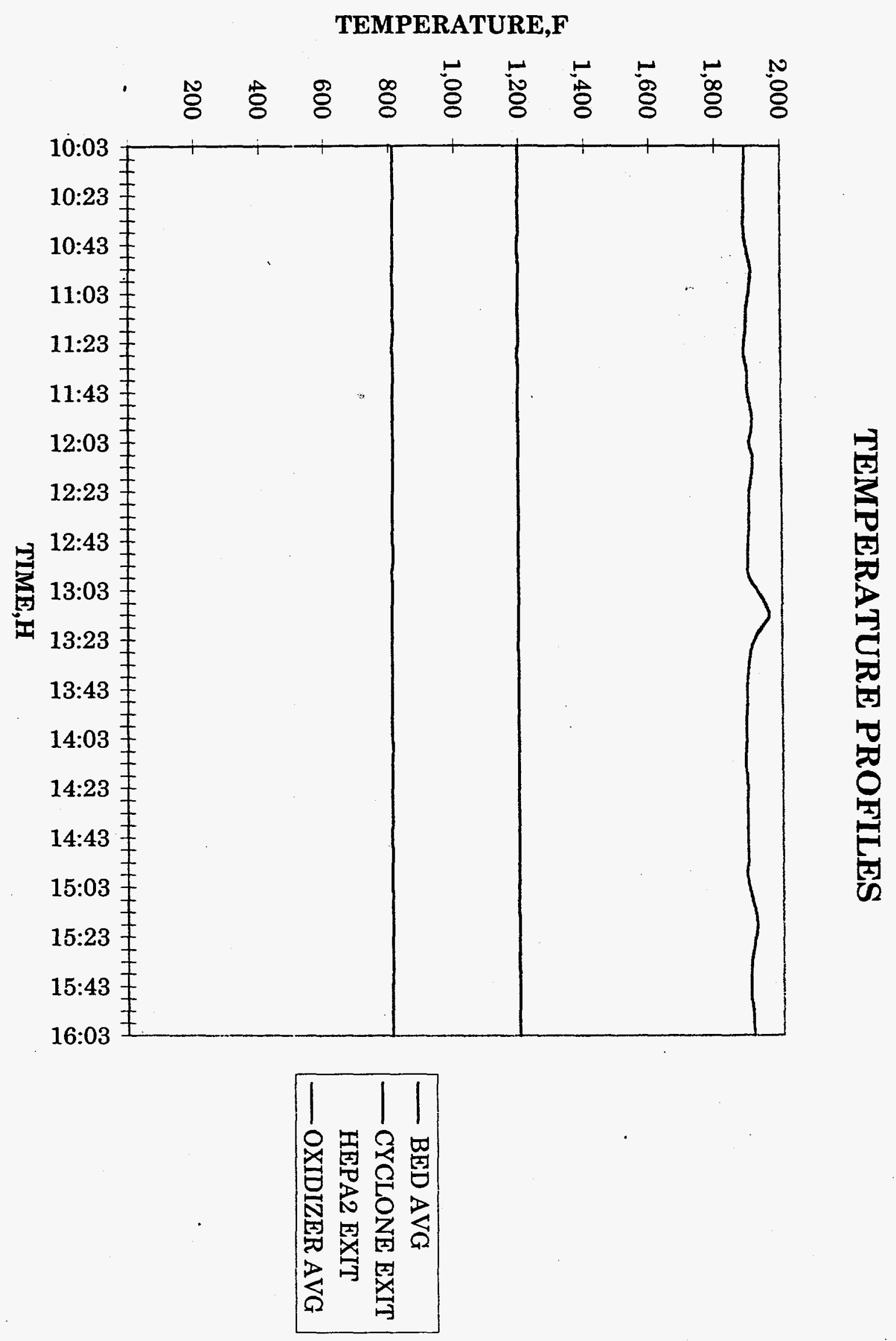




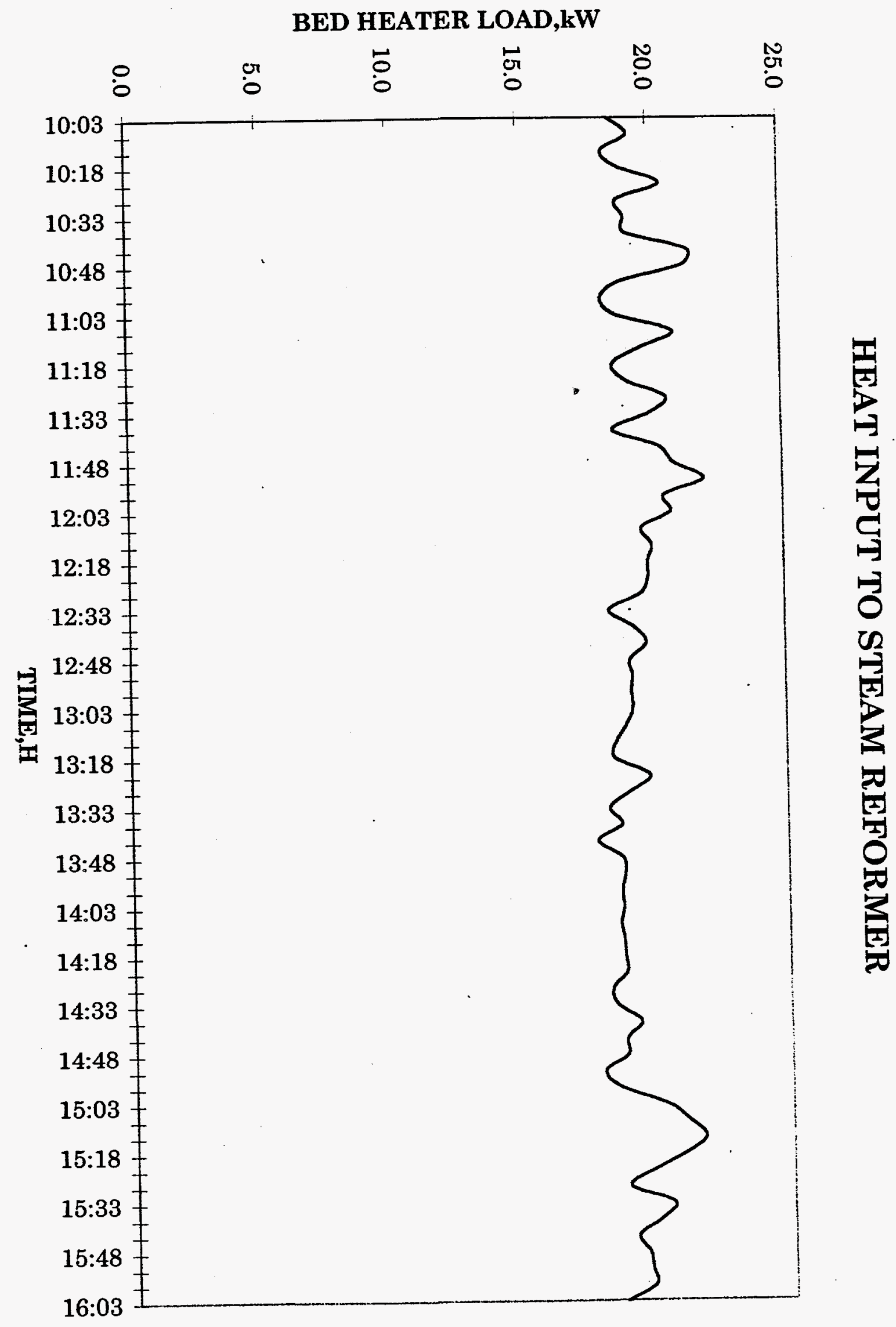




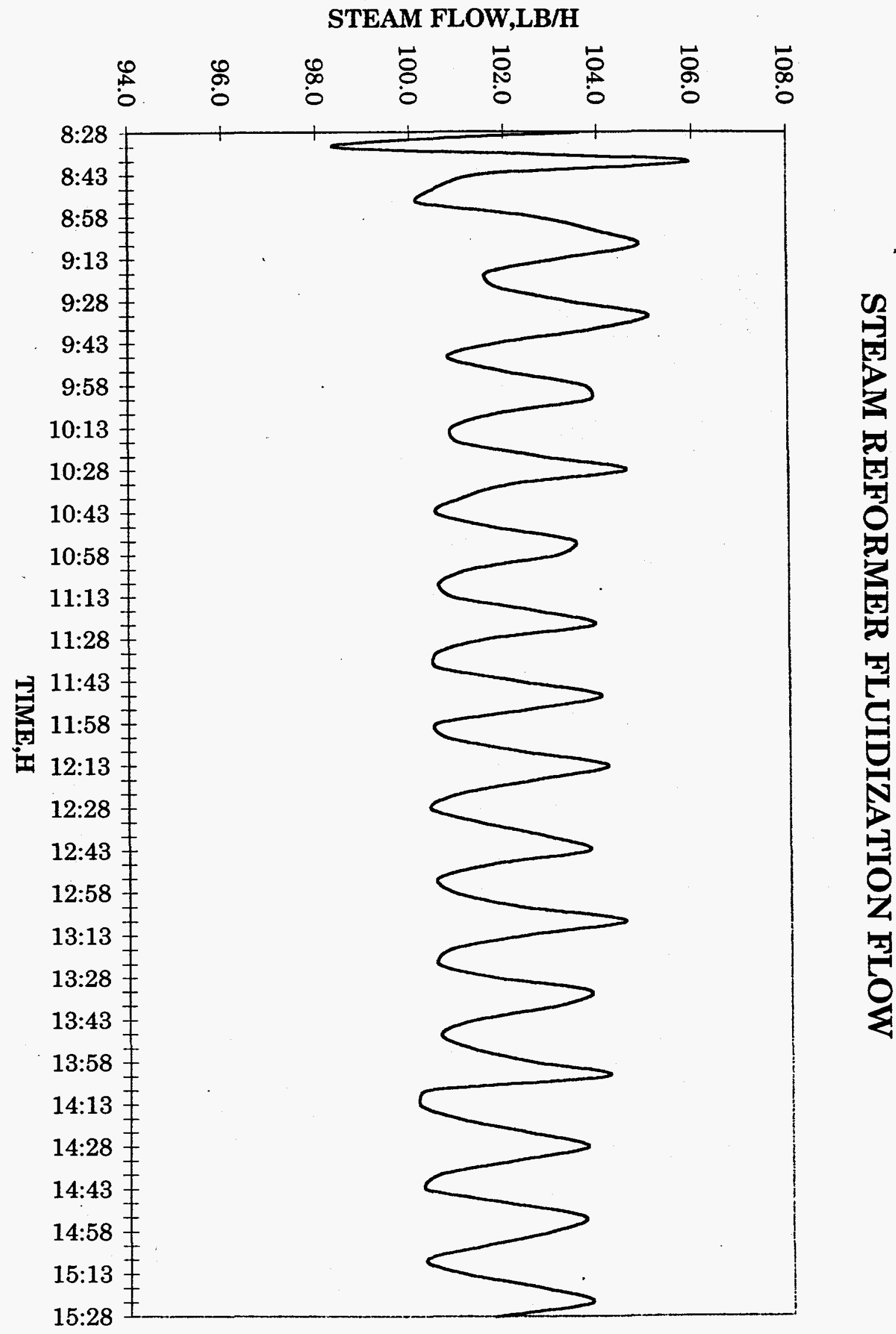

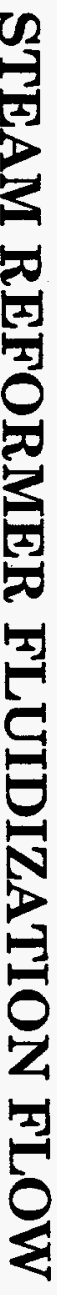




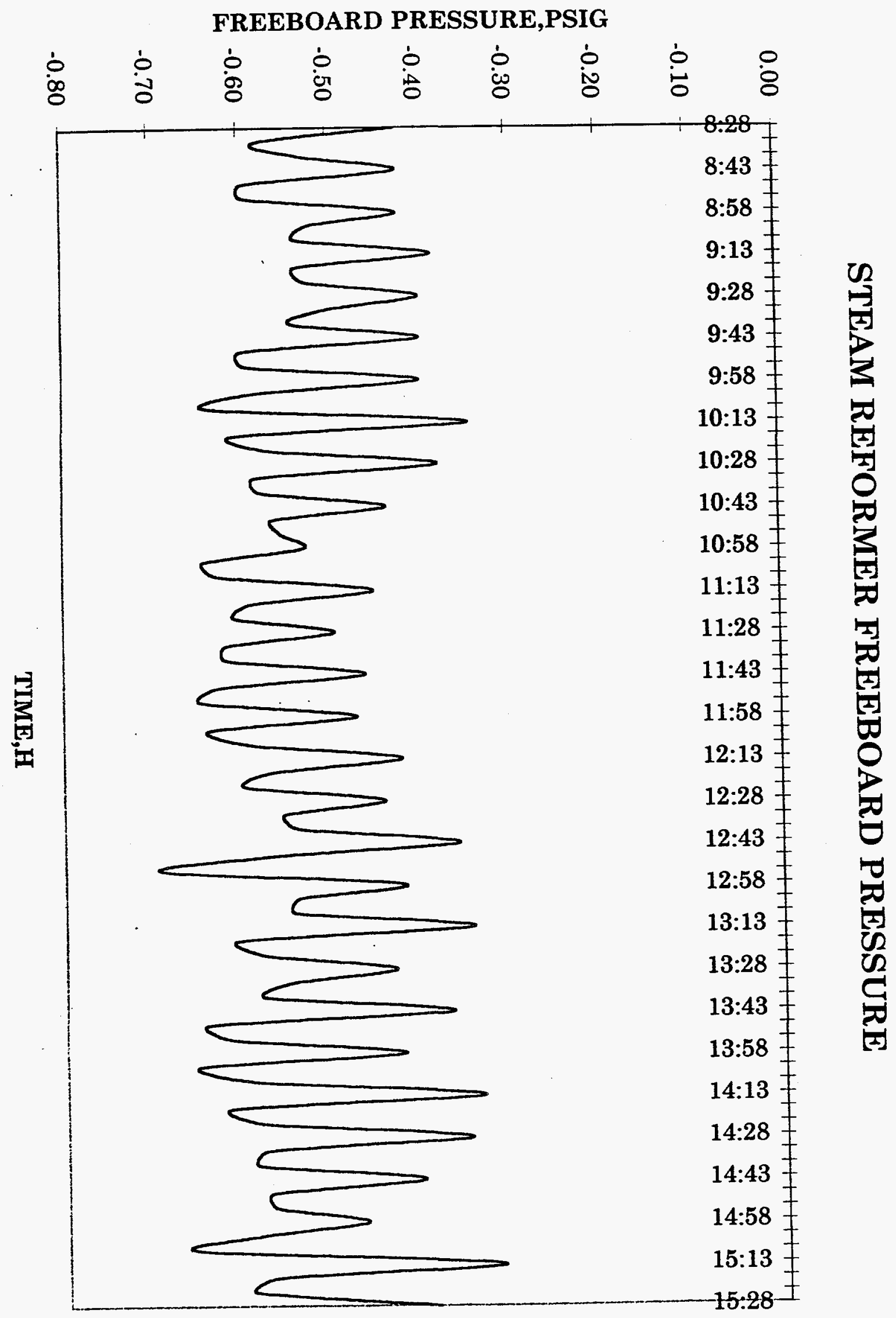




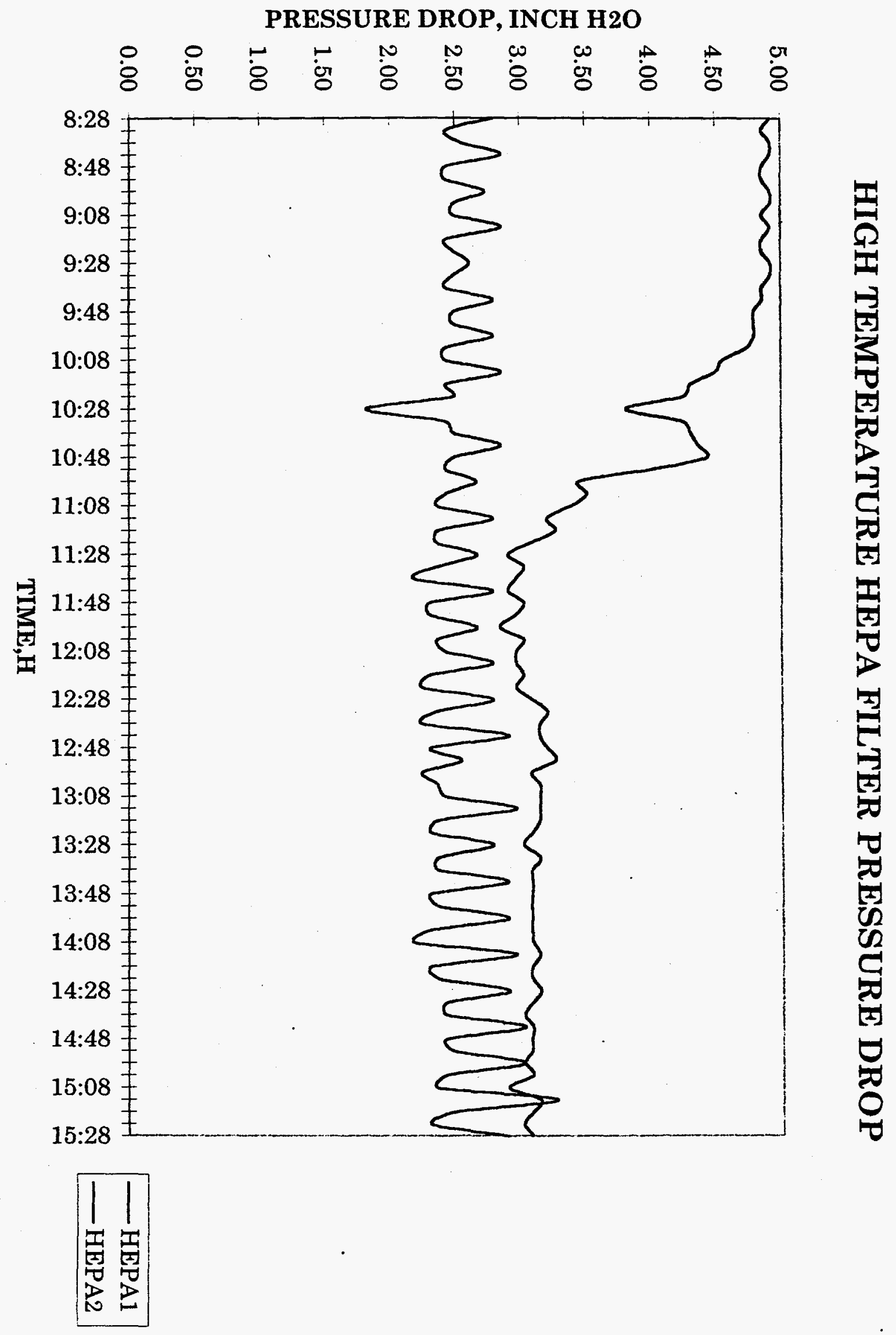




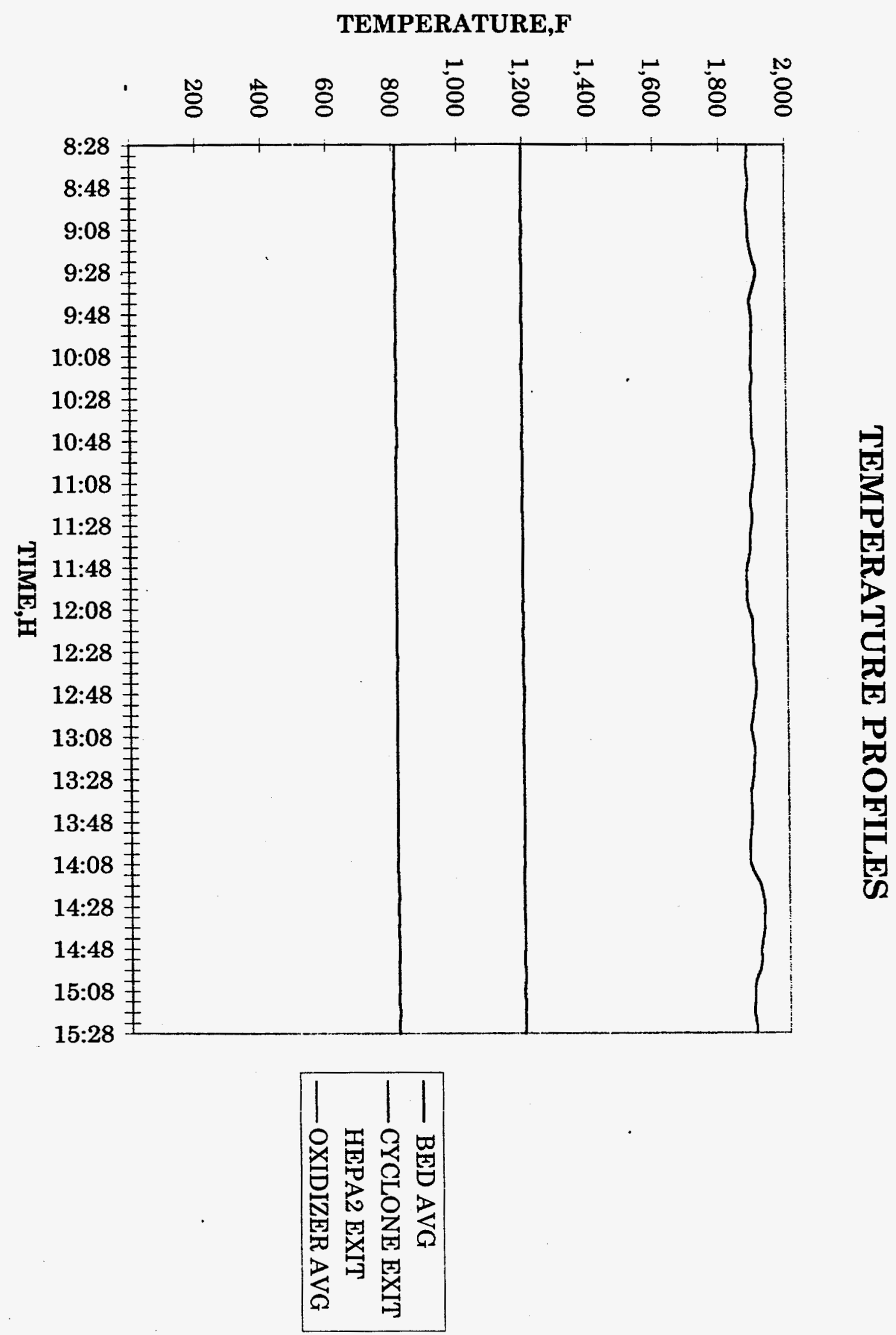




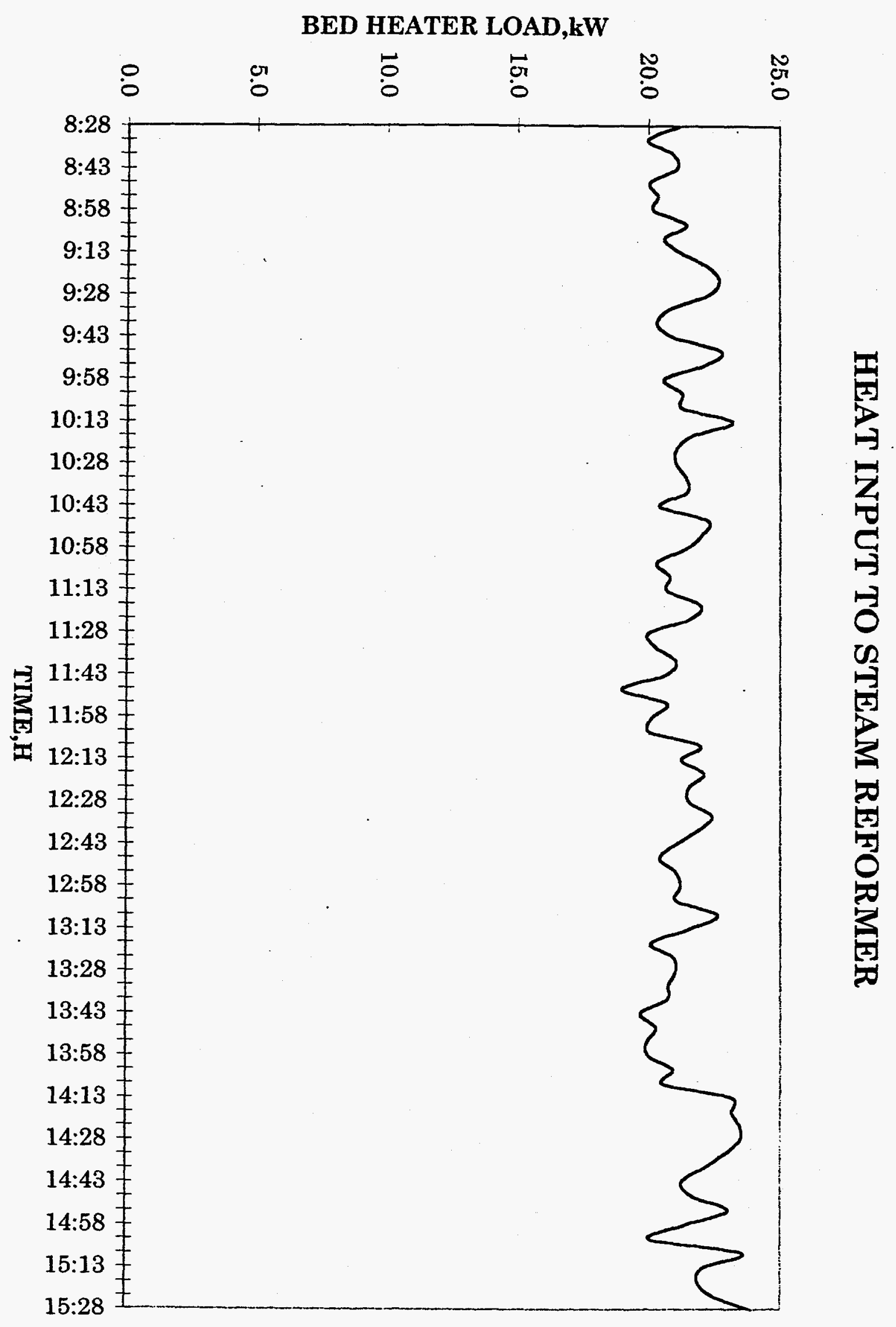




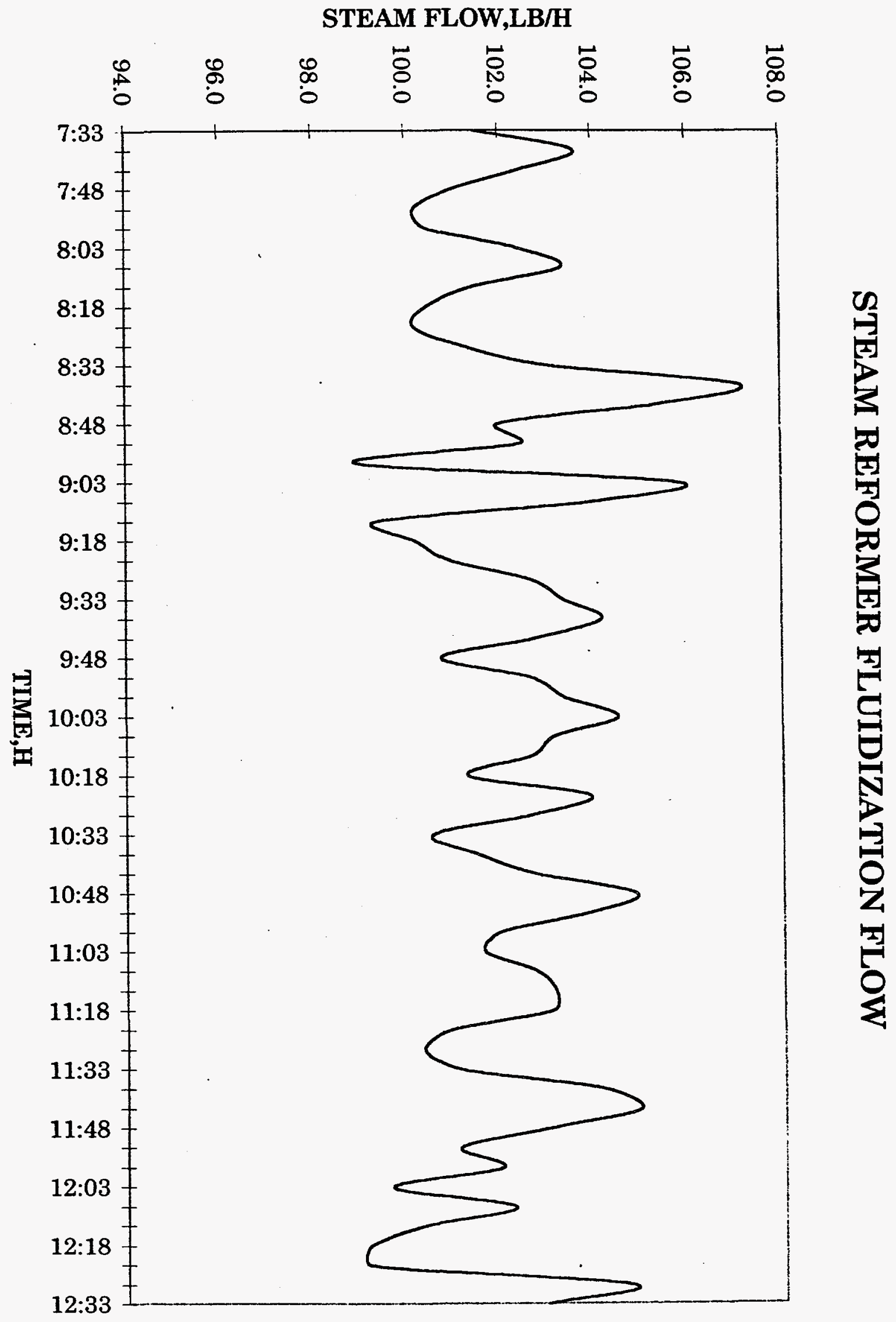


FREEBOARD PRESSURE,PSIG

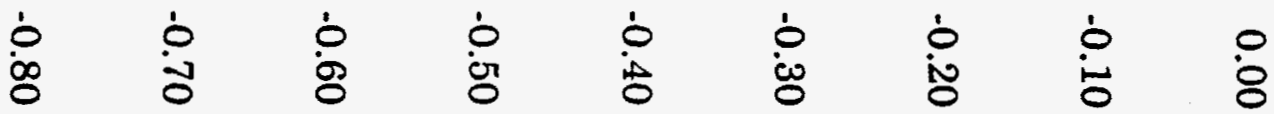

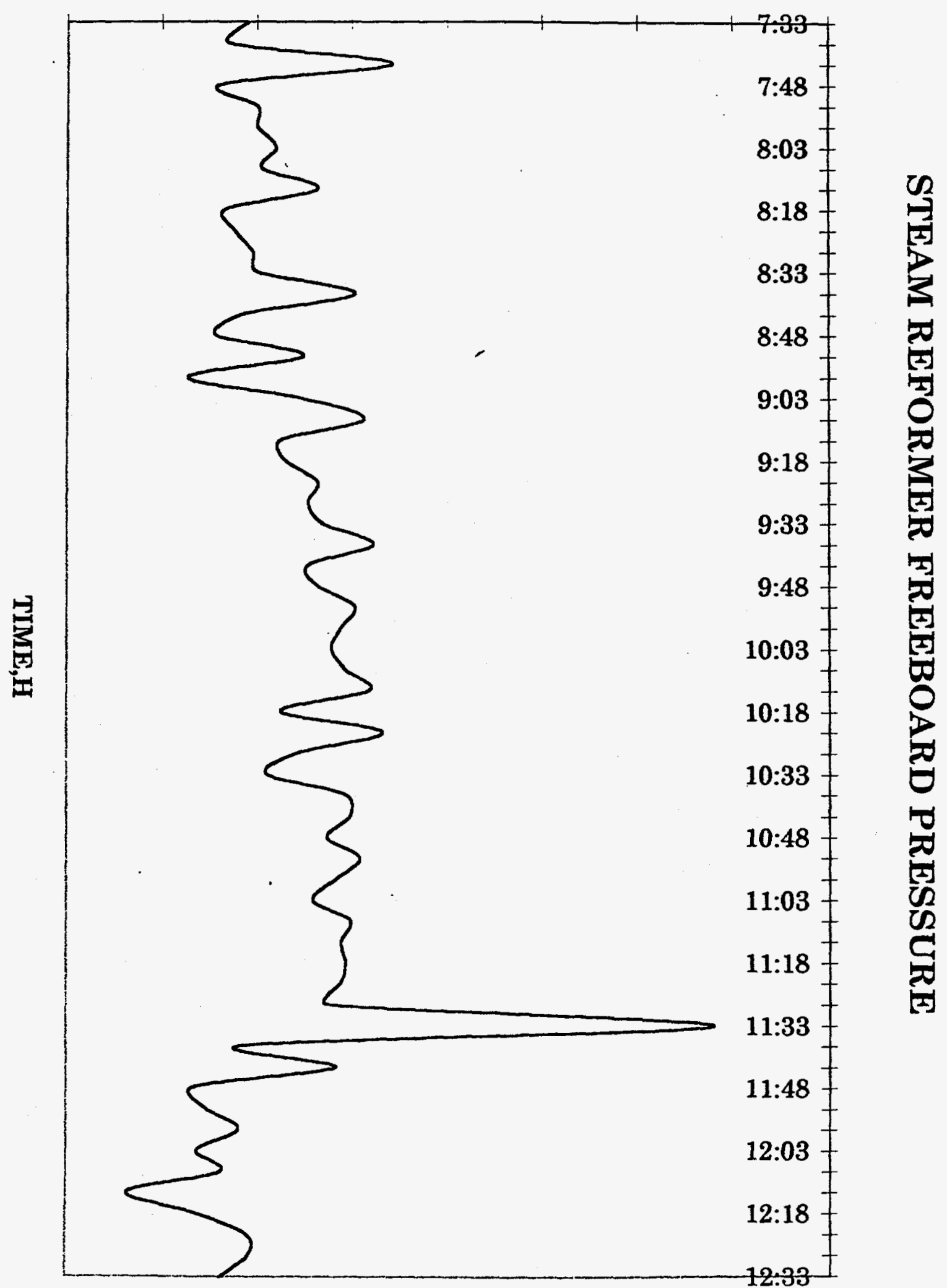




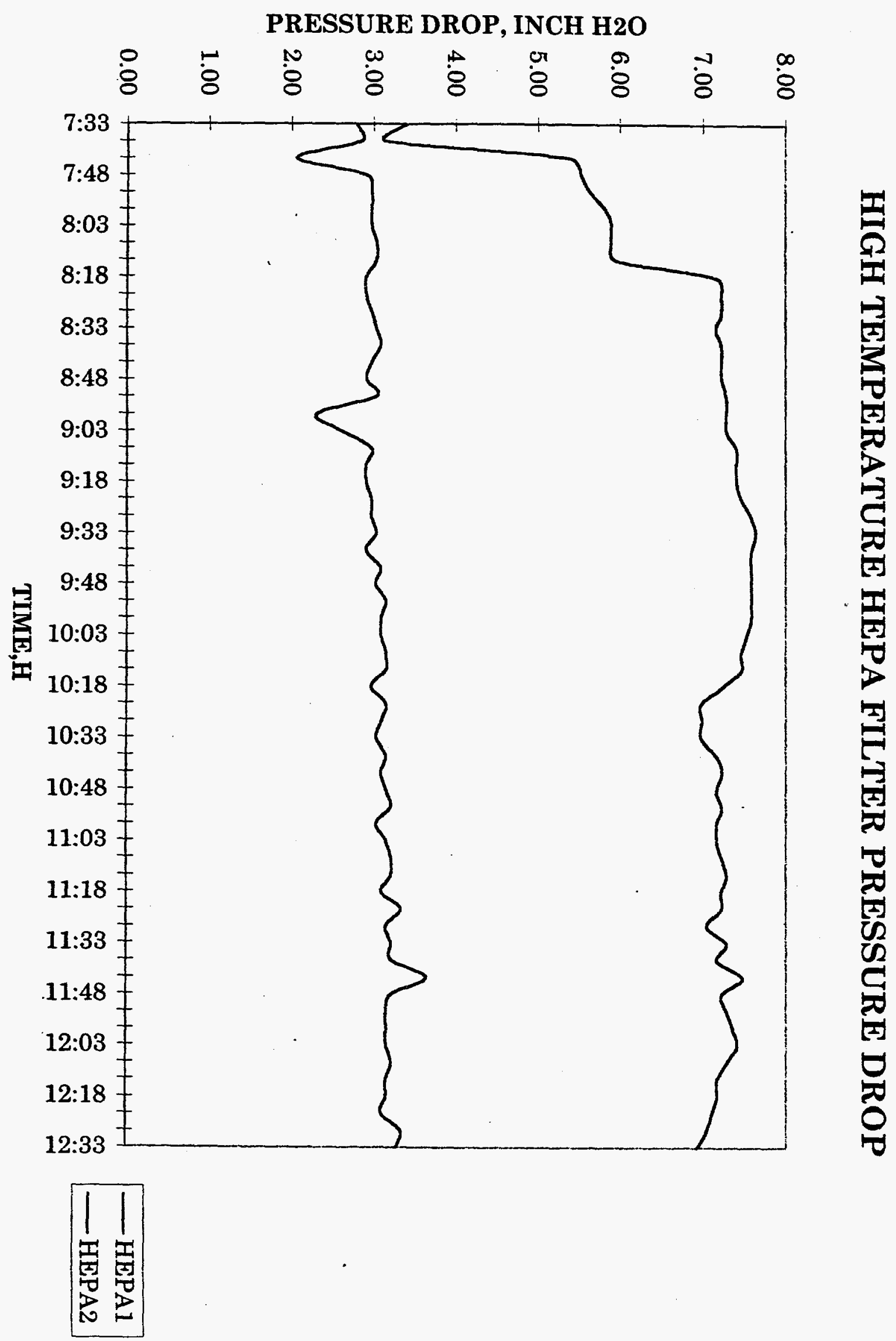




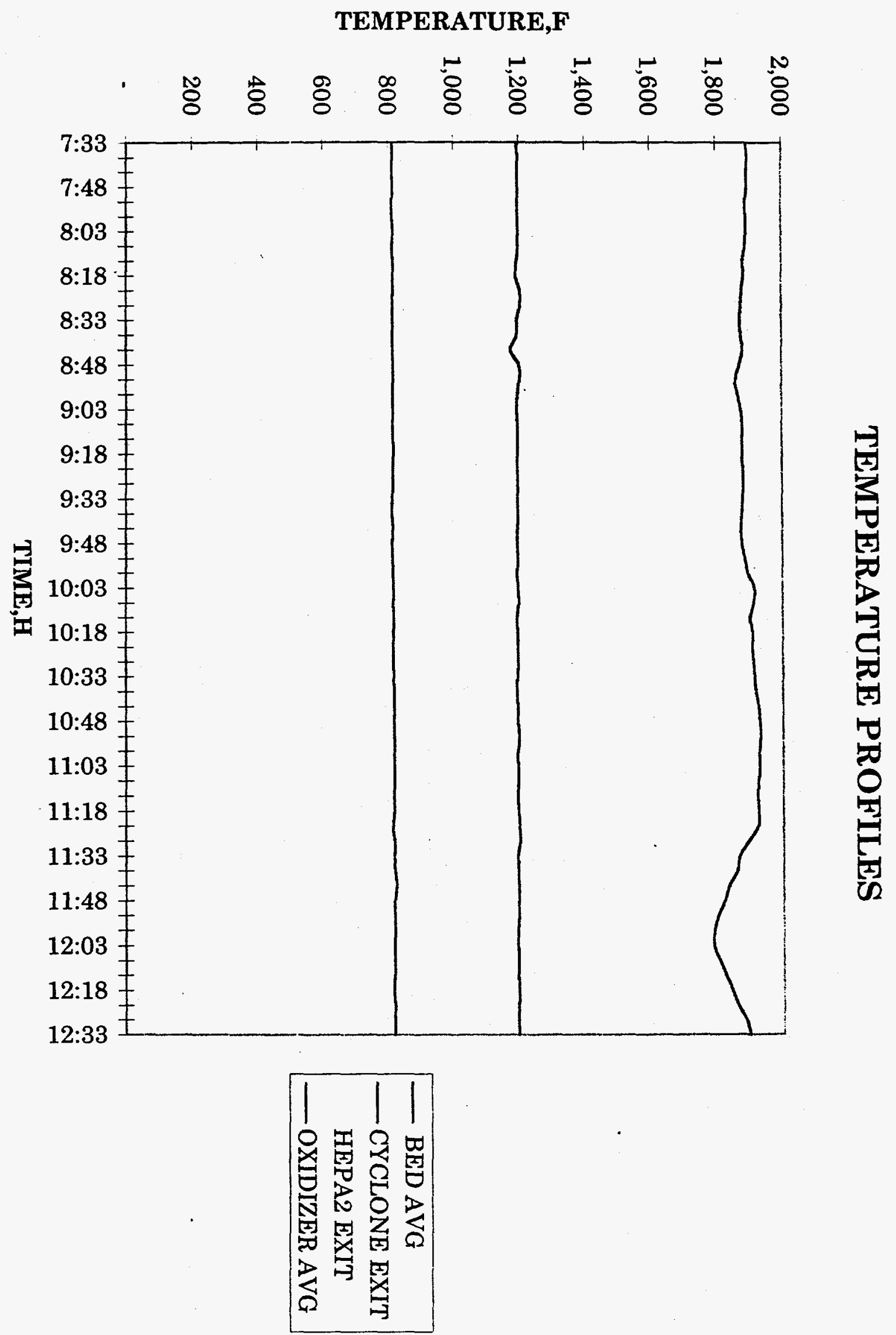




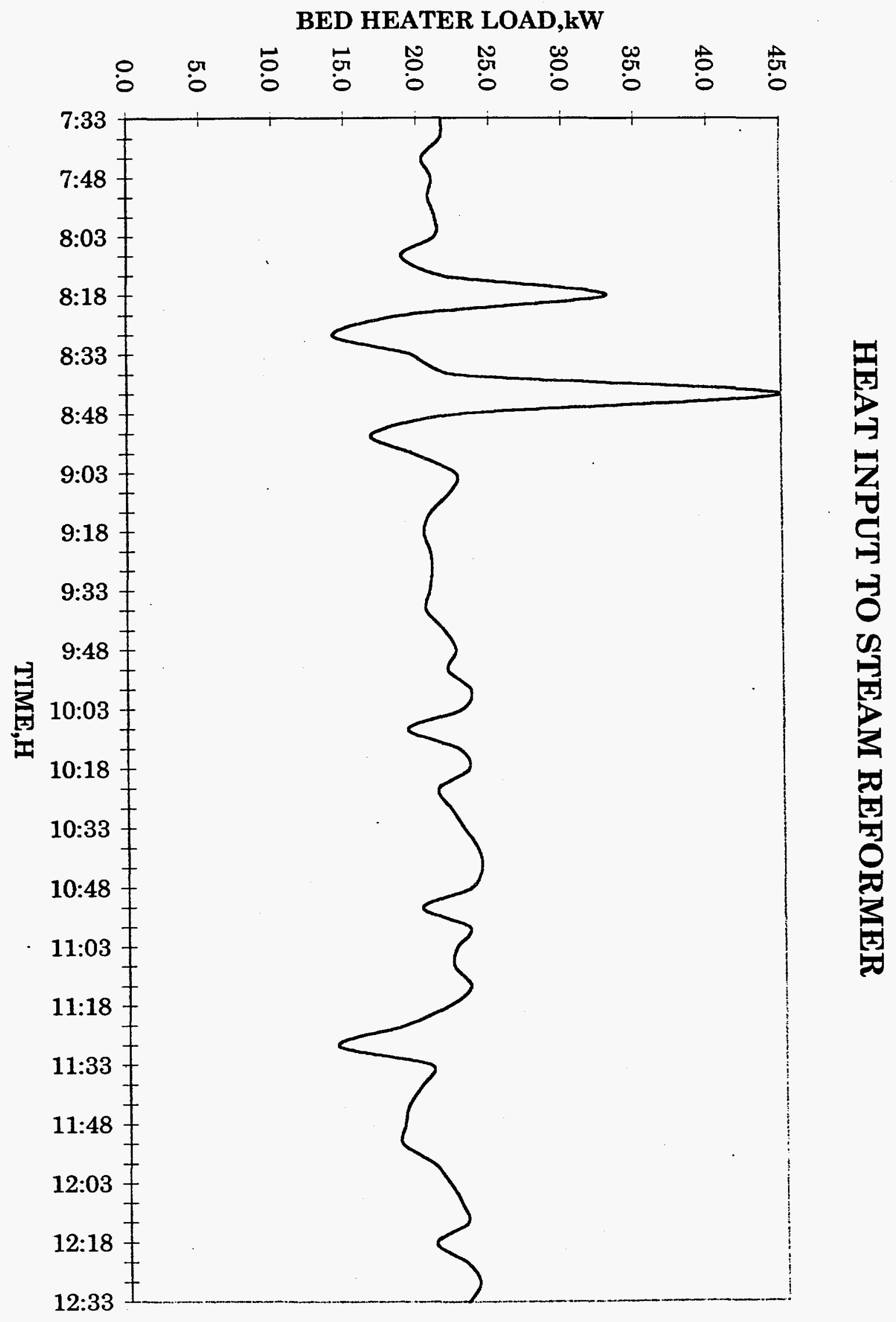

